\title{
EXISTENCE AND A PRIORI ESTIMATES FOR EUCLIDEAN GIBBS STATES
}

\author{
S. ALBEVERIO, YU. KONDRATIEV, T. PASUREK, AND M. RÖCKNER
}

\begin{abstract}
We prove a priori estimates and, as a sequel, the existence of Euclidean Gibbs states for quantum lattice systems. For this purpose we develop a new analytical approach, the main tools of which are: first, a characterization of the Gibbs states in terms of their Radon-Nikodým derivatives under shift transformations as well as in terms of their logarithmic derivatives through integration by parts formulae, and second, the choice of appropriate Lyapunov functionals describing stabilization effects in the system. The latter technique becomes applicable since on the basis of the integration by parts formulae the Gibbs states are characterized as solutions of an infinite system of partial differential equations. Our existence results generalize essentially all previous ones. In particular, superquadratic growth of the interaction potentials is allowed and $N$-particle interactions for $N \in \mathbb{N} \cup\{\infty\}$ are included. We also develop abstract frames both for the necessary single spin space analysis and for the lattice analysis apart from their applications to our concrete models. Both types of general results obtained in these two frames should also be of their own interest in infinite-dimensional analysis.
\end{abstract}

\section{INTRODUCTION}

I. Background, methods and purpose. This paper is concerned with models of quantum anharmonic lattice systems. In statistical physics they are commonly viewed as models for quantum crystals and are closely related to (Euclidean) quantum lattice field theory with continuous time (cf. [DLP79, GJ81]). A mathematical description of equilibrium properties of quantum systems might be given in terms of their Gibbs states. However, a corresponding general concept of quantum Gibbs states (including their rigorous definition) such as that based on the standard algebraic approach [BrRo81] still remains open. Thus we shall take the Euclidean approach. This approach is conceptually analogous to the well-known Euclidean strategy in quantum field theory (see e.g. Sim74, GJ81. This analogy was pointed out and first developed in AH-K75, (see also the recent developments in AKKR01, AKKR02). It transforms the problem of constructing quantum Gibbs states as functionals on the algebra of local observables into the problem of studying certain Euclidean Gibbs measures $\mu$ on the loop lattice $\Omega_{\beta}:=\left[C\left(S_{\beta}\right)\right]^{\mathbb{Z}^{d}}$. Here $\beta>0$ has the meaning of the inverse temperature and $S_{\beta} \cong[0, \beta]$ is a circle with Lebesgue measure $d \tau$. For a more detailed discussion of the relations between quantum and Euclidean Gibbs states we refer to the Appendix (Sect. 8) below.

As compared with classical lattice systems, the situation with Euclidean Gibbs measures of quantum anharmonic systems is much more rich and complicated, since for

2000 Mathematics Subject Classification. Primary 82B10; Secondary 46G12, 60H30.

Key words and phrases. Quantum lattice systems; Euclidean Gibbs states; smooth measures on infinite-dimensional spaces and their logarithmic derivatives; integration by parts formulae; Lyapunov functionals. 
the latter systems the single spin spaces (e.g., $C\left(S_{\beta}\right)$ or $L_{\beta}^{R}\left(S_{\beta}\right), R \geq 1$ ) themselves are infinite dimensional and their topological features should be properly taken into account.

On the other hand, Gibbs measures, usually defined by local specifications through the Dobrushin-Lanford-Ruelle equations (see, e.g., [Pr76, Ge88]) can also be described via their Radon-Nikodym derivatives under shift transformations of the configuration spaces (cf. Theorem 4.6 in AKR97b] and its extension in Proposition 4.2 below). If the interaction potentials are smooth enough, this flow characterization is in turn equivalent to the characterization of Gibbs states as differentiable measures satisfying integration by parts formulas with prescribed logarithmic derivatives (cf. Proposition 4.9). As is typical for noncompact spin spaces, we have to restrict ourselves to the set $\mathcal{G}_{t}$ of tempered Gibbs measures $\mu$, which we specify by some natural support condition (see Definition 3.9). The aim of this paper is to show that such alternative descriptions in a direct analytical way give both the existence of Euclidean Gibbs measures $\mu \in \mathcal{G}_{t}$ (cf. Main Theorem I in Subsect. 2.3) and a priori estimates on their correlations in terms of parameters of the interaction (cf. Main Theorem II in Subsect. 2.3).

The essential ingredient of the proofs is that the characterization of Gibbs measures via integration by parts gives the possibility of dealing with them as with the solutions of an infinite system of first-order PDE's

$$
\partial_{h_{i}} \mu=b_{i} \cdot \mu, \quad i \in \mathbb{N} .
$$

Here the $h_{i}$ are some admissible directions forming an orthonormal basis in the tangent Hilbert space $\mathcal{H}:=l^{2}\left(\mathbb{Z}^{d}\right) \otimes L^{2}\left(S_{\beta}\right)$ and the $b_{i}$ are the corresponding partial logarithmic derivatives of (all) measures $\mu \in \mathcal{G}_{t}$ along $h_{i}$. This enables us to employ the Lyapunov function method (in a similar way as in finite-dimensional PDE's) in order to get a priori moment estimates on $\mu \in \mathcal{G}^{t}$. However, when dealing with the integration by parts formulas (1.1), the main difficulty one encounters is that one does not know in advance whether $b_{i} \in L^{1}(\mu)$ for any fixed direction $h_{i}$ and measure $\mu \in \mathcal{G}_{t}$. This problem is successfully overcome by a special choice of test functions $f$ to which we can correctly apply both sides of the distributional identity (1.1). The local Gibbs specifications also satisfy the same integration by parts formulas, from which we deduce moment estimates uniformly in volume. The latter is crucial for our proof of the existence result for Euclidean Gibbs measures $\mu$, i.e., $\mathcal{G}_{t} \neq \emptyset$.

It should be mentioned that this approach was first realized for classical lattice systems in AKRT99, AKRT00. However, the abstract scheme, in the form suggested in those papers, is not directly applicable (see also BR01, BRW01]) to the present case. The reason is that for Euclidean Gibbs states we have to perform not only "lattice analysis", but need also a separate and quite different "single spin space analysis".

Some results on the existence of Euclidean Gibbs measures, concerning specific classes of anharmonic interactions and using various techniques, have been known before (see Subsection 2.6 for the references and a detailed discussion of the previous "state of the art" of the problem). But we emphasize that our technique is completely different and rather elementary, provided one has the integration by parts description of Gibbs measures (or uses it as a definition). We also would like to point out an advantage of our approach over the so-called stochastic dynamics method, since now we can avoid the extremely difficult ergodicity problems for the related infinite-dimensional stochastic evolution equations (cf. the corresponding discussion in Subsect. 5.3). We would also like to stress that our approach for the first time gives the existence of Euclidean Gibbs states for many-particle interactions with infinite radius and superquadratic growth, which has not been possible to obtain by other known methods. In addition, we obtain useful and precise information on the support properties of all tempered Euclidean Gibbs states. 
By reason of notational simplicity only, in this paper we decided to restrict ourselves to the systems of one-dimensional (i.e., polarized) oscillators. However, all the results obtained naturally extend to the multi-dimensional case. A possible way for obtaining such a generalization is described in [AKPR04.

II. Structure and contents of the paper. Along with the Introduction (Section 1) and the Appendix (Section 8), the paper consists of two parts, namely, Part I: "Gibbs setting" (Sections 2-5) and Part II: "Abstract setting" (Sections 6, 7).

Part $\mathbf{I}$ is devoted to general aspects of the theory of Euclidean Gibbs measures and their characterization through integration by parts formulas.

Section 2: In order to present the main ideas, we start with some particular classes of quantum lattice systems, "anharmonic crystals". Then we formulate our main Theorems I-III on the existence and a priori estimates for tempered Euclidean Gibbs measures and compare them with the previous results obtained by other methods.

Section 3: We introduce the general models of quantum lattice systems and discuss the assumptions on the interactions (which are not necessarily translation invariant and are possibly of infinite range). Next, we describe in detail the corresponding Gibbsian formalism and define the set $\mathcal{G}_{t}$ of all tempered Euclidean Gibbs measures $\mu$ on the loop lattice $\Omega$.

Section 4: We begin with the alternative description of $\mu \in \mathcal{G}_{t}$ in terms of its RadonNikodým derivatives under shifts on the configuration space (see Proposition 4.1). Then we prove an equivalent description of $\mu \in \mathcal{G}_{t}$ as differentiable measures satisfying (in the distributional sense by pairing with proper test functions $f$ ) the integration by parts formula (1.1) with the given logarithmic derivatives $b_{i}$ (see Proposition 4.9).

Section 5: Here we demonstrate the applications of the (IbP)-formula to the study of $\mu \in \mathcal{G}_{t}$. Also we discuss the validity of the same flow and integration by parts characterizations for local Gibbs specifications. Finally, we give elementary proofs of our Main Theorems I-III, but under some Hypotheses $(\mathbf{H})$ and $\left(\mathbf{H}_{l o c}\right)$, which are proved to always hold for our systems subsequently in Section 7 .

In Part II we transfer the problem in a general framework of symmetrizing measures on Banach lattices.

Section 6: Here we perform the "single spin space analysis" on a general Banach space $X$, which later on will be taken as the loop space $C\left(S_{\beta}\right)$. More precisely, we study symmetrizing measures $\mu \in \mathcal{M}^{b}(X)$ on a single Banach space $X$ (i.e., those measures satisfying integration by parts formulas with some prescribed partial logarithmic derivatives $b_{i}$ ). We suppose that the logarithmic derivatives have a linear component $A$ (being a positive selfadjoint operator in some tangent Hilbert space $H \supseteq X)$ and the nonlinear one $F$ (possessing certain coercivity properties w.r.t. $H$ ). Developing a Lyapunov functional method in this situation, we derive a priori estimates on the moments of $\mu \in \mathcal{M}^{b}(X)$.

Section 7: We further enrich the abstract setting of the previous section by adding an extra "lattice structure". So, in order to include the case of Euclidean Gibbs measures, we consider symmetrizing measures $\mu \in \mathcal{M}^{b}(\mathcal{X})$ on Banach lattices $\mathcal{X}:=X^{\mathbb{Z}^{d}}$. The required a priori estimates on $\mu \in \mathcal{M}^{b}(\mathcal{X})$ are formulated as Theorems 7.1 and 7.3. At the end of this section we come back to the Euclidean Gibbs measures and (on the basis of Theorems 7.1 and 7.3 just proved) verify the validity of the Hypotheses $(\mathbf{H})$ and $\left(\mathbf{H}_{1 \text { oc }}\right)$ for them.

In the Appendix (Section 8) we enclose a discussion on the Euclidean approach to quantum Gibbs states.

Finally we mention that the results of the paper have been announced in AKPR01a, AKPR01b, AKPR04 and presented in various talks since December 2000 during seminars or conferences, e.g., in Berlin, Kiev, Moscow, Oberwolfach, and Pisa. 
The authors express their gratitude to Yuri Kotitsky and Robert Minlos for many stimulating discussions, and a special thank to Oles Kutovyi and Dmitrii Finkelstein for the assistance in preparing a Russian variant of the manuscript.

\section{PART I: GibBs SETting}

\section{Particular models of QUANTUM ANHARMONiC CRYSTAls}

In order to fix the main ideas and make the reader more familiar with the topic, in this section we give a self-contained presentation of some particular models of quantum lattice systems ( $Q L S$, for shorthand). So, in Subsects. 2.1-2.3 we concentrate on the simplest case of the translation invariant system with the harmonic pair interaction between nearest neighbors only (cf. Model I). Following the Euclidean approach, in Subsect. 2.2 we define the corresponding quantum Gibbs states as classical Gibbs measures, but with infinite-dimensional single spin (i.e., loop) spaces. In Subsect. 2.3 we formulate our main results on the existence and a priori estimates for tempered Gibbs measures on loop lattices. Further modifications that one needs in order to treat the case of the pair interaction potentials of superquadratic growth (Model II), resp. of possibly infinite range (Model III), are briefly described in Subsect. 2.4, resp. 2.5. Finally, in Subsect. 2.6 we discuss fundamental problems and basic methods in the study of Euclidean Gibbs states, as well as compare our results with those previously obtained by other authors.

2.1. Model I: Harmonic pair interaction. We start with the following simplest and sufficiently popular in the literature model of a quantum crystal. Let $\mathbb{Z}^{d}\left(\subset \mathbb{R}^{d}\right)$ be the $d$-dimensional integer lattice with the Euclidean distance $|k-j|, k, j \in \mathbb{Z}^{d}$. We consider an infinite system of interacting quantum particles performing one-dimensional (i.e., polarized) oscillations with displacements $q_{k} \in \mathbb{R}$ around their equilibrium positions at points $k \in \mathbb{Z}^{d}$. Each particle individually is described by the quantum mechanical Hamiltonian

$$
\mathbb{H}_{k}:=-\frac{1}{2 \mathfrak{m}} \frac{d^{2}}{d q_{k}^{2}}+\frac{a^{2}}{2} q_{k}^{2}+V\left(q_{k}\right)
$$

acting in the (physical) Hilbert state space $\mathcal{H}_{k}:=L^{2}\left(\mathbb{R}, d q_{k}\right)$. Here $\mathfrak{m}\left(=\mathfrak{m}_{p h} / \hbar^{2}\right)>0$ is the (reduced) mass of the particles and $a^{2}>0$ is their rigidity w.r.t. harmonic oscillations. Concerning the anharmonic self-interaction potential, we suppose that $V \in C^{2}(\mathbb{R} \rightarrow \mathbb{R})$, i.e., twice continuously differentiable, and, moreover, that it satisfies the following growth condition:

Assumption $\left(\mathbf{V}_{\mathbf{0}}\right)$. There exist some constants $P>2$ and $K_{V}, C_{V}>0$ such that for all $q \in \mathbb{R}$,

$$
K_{V}^{-1}|q|^{P-l}-C_{V} \leq(\operatorname{sgn} q)^{l} \cdot V^{(l)}(q) \leq K_{V}|q|^{P-l}+C_{V}, \quad l=0,1,2 .
$$

Next, we add the harmonic nearest-neighbor interaction $W\left(q_{k}, q_{k^{\prime}}\right):=J\left(q_{k}-q_{k^{\prime}}\right)^{2}$ with the intensity $J>0$, the sum being taken over all (unordered) pairs $\left\langle k, k^{\prime}\right\rangle$ in $\mathbb{Z}^{d}$ such that $\left|k-k^{\prime}\right|=1$. The whole system is then described by a heuristic Hamiltonian of the form

$$
\mathbb{H}:=-\frac{1}{2 \mathfrak{m}} \sum_{k \in \mathbb{Z}^{d}} \frac{d^{2}}{d q_{k}^{2}}+\frac{a^{2}}{2} \sum_{k \in \mathbb{Z}^{d}} q_{k}^{2}+\sum_{k \in \mathbb{Z}^{d}} V\left(q_{k}\right)+J \sum_{\left\langle k, k^{\prime}\right\rangle \subset \mathbb{Z}^{d}}\left(q_{k}-q_{k^{\prime}}\right)^{2} .
$$

Actually, the infinite-volume Hamiltonian (2.2) cannot be defined directly as a mathematical object and is represented by the local (i.e., indexed by finite volumes $\Lambda \subset \mathbb{Z}^{d}$ ) 
Hamiltonians

$$
\mathbb{H}_{\Lambda}:=\sum_{k \in \Lambda} H_{k}+J \sum_{\left\langle k, k^{\prime}\right\rangle \subset \Lambda}\left(q_{k}-q_{k^{\prime}}\right)^{2}
$$

(as selfadjoint and lower bounded Schrödinger operators) acting in the Hilbert spaces $\mathcal{H}_{\Lambda}:=\bigotimes_{k \in \Lambda} \mathcal{H}_{k}$

Lattice systems of the above type (as well as their generalizations studied in Sect. 3 below) are commonly viewed in quantum statistical physics as mathematical models of a crystalline substance (for more physical background see e.g. [DLP79, FM99, MVZ00, AKKR02). The study of such systems is especially motivated by the reason that they provide a mathematically rigorous and physically quite realistic description for the important phenomenon of phase transitions (i.e., nonuniqueness of Gibbs states). So, if the potential $V$ has a double-well shape, in the large mass limit $\mathfrak{m} \rightarrow \infty$, then the QLS (2.2) may undergo (ferroelectic) structural phase transitions connected with the appearance of macroscopic displacements of particles for low temperatures $\beta^{-1}<\beta_{c r}^{-1}$ ( $\mathfrak{m}$ ) (for a mathematical justification of this effect see e.g. [BaK91, HM00]).

Remark 2.1. (i) In fact, from the potential $V$ one can always extract the quadratic term $a^{2} q^{2} / 2$ with a small $a^{2}>0$, so that $\left(\mathbf{V}_{\mathbf{0}}\right)$ is still true. Typical potentials satisfying Assumption $\left(\mathbf{V}_{\mathbf{0}}\right)$ are polynomials of even degree and with a positive leading coefficient, i.e.,

$$
P(q):=V(q):=\sum_{1 \leq l \leq 2 n} b_{l} q^{l} \text { with } b_{2 n}>0 \text { and } n \geq 2 .
$$

In this case one speaks about so-called ferromagnetic $P(\phi)$-models, which also can naturally be looked upon as lattice discretizations of quantum $P(\phi)$-fields (cf. Sim74, GJ81]). Let us also mention a special choice in (2.3), when

$$
P(q):=\sum_{0 \leq l \leq n} b_{2 l} q^{2 l} \text { with } b_{2 l} \geq 0 \text { for all } 2 \leq l \leq n .
$$

Since $b_{2} \in \mathbb{R}$ can be a large negative number, such polynomials may have arbitrary deep double wells. Moreover, the last systems are technically more suitable for the study of critical behaviour and the influence of quantum effects, since then one can use not only the FKG correlation inequalities, which are standard for ferromagnetic pair interactions, but also more advanced (e.g., GKS, Lebowitz) inequalities relying on the additional symmetry properties of the one-particle potential (2.4) (cf. AKKR02 as well as a discussion in Subsect. 2.6 below).

(ii) All constructions in Subsects. 2.1-2.3 remain true if one takes for $W\left(q_{k}, q_{k^{\prime}}\right)$ the general ferromagnetic interaction $U\left(q_{k}-q_{k^{\prime}}\right)$ given by a nonnegative convex function $U \in C^{2}(\mathbb{R} \rightarrow \mathbb{R})$ satisfying

$$
0 \leq \inf _{\mathbb{R}} U^{\prime \prime} \leq \sup _{\mathbb{R}} U^{\prime \prime}<\infty, \quad 0 \leq U(q)=U(-q), \forall q \in \mathbb{R} .
$$

Notation 2.2. Throughout the paper we shall use the following notation. For a set $\Lambda \subset \mathbb{Z}^{d}$, we denote by $|\Lambda|$ its cardinality, by diam $\Lambda:=\sup _{k, k^{\prime} \in \Lambda}\left|k-k^{\prime}\right|$ its diameter, by $\Lambda^{c}:=\mathbb{Z}^{d} \backslash \Lambda$ its complement, and by $\partial \Lambda:=\left\{k^{\prime} \in \Lambda^{c}|\exists k \in \Lambda| k-,k^{\prime} \mid=1\right\}$ its boundary. In particular, $\partial k:=\left\{k^{\prime} \in \mathbb{Z}^{d}|| k-k^{\prime} \mid=1\right\}$ is the set of all neighbors of $k$ consisting of $2 d$ points. We write $\Lambda \Subset \mathbb{Z}^{d}$ whenever $1 \leq|\Lambda|<\infty$. As usual, $\Lambda \nearrow \mathbb{Z}^{d}$ means the limit as $N \rightarrow \infty$ along any increasing sequence of volumes $\Lambda^{(N)} \subset \Lambda^{(N+1)} \Subset \mathbb{Z}^{d}$ such that $\bigcup_{N \in \mathbb{N}} \Lambda^{(N)}=\mathbb{Z}^{d}$. 
2.2. Definition of Euclidean Gibbs measures. As was already mentioned in the Introduction, we take the Euclidean approach based on a path space representation for quantum Gibbs states. Since such an approach involves intricate relations between quantum statistical mechanics and stochastic processes, for convenience of the nonexpert reader we enclose a more extended discussion of the related topics in the Appendix. Now we briefly describe the corresponding Euclidean Gibbsian formalism just for the concrete class of quantum lattice systems (2.2); for all necessary details presented already in the context of the general model (3.1) we refer the reader to Section 3 below.

Let $S_{\beta} \cong[0, \beta]$ be a circle of length $\beta$, where we fix positive $0<\beta:=(k T)^{-1}$ having the meaning of inverse temperature. For each $k \in \mathbb{Z}^{d}$, denote by

$$
\begin{gathered}
C_{\beta}^{n+\alpha}:=C^{n+\alpha}\left(S_{\beta} \rightarrow \mathbb{R}\right), \quad n \in \mathbb{N} \cup\{0\}, \alpha \in(0,1), \\
L_{\beta}^{r}:=L^{r}\left(S_{\beta} \rightarrow \mathbb{R}, d \tau\right), \quad r \geq 1,
\end{gathered}
$$

the standard Banach spaces of all (Hölder) continuous, resp. integrable (w.r.t. Lebesgue measure $d \tau$ ), functions, i.e., loops, $\omega_{k}: S_{\beta} \rightarrow \mathbb{R}$. In particular, $C_{\beta}$ with the sup-norm $|\cdot|_{C_{\beta}}$ will be treated as the single spin space, whereas $L_{\beta}^{2}$ with the inner product $(\cdot, \cdot)_{L_{\beta}^{2}}:=|\cdot|_{L_{\beta}^{2}}^{2}$ will be treated as the Hilbert space tangent to $C_{\beta}$.

As the configuration space for the infinite volume system we define the product loop space

$$
\Omega:=\left[C_{\beta}\right]^{\mathbb{Z}^{d}}=\left\{\omega=\left(\omega_{k}\right)_{k \in \mathbb{Z}^{d}} \mid \omega: S_{\beta} \rightarrow \mathbb{R}^{\mathbb{Z}^{d}}, \omega_{k} \in C_{\beta}\right\}
$$

equipped with the corresponding Borel $\sigma$-algebra $\mathcal{B}(\Omega)$. Let $\mathcal{M}(\Omega)$ denote the set of all probability measures on $(\Omega, \mathcal{B}(\Omega)$ ). Next, we introduce the subset of ("exponentially increasing") tempered configurations

$$
\Omega_{(e) t}:=\left\{\omega \in \Omega \mid \forall \delta \in(0,1):\|\omega\|_{-\delta}:=\left[\sum_{k \in \mathbb{Z}^{d}} e^{-\delta|k|}\left|\omega_{k}\right|_{L_{\beta}^{2}}^{2}\right]^{\frac{1}{2}}<\infty\right\}
$$

and respectively the subset of tempered measures supported by $\Omega_{(e) t} \in \mathcal{B}(\Omega)$, i.e.,

$$
\mathcal{M}_{(e) t}:=\left\{\mu \in \mathcal{M}(\Omega) \mid \mu\left(\Omega_{(e) t}\right)=1\right\} .
$$

Heuristically, the Euclidean Gibbs measures $\mu$ we are interested in have the following representation:

$$
d \mu(\omega):=Z^{-1} \exp \{-\mathcal{I}(\omega)\} \prod_{k \in \mathbb{Z}^{d}} d \gamma_{\beta}\left(\omega_{k}\right),
$$

where $Z$ is the normalization factor, $\gamma_{\beta}$ is a centered Gaussian measure on $\left(C_{\beta}, \mathcal{B}\left(C_{\beta}\right)\right)$ with correlation operator $A_{\beta}^{-1}$, and $A_{\beta}:=-\mathfrak{m} \Delta_{\beta}+a^{2} \mathbf{1}$ is the shifted Laplace-Beltrami operator on the circle $S_{\beta}$. Respectively the map

$$
\Omega \ni \omega \longmapsto \mathcal{I}(\omega):=\int_{S_{\beta}}\left[\sum_{k \in \mathbb{Z}^{d}} V\left(\omega_{k}\right)+\sum_{\left\langle k, k^{\prime}\right\rangle \subset \mathbb{Z}^{d}} W\left(\omega_{k}, \omega_{k^{\prime}}\right)\right] d \tau
$$

might be viewed as a potential energy functional describing an interacting system of loops $\omega_{k} \in C_{\beta}$ indexed by $k \in \mathbb{Z}^{d}$. Of course it is impossible to use this presentation for $\mu$ literally, since the series in (2.9) do not converge in any sense. We recall that, relying on the Dobrushin-Lanford-Ruelle (DLR) formalism (cf. [Do70, Pr76, Ge88), a rigorous meaning can be given to the measures $\mu$ as random fields on $\mathbb{Z}^{d}$ with a prescribed family of local specifications $\left\{\pi_{\Lambda}\right\}_{\Lambda \Subset \mathbb{Z}^{d}}$ in the following way: 
For $\Lambda \Subset \mathbb{Z}^{d}, \pi_{\Lambda}$ is defined as a probability kernel on $(\Omega, \mathcal{B}(\Omega))$ : for all $\Delta \in \mathcal{B}(\Omega)$ and $\xi \in \Omega$,

$$
\pi_{\Lambda}(\Delta \mid \xi):=Z_{\Lambda}^{-1}(\xi) \int_{\Omega_{\Lambda}} \exp \left\{-\mathcal{I}_{\Lambda}(\omega \mid \xi)\right\} \mathbf{1}_{\Delta}\left(\omega_{\Lambda}, \xi_{\Lambda^{c}}\right) \prod_{k \in \Lambda} d \gamma_{\beta}\left(\omega_{k}\right)
$$

(where $\mathbf{1}_{\Delta}$ denotes the indicator on $\Delta$ ). Here $Z_{\Lambda}(\xi)$ is the normalization factor and

$$
\mathcal{I}_{\Lambda}(\omega \mid \xi):=\int_{S_{\beta}}\left[\sum_{k \in \Lambda} V\left(\omega_{k}\right)+\sum_{\left\langle k, k^{\prime}\right\rangle \subset \Lambda} W\left(\omega_{k}, \omega_{k^{\prime}}\right)+\sum_{k \in \Lambda, k^{\prime} \in \Lambda^{c}} W\left(\omega_{k}, \xi_{k^{\prime}}\right)\right] d \tau
$$

is the interaction in the volume $\Lambda$ under the boundary condition $\xi_{\Lambda^{c}}:=\left(\xi_{k^{\prime}}\right)_{k^{\prime} \in \Lambda^{c}}$. Obviously, $\inf _{\omega \in \Omega} \mathcal{I}_{\Lambda}(\omega \mid \xi)>-\infty$ and the RHS in (2.11) makes sense for the potentials $V, W$ we deal with here. An important point is the consistency property for $\left\{\pi_{\Lambda}\right\}_{\Lambda \Subset \mathbb{Z}^{d}}$ : for all $\Lambda \subset \Lambda^{\prime} \Subset \mathbb{Z}^{d}, \xi \in \Omega$ and $\Delta \in \mathcal{B}(\Omega)$,

$$
\left(\pi_{\Lambda^{\prime}} \pi_{\Lambda}\right)(\Delta \mid \xi):=\int_{\Omega} \pi_{\Lambda^{\prime}}(d \omega \mid \xi) \pi_{\Lambda}(\Delta \mid \omega)=\pi_{\Lambda^{\prime}}(\Delta \mid \xi)
$$

Definition 2.3. A probability measure $\mu$ on $(\Omega, \mathcal{B}(\Omega))$ is called a Euclidean Gibbs measure for the specification $\left\{\pi_{\Lambda}\right\}_{\Lambda \Subset \mathbb{Z}^{d}}$ (corresponding to the quantum lattice system (2.2) at inverse temperature $\beta>0)$ if it satisfies the DLR equilibrium equations: for all $\Lambda \Subset \mathbb{Z}^{d}$ and $\Delta \in \mathcal{B}(\Omega)$,

$$
\mu \pi_{\Lambda}(\Delta):=\int_{\Omega} \mu(d \omega) \pi_{\Lambda}(\Delta \mid \omega)=\mu(\Delta) .
$$

Fixing $\beta>0$, let $\mathcal{G}$ denote the set of all such measures $\mu$. We shall be concerned with the subset $\mathcal{G}_{(e) t}$ of tempered Gibbs measures supported by $\Omega_{(e) t}$, i.e.,

$$
\mathcal{G}_{(e) t}:=\mathcal{G} \cap \mathcal{M}_{(e) t}=\left\{\mu \in \mathcal{G} \mid \mu\left(\Omega_{(e) t}\right)=1\right\} .
$$

Remark 2.4. (i) Later it will be instructive to compare our results on quantum systems with the analogous classical ones. The large-mass limit $\mathfrak{m} \rightarrow \infty$ (or $\hbar \rightarrow 0$ ) of model (2.2) gives us an infinite system of interacting classical particles moving in the external field $V$. Such a system is described by the potential energy functional

$$
H_{c l}(q)=\frac{a^{2}}{2} \sum_{k \in \mathbb{Z}^{d}} q_{k}^{2}+\sum_{k \in \mathbb{Z}^{d}} V\left(q_{k}\right)+\sum_{\left\langle k, k^{\prime}\right\rangle \subset \mathbb{Z}^{d}} W\left(q_{k}, q_{k^{\prime}}\right)
$$

on the configuration space $\Omega_{c l}:=\mathbb{R}^{\mathbb{Z}^{d}} \ni\left\{q_{k}\right\}_{k \in \mathbb{Z}^{d}}:=q$ (see AKKR02]). Again, the formal Hamiltonian (2.14) does not make sense itself and is represented by the local Hamiltonians

$$
\mathbb{H}_{c l, \Lambda}(q \mid y):=\frac{a^{2}}{2} \sum_{k \in \Lambda} q_{k}^{2}+\sum_{k \in \Lambda} V\left(q_{k}\right)+\sum_{\left\langle k, k^{\prime}\right\rangle \subset \Lambda} W\left(q_{k}, q_{k^{\prime}}\right)+\sum_{k \in \Lambda, k^{\prime} \in \Lambda^{c}} W\left(q_{k}, y_{k^{\prime}}\right)
$$

in the volumes $\Lambda \Subset \mathbb{Z}^{d}$ given the boundary conditions $y \in \Omega_{c l}$. The corresponding Gibbs states $\mu \in \mathcal{G}_{c l}$ at inverse temperature $\beta>0$ are defined as probability measures on $\Omega_{c l}$ satisfying the DLR equations $\mu \pi_{\Lambda}=\mu, \Lambda \Subset \mathbb{Z}^{d}$, with the family of local specifications (2.16)

$$
\pi_{\Lambda}(\Delta \mid y):=Z_{\Lambda}^{-1}(y) \int_{\mathbb{R}^{\Lambda}} \exp \left\{-\beta H_{c l, \Lambda}(q \mid y)\right\} \mathbf{1}_{\Delta}\left(q_{\Lambda}, y_{\Lambda^{c}}\right) \prod_{k \in \Lambda} d q_{k}, \quad \Delta \in \mathcal{B}\left(\Omega_{c l}\right), \quad y \in \Omega_{c l} .
$$

Starting from the pioneering papers [LP76, Ro77, COPP78, BH-K82, such unbounded spin systems have been under intensive investigation in classical statistical mechanics (for recent developments see, e.g., [AKRT00, BH00, Yo01]). 
(ii) Our definition of temperedness (as well as its modification to the classical systems (2.14) with $\left|q_{k}\right|$ instead of $\left|\omega_{k}\right|_{L_{\beta}^{2}}$ ) is less restrictive (and simpler) than those usually used in the literature (for comparison, see e.g. [COPP78, BH-K82]). So, obviously, $\Omega_{(e) t} \supseteq \Omega_{(s) t}$, resp. $\mathcal{M}_{(e) t} \supseteq \mathcal{M}_{(s) t}$, where the subsets of all ( slowly increasing") tempered configurations, resp. measures, are defined by

$$
\begin{aligned}
\Omega_{(s) t}:= & \left\{\omega \in \Omega \mid \exists p=p(\omega)>0:\|\omega\|_{-p}:=\left[\sum_{k \in \mathbb{Z}^{d}}(1+|k|)^{-2 p}\left|\omega_{k}\right|_{L_{\beta}^{2}}^{2}\right]^{\frac{1}{2}}<\infty\right\}, \\
& \mathcal{M}_{(s) t}:=\left\{\mu \in \mathcal{M}(\Omega) \mid \exists p=p(\mu)>0:\|\omega\|_{-p}<\infty \text { for } \mu \text {-a.e. } \omega \in \Omega\right\} .
\end{aligned}
$$

Moreover, $\mathcal{M}_{(s) t}$ contains all measures $\mu \in \mathcal{M}(\Omega)$ satisfying the following condition in terms of their moment sequence:

$$
\exists p=p(\mu)>0: \quad \sum_{k \in \mathbb{Z}^{d}}(1+|k|)^{-2 p} E_{\mu}\left|\omega_{k}\right|_{L_{\beta}^{2}}^{2}<\infty,
$$

in particular, those having the so-called Ruelle support (see Remark 5.11 (ii) below). Here and further on we write

$$
E_{\mu} f:=\int f d \mu
$$

for any $\mu$-integrable function $f$.

(iii) If it does not lead to the reader's confusion, as a rule in the concrete model setting we shall just use the standard notation $\Omega_{t}, \mathcal{M}_{t}$ and $\mathcal{G}_{t}$ by omitting all the additional suband superscripts (as, e.g., here $(e)$ or $(s)$ ).

2.3. Formulation of the main results. Now we are ready to present our results on the existence and a priori estimates for Euclidean Gibbs measures corresponding to the QLS (2.2). We assume that all the conditions on the interaction potentials imposed in Subsect.2.1 are fulfilled without mentioning them again in the formulations of our statements.

Main Theorem I (Existence of Tempered Gibbs States). For all values of the mass $\mathfrak{m}>0$ and the inverse temperature $\beta>0$ :

$$
\mathcal{G}_{t} \neq \emptyset .
$$

Main Theorem II (A Priori Estimates on Tempered Gibbs States). Every $\mu \in \mathcal{G}_{t}$ is supported by the set of Hölder loops $\bigcap_{0 \leq \alpha<\frac{1}{2}}\left[C_{\beta}^{\alpha}\right]^{\mathbb{Z}^{d}}$. Moreover, for all $Q \geq 1$ and $\alpha \in\left[0, \frac{1}{2}\right)$,

$$
\sup _{\mu \in \mathcal{G}_{t}} \sup _{k \in \mathbb{Z}^{d}} \int_{\Omega}\left|\omega_{k}\right|_{C_{\beta}^{\alpha}}^{Q} d \mu(\omega)<\infty .
$$

Corollary from Theorem II. The set $\mathcal{G}_{t}$ is compact w.r.t. the topology of weak convergence of measures on any of the spaces $\left[C_{\beta}^{\alpha}\right]^{\mathbb{Z}^{d}}, 0 \leq \alpha<1 / 2$, equipped with the system of seminorms $\left|\omega_{k}\right|_{C_{\beta}^{\alpha}}, k \in \mathbb{Z}^{d}$.

Actually, the existence result for $\mu \in \mathcal{G}_{t}$ immediately follows by Prokhorov's tightness criterion from the moment estimate (2.19) for the family $\pi_{\Lambda}(d \omega \mid \xi=0)$, which holds uniformly in volume $\Lambda \Subset \mathbb{Z}^{d}$. Moreover, the a priori estimates for the probability kernels $\pi_{\Lambda}(d \omega \mid \xi)$ of the local specification, subject to the fixed boundary condition $\xi \in \Omega_{t}$, stated in Theorem III below, are also of independent interest and have various applications. 
Main Theorem III (Moment Estimates Uniform in Volume). Let us fix any boundary condition $\xi \in \Omega$. Then for all $\delta>0, \alpha \in\left[0, \frac{1}{2}\right)$ and $Q \geq 1$,

$$
\begin{gathered}
\sup _{k \in \mathbb{Z}^{d}}\left|\xi_{k}\right|_{C_{\beta}^{\alpha}}<\infty \Longrightarrow \sup _{\Lambda \Subset \mathbb{Z}^{d}} \sup _{k \in \mathbb{Z}^{d}} \int_{\Omega}\left|\omega_{k}\right|_{C_{\beta}^{\alpha}}^{Q} \pi_{\Lambda}(d \omega \mid \xi)=: C_{Q, \xi}<\infty, \\
\xi \in \Omega_{t} \Longrightarrow \sup _{\Lambda \Subset \mathbb{Z}^{d}} \sum_{k \in \Lambda} e^{-\delta|k|} \int_{\Omega}\left|\omega_{k}\right|_{C_{\beta}^{\alpha}}^{Q} \pi_{\Lambda}(d \omega \mid \xi)=: C_{Q, \xi}^{\prime}<\infty .
\end{gathered}
$$

It should be mentioned that all the above statements extend to quantum lattice systems with general many-particle interactions (cf. Subsects. 2.5, 2.6 and 3.1-3.3 below). The proof of Theorems I-III will be given in Subsect. 5.2. A detailed analysis of the "state of the art" of the problems dealt with in the literature and a comparison of our results with the previous ones obtained by other authors will be performed in Subsect. 2.6.

2.4. Model II: Pair interaction of superquadratic growth. Here we briefly discuss how to modify the previous setting in order to include many-particle interaction potentials of superquadratic growth. Namely, let us consider the following generalization of the QLS (2.2) described by a heuristic Hamiltonian of the form

$$
\mathbb{H}:=-\frac{1}{2 \mathfrak{m}} \sum_{k \in \mathbb{Z}^{d}} \frac{d^{2}}{d q_{k}^{2}}+\frac{a^{2}}{2} \sum_{k \in \mathbb{Z}^{d}} q_{k}^{2}+\sum_{k \in \mathbb{Z}^{d}} V\left(q_{k}\right)+\sum_{\left\langle k, k^{\prime}\right\rangle \subset \mathbb{Z}^{d}} W\left(q_{k}, q_{k^{\prime}}\right) .
$$

The one-particle potential $V \in C^{2}(\mathbb{R} \rightarrow \mathbb{R})$ satisfies the same Assumption $\left(\mathbf{V}_{\mathbf{0}}\right)$ as in Subsect. 2.1, i.e., has asymptotic behaviour at infinity as a polynomial of order $P>2$. Concerning the pair potential, we suppose that $W \in C^{2}\left(\mathbb{R}^{2} \rightarrow \mathbb{R}\right)$ has respectively at most polynomial growth of the order $R<P$ :

Assumption $\left(W_{0}\right)$. There exist constants $R \in[2, P)$ and $K_{W}, C_{W}>0$ such that for all $q, q^{\prime} \in \mathbb{R}$,

$$
\left|\partial_{q}^{(l)} W\left(q, q^{\prime}\right)\right| \leq K_{W}\left(|q|^{R-l}+\left|q^{\prime}\right|^{R-l}\right)+C_{W}, \quad l=0,1,2 .
$$

Remark 2.5. A trivial example for pair potentials which fit $\left(\mathbf{W}_{\mathbf{0}}\right)$ are the polynomials $W\left(q, q^{\prime}\right):=\sum_{l=0}^{2 r}\left(q-q^{\prime}\right)^{l}$ of even degree $2 r<P$. In other words, by our assumptions is meant the so-called lattice stabilization, when the pair interaction is dominated by the single-particle one. The case $P=R$ is also allowed, but it needs a more accurate analysis, which will be performed in Sect. 3 below (in this respect see the general Assumptions $(\mathbf{V}),(\mathbf{W})$ on the interaction there).

As compared with the initial QLS model (2.2), the only principal difference in dealing with its generalization (2.21) is that we should change accordingly the notion of temperedness. Now we define the subset of tempered configurations by

$$
\Omega_{(e) t}^{R}:=\left\{\omega \in \Omega \mid \forall \delta \in(0,1):\|\omega\|_{-\delta, R}:=\left[\sum_{k \in \mathbb{Z}^{d}} e^{-\delta|k|}\left|\omega_{k}\right|_{L_{\beta}^{R}}^{2}\right]^{\frac{1}{2}}<\infty\right\} .
$$

It makes sense to consider the largest subset of such type by taking the smallest possible value of the parameter $R \geq 2$ describing the polynomial growth of the pair potential $W\left(q, q^{\prime}\right)$ in Assumption $\left(\mathbf{W}_{\mathbf{0}}\right)$. Note that for $R=2$ we just repeat the previous definition (2.6), i.e., $\Omega_{(e) t}=\Omega_{(e) t}^{R=2}$. Then all our main statements presented in Subsect. 2.3 remain true, as soon as in their formulation we substitute $L_{\beta}^{R}$ for the single spin space $L_{\beta}^{2}$ and respectively specify the subset $\mathcal{G}_{t}:=\mathcal{G}_{(e) t}^{R}$ of tempered Gibbs measures as those supported by $\Omega_{t}:=\Omega_{(e) t}^{R}$. Let us stress that (even in the case of translation invariant interactions we now deal with) we cannot guarantee that (outside the uniqueness regime) any tempered Gibbs measure will be invariant w.r.t. lattice translations. So, the above set $\mathcal{G}_{(e) t}^{R}$ is in 
a certain sense the largest one so that for any of its points $\mu$ we are technically able to get moment estimates like (2.18) uniformly w.r.t. the lattice parameter $k \in \mathbb{Z}^{d}$.

2.5. Model III: Pair interaction of infinite range. A further generalization of the QLS models (2.2) and (2.21) concerns the case of not necessarily translation-invariant pair interaction of possibly infinite range. Namely, let us consider the model described by a heuristic Hamiltonian of the form

$$
\mathbb{H}:=-\frac{1}{2 \mathfrak{m}} \sum_{k \in \mathbb{Z}^{d}} \frac{d^{2}}{d q_{k}^{2}}+\frac{a^{2}}{2} \sum_{k \in \mathbb{Z}^{d}} q_{k}^{2}+\sum_{k \in \mathbb{Z}^{d}} V_{k}\left(q_{k}\right)+\sum_{\left\{k, k^{\prime}\right\} \subset \mathbb{Z}^{d}} W_{\left\{k, k^{\prime}\right\}}\left(q_{k}, q_{k^{\prime}}\right) .
$$

The one-particle potentials $V_{k} \in C^{2}(\mathbb{R} \rightarrow \mathbb{R})$ satisfy the same Assumption $\left(\mathbf{V}_{\mathbf{0}}\right)$ as before, but with the constants $P>2$ and $K_{V}, C_{V}>0$ which are uniform for all $k \in \mathbb{Z}^{d}$. The two-particle interactions (taken over all unordered pairs $\left\{k, k^{\prime}\right\} \subset \mathbb{Z}^{d}, k \neq k^{\prime}$ ) are given by symmetric functions $W_{\left\{k, k^{\prime}\right\}} \in C^{2}\left(\mathbb{R}^{2} \rightarrow \mathbb{R}\right)$ satisfying the following growth condition:

Assumption $\left(W_{0}^{*}\right)$. There exist some constants $2 \leq R<P$ and $J_{k, j} \geq 0$ such that for all $\{k, j\} \subset \mathbb{Z}^{d}$ and $q, q^{\prime} \in \mathbb{R}$,

$$
\left|\partial_{q}^{(l)} W_{\left\{k, k^{\prime}\right\}}\left(q, q^{\prime}\right)\right| \leq J_{k, k^{\prime}}\left(1+|q|^{R-l}+\left|q^{\prime}\right|^{R-l}\right), \quad l=0,1,2 .
$$

For the matrix $\mathbf{J}:=\left\{J_{k, k^{\prime}}\right\}$ we allow different rates of decay (for instance, polynomial or exponential) when the distance $|k-j|$ between the points of the lattice gets large:

Assumption $\left(J_{0}\right)$. (i) For all $p \geq 0$,

$$
\|\mathbf{J}\|_{p}:=\sup _{k \in \mathbb{Z}^{d}}\left\{\sum_{j \in \mathbb{Z}^{d} \backslash\{k\}} J_{k, j}(1+|k-j|)^{p}\right\}<\infty
$$

holds or, even stronger,

(ii) There exists some $\delta>0$ such that

$$
\|\mathbf{J}\|_{\delta}:=\sup _{k \in \mathbb{Z}^{d}}\left\{\sum_{j \in \mathbb{Z}^{d} \backslash\{k\}} J_{k, j} e^{\delta|k-j|}\right\}<\infty .
$$

Again, we first need to choose the proper notion of temperedness, which entirely depends on the decay rate of the pair interaction. A new issue caused by the infinite range of the interaction is that one has to check (cf. Lemma 3.7 below) that the probability kernels $\pi_{\Lambda}(d \omega \mid \xi)$ are well defined for all boundary conditions $\xi \in \Omega_{t}$. So, in view of Assumption $\left(\mathbf{J}_{\mathbf{0}}\right)(\mathbf{i})$, we define the subset $\Omega_{(s) t}^{R} \subset \Omega_{(e) t}^{R}$ of ( "slowly increasing") tempered configurations by

$$
\Omega_{(s) t}^{R}:=\left\{\omega \in \Omega \mid \exists p=p(\omega)>0:\|\omega\|_{-p, R}:=\left[\sum_{k \in \mathbb{Z}^{d}}(1+|k|)^{-2 p}\left|\omega_{k}\right|_{L_{\beta}^{R}}^{2}\right]^{\frac{1}{2}}<\infty\right\} .
$$

Respectively, we introduce the subset of tempered Gibbs measures

$$
\mathcal{G}_{(s) t}^{R}:=\left\{\mu \in \mathcal{G} \mid \exists p=p(\mu)>d:\|\omega\|_{-p, R}<\infty \forall \omega \in \Omega(\bmod \mu)\right\} .
$$

Then our main Theorems I and II concerning the existence and a priori estimates for the tempered Euclidean Gibbs measures remain true, provided in their formulation one substitutes $L_{\beta}^{R}$ for the single spin spaces $L_{\beta}^{2}$ and, respectively, $\Omega_{(s) t}^{R}$ for $\Omega$ and $\mathcal{G}_{(s) t}^{R}$ for $\mathcal{G}_{t}$. In the formulation of Theorems III describing the properties of the probability kernels $\pi_{\Lambda}(d \omega \mid \xi)$ one also needs obvious changes, which we shall discuss later in Subsect. 5.2.2. On the other hand, if we want to deal with the larger subset $\mathcal{G}_{t}^{R} \supset \mathcal{G}_{(s) t}^{R}$ and completely 
keep the previous setup of the QLS Model II, we should correspondingly impose the stronger Assumption $\left(\mathbf{J}_{\mathbf{0}}\right)$ (ii) on the decay of matrix $\mathbf{J}$.

\subsection{Comments on Main Theorems I-III.}

2.6.1. Basic problems and known results. From a general viewpoint of the theory of Markov random fields, the following are fundamental problems in the study of Euclidean Gibbs measures on loop lattices:

I. Existence problem. The initial step in any study of Gibbs measures is to check whether the set $\mathcal{G}_{t}$ is nonempty. However, as is typical for systems with noncompact (in our case, even infinite-dimensional) spin spaces, the existence of $\mu \in \mathcal{G}_{t}$ stated by Theorem I is not evident at all. An important observation in this respect is that, under natural assumptions on the interaction, any accumulation point of the family $\pi_{\Lambda}, \Lambda \Subset \mathbb{Z}^{d}$, is certainly Gibbs. Depending on the specific class of quantum lattice models one deals with, the required convergence $\pi_{\Lambda^{(N)}} \rightarrow \mu, \Lambda^{(N)} \nearrow \mathbb{Z}^{d}$, and thus the existence of $\mu \in \mathcal{G}_{t}$, can be proved by the following main methods listed below:

(i) General Dobrushin's criterion for existence of Gibbs distributions [Do70]: The validity of the sufficient conditions of the Dobrushin existence theorem for some classical unbounded spin systems (including $P(\phi)$-lattice models, cf. Remark 2.1) has been verified, e.g., in COPP78, BH-K82, Sin82 (however, under assumptions on the interaction potentials more restrictive than $\left(\mathbf{V}_{0}\right)$ and $\left.\left(\mathbf{W}_{0}\right)\right)$. Contrary to the classical case, the same problem for quantum lattice systems was not covered at all by any previous work (see the discussion in BaK91]) and will be treated in one of our subsequent papers.

(ii) Ruelle's technique of superstability estimates (cf. the original papers [Rue69, LP76] and resp. [PaY94, PaY95] for its extension to the quantum case): This technique otherwise requires that the interaction is translation invariant and (according to the so-called regularity condition) the many-particle potentials have at most quadratic growth (i.e., $\left(\mathbf{W}_{\mathbf{0}}\right)$ holds with $\left.R=2\right)$. As was shown in PaY94, for the subclass of boundary conditions $\xi \in \Omega_{(s s) t} \subset \Omega_{(s) t}$ (for instance, such that $\left.\sup _{k \in \mathbb{Z}^{d}}\left|\xi_{k}\right|_{L_{\beta}^{2}}<\infty\right)$ the family of probability kernels $\pi_{\Lambda}(d \omega \mid \xi), \Lambda \Subset$ $\mathbb{Z}^{d}, \Lambda \nearrow \mathbb{Z}^{d}$, is tight (in the sense of local weak convergence on $\Omega$ ) and has at least one accumulation point $\mu$ from the subset of superstable Gibbs measures $\mathcal{G}_{(s s) t} \subset \mathcal{G}_{(s) t}$ (for the corresponding definitions, see Remark 5.14 (ii) below). This technique also shows that any $\mu \in \mathcal{G}_{(s s) t}$ is a priori of sub-Gaussian growth.

(iii) Cluster expansions is one of the most powerful methods for the study of Gibbs fields, but it works only in a perturbative regime, i.e., when an effective parameter of the interaction is small. In particular, various versions of this technique imply both existence and also uniqueness (but in some sense weaker than DLR) of the associated infinite volume Gibbs distributions (see e.g. the early works AH-K75, GJS75, GJ81, and the recent developments in PaY95, AKMR98, FM99, MRZ00, MVZ00).

(iv) The method of correlation inequalities involves more detailed information about the structure of the interaction (for instance, whether they are ferromagnetic or convex; see Remark 2.1). Starting from a number of correlation inequalities (such as FKG, GKS, Lebowitz, Brascamp-Lieb, etc.) commonly known for classical lattice systems, by a lattice approximation technique (similar to that used in Euclidean field theory) one can extend them to the quantum case (cf. OS99, AKKR01, AKKR02). 
(v) The method of reflection positivity (as a part of (iv)) applies to translation invariant systems of type (2.23) with near-neighbours pair interactions (i.e., when $V_{k}:=V, W_{\left\{k, k^{\prime}\right\}}:=W$, and $W=0$ if $\left|k-k^{\prime}\right|>1$ ). For the general description of the method and its applications to classical lattice systems we refer the reader e.g. to [Sh86]. The proper modification of this technique for the QLS (2.23) gives the existence of so-called periodic Gibbs states (for their definition see AKKR02 as well as Remark 3.10 (iv) below). Moreover, the reflection positivity method can also be used to study phase transitions in such models with the double-well anharmonicity $V$. This has been implemented under certain conditions (e.g., in the dimension $d \geq 3$ and for large enough $\beta, \mathfrak{m} \gg 1$ ) in DLP79, PK87, BaK91, HM00.

(vi) The method of stochastic dynamics (also referred to in quantum physics as "stochastic quantization"; see e.g. [Fu91, DPZ96, AKRT01] and the related bibliography therein): In this method the Gibbs measures are directly treated as invariant (more precisely, reversible) distributions for the so-called Glauber or Langevin stochastic dynamics. However, some additional technical assumptions are required on the interaction (among them at most quadratic growth of the pair potentials $\left.W_{\left\{k, k^{\prime}\right\}}\left(q, q^{\prime}\right)\right)$ in order to ensure the solvability of the corresponding stochastic evolution equations in infinite dimensions (not to mention the extremely difficult ergodicity problem for them). This method has been first applied in AKRT01 to prove existence of Euclidean Gibbs states for the particular QLS model (2.2) (see also the discussion in Subsect. 5.3 below).

II. A priori estimates for measures in $\mathcal{G}_{t}$. In turn, Theorem II above contributes to the fundamental problem of getting uniform estimates on correlation functionals of Gibbs measures in terms of parameters of the interaction. This problem was initially posed for classical lattice systems in COPP78, BH-K82 and is closely related to the compactness of the set of tempered Gibbs states (cf. Corollary after Theorem II in Subsect. 2.3); we refer also to [AKRT00] for a detailed discussion of the classical lattice case. There are very few results in the literature pertaining to a priori integrability properties of tempered Gibbs measures on loop or path spaces (see, for instance, Iw85, Fu91, OS99] for the case of Euclidean $P(\phi)_{1}$-fields and resp. AKRT01 for the case of quantum anharmonic crystals). All of them are based on the method of stochastic dynamics already mentioned above. It is worth noting that the other methods (cf. Items I. (i) $-(\mathbf{v})$ ) also give some estimates on limit points for $\pi_{\Lambda}, \Lambda \Subset \mathbb{Z}^{d}$, but not uniformly and not for all $\mu \in \mathcal{G}_{t}$. Besides, the finiteness of the moments of the measures $\mu \in \mathcal{G}_{t}$ is also useful for the study of Gibbs measures by means of the associated Dirichlet operators $\mathbb{H}_{\mu}$ in the spaces $L^{p}(\mu), p \geq 1$ (this is known as the Holley-Stroock approach HS76, AKR97a, AKR97b]). In particular, by AKR97a, AKR97b $\mu$ is an extreme point (or pure phase) in $\mathcal{G}_{t}$ if and only if the corresponding Markov semigroup $\exp \left(-t \mathbb{H}_{\mu}\right), t \geq 0$, is ergodic in $L^{2}(\mu)$ (which extends the famous result in HS76 related to the Ising model); see also a related discussion in Subsect. 5.3 below.

In this paper we shall be mainly focused on the first two problems described above, namely existence and a priori estimates for $\mu \in \mathcal{G}_{t}$. However, to give the reader a greater insight into the subject, we also mention the following important problems.

III. Uniqueness problem. The validity of the sufficient conditions of the Dobrushin uniqueness criterion (see [Do70, Fo82]) for the QLS's (2.19) with pair interactions of at most quadratic growth was first verified in [AKRT97a, AKRT97b]. In doing so, the coefficients of Dobrushin's matrix were estimated by means of log-Sobolev inequalities proved on the single loop spaces $L_{\beta}^{2}$, and the uniqueness of $\mu \in \mathcal{G}_{t}$ was established for 
small values of the inverse temperature $\beta \in\left(0, \beta_{0}\right)$, but under conditions independent of the particle mass $\mathfrak{m}>0$. For a special class of ferromagnetic models with the polynomial self-interaction (2.4), these results were essentially improved in the recent series of papers AKKR01-AKKR03. The latter papers propose a new technique, which combines the classical ideas of [LP76, BH-K82 based on the use of FKG and GKS correlation inequalities with the spectral analysis of one-site oscillators (2.1) specific for the quantum case. The strongest result of such type obtained in AKKR03 establishes the uniqueness of $\mu \in \mathcal{G}_{t}$ in the small-mass domain $\mathfrak{m} \in\left(0, \mathfrak{m}_{0}\right)$ uniformly at all values of $\beta>0$. This provides a mathematical justification for the well-known physical phenomenon that structural phase transition for a given mass $\mathfrak{m}>0$ can be suppressed not only by thermal fluctuations (i.e., high temperatures $\beta^{-1}>\beta_{c r}^{-1}$ ), but for the light particles (with $\mathfrak{m}<\mathfrak{m}_{c r}$ ) also by the quantum fluctuations (i.e., tunneling in a double-well potential) simultaneously at all temperatures $\beta>0$.

IV. Phase transitions. There are basically two general methods for proving existence of phase transitions (i.e., nonuniqueness of $\mu \in \mathcal{G}_{t}$ ) for low temperatures $\beta^{-1}$, namely, the reflection positivity (for $d \geq 3$ ) and the energy-entropy (Peierls-type) argument (for $d \geq 2$ ). However, in practice their successful applications to quantum lattice systems have been limited so far to ferromagnetic $P(\phi)$-models (cf. [DLP79, PK87, BaK91, HM00, resp. GJS75, AKRe98). The first method (already mentioned in Item I.(v)) enables one to prove the positivity of a long-range order parameter $\lim _{|\Lambda| \rightarrow \infty} E_{\mu_{p e r, \Lambda}}\left[\sum_{k \in \Lambda} \omega_{k}(\tau)\right]^{2} /$ $|\Lambda|^{2}$ for large enough $\mathfrak{m}>\mathfrak{m}_{0}$ and $\beta>\beta_{0}\left(\mathfrak{m}_{0}\right)$ via the so-called infrared (Gaussian) bounds on two-point correlation functions $E_{\mu_{p e r, \Lambda}} \omega_{k}(\tau) \omega_{k^{\prime}}(\tau)$ w.r.t. the local Gibbs measures $\mu_{p e r, \Lambda}$ with periodic boundary conditions. The second method was originally discovered (as the so-called Peierls argument) for the Ising model and further developed to apply to rather general classical spin systems (now known as the Pirogov-Sinai contour method), cf. Sin82. Its quantum modification was first implemented in GJS75] for the study of phase transition in the $\left(\varphi^{4}\right)_{2}$-model of Euclidean field theory and then in AKRe98 for its lattice approximation (2.2). Following the ideas of that paper, one defines a "collective spin variable" $\sigma_{k}:=\operatorname{sgn} \int_{S_{\beta}} \omega_{k}(\tau) d \tau$ taking values \pm 1 and a longrange parameter as the correlation function $\left\langle\sigma_{k} \sigma_{k^{\prime}}\right\rangle:=\lim _{|\Lambda| \rightarrow \infty} E_{\mu_{\Lambda}^{p e r}} \sigma_{k} \sigma_{k^{\prime}}$. The existence of long-range behaviour, and hence phase transition, follows from the estimate $\left\langle\sigma_{k} \sigma_{k^{\prime}}\right\rangle \geq 1 / 2$ valid for large enough values of $\mathfrak{m}$ and $\beta$.

V. Euclidean ground states. Of special interest for quantum systems is the case of zero absolute temperature, i.e., $\beta=\infty$, which is technically more complicated and less studied in the literature. In particular, it involves the important problem of the operator realization of the formal Hamiltonian (2.2) in quantum mechanics (cf. AH-K75, BeK93]). The corresponding Gibbs measures $\mu \in \mathcal{G}_{g r}$ on the "path lattice" $[C(\mathbb{R})]^{\mathbb{Z}^{d}}$, so-called Euclidean ground states, also allow the DLR-description, but through a family of local specifications $\pi_{I \times \Lambda}$ indexed by "time-space" windows $I \times \Lambda$ with $I \Subset \mathbb{R}, \Lambda \Subset \mathbb{Z}^{d}$; cf. MRZ00. A principal difference with the previous case $0<\beta<\infty$ is that now there is not available any such (independent from boundary conditions $\xi$ ) reference measure, so that all $\pi_{I \times \Lambda}(d \omega \mid \xi)$ are defined as its Gibbs modifications. So far, there are very few rigorous results on Gibbs measures on the path space $[C(\mathbb{R})]^{\mathbb{Z}^{d}}$, all of which are mainly related to the existence problem. Recent progress in this direction was achieved in the series of papers [FM99, MRZ00, MVZ00, where the limit measures $\lim _{\Lambda} \nearrow_{\mathbb{Z}^{d}} \lim _{I \nearrow \mathbb{R}} \pi_{I \times \Lambda} \in \mathcal{G}_{g r}$ for the $P(\phi)$-lattice models (2.2) were constructed through cluster expansions w.r.t. the small mass parameter $\mathfrak{m} \ll 1$. At the same time, for fixed $\Lambda \Subset \mathbb{Z}^{d}$, the corresponding unique Gibbs measures $\mu_{g r, \Lambda}:=\lim _{I / \mathbb{R}} \pi_{I \times \Lambda}$ on the path space $[C(\mathbb{R})]^{\Lambda}$ are well known as the $P(\phi)_{1}$-processes and can be looked upon as a special case of Euclidean field theory 
in space-dimension zero (cf. Iw85, OS99]). Furthermore, it should be noted that the Gibbs measures on the path space $[C(\mathbb{R})]^{\mathbb{Z}^{d}}$ also appear in a natural way as weak solutions for SDE's in $\mathbb{Z}^{d}$ Deu87, MRZ00.

2.6.2. Comparison with the previous results. We emphasize that our Main Theorems I and II improve essentially all the known results on the existence and a priori estimates for tempered Euclidean Gibbs measures presented in Subsect. 2.6.1 above. As already stressed in the Introduction, in order to prove our statements formulated in Subsect. 2.3, we shall propose a new technique, which completely differs from those listed under Items I.(i) $-(\mathrm{v})$ and relies on the alternative description of $\mu \in \mathcal{G}$ via integration by parts. Then our main theorems will follow immediately from corresponding results on symmetrizing measures in the abstract framework of Sects. 6, 7. Moreover, our technique obviously extends (cf. Sect. 3) to general many-particle interactions (not necessarily translation invariant and possibly having superquadratic growth, unbounded order and infinite range), which were not covered at all by any previous work. On the other hand, our approach is conceptually more straightforward and technically easier in comparison to the stochastic dynamics method mentioned under I.(vi) above.

This alternative approach was first realized in AKRT99, AKRT00, however, in the much simpler situation of classical lattice systems such as (2.14) with finite-dimensional spins. But the concrete technique suggested in those papers does not apply to loop spaces, so that a proper (highly nontrivial) modification for the quantum case is necessary. The reason is that in the quantum case we have to do not only a "lattice analysis" (depending on the properties of the interaction potentials $V, W$ ), but also an additional "single spin space analysis" (taking into account the spectral properties of the elliptic operator $A_{\beta}$ ).

It should also be mentioned that in the recent preprint Ha01 some (deterministic) version of integration by parts for local specifications has been used to prove existence of Gibbs measures relative to Brownian motion on the path space $C\left(\mathbb{R} \rightarrow \mathbb{R}^{d}\right)$. The study of such Gibbsian (in general non-Markovian) processes was initiated in OS99. As a special case they include the so-called $P(\phi)_{1}$-processes as Gibbs distributions corresponding to a single quantum particle at zero temperature, i.e., $\beta=\infty$ (see e.g. [Iw85). Finally, let us notice that our method can also be modified to apply to the case of zero absolute temperature, i.e., $\beta=\infty$, and corresponding Euclidean ground states on the "path lattice" $[C(\mathbb{R})]^{\mathbb{Z}^{d}}$ (cf. Item $\mathbf{I V}$ in Subsect. 2.6.1). This case is under present investigation.

\section{A GENERAL MODEL OF QUANTUM ANHARMONIC CRYSTALS}

In this section we describe in detail the Euclidean Gibbsian setup for our main model (3.1), which obviously includes all the particular models introduced in Section 2 and hence will be further referred to as the general $Q L S$ model.

3.1. Assumptions on the interaction. In what follows we shall consider the system of quantum anharmonic oscillators on $\mathbb{Z}^{d}$ with general (not necessarily translationinvariant) many-particle interactions (possibly of unbounded order and infinite range), which is described by the heuristic infinite-dimensional Hamiltonian

$$
\begin{aligned}
\mathbb{H}:= & -\frac{1}{2 \mathfrak{m}} \sum_{k \in \mathbb{Z}^{d}} \frac{d^{2}}{d q_{k}^{2}}+\frac{a^{2}}{2} \sum_{k \in \mathbb{Z}^{d}} q_{k}^{2}+\sum_{k \in \mathbb{Z}^{d}} V_{k}\left(q_{k}\right) \\
& +\sum_{M=2}^{N} \sum_{\left\{k_{1}, \ldots, k_{M}\right\} \subset \mathbb{Z}^{d}} W_{\left\{k_{1}, \ldots, k_{M}\right\}}\left(q_{k_{1}}, \ldots, q_{k_{M}}\right) .
\end{aligned}
$$


Notation 3.1. Below we shall distinguish between the following notation: $\left(k_{1}, \ldots, k_{M}\right) \in$ $\left(\mathbb{Z}^{d}\right)^{M}$ will stand for ordered sets (= sequences) of length $M$, and $\left\{k_{1}, \ldots, k_{M}\right\} \subset \mathbb{Z}^{d}$ for unordered sets consisting of $M$ distinct points.

Definition 3.2. We specify Assumptions $\left(\mathbf{W}_{\mathbf{i}-\mathbf{i i i}}\right),(\mathbf{J})$ and $\left(\mathbf{V}_{\mathbf{i}-\mathbf{v}}\right)$ on the system (3.1) as follows.

(W) The $M$-particle interaction potentials (taken over all sets $\left\{k_{1}, \ldots, k_{M}\right\} \subset \mathbb{Z}^{d}$ with finite $M \in\{2, \ldots, N\}$ and possibly infinite $N \in \mathbb{N} \cup\{+\infty\}$ ) are given by twice continuously differentiable symmetric functions $W_{\left\{k_{1}, \ldots, k_{M}\right\}} \in C^{2}\left(\mathbb{R}^{M} \rightarrow \mathbb{R}\right)$ satisfying a polynomial growth condition. The latter means that there exist $R \geq 2$ and symmetric matrices

$$
\mathbf{J}_{M}=\left\{J_{k_{1}, \ldots, k_{M}}\right\}_{\left(k_{1}, \ldots, k_{M}\right) \in \mathbb{Z}^{d M}}, \quad J_{k_{1}, \ldots, k_{M}} \geq 0,
$$

such that for all $M \leq N,\left\{k_{1}, \ldots, k_{M}\right\} \subset \mathbb{Z}^{d}$ and $q_{1}, \ldots, q_{M} \in \mathbb{R}$,

(i) $\left|W_{\left\{k_{1}, \ldots, k_{M}\right\}}\left(q_{1}, \ldots, q_{M}\right)\right| \leq J_{k_{1}, \ldots, k_{M}}\left(1+\sum_{l=1}^{M}\left|q_{l}\right|\right)^{R}$,

$$
\begin{aligned}
& \text { (ii) }\left|\partial_{1} W_{\left\{k_{1}, \ldots, k_{M}\right\}}\left(q_{1}, \ldots, q_{M}\right)\right| \leq J_{k_{1}, \ldots, k_{M}}\left(1+\sum_{l=1}^{M}\left|q_{l}\right|\right)^{R-1}, \\
& \text { (iii) }\left|\partial_{1} \partial_{l} W_{\left\{k_{1}, \ldots, k_{M}\right\}}\left(q_{1}, \ldots, q_{M}\right)\right| \leq J_{k_{1}, \ldots, k_{M}}\left(1+\sum_{l=1}^{M}\left|q_{l}\right|\right)^{R-2},
\end{aligned}
$$

where $\partial_{l}$ denotes derivative w.r.t. the variable $q_{l}, 1 \leq l \leq M$. Without loss of generality, we put

$$
J_{k_{1}, \ldots, k_{M}}=0 \text { if } k_{l_{1}}=k_{l_{2}} \text { for some } 1 \leq l_{1}<l_{2} \leq M .
$$

(J) The matrices $\mathbf{J}_{M}=\left\{J_{k_{1}, \ldots, k_{M}}\right\}_{k_{1}, \ldots, k_{M} \in \mathbb{Z}^{d}}, M=2, \ldots, N$, are fast decreasing; that is, for any $p \geq 0$,

$$
\|\mathbf{J}\|_{p}:=\sum_{M=2}^{N} M^{R}\left\|\mathbf{J}_{M}\right\|_{p}<\infty,
$$

where we define the following seminorms on $\mathbb{R}^{\left(\mathbb{Z}^{d}\right)^{M}}$ :

$$
\begin{gathered}
\left\|\mathbf{J}_{M}\right\|_{p}:=\sup _{k_{1} \in \mathbb{Z}^{d}}\left\{\sum_{\left\{k_{2}, \ldots, k_{M}\right\} \subset \mathbb{Z}^{d}} J_{k_{1}, \ldots, k_{M}}\left(1+\sum_{l=1}^{M}\left|k_{1}-k_{l}\right|\right)^{p}\right\}, \\
\text { e.g., }\left\|\mathbf{J}_{M}\right\|_{0}:=\sup _{k_{1} \in \mathbb{Z}^{d}}\left\{\sum_{\left\{k_{2}, \ldots, k_{M}\right\} \subset \mathbb{Z}^{d}} J_{k_{1}, \ldots, k_{M}}\right\} .
\end{gathered}
$$

(V) The anharmonic self-interactions are given by twice continuously differentiable functions $V_{k} \in C^{2}(\mathbb{R} \rightarrow \mathbb{R})$ which satisfy the following coercivity estimates with some fixed $K_{1}, K_{2}>0$, small enough $K_{3}>0$ (cf. Lemma 3.6 and Theorem 7.6), arbitrarily small $K_{4}>0$, and corresponding $L_{1}\left(K_{1}\right), \ldots, L_{4}\left(K_{4}\right)>0$ :

$$
V_{k}^{\prime}(q) \cdot q \geq \max \left\{\begin{array}{r}
K_{1}^{-1}\left(\left|V_{k}^{\prime}(q)\right|+\left|V_{k}^{\prime \prime}(q)\right|-L_{1}\right), \\
K_{2}^{-1}\left(\left|V_{k}^{\prime \prime}(q) \cdot q\right|-L_{2}\right), \\
K_{3}^{-1}\left(|q|^{R}-L_{3}\right), \\
K_{4}^{-1}\left(q^{2}-L_{4}\right)
\end{array}\right\}
$$

and the growth condition with some fixed $K_{0}, L_{0}>0$,

$$
\left|V_{k}^{\prime \prime}(q)\right| \leq K_{0}\left(\left|V_{k}^{\prime}(q)\right|+|q|^{R-1}\right)+L_{0},
$$

uniformly for all $k \in \mathbb{Z}^{d}$ and $q \in \mathbb{R}$. 
Remark 3.3. (i) Formally, no bound on the growth at infinity of the one-particle potentials is directly imposed. But, it is easy to show that $\left(\mathbf{V}_{\mathbf{i i i}}\right)$ implies that the $V_{k}$ must grow fast enough: for any $0<\sigma<\left(K_{3} R\right)^{-1}$ there exists $C_{k}:=C_{k}(\sigma) \in \mathbb{R}$ such that

$$
V_{k}(q) \geq \sigma|q|^{R}+C_{k}, \quad \text { for all } q \in \mathbb{R} .
$$

On the other hand, Assumption $\left(\mathbf{V}_{\mathbf{v}}\right)$ guarantees (by Gronwall's inequality) that with the necessity there exists $C_{k}^{\prime} \in(0, \infty)$ such that

$$
\left|V_{k}^{\prime}(q)\right| \leq C_{k}^{\prime}\left(1+|q|^{R}\right) \exp K_{0}|q|, \quad \text { for all } q \in \mathbb{R} .
$$

Typical examples of $V_{k}$ satisfying Assumptions $\left(\mathbf{V}_{\mathbf{i}-\mathbf{v}}\right)$ are linear exponential functionals or polynomials of even degree, i.e.,

$$
e^{\lambda q}+e^{-\lambda q}, \quad b_{2 n} q^{2 n}+\cdots+b_{1} q+b_{0}, \quad q \in \mathbb{R},
$$

with $\lambda \neq 0, b_{2 n}>0$ and $2 n \geq R, n \in \mathbb{N}$, as well as their products and sums.

(ii) Coercivity assumptions such as $\left(\mathbf{V}_{\text {iii }}\right)$ on potentials are standard in mathematical physics, especially when one studies stability properties of dynamical systems (for more concrete applications to the infinite-dimensional SDE's see, e.g., [DPZ96]). If $R>2$, then Assumption $\left(\mathbf{V}_{\mathbf{i v}}\right)$ itself becomes superfluous as a trivial sequel of $\left(\mathbf{V}_{\mathbf{i i i}}\right)$. Moreover, Assumptions $\left(\mathbf{V}_{\mathbf{i}-\mathbf{v}}\right)$ hold just with arbitrarily small $K_{0}, \ldots, K_{4}>0$ for each of the particular QLS models described in Sect. 2.

(iii) We say that the interaction in (3.1) is local, if it has bounded order $M \in \mathbb{N}$ and finite range $\rho \in(0, \infty)$. This means that all $W_{\left\{k_{1}, \ldots, k_{M}\right\}}=0$ whenever $M>N$ or $\operatorname{diam}\left\{k_{1}, \ldots, k_{M}\right\}>\rho$. On the other hand, the interaction is said to be translation invariant whenever $V_{k_{0}}=V$ and $W_{\left\{k_{1}, \ldots, k_{M}\right\}}=W_{\left\{k_{1}+k_{0}, \ldots, k_{M}+k_{0}\right\}}$ for all $\left\{k_{0}, k_{1}, \ldots, k_{M}\right\} \Subset \mathbb{Z}^{d}$ with $2 \leq M \leq N$.

(iv) The simplest and exactly solvable case of the QLS (3.1) is the so-called harmonic system with $N=2$ and $W_{\left\{k, k^{\prime}\right\}}\left(q_{1}, q_{2}\right):=a_{k, k^{\prime}} q_{1} q_{2}$. Here $a_{k, k^{\prime}}$ are the elements of the "dynamical matrix" $\mathbf{D}=\left(a_{k, k^{\prime}}\right)_{k, k^{\prime} \in \mathbb{Z}^{d}}$, which is usually supposed to be symmetric, bounded and strictly positive in the Hilbert space $l^{2}\left(\mathbb{Z}^{d}\right)$. Obviously, this interaction satisfies the above Assumptions (W) and (J) as soon as $\|\mathbf{D}\|_{p}<\infty, \forall p \geq 0$.

(v) It would be worthwhile to give here a nontrivial example of potentials $W_{\left\{k_{1}, \ldots, k_{M}\right\}}$, $M \in \mathbb{N}$, satisfying Assumptions $(\mathbf{W})$ and $(\mathbf{J})$ with $N=\infty$. Let

$$
W_{\left\{k_{1}, \ldots, k_{M}\right\}}:=C_{k_{1}, \ldots, k_{M}}\left(1+\sum_{\left\{k_{l_{1}}, k_{l_{2}}\right\} \subset\left\{k_{1}, \ldots, k_{M}\right\}}\left|q_{l_{1}}-q_{l_{1}}\right|^{2}\right)^{R / 2},
$$

where

$$
\begin{aligned}
C_{k_{1}, \ldots, k_{M}} & :=C_{M}^{-1} \cdot \exp \left\{-2 \sigma \sum_{\left\{k_{l_{1}}, k_{l_{2}}\right\} \subset\left\{k_{1}, \ldots, k_{M}\right\}}\left|k_{l_{1}}-k_{l_{2}}\right|\right\}, \\
C_{M} & :=M^{R+\sigma+2} R \sum_{n \in \mathbb{N}}(2 n+1)^{d M} \exp (-\sigma n)<\infty,
\end{aligned}
$$

and $\sigma>0$ is arbitrary. Then even a very rough estimate for $J_{k_{1}, \ldots, k_{M}}:=M^{2} R C_{k_{1}, \ldots, k_{M}}$ is enough to show that

$$
\left\|\mathbf{J}_{M}\right\|_{p} \leq C_{M}^{-1} \cdot \sum_{n \in \mathbb{N}}(2 n+1)^{d M}(n+1)^{p} \exp (-2 \sigma n) \leq C_{p} M^{-(R+\delta+1)}
$$

with

$$
C_{p}:=\sum_{n \in \mathbb{N}}(n+1)^{p} \exp (-\sigma n)<\infty,
$$


and thus

$$
\|\mathbf{J}\|_{p}:=\sum_{M=2}^{N} M^{R}\left\|\mathbf{J}_{M}\right\|_{p} \leq C_{p} \sum_{M=2}^{N} M^{-(\delta+1)}<\infty, \quad \text { for all } p \geq 0 .
$$

3.2. Loop spaces and the support of Euclidean Gibbs measures. Let us fix some finite value of the inverse temperature $\beta>0$. In this subsection we define the corresponding temperature loop lattices which describe configurations of the infinite volume system with Hamiltonian (3.1). It should be noted that, contrary to the classical lattice systems (2.14), in the present situation even the single spin spaces are themselves infinite dimensional (e.g., nonreflexive and nonsmooth Banach spaces) and their topological features should be taken properly into account (cf. Subsect. 3.1). Although some notation and definitions have already been used in Sect.2, for the convenience of the reader we recall them in the context of the general model (3.1).

3.2.1. One-particle loop spaces. Let $S_{\beta}$ be a circle of length $\beta$, which will be considered as a compact Riemannian manifold with Lebesgue measure $d \tau$ as a volume element and distance

$$
\rho\left(\tau, \tau^{\prime}\right):=\min \left\{\left|\tau-\tau^{\prime}\right|, \beta-\left|\tau-\tau^{\prime}\right|\right\}, \quad \tau, \tau^{\prime} \in S_{\beta} .
$$

We define some standard spaces of functions (i.e., loops) $v: S_{\beta} \rightarrow \mathbb{R}$. Namely, let $C_{\beta}^{m+\alpha}:=C^{m+\alpha}\left(S_{\beta}\right), m \in \mathbb{N} \cup\{0\}, 0 \leq \alpha<1$, denote the Banach space of all continuous loops on $S_{\beta}$ whose $m$-th derivative is $\alpha$-Hölder continuous, and which is endowed with the finite norm

$$
|v|_{C_{\beta}^{m+\alpha}}:=\sup _{\tau \in S_{\beta}} \sum_{n=0}^{m}\left|v^{(n)}(\tau)\right|+\sup _{\tau, \tau^{\prime} \in S_{\beta}, \tau \neq \tau^{\prime}} \frac{\left|v^{(m)}(\tau)-v^{(m)}\left(\tau^{\prime}\right)\right|}{\left|\tau-\tau^{\prime}\right|^{\alpha}} .
$$

Let $L_{\beta}^{r}:=L^{R}\left(S_{\beta}\right)$, resp. $W_{\beta}^{r, q}:=W^{r, q}\left(S_{\beta}\right), r \geq 1, q \in \mathbb{R}$, be the Lebesgue, resp. Sobolev, spaces (with $L_{\beta}^{r}:=W_{\beta}^{r, q=0}$ ) relative to the measure $d \tau$. These spaces can be viewed as completions of $C_{\beta}^{\infty}$ for the norms

$$
|v|_{L_{\beta}^{r}}:=\left[\int_{S_{\beta}}|v(\tau)|^{r} d \tau\right]^{1 / r} \quad \text { resp. } \quad|v|_{W_{\beta}^{r, q}}:=\left|\left(-d^{2} / d \tau^{2}+\mathbf{1}\right)^{q / 2} v\right|_{L_{\beta}^{R}} .
$$

Below we will use the following well-known embeddings:

$$
\begin{gathered}
W_{\beta}^{2, q} \subseteq_{\text {compact }} L_{\beta}^{r}, \quad q>\frac{1}{2}-\frac{1}{r}, \\
W_{\beta}^{2,1} \subseteq_{\text {compact }} C_{\beta}^{\alpha^{\prime}} \subseteq \text { compact } C_{\beta}^{\alpha}, \quad 0 \leq \alpha<\alpha^{\prime}<\frac{1}{2} .
\end{gathered}
$$

However, the reader is warned that for all positive noninteger numbers $\alpha<\alpha^{\prime}$ the Hölder spaces $C_{\beta}^{\alpha^{\prime}}$ in (3.4) are not separable and the embeddings $C_{\beta}^{\alpha^{\prime}} \subseteq C_{\beta}^{\alpha}$ are not dense.

At every site $k \in \mathbb{Z}^{d}$ of the lattice as the single spin space, resp. tangent space, we define the Banach space $C_{\beta}:=C_{\beta}^{\alpha=0}$ of all real-valued continuous loops on $S_{\beta}$ with the sup-norm $|\cdot|_{C_{\beta}}$, resp. the Hilbert space $H:=L_{\beta}^{2}$ of all square integrable loops with the $L^{2}$-norm $|\cdot|_{H}=(\cdot, \cdot)_{H}^{1 / 2}$. For the corresponding Borel $\sigma$-algebras we have $C_{\beta} \in \mathcal{B}\left(L_{\beta}^{r}\right)$ and $\mathcal{B}\left(C_{\beta}\right)=\mathcal{B}\left(L_{\beta}^{r}\right) \cap C_{\beta}$. This follows from Kuratowski's theorem (cf. Pa67, p.21, Theorem 3.9]), which says

$$
\begin{gathered}
X_{2} \in \mathcal{B}\left(X_{1}\right) \text { and } \mathcal{B}\left(X_{2}\right)=\mathcal{B}\left(X_{1}\right) \cap X_{2} \text { for all Polish spaces } X_{1} \text { and } X_{2} \\
\text { for which there is a measurable embedding } X_{2} \subseteq X_{1} \text {. }
\end{gathered}
$$

On the other hand, $C_{\beta}^{\alpha} \in \mathcal{B}\left(C_{\beta}\right)$ for all $\alpha \geq 0$, which can easily be proved by showing the measurability of $|\cdot|_{C_{\beta}^{\alpha}}$ (cf. [RS75b, Subsect. X.11] 
3.2.2. Spaces of sequences over $\mathbb{Z}^{d}$. In order to describe the behaviour of the system (3.1) when $|k| \rightarrow \infty$, we introduce some spaces of real-valued functions (i.e., sequences) on a lattice.

As usual, $\mathbb{R}^{\mathbb{Z}^{d}}$ (resp. $\mathbb{R}_{0}^{\mathbb{Z}^{d}}$ ) stands for the set of all (resp. its subset of finite) sequences over $\mathbb{Z}^{d}$. By $l^{q}(\gamma), q \geq 1$, we denote the Banach space of all sequences which are summable w.r.t. the given weight $\gamma=\left\{\gamma_{k}\right\}_{k \in \mathbb{Z}^{d}}, \gamma_{k}>0$ :

$$
l^{q}(\gamma):=l^{q}\left(\mathbb{Z}^{d} ; \gamma\right):=\left\{x=\left.\left(x_{k}\right)_{k \in \mathbb{Z}^{d}} \in \mathbb{R}^{\mathbb{Z}^{d}}|| x\right|_{l^{q}(\gamma)}:=\left[\sum_{k \in \mathbb{Z}^{d}} \gamma_{k} x_{k}^{q}\right]^{1 / q}<\infty\right\} .
$$

We shall mainly use the following two systems of weights $\gamma_{p}=\left\{\gamma_{p, k}\right\}_{k \in \mathbb{Z}^{d}}$, resp. $\gamma_{\delta}=$ $\left\{\gamma_{\delta, k}\right\}_{k \in \mathbb{Z}^{d}}$, indexed by $p \in \mathbb{R}$, resp. $\delta \in \mathbb{R}$ :

$$
\gamma_{p, k}:=(1+|k|)^{p}, \quad \gamma_{\delta, k}:=\exp (\delta|k|), \quad k \in \mathbb{Z}^{d} .
$$

In particular, $l^{2}:=l^{2}\left(\mathbb{Z}^{d} ; \gamma \equiv 1\right)$ will be the standard space of square summable sequences over $\mathbb{Z}^{d}$ with the inner product $(\cdot, \cdot)_{l^{2}}:=(\cdot, \cdot)_{0}$ and the natural orthonormal basis $e_{k}:=\left(\delta_{k-j}\right)_{j \in \mathbb{Z}^{d}}, k \in \mathbb{Z}^{d}$. For convenience, in every Hilbert space $l^{2}(\gamma)$ we fix the orthonormal basis $e_{\gamma, k}:=\gamma_{k}^{-1 / 2} e_{k}, k \in \mathbb{Z}^{d}$, so that

$$
|x|_{l^{2}(\gamma)}^{2}=\sum_{k \in \mathbb{Z}^{d}} \gamma_{k}^{-1} x_{k}^{2}=\sum_{k \in \mathbb{Z}^{d}}\left(x, e_{\gamma, k}\right)_{0}^{2}, \quad x \in l^{2}(\gamma) .
$$

Choosing $l^{2}\left(\mathbb{Z}^{d}\right)$ as the tangent Hilbert space, we next define its rigging

$$
\mathcal{E}\left(\mathbb{Z}^{d}\right) \subset \mathcal{S}\left(\mathbb{Z}^{d}\right) \subset l^{2}\left(\mathbb{Z}^{d}\right) \subset \mathcal{S}^{\prime}\left(\mathbb{Z}^{d}\right) \subset \mathcal{E}^{\prime}\left(\mathbb{Z}^{d}\right)
$$

by the following pairs of mutually dual nuclear spaces:

$$
\begin{aligned}
& \mathcal{S}\left(\mathbb{Z}^{d}\right):=\underset{p=1,2, \ldots}{\operatorname{pr} \lim } l^{2}\left(\gamma_{p}\right), \mathcal{S}^{\prime}\left(\mathbb{Z}^{d}\right):=\operatorname{ind}_{p=1,2, \ldots} l^{2}\left(\gamma_{-p}\right), \\
& \mathcal{E}\left(\mathbb{Z}^{d}\right):=\underset{\delta>0}{\operatorname{ind} \lim } l^{2}\left(\gamma_{\delta}\right), \quad \mathcal{E}^{\prime}\left(\mathbb{Z}^{d}\right):=\underset{\delta>0}{\operatorname{pr} \lim l^{2}\left(\gamma_{-\delta}\right) .}
\end{aligned}
$$

In particular, $\mathcal{S}\left(\mathbb{Z}^{d}\right)$ and $\mathcal{S}^{\prime}\left(\mathbb{Z}^{d}\right)$ are well known as the Schwartz spaces of fast decreasing, resp. slowly increasing, sequences over $\mathbb{Z}^{d}$. Obviously, one has the Hilbert-Schmidt embeddings for $p^{\prime}>p+d$, resp. $\delta^{\prime}>\delta$ :

$$
\begin{gathered}
O_{p}^{p^{\prime}}: l^{2}\left(\gamma_{p^{\prime}}\right) \rightarrow l^{2}\left(\gamma_{p}\right), \quad\left\|O_{p}^{p^{\prime}}\right\|_{H S}=\left[\sum_{k \in \mathbb{Z}^{d}}(1+|k|)^{-\left(p^{\prime}-p\right)}\right]^{1 / 2}<\infty, \\
O_{\delta}^{\delta^{\prime}}: l^{2}\left(\gamma_{\delta^{\prime}}\right) \rightarrow l^{2}\left(\gamma_{\delta}\right), \quad\left\|O_{\delta}^{\delta^{\prime}}\right\|_{H S}=\left[\sum_{k \in \mathbb{Z}^{d}} \exp \left(-\left(\delta^{\prime}-\delta\right)|k|\right)\right]^{1 / 2}<\infty .
\end{gathered}
$$

Moreover,

$$
\mathcal{S}^{\prime}\left(\mathbb{Z}^{d}\right):=\bigcup_{p \geq 1} l^{2}\left(\gamma_{-p}\right)=\bigcup_{p \geq 1} l^{1}\left(\gamma_{-p}\right), \quad \mathcal{E}^{\prime}\left(\mathbb{Z}^{d}\right):=\bigcap_{\delta>0} l^{2}\left(\gamma_{-\delta}\right)=\bigcap_{\delta>0} l^{1}\left(\gamma_{-\delta}\right)
$$

with the equivalence of the corresponding systems of weighted $l^{2}$ and $l^{1}$-norms:

$$
|x|_{l^{2}\left(\gamma_{-2 p}\right)} \leq|x|_{l^{1}\left(\gamma_{-p}\right)} \leq\left\|O_{0}^{p}\right\|_{H S}|x|_{l^{2}\left(\gamma_{-p}\right)}, \quad|x|_{l^{2}\left(\gamma_{-2 \delta}\right)} \leq|x|_{l^{1}\left(\gamma_{-\delta}\right)} \leq\left\|O_{0}^{\delta}\right\|_{H S}|x|_{l^{2}\left(\gamma_{-\delta}\right)} .
$$

Let us note that $\Omega_{c l}:=\mathbb{R}^{\mathbb{Z}^{d}}$ is the configuration space for classical lattice systems such as $(2.14)$, whereas its subspaces $\mathcal{S}^{\prime}\left(\mathbb{Z}^{d}\right)$ and $\mathcal{E}^{\prime}\left(\mathbb{Z}^{d}\right)$ are commonly used to describe the support properties of tempered Gibbs measures $\mu \in \mathcal{G}_{c l}$ (see e.g. [AKRT00]). 
3.2.3. Loop lattices. Repeating definition (2.5) above, as the configuration space for the infinite volume system (3.1) we introduce the "temperature loop lattice"

$$
\Omega:=\left[C_{\beta}\right]^{\mathbb{Z}^{d}}=\left\{\omega=\left(\omega_{k}\right)_{k \in \mathbb{Z}^{d}} \mid \omega: S_{\beta} \rightarrow \mathbb{R}^{\mathbb{Z}^{d}}, \omega_{k} \in C_{\beta}\right\} .
$$

We consider $\Omega$ as a Polish (i.e., complete separable metrizable) space with the product topology, i.e., the weakest topology such that all finite volume projections

$$
\Omega \ni \omega \mapsto \mathbb{P}_{\Lambda} \omega:=\omega_{\Lambda}:=\left(\omega_{k}\right)_{k \in \Lambda} \in\left[C_{\beta}\right]^{\Lambda}=: \Omega_{\Lambda}, \quad \Lambda \Subset \mathbb{Z}^{d},
$$

are continuous. This topology is generated by the system of seminorms $\left|\omega_{k}\right|_{C_{\beta}}, k \in \mathbb{Z}^{d}$, or, what is the same, by any one of the (mutually equivalent) metrics

$$
\rho_{-p}\left(\omega, \omega^{\prime}\right):=\left[\sum_{k \in \mathbb{Z}^{d}}(1+|k|)^{-p}\left(\frac{\left|\omega_{k}-\omega_{k}^{\prime}\right|_{C_{\beta}}^{2}}{1+\left|\omega_{k}-\omega_{k}^{\prime}\right|_{C_{\beta}}^{2}}\right)\right]^{1 / 2}, p>d .
$$

Next, we provide $\Omega$ with the corresponding Borel $\sigma$-algebra $\mathcal{B}(\Omega)$, i.e., the smallest $\sigma$ algebra containing all open sets. It is a well-known fact for product spaces that $\mathcal{B}(\Omega)$ also coincides with the $\sigma$-algebra generated by cylinder sets

$$
\left\{\omega \in \Omega \mid \omega_{\Lambda} \in \Delta_{\Lambda}\right\}, \quad \Delta_{\Lambda} \in \mathcal{B}\left(\Omega_{\Lambda}\right), \Lambda \Subset \mathbb{Z}^{d} .
$$

By $\mathcal{M}(\Omega)$ we, as usual, shall mean the family of all probability measures on $(\Omega, \mathcal{B}(\Omega)$ ). Depending on the questions under discussion, $\mathcal{M}(\Omega)$ itself will be endowed with the topologies either of weak or of locally weak convergence, which turn it into a Polish space. It is worth recalling that a sequence $\left\{\mu_{n}\right\}_{n \in \mathbb{N}} \subset \mathcal{M}(\Omega)$ weakly (resp. locally weakly) converges to $\mu \in \mathcal{M}(\Omega)$ iff $E_{\mu_{n}} f \rightarrow E_{\mu} f$, as $n \rightarrow \infty$, for all bounded continuous (and resp. local, i.e., such that $f=f\left(\mathbb{P}_{\Lambda_{0}}\right)$ with some $\left.\Lambda_{0} \Subset \mathbb{Z}^{d}\right)$ functions $f: \Omega \rightarrow \mathbb{R}$.

Because of the possibly infinite radius of interaction and unboundedness of interaction potentials, we will also need some subspaces of tempered configurations (cf. the related discussion in Subsect. 2.6 above).

In general, if $X$ is one of the single loop spaces from Subsect. 3.2.1, then $l^{q}(\gamma ; X):=$ $l^{q}\left(\mathbb{Z}^{d} \rightarrow X ; \gamma\right)$ will stand for the corresponding space of weighted sequences over $\mathbb{Z}^{d}$ with values in $X$. In particular, for $\gamma:=\gamma_{p}$ given by (3.7), we shall use the following scales of Banach spaces:

$$
\begin{aligned}
& \mathcal{L}_{-p}^{r}:=l^{2}\left(\mathbb{Z}^{d} \rightarrow L_{\beta}^{r} ; \gamma_{-p}\right)=\left\{\left.\omega \in\left[L_{\beta}^{r}\right]^{\mathbb{Z}^{d}}\left|\|\omega\|_{-p, r}^{2}:=\sum_{k \in \mathbb{Z}^{d}}(1+|k|)^{-p}\right| \omega_{k}\right|_{L_{\beta}^{r}} ^{2}<\infty\right\}, \\
& (3.13) \mathcal{C}_{-p}^{\alpha}:=l^{2}\left(\mathbb{Z}^{d} \rightarrow C_{\beta}^{\alpha} ; \gamma_{-p}\right)=\left\{\left.\omega \in \Omega\left|\|\omega\|_{-p, \alpha}^{2}:=\sum_{k \in \mathbb{Z}^{d}}(1+|k|)^{-p}\right| \omega_{k}\right|_{C_{\beta}^{\alpha}} ^{2}<\infty\right\},
\end{aligned}
$$

indexed by $p \in \mathbb{R}, r \geq 1$ and $\alpha \in[0,1 / 2)$. As a special case of (3.12) for $H:=L_{\beta}^{2}$ and $p=0$, the Hilbert space

$$
\mathcal{H}:=l^{2}\left(\mathbb{Z}^{d} \rightarrow L_{\beta}^{2}\right)=\left\{\left.\omega \in\left(L_{\beta}^{2}\right)^{\mathbb{Z}^{d}}\left|\langle\omega, \omega\rangle_{\mathcal{H}}=\|\omega\|_{\mathcal{H}}^{2}:=\sum_{k \in \mathbb{Z}^{d}}\right| \omega_{k}\right|_{H} ^{2}<\infty\right\}
$$

will be treated as the tangent space to $\Omega$.

Next we define the support spaces of Euclidean Gibbs measures

$$
\Omega_{-p}^{r}:=\Omega \cap \mathcal{L}_{-p}^{r}, \quad p>d, r \geq 1,
$$


as locally convex Polish spaces with the topology generated by the system of seminorms $\|\omega\|_{-p, r}$ and $\left|\omega_{k}\right|_{C_{\beta}}, k \in \mathbb{Z}^{d}$, or equivalently, by the metric

$$
\rho_{-p, r}\left(\omega, \omega^{\prime}\right):=\left[\sum_{k \in \mathbb{Z}^{d}}(1+|k|)^{-p}\left(\left|\omega_{k}-\omega_{k}^{\prime}\right|_{L_{\beta}^{r}}^{2}+\frac{\left|\omega_{k}-\omega_{k}^{\prime}\right|_{C_{\beta}}^{2}}{1+\left|\omega_{k}-\omega_{k}^{\prime}\right|_{C_{\beta}}^{2}}\right)\right]^{1 / 2} .
$$

Respectively, for every $k \in \mathbb{Z}^{d}$,

$$
\Omega_{-p ; k}^{r}:=\left\{\omega \in \mathcal{L}_{-p}^{r} \mid \omega_{k} \in C_{\beta}\right\}
$$

will be a Banach space with the norm

$$
\|\omega\|_{-p, r ; k}:=\|\omega\|_{-p, r}+\left|\omega_{k}\right|_{C_{\beta}} .
$$

Again, all the above spaces are equipped with their Borel $\sigma$-algebras (coinciding, due to (3.5), with the $\sigma$-algebras induced on them by $\mathcal{B}(\Omega)$ ).

In Subsect. 3.3.3 we shall start with the most extended definition of tempered Gibbs measures $\mu \in \mathcal{G}_{(s) t}^{R}$ for the QLS (3.1) as those supported on the subset of "slowly increasing" configurations

$$
\Omega_{(s) t}^{R}:=\bigcup_{p \geq 1} \Omega_{-p}^{R}=\left\{\omega \in \Omega \mid \exists p=p(\omega)>0:\|\omega\|_{-p, R}<\infty\right\} .
$$

Here the parameter $R \geq 2$ describes a possible order of polynomial growth of the interaction potentials $W_{\left\{k_{1}, \ldots, k_{M}\right\}}$ according to Assumption (W). But actually, as will be shown in the proof of our Main Theorem II (cf. Corollary 5.6 (ii) below), the initial assumption

$$
\mu\left(\Omega_{-p_{0}}^{R}\right)=1 \text { for some } p_{0}>d
$$

implies further regularity and temperedness properties for any $\mu \in \mathcal{G}_{(s) t}^{R}$, namely, that

$$
\mu\left(\mathcal{C}_{-p}^{\alpha}\right)=1 \text { for all } p>d \text { and } 0 \leq \alpha<1 / 2 .
$$

We recall that the above definition of temperedness (3.17) has already been used in the context of the particular QLS model (2.23) with the pair interaction of infinite range (cf. Subsect. 2.5). To get a more precise setting for the models (2.2) and (2.21) possessing a finite radius of interaction (cf. Subsects. 2.2-2.4), we should modify (3.15)-(3.17) by taking instead of $\mathcal{L}_{-p}^{R}$ another weighted space

$$
\mathcal{L}_{-\delta}^{R}:=l^{2}\left(\mathbb{Z}^{d} \rightarrow L_{\beta}^{R} ; \gamma_{-\delta}\right)=\left\{\left.\omega \in\left[L_{\beta}^{R}\right]^{\mathbb{Z}^{d}}\left|\|\omega\|_{-\delta, R}^{2}:=\sum_{k \in \mathbb{Z}^{d}} \exp (-\delta|k|)\right| \omega_{k}\right|_{L_{\beta}^{R}} ^{2}<\infty\right\} .
$$

Then respectively

$$
\Omega_{-\delta}^{R}:=\Omega \cap \mathcal{L}_{-\delta}^{R}, \quad \delta>0, r \geq 1,
$$

and (as was already defined by (2.17) and (2.22))

$$
\Omega_{(e) t}^{R}:=\bigcap_{\delta>0} \Omega_{-\delta}^{R}=\left\{\omega \in \Omega \mid\|\omega\|_{-\delta, R}<\infty, \quad \forall \delta>0\right\} .
$$

Actually, $\Omega_{(e) t}^{R} \supset \Omega_{(s) t}^{R}$ is the largest support set for $\mu \in \mathcal{G}_{t}$ considered so far (cf. Remark 2.4.(ii)).

If there is no confusion, we shall shorten the above notation just by writing $\Omega_{t}, \mathcal{M}_{t}$ or $\mathcal{G}_{t}$. 
3.3. DLR approach to Euclidean Gibbs measures. According to AH-K75, the Euclidean measure $\mu$ corresponding to a Gibbs state $G_{\beta}$ of the quantum lattice system with Hamiltonian (3.1) has the heuristic representation

$$
d \mu(\omega):=\frac{1}{Z} \exp \{-\mathcal{I}(\omega)\} \prod_{k \in \mathbb{Z}^{d}} d \gamma_{\beta}\left(\omega_{k}\right)
$$

Here $Z$ is the normalization factor, $\gamma_{\beta}$ is the canonical distribution on $C_{\beta}$ of the oscillator bridge process of length $\beta$, and

$$
\mathcal{I}(\omega):=\int_{S_{\beta}}\left[\sum_{M=2}^{N} \sum_{\left\{k_{1}, \ldots, k_{M}\right\} \subset \mathbb{Z}^{d}} W_{\left\{k_{1}, \ldots, k_{M}\right\}}\left(\omega_{k_{1}}(\tau), \ldots, \omega_{k_{m}}(\tau)\right)+\sum_{k \in \mathbb{Z}^{d}} V_{k}\left(\omega_{k}(\tau)\right] d \tau\right.
$$

is the formal Euclidean action functional of the system in the infinite volume $\mathbb{Z}^{d}$. In full analogy with classical statistical mechanics, a rigorous meaning can be given to the measure $\mu$ by the $D L R$ formalism as a Gibbs distribution on the lattice $\mathbb{Z}^{d}$, but with the infinite-dimensional single spin (= loop) space $C_{\beta}$. So, in Subsects. 3.3.1-3.3.2 we shall first study the corresponding family of local specifications $\pi_{\Lambda}, \Lambda \Subset \mathbb{Z}^{d}$. Thereafter, in Subsect. 3.3.3 we define the set $\mathcal{G}$ of all Gibbs measures $\mu$ on the loop lattice $\Omega$ (as the solutions to the DLR equations $\left.\mu \pi_{\Lambda}=\mu, \forall \Lambda \Subset \mathbb{Z}^{d}\right)$ and its subset $\mathcal{G}_{t}:=\mathcal{G}_{(s) t}^{R}$ of tempered (as those with physical relevance) Gibbs measures supported by $\Omega_{t}:=\Omega_{(s) t}^{R}$.

3.3.1. One-particle Euclidean measures. Let us assume that a potential $V \in C_{b, l o c}^{1}(\mathbb{R})$ satisfies the coercivity condition $\left(\mathbf{V}_{\mathbf{i i i}}\right)$ in Definition 3.2. Our aim here is to identify the following one-particle distribution:

$$
d \sigma_{\beta}(v):=\frac{1}{Z_{k}} \exp \left\{-\int_{S_{\beta}} V(v(\tau)) d \tau\right\} d \gamma_{\beta}(v)
$$

as a probability measure on the loop space $C_{\beta}$ and to collect some its properties to be used later on.

We begin with the detailed construction of the basic Gaussian measure $\gamma_{\beta}$, which corresponds to a single harmonic oscillator of mass $\mathfrak{m}>0$ and rigidity $a>0$. So, in the Hilbert space $H:=L_{\beta}^{2}$ let us consider a positive selfadjoint operator (which describes the quantum character of the system) $A_{\beta}:=-\mathfrak{m} \Delta_{\beta}+a^{2} \mathbf{1}$ with domain $\mathcal{D}\left(A_{\beta}\right):=W_{\beta}^{2,2}$. Here $\Delta_{\beta}$ is the usual Laplace-Beltrami operator on the circle $S_{\beta}$ (= maximal extension in $H$ of the differential expression $d^{2} \varphi / d \tau^{2}, \varphi \in C_{\beta}^{\infty}$ ). Let us recall that the operator $A_{\beta}$ has discrete spectrum

$$
\lambda_{n}=\left(\frac{2 \pi}{\beta} n\right)^{2} \mathfrak{m}+a^{2}, \quad n \in \mathbb{Z},
$$

and a complete orthonormal system of trigonometric functions

$$
\varphi_{n}(\tau)=\left\{\begin{array}{cc}
\left(\frac{1}{\beta}\right)^{\frac{1}{2}}, & n=0, \\
\left(\frac{2}{\beta}\right)^{\frac{1}{2}} \cos \frac{2 \pi}{\beta} n \tau, & n=1,2, \ldots, \\
-\left(\frac{2}{\beta}\right)^{\frac{1}{2}} \sin \frac{2 \pi}{\beta} n \tau, & n=-1,-2, \ldots
\end{array}\right.
$$

Obviously,

$$
\sup _{n \in \mathbb{Z}}\left|\varphi_{n}\right|_{L_{\beta}^{r}}:=\kappa_{r}:=2^{\frac{1}{2}} \beta^{\frac{1}{r}-\frac{1}{2}}, \quad 1 \leq r \leq \infty \quad \text { (i.e., } \kappa_{\infty}:=\left(2 \beta^{-1}\right)^{\frac{1}{2}} .
$$


For any $f \in L_{\beta}^{1}$ we define its Fourier and Cesàro partial sums in the standard way (cf. [Ed82]) by

$$
\mathbb{S}_{K}(f):=\sum_{|n| \leq K}\left(f, \varphi_{n}\right)_{\beta} \varphi_{n}, \quad \mathbb{M}_{K}(f):=\frac{1}{K+1} \sum_{L=0}^{K} \mathbb{S}_{L}(f), \quad K \in \mathbb{N} \cup\{0\} .
$$

Since, by the Riesz theorem, $\left\{\varphi_{n}\right\}_{n \in \mathbb{Z}}$ is a Schauder basis in every $L_{\beta}^{r}, 1<r<\infty$, there exists a finite constant $\varsigma_{r}>0$ such that $\forall f \in L_{\beta}^{r}$,

$$
\sup _{K \in \mathbb{N}}\left|\mathbb{S}_{K}(f)\right|_{L_{\beta}^{r}} \leq \varsigma_{r}|f|_{L_{\beta}^{r}} \text { and } \lim _{K \rightarrow \infty}\left|f-\mathbb{S}_{K}(f)\right|_{L_{\beta}^{r}}=0 .
$$

On the other hand, by the Fejér theorem, $\forall f \in C_{\beta}$,

$$
\sup _{K \in \mathbb{N}}\left|\mathbb{M}_{K}(f)\right|_{C_{\beta}} \leq|f|_{C_{\beta}} \text { and } \lim _{K \rightarrow \infty}\left|f-\mathbb{M}_{K}(f)\right|_{C_{\beta}}=0 .
$$

From (3.24) it follows, in particular, that the set of all trigonometric polynomials

$$
T_{\beta}:=\operatorname{lin}\left\{\varphi_{n}\right\}_{n \in \mathbb{Z}} \subset C_{\beta}^{\infty}
$$

is a domain of essential selfadjointness for $A_{\beta}$. As is well known, the corresponding semigroup $P_{t}=\exp \left(-t A_{\beta}\right), t \geq 0$, is Markovian in all spaces $C_{\beta}, L_{\beta}^{r}, r \geq 1$, and one has $(t, \tau) \longmapsto\left(P_{t} f\right)(\tau) \in C^{\infty}\left((0, \infty) \times S_{\beta}\right)$ for any $f \in L_{\beta}^{r}$.

Furthermore, from (3.24) the resolvent $A_{\beta}^{-1}$ is of trace class in $L_{\beta}^{2}$ (moreover, $\operatorname{Tr}_{H} A_{\beta}^{\alpha-1}$ $<\infty, \forall \alpha<1 / 2)$. The corresponding Green function (i.e., integral kernel of $A_{\beta}^{-1}$ )

$$
\mathfrak{G}\left(\tau, \tau^{\prime}\right):=\mathfrak{G}_{\tau}\left(\tau^{\prime}\right):=\left(A_{\beta}^{-1} \delta_{\tau}\right)\left(\tau^{\prime}\right)
$$

is given by

$$
\begin{aligned}
& \mathfrak{G}\left(\tau, \tau^{\prime}\right)=\sum_{n \in \mathbb{Z}} \lambda_{n}^{-1} \varphi_{n}(\tau) \varphi_{n}\left(\tau^{\prime}\right)=\frac{1}{\beta a^{2}}+\frac{2}{\beta} \sum_{n \in \mathbb{N}}^{\infty} \frac{\cos 2 \pi n \beta^{-1}\left(\tau-\tau^{\prime}\right)}{\left(2 \pi n \beta^{-1}\right)^{2} \mathfrak{m}+a^{2}} \\
& =\frac{\mathfrak{g}}{2}\left(e^{-\frac{a}{\sqrt{\mathrm{m}}}\left(\beta-\left|\tau-\tau^{\prime}\right|_{\mathbb{R}}\right)}+e^{-\frac{a}{\sqrt{\mathrm{m}}}\left|\tau-\tau^{\prime}\right| \mathbb{R}}\right), \quad \tau, \tau^{\prime} \in S_{\beta} .
\end{aligned}
$$

Here, for the sake of convenience, we introduce a parameter (which will be relevant, e.g., in Subsect. 5.2.1)

$$
\mathfrak{g}:=\left[2 a \sqrt{\mathfrak{m}}\left(1-e^{-\frac{a}{\sqrt{\mathfrak{m}}} \beta}\right)\right]^{-1}
$$

Then, obviously,

$$
\operatorname{Tr}_{H} A_{\beta}^{-1}=\int_{S_{\beta}} \mathfrak{G}(\tau, \tau) d \tau=\beta \mathfrak{G}_{0}(0) \leq \frac{1}{a^{2}}+\frac{\beta^{2}}{\mathfrak{m}} .
$$

Also we note the following regularity properties of $\mathfrak{G}_{\tau} \in \mathcal{D}\left(A_{\beta}^{\frac{1}{2}}\right):=W_{\beta}^{2,1}$ resulting from (3.29):

$$
\left|\mathfrak{G}_{\tau}\right|_{C_{\beta}}=\mathfrak{G}_{0}(0) \leq \mathfrak{g}, \quad\left|\mathfrak{G}_{\tau}-\mathfrak{G}_{\tau^{\prime}}\right|_{C_{\beta}} \leq \mathfrak{g} \frac{a}{\sqrt{\mathfrak{m}}} \rho\left(\tau, \tau^{\prime}\right), \quad \forall \tau, \tau^{\prime} \in S_{\beta}
$$

Now let $\gamma_{\beta}$ be the Gaussian measure on $(H, \mathcal{B}(H))$ with zero mean value and correlation operator $A_{\beta}^{-1}$, which is given uniquely by its Fourier transform

$$
\int_{H} \exp i(\varphi, v)_{H} d \gamma_{\beta}(v)=\exp \left\{-\frac{1}{2}\left(A_{\beta}^{-1} \varphi, \varphi\right)_{H}\right\}, \quad \varphi \in H
$$

Actually, the set $C_{\beta}^{\alpha}, 0 \leq \alpha<1 / 2$, of Hölder continuous loops has full measure, i.e., $\gamma_{\beta}\left(C_{\beta}^{\alpha}\right)=1$, and the measure $\gamma_{\beta}$ on the space $\left(C_{\beta}, \mathcal{B}\left(C_{\beta}\right)\right)$ can be viewed as the canonical realization of the well-known oscillator bridge process of length $\beta$ (see, e.g., Sim79, 
p. 43]). Hence, the abstract Fernique's theorem (see, e.g. DeuS89, Theorem 1.3.24]) applied to $\gamma_{\beta}$ immediately gives us that for any $\alpha \in[0,1 / 2)$ there exists $\lambda_{0}:=\lambda_{0}(\alpha)>0$ such that

$$
\int_{C_{\beta}} \exp \left\{\lambda|v|_{C_{\beta}^{\alpha}}^{2}\right\} d \gamma_{\beta}(v)<\infty, \quad \forall \lambda \in\left[0, \lambda_{0}\right]
$$

Now, we can define by the Feynman-Kac formula (3.22) the probability measure $\sigma_{\beta}$ on $\left(C_{\beta}, \mathcal{B}\left(C_{\beta}\right)\right)$. By Remark 3.3.(i), the coercivity Assumption ( $\left.\mathbf{V}_{\mathbf{i i i}}\right)$ implies the polynomial growth of $V$ at infinity and, hence, its semiboundedness from below, i.e., $\inf _{\mathbb{R}} V \geq C>-\infty$. Therefore

$$
0<Z:=\int_{C_{\beta}} \exp \left\{-\int_{S_{\beta}} V(v(\tau)) d \tau\right\} d \gamma_{\beta}(v)<\exp (-\beta C)<\infty,
$$

and the definition (3.22) makes sense. Moreover, the measure $\sigma_{\beta}$ has all moments of the form

$$
\int_{C_{\beta}} \exp \left\{\lambda|v|_{L_{\beta}^{R}}^{R}\right\}\left(1+|v|_{C_{\beta}^{\alpha}}^{Q}\right) d \sigma_{\beta}(v)<\infty, 0 \leq \lambda<\left(K_{3} R\right)^{-1}, 0 \leq \alpha<\frac{1}{2}, Q \geq 1,
$$

due to (3.2) and the corresponding property (3.32) of the Gaussian measure $\gamma_{\beta}$.

An important fact is the subsequent observation that $\sigma_{\beta}$ is quasi-invariant w.r.t. shifts

$$
v \rightarrow v+\theta \varphi_{n}, \quad \theta \in \mathbb{R}, n \in \mathbb{Z}
$$

with the Radon-Nikodým derivatives

$$
\frac{d \sigma_{\beta}\left(v+\theta \varphi_{n}\right)}{d \sigma_{\beta}(v)}=: a_{\theta \varphi_{n}}(v)=a_{\theta \varphi_{n}}^{A_{\beta}}(v) a_{\theta \varphi_{n}}^{V}(v),
$$

where

$$
\begin{gathered}
a_{\theta \varphi_{n}}^{A_{\beta}}(v):=\exp \left\{-\theta\left(A_{\beta} \varphi_{n}, v\right)_{H}-\frac{\theta^{2}}{2}\left(A_{\beta} \varphi_{n}, \varphi_{n}\right)_{H}\right\}, \\
a_{\theta \varphi_{n}}^{V}(v):=\exp \left\{-\int_{S_{\beta}}\left[V\left(v(\tau)+\theta \varphi_{n}(\tau)\right)-V(v(\tau)] d \tau\right\} .\right.
\end{gathered}
$$

If, moreover, $V \in C_{b, l o c}^{2}(\mathbb{R})$, then for all $\theta \in \mathbb{R}$,

$$
\frac{d}{d \theta} a_{\theta \varphi_{n}}(v)=a_{\theta \varphi_{n}}(v)\left[\left(A_{\beta} \varphi_{n}, v+\theta \varphi_{n}\right)_{H}+\left(V^{\prime}\left(v+\theta \varphi_{n}\right), \varphi_{n}\right)_{H}\right]
$$

exists and the functions

$$
(v, \theta) \longmapsto a_{\theta \varphi_{n}}(v), \frac{d}{d \theta} a_{\theta \varphi_{n}}(v)
$$

are uniformly Lipschitz continuous on balls in $C_{\beta} \times \mathbb{R}$. As a special case of (3.36) when $\theta=0$, we define the so-called partial logarithmic derivatives $b_{\varphi_{n}}: C_{\beta} \rightarrow \mathbb{R}$ of the measure $\sigma_{\beta}$ along the basis directions $\varphi_{n}$, i.e.,

$$
b_{\varphi_{n}}(v):=\left(a_{\theta \varphi_{n}}(v)\right)_{\theta=0}^{\prime}=\left(A_{\beta} \varphi_{n}, v\right)_{H}+\left(V^{\prime}(v), \varphi_{n}\right)_{H} .
$$

Note that by construction $a_{\theta \varphi_{n}} \in L^{1}\left(\sigma_{\beta}\right)$ always. In contrast, the global integrability properties of $b_{\varphi_{n}}$ are not known in advance. In order to ensure them, we need additional assumptions on the asymptotic behaviour of the potential, for instance, that $V^{\prime}$ satisfies the polynomial growth condition

$$
\left|V^{\prime}(q)\right| \leq C^{\prime}\left(1+|q|^{R}\right), \quad q \in \mathbb{R} .
$$


Then from the moment estimate (3.33) one can easily conclude that for small enough $\theta_{0} \in(0, \infty)$

$$
\sup _{0 \leq \theta \leq \theta_{0}}\left|(d / d \theta) a_{\theta \varphi_{n}}\right| \in L^{Q}\left(\sigma_{\beta}\right) \text {, and hence also } b_{\varphi_{n}} \in L^{Q}\left(\sigma_{\beta}\right) .
$$

If (3.39) holds at least for $Q=1$, then (3.36) in turn implies (by Lebesgue's theorem applied to (4.23) below) the following integration by parts formula

$$
\int_{C_{\beta}}(d / d \theta) f\left(v+\theta \varphi_{n}\right) d \sigma_{\beta}(v)=-\int_{C_{\beta}} f(v) b_{\varphi_{n}}(v) d \sigma_{\beta}(v)
$$

valid on all smooth cylinder functions $f(v):=f_{L}\left(\left(v, \varphi_{1}\right)_{H}, \ldots,\left(v, \varphi_{L}\right)_{H}\right)$ with $f_{L} \in$ $C_{b}^{1}\left(\mathbb{R}^{L}\right)$ and $L \in \mathbb{N}$. In this case one says that the measure $\sigma_{\beta}$ is differentiable (e.g. in the well-known sense of Fomin and of Skorohod, cf. [Bo97, Sk84]) along vectors $\varphi_{n}$ with logarithmic derivatives $b_{\varphi_{n}} \in L^{1}\left(\sigma_{\beta}\right)$.

3.3.2. Local specification. The specification $\pi=\left\{\pi_{\Lambda}\right\}_{\Lambda \Subset \mathbb{Z}^{d}}$ (corresponding to the Hamiltonian (3.1)) is defined as a family of measure kernels

$$
\mathcal{B}(\Omega) \times \Omega \ni(\Delta, \xi) \rightarrow \pi_{\Lambda}(\Delta \mid \xi) \in[0,1]
$$

in the following way:

$$
\pi_{\Lambda}(\Delta \mid \xi):= \begin{cases}Z_{\Lambda}^{-1}(\xi) \int_{\Omega_{\Lambda}} \exp \left\{-\mathcal{I}_{\Lambda}^{W}(\omega \mid \xi)\right\} \mathbf{1}_{\Delta}\left(\omega_{\Lambda}, \xi_{\Lambda^{c}}\right) \prod_{k \in \Lambda} d \sigma_{\beta, k}\left(\omega_{k}\right), & \xi \in \Omega_{t}, \\ 0, & \xi \in \Omega \backslash \Omega_{t}\end{cases}
$$

(where $\mathbf{1}_{\Delta}$ denotes the indicator on $\Delta$ ). Here $\sigma_{\beta, k}$ are the one-particle Euclidean measures on $C_{\beta}$ given by (3.22) with $V:=V_{k}$,

$$
Z_{\Lambda}(\xi):=\int_{\Omega_{\Lambda}} \exp \left\{-\mathcal{I}_{\Lambda}^{W}(\omega \mid \xi)\right\} \prod_{k \in \Lambda} d \sigma_{\beta}\left(\omega_{k}\right)
$$

is the normalization factor (the so-called partition function), and

$$
\mathcal{I}_{\Lambda}^{W}(\omega \mid \xi):=\sum_{M=2}^{N} \sum_{L=1}^{M} \sum_{\substack{\left\{k_{1}, \ldots, k_{L}\right\} \subset \Lambda \\\left\{k_{L+1}, \ldots, k_{M}\right\} \subset \Lambda^{c}}} \int_{S_{\beta}} W_{\left\{k_{1}, \ldots, k_{M}\right\}}\left(\omega_{k_{1}}, \ldots, \omega_{k_{L}}, \xi_{k_{L+1}}, \ldots, \xi_{k_{M}}\right) d \tau
$$

is the many-particle interaction in the volume $\Lambda$ under the boundary condition $\xi \in \Omega_{t}:=$ $\Omega_{(s) t}^{R}$ (by convention $\left\{k_{L+1}, \ldots, k_{M}\right\}=\varnothing$ if $L \geq M$ ).

More precisely, $\pi$ is a $\left\{\mathcal{B}\left(\Omega_{\Lambda^{c}}\right)\right\}_{\Lambda \Subset \mathbb{Z}^{d}}$-specification in the sense of $\operatorname{Pr} 76$, Ge88, which means that the following properties hold for all $\Lambda \subset \Lambda^{\prime} \Subset \mathbb{Z}^{d}$ :

$\left(\mathbf{S}_{\mathbf{i}}\right) \pi_{\Lambda}(\Omega \mid \xi)$ is either 0 or 1 for all $\xi \in \Omega$;

$\left(\mathbf{S}_{\mathbf{i i}}\right) \pi_{\Lambda}(\Delta \mid \cdot)$ is $\mathcal{B}\left(\Omega_{\Lambda^{c}}\right)$-measurable for all $\Delta \in \mathcal{B}(\Omega)$;

$\left(\mathbf{S}_{\mathbf{i i i}}\right) \int_{\Omega} f(\omega) g(\omega) \pi_{\Lambda}(d \omega \mid \xi)=f(\xi) \int_{\Omega} g(\omega) \pi_{\Lambda}(d \omega \mid \xi)$ for all bounded and $\mathcal{B}\left(\Omega_{\Lambda^{c}}\right)$ (resp. $\mathcal{B}(\Omega)$ )-measurable functions $f$ (resp. $g$ ) on $\Omega$;

$\left(\mathbf{S}_{\mathrm{iv}}\right)$ Consistency: $\pi_{\Lambda^{\prime}}=\pi_{\Lambda^{\prime}} \pi_{\Lambda}$, where the kernel $\pi_{\Lambda^{\prime}} \pi_{\Lambda}$ is defined by

$$
\left(\pi_{\Lambda^{\prime}} \pi_{\Lambda}\right)(\Delta \mid \xi):=\int_{\Omega} \pi_{\Lambda^{\prime}}(d \omega \mid \xi) \pi_{\Lambda}(\Delta \mid \omega), \quad \forall \xi \in \Omega, \forall \Delta \in \mathcal{B}(\Omega) .
$$

Actually, by (3.41) every $\pi_{\Lambda}(d \omega \mid \xi)$ is concentrated on configurations of the form $\omega=$ $\left(\omega_{\Lambda}, \xi_{\Lambda^{c}}\right) \in \Omega_{t}$ whenever $\xi \in \Omega_{t}$. For each $\Lambda \Subset \mathbb{Z}^{d}$ and $\xi \in \Omega_{t}$, it is also reasonable to 
consider the so-called Gibbs distribution in the volume $\Lambda$ with the boundary condition $\xi_{\Lambda^{c}}$ as the probability measure

$$
\mu_{\Lambda}\left(d \omega_{\Lambda} \mid \xi_{\Lambda^{c}}\right):=Z_{\Lambda}^{-1}(\xi) \exp \left\{-\mathcal{I}_{\Lambda}^{W}(\omega \mid \xi)\right\} \prod_{k \in \Lambda} d \sigma_{\beta, k}\left(\omega_{k}\right)
$$

on the loop space $\Omega_{\Lambda}=\left[C_{\beta}\right]^{\Lambda}$. Equivalently, $\mu_{\Lambda}\left(d \omega_{\Lambda} \mid \xi_{\Lambda^{c}}\right)$ is the projection of $\pi_{\Lambda}(d \omega \mid \xi)$ onto $\Omega_{\Lambda}$, i.e., $\mu_{\Lambda}\left(\cdot \mid \xi_{\Lambda^{c}}\right)=\pi_{\Lambda}(\cdot \mid \xi) \circ \mathbb{P}_{\Lambda}^{-1}$.

Because of the possibly infinite range of interaction, some verification (see Lemmas 3.4 and 3.6 below) is needed of whether definitions (3.41) and (3.44) make sense, i.e., axioms $\left(\mathbf{S}_{\mathbf{i}-\mathbf{i i i}}\right)$ hold. The validity of the consistency axiom $\left(\mathbf{S}_{\mathbf{i v}}\right)$ for our model follows then from the additive structure of the functional $\mathcal{I}_{\Lambda}^{W}$ (cf. [Ge88]).

Before proceeding further, we collect here a few technical estimates on the matrix $\mathbf{J}$ to be repeatedly used below:

Lemma 3.4. (i) Let us define a symmetric matrix $\tilde{\mathbf{J}}=\left\{\tilde{J}_{k, j}\right\}_{k, j \in \mathbb{Z}^{d}}$ with the nonnegative entries

$$
\left.\tilde{J}_{k, j}:=\sum_{M=2}^{N} M^{R} \sum_{\substack{\left\{k_{1}, \ldots, k_{M}\right\} \subset \mathbb{Z}^{d} \\ k_{1}:=k, k_{2}:=j}} J_{k_{1}, \ldots, k_{M}} \quad \text { (i.e., } \tilde{J}_{k, j}:=0 \text { for } k=j\right) .
$$

Then $\forall p \geq 0$,

$$
\|\tilde{\mathbf{J}}\|_{p}:=\sup _{k \in \mathbb{Z}^{d}}\left\{\sum_{j \in \mathbb{Z}^{d}} \tilde{J}_{k, j}(1+|k-j|)^{p}\right\} \leq \sum_{M=2}^{N} M^{R}\left\|\mathbf{J}_{M}\right\|_{p}=\|\mathbf{J}\|_{p}<\infty .
$$

(ii) Let us introduce one more system of seminorms on $\mathbb{R}^{\left(\mathbb{Z}^{d}\right)^{M}}$ by

$$
\left\|\mathbf{J}_{M} \mid\right\|_{p}:=\sup _{k_{1} \in \mathbb{Z}^{d}}\left\{\sum_{\left\{k_{2}, \ldots, k_{M}\right\} \subset \mathbb{Z}^{d}} J_{k_{1}, \ldots, k_{M}} \sum_{l=1}^{M}\left(1+\left|k_{1}-k_{l}\right|\right)^{p}\right\}, \quad p \geq 0 .
$$

Then $\left\|\mathbf{J}_{M}\right\|_{p}$ and $\left\|\mathbf{J}_{M} \mid\right\|_{p}$ are equivalent, i.e.,

$$
\left\|\mathbf{J}_{M}\right\|_{0}=M\left\|\mathbf{J}_{M}\right\|_{0} \quad \text { and } \quad \frac{1}{M}\left\|\mid \mathbf{J}_{M}\right\|_{p} \leq\left\|\mathbf{J}_{M}\right\|_{p} \leq M^{p-1}\left\|\mathbf{J}_{M}\right\|_{p}, \quad p \geq 1 .
$$

(iii) Given any $\Lambda \Subset \mathbb{Z}^{d}$, let us define seminorms

$$
\left\|\mathbf{J}_{M} \mid\right\|_{\Lambda, p}:=\sum_{L=1}^{M} \sum_{\substack{\left\{k_{1}, \ldots, k_{L}\right\} \subset \Lambda \\\left\{k_{L+1}, \ldots, k_{M}\right\} \subset \Lambda^{c}}} J_{k_{1}, \ldots, k_{M}} \sum_{l=1}^{M}\left(1+\left|k_{l}\right|\right)^{p}, \quad p \geq 0 .
$$

Then

$$
\left\|\mathbf{J}_{M}\right\|\left\|_{\Lambda, p} \leq C(M, \Lambda, p) \cdot\right\| \mathbf{J} \|_{p} \text { with } C(M, \Lambda, p):=M|\Lambda| \cdot \sup _{k \in \Lambda}\left\{(1+|k|)^{p}\right\} .
$$

Proof. (i) (3.46) follows by a direct calculation from assumption (J).

(ii) (3.48) is readily apparent from (3.47) and the inequality

$$
\frac{1}{M} \sum_{l=1}^{M}\left|q_{l}\right|^{p} \leq\left(\sum_{l=1}^{M}\left|q_{l}\right|\right)^{p} \leq M^{p-1} \sum_{l=1}^{M}\left|q_{l}\right|^{p},
$$

which is valid for any $p \geq 1$ and $q_{1}, \ldots, q_{M} \in \mathbb{R}$. 
(iii) (3.50) follows from $(\mathbf{J})$ and $(3.48)$ by the estimate

$$
\begin{aligned}
\left\|\mathbf{J}_{M} \mid\right\|_{\Lambda, p} & \leq|\Lambda| \sup _{k_{1} \in \Lambda}\left\{\left(1+\left|k_{1}\right|\right)^{p}\right\} \sup _{k_{1} \in \Lambda}\left\{\sum_{\left\{k_{2}, \ldots, k_{M}\right\} \subset \mathbb{Z}^{d}} J_{k_{1}, \ldots, k_{M}} \sum_{l=1}^{M}\left(1+\left|k_{1}-k_{l}\right|\right)^{p}\right\} \\
& \leq|\Lambda| \sup _{k \in \Lambda}\left\{( 1 + | k | ) ^ { p } \left|\left\|\mathbf{J}_{M} \mid\right\|_{p} .\right.\right.
\end{aligned}
$$

Lemma 3.5. Along with $\left(\mathbf{W}_{\boldsymbol{i}}\right)$ and $(\mathbf{J})$, also let Assumption $\left(\mathbf{V}_{i i i}\right)$ hold with some fixed, but small enough $0<K_{3}<K_{3}^{(0)}$ (e.g., satisfying (3.55) below). Then

$$
0<Z_{\Lambda}(\xi)<\infty \quad \text { for all } \xi \in \Omega_{t} \text { and } \Lambda \Subset \mathbb{Z}^{d}
$$

i.e., the right-hand sides in (3.39) and (3.42) make sense.

Proof. Let $\xi \in \Omega_{-p}^{R}$ for some $p>d$. Due to $\left(\mathbf{W}_{\mathbf{i}}\right),(\mathbf{J})$ and (3.49)-(3.51) we have that

$$
\begin{aligned}
& \sum_{M=2}^{N} \sum_{L=1}^{M} \sum_{\substack{\left\{k_{1}, \ldots, k_{L}\right\} \subset \Lambda \\
\left\{k_{L+1}, \ldots, k_{M}\right\} \subset \Lambda^{c}}} \int_{S_{\beta}}\left|W_{\left\{k_{1}, \ldots, k_{M}\right\}}\left(\omega_{k_{1}}, \ldots, \omega_{k_{M}}\right)\right| d \tau \\
& \leq \sum_{M=2}^{N}(M+1)^{R-1} \sum_{L=1}^{M} \sum_{\substack{\left\{k_{1}, \ldots, k_{L}\right\} \subset \Lambda \\
\left\{k_{L+1}, \ldots, k_{M}\right\} \subset \Lambda^{c}}} J_{k_{1}, \ldots, k_{M}}\left(\beta+\sum_{l=1}^{M}\left|\omega_{k_{l}}\right|_{L_{\beta}^{R}}^{R}\right) \\
& \leq\left[\sum_{M=2}^{N}(M+1)^{R}\left\|\mathbf{J}_{M}\right\|_{p R}\right]|\Lambda| \cdot \sup _{k \in \Lambda}\left\{(1+|k|)^{p R}\right\}\left(\beta+\|\omega\|_{-p, R}^{R}\right) \\
& \leq 2^{R}\|\mathbf{J}\|_{p R}|\Lambda| \cdot \sup _{k \in \Lambda}\left\{(1+|k|)^{p R}\right\}\left(\beta+\|\omega\|_{-p, R}^{R}\right) .
\end{aligned}
$$

Thus, returning to the notation $(3.39)-(3.41)$,

$$
\begin{aligned}
\left|\mathcal{I}_{\beta, \Lambda}^{W}(\omega \mid \xi)\right| \leq & \sum_{M=2}^{N}(M+1)^{R-1}\left\|\mathbf{J}_{M}\right\|_{0} \sum_{k \in \Lambda}\left|\omega_{k}\right|_{L_{\beta}^{R}}^{R} \\
& +2^{R}\|\mathbf{J}\|_{p R}|\Lambda| \cdot \sup _{k \in \Lambda}\left\{(1+|k|)^{p R}\right\}\left(\beta+\left\|\xi_{\Lambda^{c}}\right\|_{-p, R}^{R}\right) .
\end{aligned}
$$

Combining (3.54) and (3.33), we can get even more than stated in Lemma 3.5. Namely, if

$$
0<K_{3}<K_{3}^{(0)}:=\left(2^{R-2} R \cdot\|\mathbf{J}\|_{0}\right)^{-1},
$$

then the local partition function $Z_{\Lambda}(\xi)$ is uniformly positive and bounded on all finite radius balls

$$
B_{-p}^{R}(\rho):=\left\{\xi \in \mathcal{L}_{-p}^{R} \mid\|\xi\|_{-p, R} \leq \rho\right\}, \quad 0<\rho<\infty,
$$

i.e., it follows that

$$
0<\inf _{\xi \in B_{-p}^{R}(\rho)} Z_{\Lambda}(\xi) \leq \sup _{\xi \in B_{-p}^{R}(\rho)} Z_{\Lambda}(\xi)<\infty
$$

Lemma 3.6. Under the assumptions of Lemma 3.5, the specification $\pi=\left\{\pi_{\Lambda}\right\}_{\Lambda \Subset \mathbb{Z}^{d}}$ possesses the following regularity properties: for any $\Lambda \Subset \mathbb{Z}^{d}, p>d$,

$$
(\omega, \xi) \mapsto \mathcal{I}_{\Lambda}(\omega \mid \xi) \text { is uniformly Lipschitz continuous on balls in } \mathcal{L}_{-p}^{R} \times \mathcal{L}_{-p}^{R}
$$


and hence for any bounded measurable $f: \Omega \rightarrow \mathbb{R}$,

$$
\xi \mapsto\left(\pi_{\Lambda} f\right)(\xi):=\int_{\Omega} f(\omega) \pi_{\Lambda}(d \omega \mid \xi) \text { is uniformly Lipschitz continuous on balls in } \mathcal{L}_{-p}^{R} \text {. }
$$

Proof. (3.57) is a consequence of the estimate

$$
\begin{aligned}
& \sum_{M=2}^{N} \sum_{L=1}^{M} \sum_{\substack{\left\{k_{1}, \ldots, k_{L}\right\} \subset \Lambda \\
\left\{k_{L+1}, \ldots, k_{M}\right\} \subset \Lambda^{c}}} \int_{S_{\beta}}\left|W_{\left\{k_{1}, \ldots, k_{M}\right\}}\left(\omega_{k_{1}}, \ldots, \omega_{k_{M}}\right)-W_{\left\{k_{1}, \ldots, k_{M}\right\}}\left(\omega_{k_{1}}^{\prime}, \ldots, \omega_{k_{M}}^{\prime}\right)\right| d \tau \\
\leq & \sum_{M=2}^{N} \sum_{L=1}^{M} \sum_{\substack{\left\{k_{1}, \ldots, k_{L}\right\} \subset \Lambda \\
\left\{k_{L+1}, \ldots, k_{M}\right\} \subset \Lambda^{c}}} J_{k_{1}, \ldots, k_{M}} \int_{S_{\beta}} \sum_{l=1}^{M}\left|\omega_{k_{l}}-\omega_{k_{l}}^{\prime}\right|\left(1+\sum_{l=1}^{M}\left|\omega_{k_{l}}\right|+\sum_{l=1}^{M}\left|\omega_{k_{l}}^{\prime}\right|\right)^{R-1} d \tau \\
& \leq 3^{R}\|\mathbf{J}\|_{p R}^{R}|\Lambda| \cdot \sup _{k \in \Lambda}\left\{(1+|k|)^{p R}\right\}\left\|\omega-\omega^{\prime}\right\|_{-p, R}\left(\beta^{\left(1-R^{-1}\right)}+\|\omega\|_{-p, R}^{R-1}+\left\|\omega^{\prime}\right\|_{-p, R}^{R-1}\right),
\end{aligned}
$$

which one can easily get from $\left(\mathbf{W}_{\mathbf{i i}}\right),(\mathbf{J})$, Hölder's inequality and claim (iii) of Lemma 3.4. Thereafter, (3.58) immediately follows from (3.42), (3.56) and (3.57).

Remark 3.7. In fact, the proof of Lemma 3.6 shows that, at least for small enough $\lambda, K_{3}>0$ such that

$$
K_{3}<\left[R\left(\lambda+2^{R-2}\|\mathbf{J}\|_{0}\right)\right]^{-1}=: K_{3}^{(\lambda)}<K_{3}^{(0)},
$$

the measures $\pi_{\Lambda}(d \omega \mid \xi)$ certainly have all moments of the form

$$
\int_{\Omega} \exp \left\{\lambda\left|\omega_{k}\right|_{L_{\beta}^{R}}^{R}\right\}\left(1+\left|\omega_{k}\right|_{C_{\beta}^{\alpha}}^{Q}\right) d \pi_{\Lambda}(d \omega \mid \xi)<\infty, \quad 0 \leq \alpha<\frac{1}{2}, Q \geq 1 .
$$

So, (3.60) holds for all $\lambda>0$ for the particular QLS models described in Sect. 2 (cf. Remark 3.3 (ii)).

3.3.3. Definition of tempered Euclidean Gibbs measures. In this subsection assume that $\left(\mathbf{W}_{\mathbf{i}}\right),(\mathbf{J})$ and $\left(\mathbf{V}_{\mathbf{i i i}}\right)$ in Definition 2.2 hold.

Definition 3.8. A probability measure $\mu$ on $(\Omega, \mathcal{B}(\Omega))$ is called a Euclidean Gibbs state for the specification $\pi=\left\{\pi_{\Lambda}\right\}_{\Lambda \Subset \mathbb{Z}^{d}}$ (corresponding to the lattice system (3.1) at inverse temperature $\beta>0$ ) if it satisfies the DLR equilibrium equations:

$$
\mu \pi_{\Lambda}=\mu, \forall \Lambda \Subset \mathbb{Z}^{d},
$$

where the measures $\mu \pi_{\Lambda}$ are defined by

$$
\mu \pi_{\Lambda}(\Delta):=\int_{\Omega} \mu(d \omega) \pi_{\Lambda}(\Delta \mid \omega), \quad \forall \Delta \in \mathcal{B}(\Omega) .
$$

In other words, $\mu$ is a measure on $(\Omega, \mathcal{B}(\Omega))$ such that its regular conditional distributions given $\omega_{k}, k \in \Lambda^{c}$, coincide $\mu$-a.e. with the prescribed local specifications $\pi_{\Lambda}$ for all $\Lambda \Subset \mathbb{Z}^{d}$. Let $\mathcal{G}$ denote the set of all Gibbs measures for the system (3.1) at a fixed inverse temperature $\beta>0$. Applying (3.62) to $B:=\left(\Omega_{t}\right)^{c}$, it follows from (3.41) that $\mu\left(\Omega_{t}\right)=1$, i.e., any $\mu \in \mathcal{G}$ is supported by $\Omega_{t}:=\Omega_{(s) t}^{R}:=\bigcup_{p>0} \Omega_{-p}^{R}$. For technical reasons (which become more clear after the proof of our main Hypotheses $(\mathbf{H})$ in Sect.7), we shall mainly restrict our consideration to tempered Gibbs measures $\mu \in \mathcal{G}_{t}:=\mathcal{G}_{(s) t}^{R}$ supported on the entire spaces $\Omega_{-p}^{R}, p>d$. 
Definition 3.9. A probability measure $\mu$ on $(\Omega, \mathcal{B}(\Omega)$ ) is called (exponentially) tempered, i.e., $\mu \in \mathcal{M}_{(e) t}^{R}$, if

$$
\forall \delta>0: \quad \mu\left(\Omega_{-\delta}^{R}\right)=1,
$$

and, moreover, (slowly) tempered, i.e., $\mu \in \mathcal{M}_{(s) t}^{R} \subseteq \mathcal{M}_{(e) t}^{R}$, if

$$
\exists p=p(\mu)>d: \quad \mu\left(\Omega_{-p}^{R}\right)=1 .
$$

Respectively we define the subsets $\mathcal{G}_{(s) t}^{R} \subseteq \mathcal{G}_{(e) t}^{R}$ of tempered Gibbs measures by

$$
\mathcal{G}_{(e) t}^{R}:=\mathcal{G} \cap \mathcal{M}_{(e) t}^{R}, \quad \mathcal{G}_{(s) t}^{R}:=\mathcal{G} \cap \mathcal{M}_{(s) t}^{R} .
$$

Remark 3.10. (i) In fact (cf., e.g., Ge88, Theorem 1.33]), under the conditions imposed, any tempered $\mu \in \mathcal{G}$ is completely determined just by the family of its one-site conditional distributions $\pi_{\Lambda_{k}}$ with $\Lambda_{k}:=\{k\}, k \in \mathbb{Z}^{d}$. More precisely, a probability measure $\mu$ on $\left(\Omega_{(s) t}^{R}, \mathcal{B}\left(\Omega_{(s) t}^{R}\right)\right)$ is Gibbs for $\pi=\left\{\pi_{\Lambda}\right\}_{\Lambda \Subset \mathbb{Z}^{d}}$ iff $\mu \pi_{\Lambda_{k}}=\mu$ for all $k \in \mathbb{Z}^{d}$.

(ii) Under our assumptions every cluster point $\mu_{*}$ of the family $\left\{\pi_{\Lambda}(d \omega \mid \xi)\right\}_{\Lambda \Subset \mathbb{Z}^{d}, \xi \in \Omega_{-p}^{R}}$ w.r.t. the topology of weak convergence of probability measures on the given Polish space $\Omega_{-p}^{R}, p>d$, is surely Gibbs, i.e., $\mu_{*} \in \mathcal{G}_{t}$. Indeed, let $\pi_{\Lambda^{(K)}}\left(d \omega \mid \xi^{(K)}\right) \stackrel{w}{\rightarrow} \mu_{*}(d \omega)$ as $\Lambda^{(K)} \nearrow \mathbb{Z}^{d}, K \rightarrow \infty$, and let us choose arbitrary $\Lambda_{0} \Subset \mathbb{Z}^{d}$ and $f \in C_{b}\left(\Omega_{-p}^{R}\right)$. Since by Lemma 3.6 also $\pi_{\Lambda_{0}} f \in C_{b}\left(\Omega_{-p}^{R}\right)$, one can directly pass to the limit $K \rightarrow \infty$ in the consistency condition $\left(\mathbf{S}_{\mathbf{i v}}\right)$ written for $\Lambda^{(K)} \supseteq \Lambda_{0}$,

$$
\int_{\Omega} \int_{\Omega} f\left(\omega^{\prime}\right) \pi_{\Lambda_{0}}\left(d \omega^{\prime} \mid \omega\right) \pi_{\Lambda^{(K)}}\left(d \omega \mid \xi^{(K)}\right)=\int_{\Omega} f(\omega) \pi_{\Lambda^{(K)}}\left(d \omega \mid \xi^{(K)}\right),
$$

and thus get the DLR equation

$$
\int_{\Omega} \int_{\Omega} f\left(\omega^{\prime}\right) \pi_{\Lambda_{0}}\left(d \omega^{\prime} \mid \omega\right) \mu_{*}(d \omega)=\int_{\Omega} f(\omega) \mu_{*}(d \omega)
$$

(iii) Analogously one can show that the subset $\left\{\mu \in \mathcal{G}_{t} \mid \mu\left(\Omega_{-p}^{R}\right)=1\right\}$ is closed in the topology of weak convergence of probability measures on $\Omega_{-p}^{R}$ with $p>d$. Since the embedding $\mathcal{C}_{-p}^{\alpha} \subseteq \Omega_{-p}^{R}$ is continuous, $\left\{\mu \in \mathcal{G}_{t} \mid \mu\left(\mathcal{C}_{-p}^{\alpha}\right)=1\right\}$ is thus closed in the topology of weak convergence on $\mathcal{C}_{-p}^{\alpha}$ with $0 \leq \alpha<1 / 2$. For the particular QLS models I, II the same result is obviously valid just in the product spaces $\left[C_{\beta}^{\alpha}\right]^{\mathbb{Z}^{d}}$ with $p>d$.

(iv) For the translation invariant systems there naturally arises the question how to construct such $\mu \in \mathcal{G}_{t}$ which are also invariant w.r.t. the discrete group of lattice translations $\mathbb{Z}_{0}^{d}$, i.e.,

$$
\mu(\Delta)=\mu\left(T_{j} \Delta\right), \text { for all } j \in \mathbb{Z}_{0}^{d}, \forall \Delta \in \mathcal{B}(\Omega),
$$

where $T_{j}$ is the shift transformation, $\left(T_{j} \omega\right)_{k}:=\omega_{k+j}, k \in \mathbb{Z}^{d}$. With this aim one standardly uses the so-called local Gibbs distributions with periodic boundary conditions (cf. e.g. AKKR02]). Consider any box $\Lambda$ of the form

$$
\Lambda:=\times_{\nu=1}^{d}\left[a_{\nu}, a_{\nu}+l_{\nu}\right] \cap \mathbb{Z}^{d} \text { with } a=\left(a_{\nu}\right)_{\nu=1}^{d} \in \mathbb{Z}^{d}, \quad l=\left(l_{\nu}\right)_{\nu=1}^{d} \in \mathbb{N}_{0}^{d},
$$

and let $\mathfrak{T}(\Lambda) \cong \mathbb{Z}^{d} / l \mathbb{Z}^{d}$ be the torus obtained by identifying its opposite walls. Respectively, we define the $\Lambda$-periodic continuation $\tilde{\omega}_{\Lambda} \in \Omega$ of the projection $\omega_{\Lambda} \in \Omega_{\Lambda}$ by $\tilde{\omega}_{\Lambda}:=\left(\omega_{j(k)}\right)_{k \in \mathbb{Z}^{d}}$, where $j(k) \in \mathfrak{T}(\Lambda)$ is the unique element such that $j(k) \cong k(\bmod l)$. Next, we can define the $\Lambda$-periodic modification of the interaction

$$
\mathcal{I}_{p e r, \Lambda}^{W}(\omega):=\sum_{M=2}^{N} \sum_{\left\{k_{1}, \ldots, k_{M}\right\} \subset \Lambda} \int_{S_{\beta}} W_{\left\{k_{1}, \ldots, k_{M}\right\}}\left(\omega_{j\left(k_{1}\right)}(\tau), \ldots, \omega_{j\left(k_{M}\right)}(\tau)\right) d \tau
$$


and the associated with it periodic local Gibbs distribution

$$
\mu_{\mathfrak{T}(\Lambda)}\left(d \omega_{T(\Lambda)}\right):=Z_{\mathfrak{T}(\Lambda)}^{-1} \exp \left\{-\mathcal{I}_{p e r, \Lambda}^{W}(\omega)\right\} \prod_{j \in \mathfrak{T}(\Lambda)} d \sigma_{\beta}\left(\omega_{j}\right)
$$

as a probability measure on $\Omega_{\mathfrak{T}(\Lambda)}:=\mathbb{R}^{\mathfrak{T}(\Lambda)}$. Suppose now that a sequence $\mu_{\mathfrak{T}\left(\Lambda^{(K)}\right)}$ converges locally weakly on $\Omega$ as $\Lambda^{(K)} \nearrow \mathbb{Z}^{d}, K \rightarrow \infty$, to some $\mu_{*} \in \mathcal{M}\left(\Omega_{t}\right)$, which means that

$$
\lim _{K \rightarrow \infty} \int_{\mathfrak{T}\left(\Lambda_{n}\right)} f(\omega) \mu_{\mathfrak{T}\left(\Lambda^{(K)}\right)}\left(d \omega_{T\left(\Lambda^{(K)}\right)}\right)=\int_{\Omega_{t}} f(\omega) \mu_{*}(d \omega)
$$

for any local $f=f\left(\mathbb{P}_{\Lambda_{0}}\right) \in C_{b}(\Omega)$. Assuming again that $W_{\left\{k_{1}, \ldots, k_{M}\right\}} \in C\left(\mathbb{R}^{M}\right)$ are local, it is easy to show that $\mu_{*} \in \mathcal{G}_{t}$. So, by construction one has the consistency relation

$$
\int_{\mathfrak{T}(\Lambda)} \int_{\Lambda_{0}} f\left(\omega^{\prime}\right) \mu_{\Lambda_{0}}\left(d \omega_{\Lambda_{0}}^{\prime} \mid \omega\right) \mu_{\mathfrak{T}\left(\Lambda^{(K)}\right)}\left(d \omega_{T(\Lambda)}\right)=\int_{\mathfrak{T}\left(\Lambda^{(K)}\right)} f(\omega) \mu_{\mathfrak{T}(\Lambda)}\left(d \omega_{\mathfrak{T}(\Lambda)}\right)
$$

in any boxes $\Lambda_{0}, \Lambda \Subset \mathbb{Z}^{d}$ such that $\Lambda^{(\rho+1)}:=\left\{k \in \mathbb{Z}^{d} \mid \operatorname{dist}\left(k, \Lambda_{0}\right) \leq \rho+1\right\} \subset \Lambda$. Since $\pi_{\Lambda_{0}} f=: g=g\left(\mathbb{P}_{\Lambda^{(K)}}\right) \in C_{b}(\Omega)$, one can directly pass in (3.71) to the limit as $\Lambda:=$ $\Lambda^{(K)} \nearrow \mathbb{Z}^{d}$ and thus get the DLR equation (3.67). Note that the required $\mathbb{Z}_{0}^{d}$-translation invariance of $\mu_{*}$ follows from the invariance of any $\mu_{\mathfrak{T}(\Lambda)}$ w.r.t. the translations of the torus $\mathfrak{T}(\Lambda)$.

\section{Flow AND integration by PARTS CharaCterization of Euclidean GibBs measure}

Analogously to what has already been done for classical lattice systems in [AKRT99, in this paper we would like to develop an alternative approach to the construction and study of Euclidean Gibbs measures for quantum lattice systems. The main ingredients of this approach are: first, the flow characterization of $\mu \in \mathcal{G}$ in terms of Radon-Nikodym derivatives w.r.t. shift transformations of the configuration space $\Omega$ and, second, the characterization (resulting from the previous one) in terms of their logarithmic derivatives via corresponding integration by parts formulas. If the interaction potentials are differentiable (as they are in our case), both characterizations are equivalent. Such alternative (to the usual through the DLR equations) characterizations of Gibbs measures have long been known for a number of specific models in statistical mechanics and field theory (see, e.g., Ro75, Ro77, HS76, Fro77, Fri82, Deu87, Ki95]). However, in rather full generality the flow description for both classical and quantum Gibbs measures was first proved in AKR97a, AKR97b. In particular, AKR97b, Sect. 4.2] contains the flow characterization for Euclidean Gibbs measures of quantum lattice systems (3.1) with harmonic pair interactions. In this section we extend the latter result to systems with many-particle interactions and give the complete characterization of $\mu \in \mathcal{G}_{t}$ in terms of their Radon-Nikodým and logarithmic derivatives. Also we observe that the local Gibbs specifications $\pi_{\Lambda}, \Lambda \Subset \mathbb{Z}^{d}$, also satisfy the same flow and integration by parts descriptions, which later will be crucial for our proof of the existence of $\mu \in \mathcal{G}_{t}$.

As an application of this alternative description of Euclidean Gibbs states, a direct analytic proof of our main Theorems I and II using the corresponding (IbP)-formulas will be presented in Subsect. 4.6 under Hypotheses $(\mathbf{H})$ and $\left(\mathbf{H}_{1 \mathrm{oc}}\right)$ (which in turn will later be proved to be satisfied, cf. Subsect. 7.3).

We assume throughout Section 4 that conditions (W), (J), (V) in Definition 3.2 always hold without any additional reference to them. 
4.1. Flow description of Euclidean Gibbs measures. We start with the flow description of $\mu \in \mathcal{G}_{t}$ in terms of its "shift"-Radon-Nikodým derivatives $a_{\theta h_{i}}, \theta \in \mathbb{R}$, along some set of admissible directions $h_{i}, i \in \mathcal{I}$, whose linear span is dense in $\Omega_{t}:=\Omega_{(s) t}^{R}$.

As already said in Subsect. 3.2.3, we consider

$$
\mathcal{H}:=l^{2} \otimes L_{\beta}^{2} \cong l^{2}\left(\mathbb{Z}^{d} \rightarrow L_{\beta}^{2}\right)
$$

with the inner product $\langle\omega, \omega\rangle_{\mathcal{H}}:=\|\omega\|_{\mathcal{H}}^{2}$ as a tangent Hilbert space to $\Omega$. For the remainder of the paper, we fix the concrete orthonormal basis in $\mathcal{H}$ (for shorthand, we denote it by $\left.\operatorname{bas}(\mathcal{H}):=\left\{h_{i}\right\}_{i \in \mathbb{Z}^{d+1}}\right)$ consisting of the vectors

$$
h_{i}:=e_{k} \otimes \varphi_{n}=\left(\delta_{k-k^{\prime}} \varphi_{n}\right)_{k^{\prime} \in \mathbb{Z}^{d}}, \quad i=(k, n) \in \mathbb{Z}^{d+1}, k \in \mathbb{Z}^{d}, n \in \mathbb{Z} .
$$

Here $e_{k}:=\left(\delta_{k-k^{\prime}}\right)_{k^{\prime} \in \mathbb{Z}^{d}}$, and $\varphi_{n} \in C_{\beta}^{\infty}$ are the eigenvectors of the operator $A$ in $H:=L_{\beta}^{2}$ given by $(3.24)$.

For $i=(k, n) \in \mathbb{Z}^{d+1}$ and $\theta \in \mathbb{R}$, we define the corresponding relative many-particle interaction by

$$
\begin{array}{r}
\mathcal{I}_{r e l}^{W}\left(\omega \mid \theta h_{i}\right):=\sum_{M=2}^{N} \sum_{\substack{k_{1}:=k \\
\left\{k_{1}, \ldots, k_{M}\right\} \subset \mathbb{Z}^{d}}} \int_{S_{\beta}}\left[W_{\left\{k_{1}, \ldots, k_{M}\right\}}\left(\omega_{k_{1}}+\theta \varphi_{n}, \omega_{k_{2}}, \ldots, \omega_{k_{M}}\right)\right. \\
\left.-W_{\left\{k_{1}, \ldots, k_{M}\right\}}\left(\omega_{k_{1}}, \ldots, \omega_{k_{m}}\right)\right] d \tau .
\end{array}
$$

(In the notation of (3.41) it heuristically equals $\mathcal{I}_{\mathbb{Z}^{d}}^{W}\left(\omega+\theta h_{i}\right)-\mathcal{I}_{\mathbb{Z}^{d}}^{W}(\omega)$.) Since $\left(\mathbf{W}_{\mathbf{i}}\right)$ and $(\mathbf{J})$ hold, arguments similar to those applied in the proof of Lemmas 3.5 and 3.6 yield the following properties of the function $(\theta, \omega) \mapsto \mathcal{I}_{\text {rel }}^{W}\left(\omega \mid \theta h_{i}\right)$ for every $i \in \mathbb{Z}^{d}$ :

The sum in (4.2) with the integrands replaced by their absolute values

$$
\begin{aligned}
& \text { converges for all } \theta \in \mathbb{R}, \omega \in \mathcal{L}_{-p}^{R} \text {, and }(\theta, \omega) \mapsto \mathcal{I}_{r e l}^{W}\left(\omega \mid \theta h_{i}\right) \\
& \text { is uniformly Lipschitz continuous on balls in } \mathbb{R} \times \mathcal{L}_{-p}^{R}, p>d \text {. }
\end{aligned}
$$

Moreover, exploiting $\left(\mathbf{W}_{\mathbf{i i}}\right)$ and using a standard theorem of analysis on the differentiability of uniformly convergent series (cf. e.g. Ca67, Theorem 3.6.2]), one can show that

$$
\begin{aligned}
& \frac{\partial}{\partial \theta} \mathcal{I}_{\text {rel }}^{W}\left(\omega \mid \theta h_{i}\right) \\
& \quad=\sum_{M=2}^{N} \sum_{\substack{k_{1}:=k \\
\left\{k_{1}, \ldots, k_{M}\right\} \subset \mathbb{Z}^{d}}} \int_{S_{\beta}}\left[\partial_{1} W_{\left\{k_{1}, \ldots, k_{M}\right\}}\left(\omega_{k_{1}}+\theta \varphi_{n}, \omega_{k_{2}}, \ldots, \omega_{k_{M}}\right) \cdot \varphi_{n}\right](\tau) d \tau
\end{aligned}
$$

exists with the same regularity properties of the function $(\theta, \omega) \longmapsto \frac{\partial}{\partial \theta} \mathcal{I}_{r e l}^{W}\left(\omega \mid \theta h_{i}\right)$ as described in (4.3).

Next, let us define the following densities $a_{\theta h_{i}}: \Omega_{t} \rightarrow \mathbb{R}$, where (cf. (3.35) and (4.2))

$$
a_{\theta h_{i}}:=a_{\theta h_{i}}^{A_{\beta}} \cdot a_{\theta h_{i}}^{V} \cdot a_{\theta h_{i}}^{W}
$$

and

$$
a_{\theta h_{i}}^{A_{\beta}}(\omega):=a_{\theta \varphi_{n}}^{A_{\beta}}\left(\omega_{k}\right), \quad a_{\theta h_{i}}^{V}(\omega):=a_{\theta \varphi_{n}}^{V_{k}}\left(\omega_{k}\right), \quad a_{\theta h_{i}}^{W}(\omega):=\exp \left\{-\mathcal{I}_{r e l}^{W}\left(\omega \mid \theta h_{i}\right)\right\} .
$$

According to the discussion above,

$$
\begin{aligned}
(\theta, \omega) \longmapsto & a_{\theta h_{i}}^{W}(\omega), \\
& \frac{\partial}{\partial \theta} a_{\theta h_{i}}^{W}(\omega) \text { are uniformly Lipschitz continuous } \\
& \text { on balls in } \mathbb{R} \times \mathcal{L}_{-p}^{R}, p>d .
\end{aligned}
$$


Thus, combining (3.37) and (4.6), we get the important, for later use, assertion which we formulate as follows:

Lemma 4.1. For every $i=(k, n) \in \mathbb{Z}^{d+1}$ the functions

$$
(\theta, \omega) \longmapsto a_{\theta h_{i}}(\omega), \frac{\partial}{\partial \theta} a_{\theta h_{i}}(\omega)
$$

are uniformly Lipschitz continuous on balls in $\mathbb{R} \times \Omega_{-p ; k}^{R}, p>d$.

The main result of this subsection is Proposition 4.2, which extends the corresponding result of Theorem 4.6 in AKR97b, where (for simplicity only) the case of harmonic pair interactions (i.e., the particular QLS model (1.2)) was treated. The method of the proof proposed in that paper (see also the earlier papers Iw85, Fu91, Ro75, Fri82] on related topics) also applies in our situation with some technical alterations. However, in order to keep the exposition self-contained, here we repeat the key steps of [AKR97b] and present the complete proof.

Proposition 4.2 (Flow Description of Tempered Gibbs States). Let $\mathcal{M}_{t}^{a}$ denote the set of all probability measures $\mu$ on $(\Omega, \mathcal{B}(\Omega))$ which satisfy the temperedness condition (3.64) and are quasi-invariant w.r.t. the shifts $\omega \longmapsto \omega+\theta h_{i}, \theta \in \mathbb{R}, i=(k, n) \in \mathbb{Z}^{d+1}$, with the Radon-Nikodým derivatives

$$
\frac{d \mu\left(\omega+\theta h_{i}\right)}{d \mu(\omega)}:=a_{\theta h_{i}}(\omega)
$$

Then $\mathcal{G}_{t}=\mathcal{M}_{t}^{a}$.

Proof. Define $\mathcal{T}:=\left[T_{\beta}\right]^{\mathbb{Z}_{0}^{d}}:=\operatorname{lin}\left\{h_{i}\right\}_{i \in \mathcal{I}} \subset \Omega$ as a subspace of all finite sequences $\eta=$ $\left(\eta_{k}\right)_{k \in \mathbb{Z}^{d}}\left(\eta_{k}=0\right.$ when $\left.|k|>K(\eta)>0\right)$ with components $\eta_{k} \in T_{\beta}:=\operatorname{lin}\left\{\varphi_{n}\right\}_{n \in \mathbb{Z}}$. By the standard group argument, $\mathcal{M}_{t}^{a}$ is exactly the set of all $\mu \in \mathcal{M}_{t}$ which are $\mathcal{T}$-quasiinvariant with the cocycle

$$
\frac{d \mu(\omega+\eta)}{d \mu(\omega)}:=a_{\eta}(\omega)=a_{\eta}^{A_{\beta}}(\omega) \cdot a_{\eta}^{V}(\omega) \cdot a_{\eta}^{W}(\omega)>0, \quad \eta \in \mathcal{T}, \omega \in \Omega_{t} .
$$

Here

$$
\begin{aligned}
& a_{\eta}^{A_{\beta}}(\omega):=\exp \left\{-\sum_{k \in \mathbb{Z}^{d}:|k| \leq K(\eta)}\left[\left(A_{\beta} \eta_{k}, \omega_{k}\right)_{H}+\frac{1}{2}\left(A_{\beta} \eta_{k}, \eta_{k}\right)_{H}\right]\right\} \\
& a_{\eta}^{V}(\omega):=\exp \left\{-\sum_{k \in \mathbb{Z}^{d}:|k| \leq K(\eta)} \int_{S_{\beta}}\left[V_{k}\left(\omega_{k}+\eta_{k}\right)-V_{k}\left(\omega_{k}\right)\right] d \tau\right\} \\
& a_{\eta}^{W}(\omega):=\exp \left\{-\mathcal{I}_{r e l}^{W}(\omega \mid \eta)\right\}
\end{aligned}
$$

and

$$
\begin{array}{r}
\mathcal{I}_{r e l}^{W}(\omega \mid \eta):=\sum_{M=2}^{N} \sum_{\substack{\left\{k_{1}, \ldots, k_{M}\right\} \subset \mathbb{Z}^{d} \\
\exists 1 \leq L \leq M:\left|k_{L}\right| \leq K(\eta)}} \int_{S_{\beta}}\left[W_{\left\{k_{1}, \ldots, k_{M}\right\}}\left(\omega_{k_{1}}+\eta_{k_{1}}, \ldots, \omega_{k_{M}}+\eta_{k_{M}}\right)\right. \\
\left.-W_{\left\{k_{1}, \ldots, k_{M}\right\}}\left(\omega_{k_{1}}, \ldots, \omega_{k_{M}}\right)\right] d \tau .
\end{array}
$$

As follows from the discussion preceding Lemma 4.1, the above definitions are correct, and for all $k \in \mathbb{Z}^{d}, \eta \in \mathcal{T}$ the functions

$$
\mathbb{R} \times \Omega_{t} \ni(\theta, \omega) \longmapsto a_{\theta \eta}(\omega) \text { are continuous and locally bounded. }
$$

(i) $\mathcal{G}_{t} \subseteq \mathcal{M}_{t}^{a}$ : This inclusion is obvious. Suppose that $\mu \in \mathcal{G}_{t}$, i.e., it satisfies the DLR equations (3.59). Then from the quasi-invariance property of the probability kernels 
$\pi_{\Lambda}(d \omega \mid \xi)$ (see also Subsect. 5.1 below), one has that for any $k \in \Lambda \Subset \mathbb{Z}^{d}, \eta:=\theta h_{i} \in \mathcal{T}$ and $\Delta \in \mathcal{B}(\Omega)$,

$$
\begin{aligned}
\int_{\Omega_{t}} \mathbf{1}_{\Delta}(\omega+\eta) \mu(d \omega) & =\int_{\Omega_{t}} \int_{\Omega_{t}} \mathbf{1}_{\Delta}(\omega+\eta) \pi_{\Lambda}(d \omega \mid \xi) \mu(d \xi) \\
& =\int_{\Omega_{t}} \int_{\Omega_{t}} \mathbf{1}_{\Delta}(\omega) a_{\eta}(\omega) \pi_{\Lambda}(d \omega \mid \xi) \mu(d \xi)=\int_{\Omega_{t}} \mathbf{1}_{\Delta}(\omega) a_{\eta}(\omega) \mu(d \omega) .
\end{aligned}
$$

This relation exactly means that $\mu \in \mathcal{M}_{a}^{t}$.

(ii) $\mathcal{M}_{t}^{a} \subseteq \mathcal{G}_{t}$ : Keeping the notation of Subsect. 3.3, for every $k \in \mathbb{Z}^{d}$ define $\Lambda_{k}:=\{k\}$, $\Lambda_{k}^{c}:=\mathbb{Z}^{d} \backslash\{k\}$ and $\omega_{\Lambda_{k}^{c}}:=\mathbb{P}_{\Lambda_{k}^{c}} \omega \in \Omega_{\Lambda_{k}^{c}}$. Now we start with arbitrary $\mu \in \mathcal{M}_{t}^{a}$ and disintegrate this measure w.r.t. its projection $\mu_{\Lambda_{k}^{c}}:=\mu \mathbb{P}_{\Lambda_{k}^{c}}^{-1}$ onto $\Omega_{\Lambda_{k}^{c}}$ (see, e.g., $\mathrm{Pa} 67$, p. 147, Theorem 8.1]):

$$
\mu\left(d \omega_{k}, d \omega_{\Lambda_{k}^{c}}\right)=\nu_{\omega_{\Lambda_{k}^{c}}}\left(d \omega_{k}\right) \mu_{\Lambda_{k}^{c}}\left(d \omega_{\Lambda_{k}^{c}}\right)
$$

Here $\nu_{\omega_{\Lambda_{k}}}\left(d \omega_{k}\right)$ are some probability measures (= regular conditional distributions given $\left.\omega_{\Lambda_{k}^{c}}\right)$ on $\left(C_{\beta}, \mathcal{B}\left(C_{\beta}\right)\right)$. By the temperedness condition on $\mu$, it is clear that $\mu_{\Lambda_{k}^{c}}\left(\Omega_{\Lambda_{k}^{c}, t}\right)=1$, where $\Omega_{\Lambda_{k}^{c}, t}:=\mathbb{P}_{\Lambda_{k}^{c}} \Omega_{t}$. On the other hand, from (4.8) and (4.12) one can straightforwardly verify (using crucially the continuity property (4.11) of $a_{\eta_{k}}(\omega)$; cf. Ro75, Proposition 3]) the quasi-invariance of the measures $\nu_{\omega_{\Lambda_{k}}}$ in the following sense: there exists a Borel subset $\Delta_{k} \subseteq \Omega_{\Lambda_{k}^{c}, t}$ of full measure $\mu_{\Lambda_{k}^{c}}$ (i.e., $\mu_{\Lambda_{k}^{c}}\left(\Delta_{k}\right)=1$ ), such that for any $\omega_{\Lambda_{k}^{c}} \in \Delta_{k}$ and $\eta_{k} \in T_{\beta}$,

$$
\frac{d \nu_{\omega_{\Lambda_{k}^{c}}}\left(\omega_{k}+\eta_{k}\right)}{d \nu_{\omega_{\Lambda_{k}^{c}}}\left(d \omega_{k}\right)}=a_{\eta_{k}}\left(\omega_{k}, \omega_{\Lambda_{k}^{c}}\right), \quad \omega_{k} \in C_{\beta}\left(\bmod \nu_{\omega_{\Lambda_{k}^{c}}}\right) .
$$

From now on fix any $\xi_{\Lambda_{k}^{c}} \in \Delta_{k}$, and let us show that (4.13) implies

$$
\nu_{\xi_{\Lambda_{k}^{c}}}\left(d \omega_{k}\right)=\mu_{\Lambda_{k}}\left(d \omega_{k} \mid \xi_{\Lambda_{k}^{c}}\right)
$$

where $\mu_{\Lambda_{k}}\left(d \omega_{k} \mid \xi_{\Lambda_{k}^{c}}\right)$ is the Gibbs measure in the volume $\Lambda_{k}$ with the boundary condition $\xi_{\Lambda_{k}^{c}}:=\omega_{\Lambda_{k}^{c}}$. We recall that according to definition (3.42),

$$
\begin{aligned}
& \mu_{\Lambda_{k}}\left(d \omega_{k} \mid \xi_{\Lambda_{k}^{c}}\right):= Z_{\Lambda_{k}}^{-1}(\xi) \cdot \exp \left\{-\int_{S_{\beta}} V_{k}\left(\omega_{k}\right) d \tau\right\} \\
& \times \exp \left\{\sum_{\substack{\left\{k_{1}, \ldots, k_{M}\right\} \subset \mathbb{Z}^{d} \\
k_{1}:=k ; 2 \leq M \leq N}} \int_{S_{\beta}} W_{\left\{k_{1} \ldots, k_{M}\right\}}\left(\omega_{k_{1}}, \xi_{k_{2}}, \ldots, \xi_{k_{M}}\right) d \tau\right\} \gamma_{\beta}\left(d \omega_{k}\right),
\end{aligned}
$$

where $\gamma_{\beta}$ is the Gaussian measure with correlation operator $A_{\beta}^{-1}$. But $\nu\left(d \omega_{k}\right):=$ $\mu_{\Lambda_{k}}\left(d \omega_{k} \mid \xi_{\Lambda_{k}^{c}}\right)$ is the unique probability measure on $\left(C_{\beta}, \mathcal{B}\left(C_{\beta}\right)\right)$ which satisfies the flow description (4.13), i.e.,

$$
\frac{d \nu\left(\omega_{k}+\eta_{k}\right)}{d \nu\left(\omega_{k}\right)}=a_{\eta_{k}}\left(\omega_{k}\right), \quad \omega_{k} \in C_{\beta}(\bmod \nu)
$$


for all $\eta_{k} \in T_{\beta}$ with a cocycle having the explicit form

$$
\begin{aligned}
& a_{\eta_{k}}\left(\omega_{k}\right):= \exp \left\{-\left(A_{\beta} \eta_{k}, \omega_{k}\right)_{H}-\frac{1}{2}\left(A_{\beta} \eta_{k}, \eta_{k}\right)_{H}-\int_{S_{\beta}}\left[V_{k}\left(\omega_{k}+\eta_{k}\right)-V_{k}\left(\omega_{k}\right)\right] d \tau\right\} \\
& \times \exp \left\{-\sum_{\substack{\left\{k_{1}, \ldots, k_{M}\right\} \subset \mathbb{Z}^{d} \\
k_{1}:=k ; 2 \leq M \leq N \\
-}} \int_{S_{\beta}}\left[W_{\left\{k_{1}, \ldots, k_{M}\right\}}\left(\omega_{k_{1}}+\eta_{k_{1}}, \xi_{k_{2}}, \ldots, \xi_{k_{M}}\right)\right.\right. \\
&\left.\left.-W_{\left\{k_{1}^{\prime} \ldots, k_{M}\right\}}\left(\omega_{k_{1}}, \xi_{k_{2}}, \ldots, \xi_{k_{M}}\right)\right] d \tau\right\} .
\end{aligned}
$$

To check this, let us introduce the new measure

$$
\sigma\left(d \omega_{k}\right):=\exp \left\{\int_{S_{\beta}}\left[V_{k}\left(\omega_{k}\right)+\sum_{\substack{\left\{k_{1}, \ldots, k_{M}\right\} \subset \mathbb{Z}^{d} \\ k_{1}:=k ; 2 \leq M \leq N}} W_{\left\{k_{1}, \ldots, k_{M}\right\}}\left(\omega_{k_{1}}, \xi_{k_{2}}, \ldots, \xi_{k_{M}}\right)\right] d \tau\right\} \nu\left(d \omega_{k}\right),
$$

which (due to our assumptions on the potentials $V$ and $W$ ) is at least $\sigma$-finite on $\left(C_{\beta}, \mathcal{B}\left(C_{\beta}\right)\right)$. By (4.16)-(4.18) we have that

$$
\frac{d \sigma\left(\omega_{k}+\eta_{k}\right)}{d \sigma\left(\omega_{k}\right)}=\exp \left\{-\left(A_{\beta} \eta_{k}, \omega_{k}\right)_{H}-\frac{1}{2}\left(A_{\beta} \eta_{k}, \eta_{k}\right)_{H}\right\}
$$

or, equivalently,

$$
\frac{d \sigma\left(\omega_{k}+\eta_{k}\right)}{d \sigma\left(\omega_{k}\right)}=\frac{d \gamma_{\beta}\left(\omega_{k}+\eta_{k}\right)}{d \gamma_{\beta}\left(\omega_{k}\right)}
$$

We claim that (4.20) implies

$$
\sigma\left(C_{\beta}\right)<\infty \text { and } \frac{\sigma\left(d \omega_{k}\right)}{\sigma\left(C_{\beta}\right)}=\gamma_{\beta}\left(d \omega_{k}\right)
$$

To this end we exactly repeat the corresponding arguments from the proof of Theorem 4.6 in AKR97b. Let $A_{\beta} \varphi_{n}=\lambda_{n} \varphi_{n}$ with any $n \in \mathbb{Z} \backslash\{0\}$, and let us consider the image measure $\sigma_{n}$ of $\sigma$ under the mapping $\omega_{k} \longmapsto\left(\omega_{k}, \varphi_{n}\right)_{H}$. Then, by (4.20) and the product structure of the right-hand side of (4.19), $\sigma_{n}$ is quasi-invariant with "shift"-RadonNikodým derivatives equal to that of the Gaussian measure $\sqrt{\frac{\lambda_{n}}{2 \pi}} \exp \left(-\frac{1}{2} \lambda_{n} q^{2}\right) d q:=$ $\gamma_{n}(q) d q$ on $\mathbb{R}$. Herewith it is well known and could be easily verified that $\sigma_{n}(d q) \sim d q$. Hence, if $\rho:=d \sigma_{n} / d q$, then for all $\theta \in \mathbb{R}$,

$$
\frac{\rho(q+\theta)}{\gamma_{n}(q+\theta)}=\frac{\rho(q)}{\gamma_{n}(q)}, \quad q \in \mathbb{R}(\bmod d q)
$$

The latter means that $\rho / \gamma_{n} \equiv$ const $(\bmod d q)$. Thus $\sigma_{n}(d q)=$ const $\times \gamma_{n}(q) d q$ and, in particular, $\sigma\left(C_{\beta}\right)=\sigma_{n}(\mathbb{R})<\infty$. Since $\sigma$ is finite and satisfies (6.22), it readily follows from [Ro75, Proposition 4] that $\sigma\left(d \omega_{k}\right)=\sigma\left(C_{\beta}\right) \cdot \gamma_{\beta}\left(d \omega_{k}\right)$.

Consequently, combining (4.15) and (4.18), we deduce that $\nu\left(d \omega_{k}\right)=$ const $\cdot \nu_{\xi_{\Lambda_{k}^{c}}}\left(d \omega_{k}\right)$ and, since both are probability measures, they coincide. Thus (4.14) is shown. Herewith, noting that each measure from $\mathcal{G}_{t}$ is fully determined by $\left\{\pi_{\Lambda_{k}}\right\}_{k \in \mathbb{Z}^{d}}$ (cf. Remark 3.10 (i)), we get the desired inclusion $\mu \in \mathcal{G}_{t}$.

Remark 4.3. Actually, the flow characterization in Proposition 4.2 is true under minimal assumptions on the potentials, which guarantee (besides the well-definedness of the local specification $\pi_{\Lambda}$ ) merely the continuity and local boundedness of the functions $\mathbb{R} \times \Omega_{t} \ni$ 
$(\theta, \omega) \longmapsto a_{\theta h_{i}}(\omega) \in \mathbb{R}$ for all $i \in \mathcal{I}$. For the quantum systems with local interaction such as the particular models (2.2) and (2.23) discussed in Sect. 2, the Radon-Nikodým derivatives $a_{\theta h_{i}}(\omega)$ given by (4.8) are well defined for all $\omega \in \Omega$, and hence the flow description (4.7) is valid for all Gibbs measures $\mu \in \mathcal{G}$.

4.2. Smooth functions on $\Omega$. In applications, however, it is more convenient to use not the flow characterization itself, but its infinitesimal form, which we proceed to describe in the following subsections.

By Proposition 4.2, for any $\mu \in \mathcal{G}_{t}$ and all bounded measurable functions $f: \Omega \rightarrow \mathbb{R}$,

$$
\int_{\Omega} f(\omega) a_{\theta h_{i}}(\omega) d \mu(\omega)=\int_{\Omega} f\left(\omega-\theta h_{i}\right) d \mu(\omega)
$$

and thus

$$
\lim _{\theta \rightarrow \pm 0} \int_{\Omega} f(\omega) \frac{a_{\theta h_{i}}(\omega)-1}{\theta} d \mu(\omega)=-\lim _{\theta \rightarrow \mp 0} \int_{\Omega} \frac{f\left(\omega+\theta h_{i}\right)-f(\omega)}{\theta} d \mu(\omega)
$$

provided the above limits exist. Henceforth, we should first introduce some spaces of differentiable functions on the loop lattice $\Omega$ needed to specify the meaning of (4.23) and the (IbP)-formulas resulting from it. Actually, Subsects. 4.2.1-4.2.4 can be viewed as a collection of standard definitions and facts from convex analysis, which we modify for our concrete situation.

4.2.1. Partially differentiable functions. Let $X, Y$ be locally convex spaces and let $\Phi$ : $X \rightarrow Y$. We recall that the partial derivatives on the right, resp. left, in the direction $h \in X$ of the function $\Phi$ at a point $x \in X$ are defined by

$$
\partial_{h}^{+} \Phi(x):=\lim _{\theta \rightarrow+0} \frac{\Phi(x+\theta h)-\Phi(x)}{\theta}, \quad \partial_{h}^{-} \Phi(x):=\lim _{\theta \rightarrow-0} \frac{\Phi(x+\theta h)-\Phi(x)}{\theta} .
$$

If the right and left limits in (4.24) coincide, one says that there exists the corresponding partial derivative $\partial_{h} \Phi(x)$ in the direction $h$. For $m \in \mathbb{N}$ and given vectors $h_{1}, \ldots, h_{m} \in$ $X$, we denote by $C^{m}\left(X \rightarrow Y ; h_{1}, \ldots, h_{m}\right)$ the set of all functions $\Phi \in C(X \rightarrow Y)$ having continuous partial derivatives $\partial_{h_{m}} \ldots \partial_{h_{1}} \Phi: X \rightarrow Y$. Respectively, by $C^{m}(X \rightarrow$ $Y), m \in \mathbb{N} \cup\{\infty\}$, we shall denote the set of all $\Phi \in C(X \rightarrow Y)$ having continuous partial derivatives $\partial_{h_{l}} \ldots \partial_{h_{1}} \Phi: X \rightarrow Y$ of any order $0 \leq l \leq m$ along arbitrary vectors $h_{1}, \ldots, h_{l} \in X$. Then, as usual,

$$
C_{b, l o c}^{m}(X \rightarrow Y) \supseteq C_{b}^{m}(X \rightarrow Y) \supseteq C_{0}^{m}(X \rightarrow Y)
$$

will mean the subspaces of those $\Phi \in C^{m}(X \rightarrow Y)$ which satisfy the extra assumptions of local, resp. global, boundedness of all their partial derivatives, or, in addition, of boundedness of their support $(\operatorname{supp} \Phi:=\{x \in X \mid \Phi(x) \neq 0\})$. For short, we shall omit $Y:=\mathbb{R}$ in the corresponding notation and write, e.g., $C^{m}(X):=C^{m}(X \rightarrow \mathbb{R})$. It should be stressed that such a type of directional differentiability (actually, even in a weaker sense, along some total set of $h \in X$ ) will be quite enough for our applications, and thus we do not discuss here the more involved notions of Gâteaux or Fréchet derivatives. Starting from Subsect. 3.2.3 we will mainly use the function spaces $C^{m}\left(\Omega_{-p}^{R}\right) \supset C^{m}\left(\Omega_{-p ; k}^{R}\right)$, $k \in \mathbb{Z}^{d}$ (and their subspaces as described above) with the underlying space $X$ being in this case $\left(\Omega_{-p}^{R}, \rho_{-p, R}\right)$, resp. $\left(\Omega_{-p, k}^{R},\|\cdot\|_{-p, R ; k}\right)$.

4.2.2. The norm function on $L_{\beta}^{R}$ and $C_{\beta}$. It is well known that the norm function $x \rightarrow$ $\Phi(x):=|x|_{X}$ on an arbitrary Banach space $X$ may be not differentiable. But as follows by a simple convexity argument applied to (4.24), there always exist the partial derivatives $\partial_{h}^{+}|x|_{X}$, resp. $\partial_{h}^{-}|x|_{X}$, on the right, resp. on the left, at every point $x \in X$ and along 
every vector $h \in X$. Since $\partial_{h}^{+}|x|_{X}$ and $\partial_{h}^{-}|x|_{X}$ do not necessarily coincide, one introduces the subdifferential $\partial|\cdot|_{X}: X \rightarrow X^{*}$ as the (possibly multivalued) mapping

$$
\partial|x|_{X}:=\left\{\left.x^{*} \in X^{*}|| x^{*}\right|_{X^{*}}=1,\left(x, x^{*}\right)=|x|_{X}\right\}, \quad \forall x \in X,
$$

where $(\cdot, \cdot)$ denotes the canonical pairing between $X$ and its dual $X^{*}$. Then for all $x, h \in$ $X$,

$$
-|h|_{X} \leq \partial_{h}^{-}|x|_{X}=\min _{x^{*} \in \partial|x|_{X}}\left(x, x^{*}\right) \leq \max _{x^{*} \in \partial|x|_{X}}\left(x, x^{*}\right)=\partial_{h}^{+}|x|_{X} \leq|h|_{X} .
$$

Besides, as is easy to see from the definition (4.24), for fixed $h \in X$, the corresponding derivatives $\partial_{h}^{+}|x|_{X}$, resp. $\partial_{h}^{-}|x|_{X}$, are semicontinuous (above, resp. below) functions of $x \in X$.

The following two examples will be of special importance for us. Consider the norm function in the spaces $X:=L_{\beta}^{R}, 1<R<\infty$. It is (Fréchet) differentiable if $x \neq 0$, which means that $\partial_{h}^{+}|x|_{L_{\beta}^{R}}=\partial_{h}^{-}|x|_{L_{\beta}^{R}}$ and $\partial|x|_{L_{\beta}^{R}}$ consists of the unique $x^{*}:=|x|_{L_{\beta}^{R}}^{1-R}|x|^{R-2} x \in$ $L_{\beta}^{R^{\prime}}, R^{\prime}=R(R-1)^{-1}$. But this is not the case for $X:=C_{\beta}$, for which it is well known that the norm function $|\cdot|_{C_{\beta}}$ is not (Gâteaux) differentiable everywhere on $C_{\beta} \backslash\{0\}$. Indeed,

$$
\begin{gathered}
\exists \partial_{h}|x|_{C_{\beta}}=\partial_{h}^{ \pm}|x|_{C_{\beta}} \text { for } x, h \in C_{\beta} \text { if and only if } \\
h(\tau)= \pm h\left(\tau^{\prime}\right) \text { for all } \tau, \tau^{\prime} \in S_{\beta} \text { such that } x(\tau)= \pm x\left(\tau^{\prime}\right)=|x|_{C_{\beta}} .
\end{gathered}
$$

Thus in the relevant calculations we will use either the right or left derivatives $\partial_{h}^{+}|x|_{C_{\beta}}$, resp. $\partial_{h}^{-}|x|_{C_{\beta}}$, and estimate them through the inequality (4.26). For more information on related topics in convex analysis we refer, e.g., to [Dei85].

4.2.3. Cylinder functions on $\Omega$. Since configurations of the system are described by sequences of continuous loops over $\mathbb{Z}^{d}$, there follow a few natural notions of cylinder functions w.r.t. the single spin space and the lattice structure:

(i) (Cylindricity w.r.t. the lattice basis $\left.\left\{e_{k}\right\}_{k \in \mathbb{Z}^{d}}\right)$ By $\mathcal{F} C^{m}\left(\Omega ; \mathbb{Z}^{d}\right), m \in \mathbb{N} \cup\{0,+\infty\}$, we shall denote the set of all local functions $f: \Omega \rightarrow \mathbb{R}$ which can be represented as $f(\omega)=f_{\Lambda}\left(\mathbb{P}_{\Lambda} \omega\right)$ with some $f_{\Lambda} \in C^{m}\left(\Omega_{\Lambda}\right)$ and $\Lambda \Subset \mathbb{Z}^{d}$.

(ii) (Cylindricity w.r.t. the basis $\left\{h_{i}\right\}_{i \in \mathbb{Z}^{d+1}}$ in $\left.\mathcal{H}\right) \operatorname{By} \mathcal{F} C^{m}(\Omega ; \mathcal{H})\left(\subset \mathcal{F} C^{m}\left(\Omega ; \mathbb{Z}^{d}\right)\right)$ we shall respectively denote the set of all functions $f: \Omega \rightarrow \mathbb{R}$ of the form

$$
f(\omega)=f_{L}\left(\left\langle\omega, h_{i_{1}}\right\rangle_{\mathcal{H}}, \ldots,\left\langle\omega, h_{i_{L}}\right\rangle_{\mathcal{H}}\right),
$$

with some $L \in \mathbb{N}, f_{L} \in C^{l}\left(\mathbb{R}^{L}\right)$ and $i_{1}, \ldots, i_{L} \in \mathbb{Z}^{d+1}$. Then for the corresponding partial derivatives in the directions $h \in \Omega$ we have

$$
\partial_{h} f(\omega)=\sum_{l=1}^{L} \partial_{l} f_{L}\left(\left\langle\omega, h_{i_{1}}\right\rangle_{\mathcal{H}}, \ldots,\left\langle\omega, h_{i_{L}}\right\rangle_{\mathcal{H}}\right)\left\langle h, h_{i_{l}}\right\rangle_{\mathcal{H}}
$$

Replacing $C^{m}\left(\mathbb{R}^{L}\right)$ in (4.28) by $C_{b}^{m}\left(\mathbb{R}^{L}\right)$ (resp. $C_{b, l o c}^{m}\left(\mathbb{R}^{L}\right)$ ), we obtain the subsets $\mathcal{F} C_{b}^{m}(\Omega ; \mathcal{H})\left(\right.$ resp. $\left.\mathcal{F} C_{b, l o c}^{m}(\Omega ; \mathcal{H})\right)$.

(iii) (Space-time cylindricity) Note that for any fixed $k \in \mathbb{Z}^{d}$ and $\tau \in S_{\beta}$,

$$
\left\langle\omega, \delta_{(k, \tau)}\right\rangle_{\mathcal{H}}:=\omega_{k}(\tau), \quad \omega=\left(\omega_{k}\right)_{k \in \mathbb{Z}^{d}} \in \Omega,
$$

is a well-defined bounded linear functional $\delta_{(k, \tau)} \in \Omega^{*}$. Hence, we can introduce the set $\mathcal{F} C^{m}\left(\Omega ; \mathbb{Z}^{d} \times S_{\beta}\right)$ (and also its subsets $\left.\mathcal{F} C_{b}^{m}\left(\Omega ; \mathbb{Z}^{d} \times S_{\beta}\right), \mathcal{F} C_{b, l o c}^{m}\left(\Omega ; \mathbb{Z}^{d} \times S_{\beta}\right)\right)$ consisting of all cylinder (w.r.t. the family $\left\{\delta_{(k, \tau)}\right\}_{(k, \tau) \in \mathbb{Z}^{d} \times S_{\beta}} \subset \Omega^{*}$ ) functions $f: \Omega \rightarrow \mathbb{R}$ of the form

$$
f(\omega)=f_{L}\left(\omega_{k_{1}}\left(\tau_{1}\right), \ldots, \omega_{k_{L}}\left(\tau_{L}\right)\right)=f_{L}\left(\left\langle\omega, \delta_{\left(k_{1}, \tau_{1}\right)}\right\rangle_{\mathcal{H}}, \ldots,\left\langle\omega, \delta_{\left(k_{L}, \tau_{L}\right)}\right\rangle_{\mathcal{H}}\right)
$$


where $L \in \mathbb{N}, f_{L} \in C^{m}\left(\mathbb{R}^{L}\right)$ and $\left(k_{1}, \tau_{1}\right), \ldots,\left(k_{L}, \tau_{L}\right) \in \mathbb{Z}^{d} \times S_{\beta}$. As can be checked by direct calculation, for any $h \in \Omega$,

$$
\partial_{h} f(\omega)=\sum_{l=1}^{L} \partial_{l} f_{L}\left(\omega_{k_{1}}\left(\tau_{1}\right), \ldots, \omega_{k_{L}}\left(\tau_{L}\right)\right) \cdot h_{k_{l}}\left(\tau_{l}\right)
$$

exists. Obviously, $\mathcal{F} C^{m}(\Omega ; \mathcal{H}), \mathcal{F} C^{m}\left(\Omega ; \mathbb{Z}^{d} \times S_{\beta}\right) \subset C^{m}\left(\Omega_{-p}^{R}\right)$ for all $p \geq 0, R \geq 1$.

4.2.4. Approximations by smooth functions. Since we are dealing with functions on infinite-dimensional vector spaces (by the way, in our case $\mathcal{F} C_{b}(\Omega ; \mathcal{H}) \cap C_{0}\left(\Omega_{-p ; k}^{-R}\right)=\emptyset$ and the norms $\|\cdot\|_{-p, R ; k}$ defining the topology in $\Omega_{-p}^{R}$ are not differentiable), a possible approximation of $f: \Omega \rightarrow \mathbb{R}$ by smooth, resp. boundedly supported, functions should be treated carefully. For the sake of completeness, here we collect several technical assertions (more or less of common knowledge and even true in a more general setting on locally convex spaces), which will be used below when extending the (IbP)-formula (4.39) to suitable classes of differentiable functions on $\Omega$.

Lemma 4.4. Let $\mu$ be a Borel measure on $\Omega$ such that $\mu\left(\Omega_{-p}^{R}\right)=1$ for some $p>d$, $R \geq 2$. Then the following assertions hold:

(i) $\left(C^{1}\right.$-Approximation by Smooth Cylinder Functions) Given any $h_{i} \in \operatorname{bas}(\mathcal{H})$ and $f \in C_{b}^{1}\left(\Omega_{-p}^{R} ; h_{i}\right)$, in each of the spaces $\mathcal{F} C^{\infty}(\Omega ; \mathcal{H})$ and $\mathcal{F} C^{\infty}\left(\Omega ; \mathbb{Z}^{d} \times S_{\beta}\right)$ there exist corresponding sequences $\left\{f^{(K)}\right\}_{K \in \mathbb{N}}$ such that

$$
\begin{gathered}
\inf f \leq f^{(K)} \leq \sup f, \quad\left\|\partial_{h_{i}} f^{(K)}\right\|_{L^{\infty}} \leq 2\left\|\partial_{h_{i}} f\right\|_{L^{\infty}}, \quad \text { and } \\
\lim _{K \rightarrow \infty}\left(\left\|f^{(K)}-f\right\|_{L^{q}(\mu)}+\left\|\partial_{h_{i}} f^{(K)}-\partial_{h_{i}} f\right\|_{L^{q}(\mu)}\right)=0 \quad \text { for all } 1 \leq q<\infty .
\end{gathered}
$$

(ii) (Approximation by Boundedly Supported Functions) Given any $h \in \Omega_{-p}^{R}$ and $f \in C_{0}\left(\Omega_{-p ; k}^{R}\right)$, there exists a sequence $\left\{f^{(K)}\right\}_{K \in \mathbb{N}} \subset C_{0}^{1}\left(\Omega_{-p ; k}^{R} ; h\right)$ such that

$$
\begin{array}{ll}
\inf f \leq f^{(K)} \leq \sup f, \quad f^{(K)} \underset{K \rightarrow \infty}{\longrightarrow} f & \text { pointwise on } \Omega_{-p}^{R} \\
\text { and thus } \lim _{K \rightarrow \infty}\left\|f^{(K)}-f\right\|_{L^{q}(\mu)}=0 & \text { for all } 1 \leq q<\infty .
\end{array}
$$

If, additionally, $f$ has a bounded right derivative $\partial_{h}^{+} f: \Omega_{-p}^{R} \rightarrow \mathbb{R}$, then also

$$
\begin{array}{lll}
\left|\partial_{h} f^{(K)}\right| \leq \sup \left|\partial_{h}^{+} f\right|, \quad \partial_{h} f^{(K)} \underset{K \rightarrow \infty}{\longrightarrow} \partial_{h}^{+} f & \text { pointwise on } \Omega_{-p}^{R} \\
\text { and thus } \lim _{K \rightarrow \infty}\left\|\partial_{h} f^{(K)}-\partial_{h}^{+} f\right\|_{L^{q}(\mu)}=0 & \text { for all } 1 \leq q<\infty .
\end{array}
$$

(iii) ( $L^{q}$-Approximation) Given any $h \in \Omega_{-p}^{R}$, each of the sets $\mathcal{F} C_{b}^{\infty}\left(\Omega ; \mathbb{Z}^{d} \times S_{\beta}\right)$, $\mathcal{F} C_{b}^{\infty}(\Omega ; \mathcal{H})$ and $C_{0}^{1}\left(\Omega_{-p ; k}^{R} ; h\right)$ is dense in all spaces $L^{q}(\mu), 1 \leq q<\infty$. Moreover, for $0 \leq f \in L^{\infty}(\mu)$ the corresponding sequences $\left\{f^{(K)}\right\}_{K \in \mathbb{N}}, \lim _{K \rightarrow \infty}\left\|f^{(K)}-f\right\|_{L^{q}(\mu)}=0$, can be chosen so that

$$
0 \leq \inf f \leq f^{(K)} \leq \sup f \quad \text { and } \quad \lim _{K \rightarrow \infty} f^{(K)}=f \quad(\mu \text {-a.e. }) .
$$

Proof. (i) First, we set

$$
\left.g^{(K)}:=f\left(\mathbb{P}_{\Lambda_{K}}\left(\mathbb{M}_{K}\right)\right) \in \mathcal{F} C_{b}\left(\Omega ; \mathbb{Z}^{d+1}\right)\right) \cap C_{b}^{1}\left(\Omega_{-p}^{R} ; h_{i}\right),
$$

where

$$
\left(\mathbb{M}_{K}(\omega)\right)_{k}:=\mathbb{M}_{K}\left(\omega_{k}\right), \quad\left(\mathbb{P}_{\Lambda_{K}} \omega\right)_{k}:=\left\{\begin{array}{rr}
\omega_{k}, & k \in \Lambda_{K} \\
0, & \text { otherwise }
\end{array}\right.
$$


and $\Lambda_{K}, K \in \mathbb{N}$, are bounded domains exhausting $\mathbb{Z}^{d}$. Based on the properties (3.26)(3.28) of the Cesàro partial sums $\mathbb{M}_{K}\left(\omega_{k}\right)$ in the space of continuous loops $C_{\beta}$, it is easy to check that $\left\{g^{(K)}\right\}_{K \in \mathbb{N}}$ satisfies

$$
\begin{gathered}
\inf f \leq g^{(K)} \leq \sup f, \quad\left\|\partial_{h_{i}} g^{(K)}\right\|_{L^{\infty}} \leq\left\|\partial_{h_{i}} f\right\|_{L^{\infty}}, \quad \text { and } \\
g^{(K)} \rightarrow f, \partial_{h_{i}} g^{(K)} \rightarrow \partial_{h_{i}} f \text { pointwise on } \Omega_{-p}^{R} \text { when } K \rightarrow \infty .
\end{gathered}
$$

Next, noting that any cylinder function $g \in \mathcal{F} C(\Omega ; \mathcal{H})$ is of the form $(4.29)$ with some $g_{L} \in C\left(\mathbb{R}^{L}\right)$ and using a standard mollifier argument on $\mathbb{R}^{L}$, for each $g^{(K)}$ defined above one can construct an approximating sequence (in the sense of (4.36)) $\left\{f^{(N)}\right\}_{N \in \mathbb{N}}:=$ $\left\{g^{(K, N)}\right\}_{N \in \mathbb{N}} \subset \mathcal{F} C_{b}^{\infty}(\Omega ; \mathcal{H})$. Finally, we take into account that for any $\omega \in \Omega$ the Riemann integral $\left\langle\omega, h_{i}\right\rangle_{\mathcal{H}}:=\int_{S_{\beta}} \omega_{k}(\tau) \varphi_{n}(\tau) d \tau$ can be approximated by its partial sums in the following way:

$$
\begin{gathered}
\left\langle\omega, h_{i}\right\rangle_{\mathcal{H}}=\lim _{M \rightarrow \infty} \frac{\beta}{M} \sum_{m=0}^{M-1} \varphi_{n}\left(\frac{m}{M} \beta\right) \cdot\left\langle\omega, \delta_{\left(k, \frac{m}{M} \beta\right)}\right\rangle_{\mathcal{H}}, \\
\partial_{h_{i}}\left\langle\omega, h_{i}\right\rangle_{\mathcal{H}}=\lim _{M \rightarrow \infty} \frac{\beta}{M} \sum_{m=0}^{M-1} \varphi_{n}^{2}\left(\frac{m}{M} \beta\right)=\left|\varphi_{n}\right|_{H}^{2}=1, \\
\frac{\beta}{M} \sum_{m=0}^{M-1} \varphi_{n}^{2}\left(\frac{m}{M} \beta\right) \leq \beta \cdot\left|\varphi_{n}\right|_{L_{\beta}^{\infty}}^{2} \leq 2, \quad \forall M, n \in \mathbb{N} .
\end{gathered}
$$

By Lebesgue's dominated convergence theorem, this latter also enables us to approximate each $g^{(K, N)} \in \mathcal{F} C_{b}^{\infty}(\Omega ; \mathcal{H})$ by $\left\{g^{(K, N, M)}\right\}_{M \in \mathbb{N}} \subset \mathcal{F} C_{b}^{\infty}\left(\Omega ; \mathbb{Z}^{d} \times S_{\beta}\right)$ in the sense of (4.32).

(ii) Here one can put, for instance,

$$
\begin{gathered}
f^{(K)}(\omega):=K \int_{0}^{1 / K} f(\omega+\theta h) d \theta \\
\text { with } \partial_{h} f^{(K)}(\omega)=K\left[f\left(\omega+\frac{1}{K} h\right)-f(\omega)\right] .
\end{gathered}
$$

Then it is straightforward to show that the functions $\left\{f^{(K)}\right\}_{K \in \mathbb{N}} \subset C_{0}^{1}\left(\Omega_{-p ; k}^{R} ; h\right)$ satisfy the required assumptions (4.33) and (4.34).

(iii) In this respect we recall the regularity property of Borel measures $\mu \in \mathcal{M}(X)$ on a Polish space $X$, according to which $\mu(\Delta)=\sup \{\mu(B) \mid B \subseteq \Delta, B$ is closed $\}, \forall \Delta \in \mathcal{B}(X)$ (cf. [RS75a, Subsect. IV.4]). In our situation this standardly implies that the set $C_{b}\left(\Omega_{-p ; k}^{R}\right)$ (and thus, by an obvious cut-off argument, also its subset $C_{0}\left(\Omega_{-p ; k}^{R}\right)$ ) is dense in all $L^{q}(\mu), 1 \leq q<\infty$, as well as in $L^{\infty}(\mu)$ (the latter is only in the sense of (4.35)). Starting from this point, in order to construct the desired approximation of $f \in C_{0}\left(\Omega_{-p ; k}^{R}\right)$ by functions from $\mathcal{F} C_{b}^{\infty}(\Omega ; \mathcal{H}), \mathcal{F} C_{b}^{\infty}\left(\Omega ; \mathbb{Z}^{d} \times S_{\beta}\right)$ or resp. $C_{0}^{1}\left(\Omega_{-p ; k}^{R} ; h\right)$, one can, e.g., repeat the previous arguments from the proof of Assertions (i) resp. (ii).

4.3. Partial logarithmic derivatives. Given a probability measure $d \mu(x)=$ $\exp \{-\rho(x)\} d x$ on $\mathbb{R}^{n}$ with (smooth enough) density $\rho$, the vector field $b=\left(b_{l}\right)_{l=1}^{n}:=$ $-\nabla \rho: \mathbb{R}^{n} \rightarrow \mathbb{R}^{n}$ is usually called a logarithmic gradient of $\mu$. Equivalently, one can define its components $b_{l}$, the so-called partial logarithmic derivatives along the basis vectors $e_{l}$, through

$$
b_{l}(x):=\left(\ln \frac{d \mu\left(x+\theta e_{l}\right)}{d \mu(x)}\right)_{\theta=0}^{\prime}, \quad x=\left(x_{l}\right)_{l=1}^{n}=\sum_{l=1}^{n} x_{l} e_{l} \in \mathbb{R}^{n} .
$$


Whereas for Gibbs measures $\mu \in \mathcal{G}_{t}$ the presentation (3.21) with the Euclidean "density" $\mathcal{I}(\omega)$ has a heuristic sense only, below we will use their rigorous description via RadonNikodým derivatives (4.7) in order to modify definition (4.37) to the infinite-dimensional case.

Namely, with regard to Lemma 4.1, we define the partial logarithmic derivative of (all) measures $\mu \in \mathcal{G}_{t}$ along the fixed direction $h_{i}=e_{k} \otimes \varphi_{n}, i=(k, n) \in \mathbb{Z}^{d+1}$, as a mapping $b_{i}: \Omega_{t} \rightarrow \mathbb{R}$

$$
b_{i}(\omega):=\partial_{h_{i}} a_{h_{i}}(\omega)=\left(a_{\theta h_{i}}(\omega)\right)_{\theta=0}^{\prime}=-\left(A_{\beta} \varphi_{n}, \omega_{k}\right)_{H}-\left(F_{k}^{V, W}(\omega), \varphi_{n}\right)_{H} .
$$

Here $F_{k}^{V, W}: \Omega_{t} \rightarrow L_{\beta}^{R^{\prime}}$ (with $R^{-1}+\left(R^{\prime}\right)^{-1}=1$ ) is a nonlinear Nemytskii-type operator acting by

$$
\begin{aligned}
F_{k}^{V, W}(\omega) & :=F_{k}^{V_{k}}\left(\omega_{k}\right)+F_{k}^{W}(\omega) \\
& :=V_{k}^{\prime}\left(\omega_{k}\right)+\left.\sum_{M=2}^{N} \sum_{\substack{\left\{k_{1}, \ldots, k_{M}\right\} \subset \mathbb{Z}^{d} \\
k_{1}:=k}} \partial_{1} W_{\left\{k_{1}, \ldots, k_{M}\right\}}\left(q_{k_{1}}, \ldots, q_{k_{M}}\right)\right|_{\substack{q_{l}=\omega_{l} \\
1 \leq l \leq M}} .
\end{aligned}
$$

Lemma 4.5. For all $i=(k, n), i^{\prime}=\left(k^{\prime}, n^{\prime}\right) \in \mathbb{Z}^{d+1}$ and $p>d$,

$$
F_{k}^{V} \in C_{b, l o c}^{1}\left(C_{\beta} \rightarrow C_{\beta} ; \varphi_{n^{\prime}}\right), \quad F_{k}^{W} \in C_{b, l o c}^{1}\left(\mathcal{L}_{-p}^{R} \rightarrow L_{\beta}^{R^{\prime}} ; h_{i^{\prime}}\right),
$$

and thus the partial logarithmic derivatives (4.38) give rise to smooth mappings

$$
b_{i} \in C_{b, l o c}^{1}\left(\Omega_{-p ; k}^{R} ; h_{i^{\prime}}\right) .
$$

Proof. For $V \in C_{b, l o c}^{2}(\mathbb{R})$ the properties of the operators $F_{k}^{V}$ in the space $C_{\beta}$ are well known: namely, they are (Fréchet) differentiable and

$$
\partial_{\varphi} F_{k}^{V}(v):=V_{k}^{\prime \prime}(v) \cdot \varphi, \quad \forall \varphi \in C_{\beta} .
$$

For fixed $k, k^{\prime} \in \mathbb{Z}^{d}$, along with $F_{k}^{W}$ let us introduce one more Nemytskii-type operator $\partial_{k^{\prime}} F_{k}^{V, W}: \Omega_{t} \rightarrow L_{\beta}^{R^{\prime}}$ by

$$
\begin{aligned}
& \partial_{k^{\prime}} F_{k}^{V, W}(\omega):=\partial_{k^{\prime}} F_{k}^{V}\left(\omega_{k}\right)+\partial_{k^{\prime}} F_{k}^{W}(\omega) \\
& V_{k}^{\prime \prime}\left(\omega_{k}\right)+\left.\sum_{M=2}^{N} \sum_{\substack{\left\{k_{1}, \ldots, k_{M}\right\} \subset \mathbb{Z}^{d} \\
k_{1}:=k}} \partial_{1}^{2} W_{\left\{k_{1}, \ldots, k_{M}\right\}}\left(\omega_{k_{1}}, \ldots, \omega_{k_{M}}\right)\right|_{\substack{q_{l}=\omega_{l} \\
1 \leq l \leq M}}, \quad k=k^{\prime}, \\
& \left.\sum_{M=2}^{N} \sum_{\substack{\left\{k_{1}, \ldots, k_{M}\right\} \subset \mathbb{Z}^{d} \\
k_{1}:=k, k_{2}:=k^{\prime}}} \partial_{1,2}^{2} W_{\left\{k_{1}, \ldots, k_{M}\right\}}\left(\omega_{k_{1}}, \ldots, \omega_{k_{M}}\right)\right|_{\substack{q_{l}=\omega_{l} \\
1 \leq l \leq M}}, k \neq k^{\prime} .
\end{aligned}
$$

Then by $\left(\mathbf{W}_{\mathbf{i i}, \mathbf{i i i}}\right),(\mathbf{J})$ and Lemma 3.4 applied to the RHS in (4.39) and (4.42) we get that

$$
\begin{aligned}
\max & \left\{\left|F_{k}^{W}(\omega)\right|_{L_{\beta}^{R^{\prime}}},\left|\partial_{k^{\prime}} F_{k}^{W}(\omega)\right|_{L_{\beta}^{R^{\prime}}}\right\} \\
& \leq 2^{R-1}\left[\sum_{j \neq k} \tilde{J}_{k, j}\left|\omega_{j}\right|_{L_{\beta}^{R}}^{R-1}+\|J\|_{0}\left(\left|\omega_{j}\right|_{L_{\beta}^{R}}^{R-1}+|\mathbf{1}|_{L_{\beta}^{R^{\prime}}}\right)\right] \\
& \leq 2^{R-1}\left[\|\mathbf{J}\|_{p(R-1)}(1+|k|)^{p(R-1)}\|\omega\|_{-p, R}^{R-1}+\|\mathbf{J}\|_{0}|\mathbf{1}|_{L_{\beta}^{R^{\prime}}}\right]
\end{aligned}
$$


with the matrix $\left\{\tilde{J}_{k, j}\right\}_{k, j \in \mathbb{Z}^{d}}$ defined by (3.45). Hence, obviously,

$$
\begin{aligned}
\max & \left\{\left|\left(F_{k}^{W}(\omega), \varphi_{n}\right)_{H}\right|,\left|\left(\partial_{h_{i}} F_{k}^{W}(\omega), \varphi_{n}\right)_{H}\right|\right\} \\
& \leq 2^{R-1} \kappa_{R}\left[\sum_{j \in \mathbb{Z}^{d}} \tilde{J}_{k, j}\left|\omega_{j}\right|_{L_{\beta}^{R}}^{R}+\|\mathbf{J}\|_{0}\left(\left|\omega_{k}\right|_{L_{\beta}^{R}}^{R}+\beta+1\right)\right] .
\end{aligned}
$$

Since the series in the RHS of (4.43) converges uniformly on balls in $\mathcal{L}_{-p}^{R}$, this standardly yields (cf., e.g., Ca67, Theorem 3.6.2]) that

$$
F_{k}^{W} \in C_{b, l o c}^{1}\left(\mathcal{L}_{-p}^{R} \rightarrow L_{\beta}^{R^{\prime}} ; h_{i^{\prime}}\right) \quad \text { with } \quad \partial_{h_{i^{\prime}}} F_{k}^{W}(\omega)=\partial_{k^{\prime}} F_{k}^{W}(\omega) \cdot \varphi_{n^{\prime}} .
$$

Thus, from the previous discussion it is evident that $b_{i^{\prime}} \in C_{b, l o c}^{1}\left(\Omega_{-p ; k}^{R} ; h_{i^{\prime}}\right)$ with

$$
\partial_{h_{i^{\prime}}} b_{i}=-\delta_{k-k^{\prime}}\left(\lambda_{n}+V_{k}^{\prime \prime}\left(\omega_{k}\right), \varphi_{n} \varphi_{n^{\prime}}\right)_{H}-\left(\partial_{k^{\prime}} F_{k}^{W}(\omega), \varphi_{n} \varphi_{n^{\prime}}\right)_{H}
$$

In particular, taking into account (3.25) and (4.43), one has merely the following bounds on the growth of the logarithmic derivatives:

$$
\left|b_{i}(\omega)\right| \leq \kappa_{\infty}\left\{\lambda_{n}\left|\omega_{k}\right|_{L_{\beta}^{1}}+\left|F_{k}^{V, W}(\omega)\right|_{L_{\beta}^{1}}\right\} \leq C_{i}\left\{1+\left|\omega_{k}\right|_{L_{\beta}^{1}}+\left|V_{k}^{\prime}\left(\omega_{k}\right)\right|_{L_{\beta}^{1}}+\|\omega\|_{-p, R}^{R-1}\right\},
$$

$$
\left|\partial_{h_{i^{\prime}}} b_{i}(\omega)\right| \leq \lambda_{n}+\kappa_{\infty}^{2}\left|\partial_{k^{\prime}} F_{k}^{V, W}(\omega)\right|_{L_{\beta}^{1}} \leq C_{i}^{\prime}\left\{1+\left|V_{k}^{\prime \prime}\left(\omega_{k}\right)\right|_{L_{\beta}^{1}}+\|\omega\|_{-p, R}^{R-1}\right\}
$$

with some absolute constants $C_{i}, C_{i}^{\prime} \in(0, \infty)$ depending on $i \in \mathbb{Z}^{d+1}$ and also on chosen $p>d$.

Combining Lemmas 4.1 and 4.5, by a straightforward calculation one gets the following useful relations between the Radon-Nikodým and logarithmic derivatives.

Corollary 4.6. Under the assumptions on the interaction potentials imposed in Definition 3.1,

$$
\partial_{h_{i}} a_{\theta h_{i}}(\omega)=a_{\theta h_{i}}(\omega)\left[b_{i}\left(\omega+\theta h_{i}\right)-b_{i}(\omega)\right], \quad \frac{\partial}{\partial \theta} a_{\theta h_{i}}(\omega)=a_{\theta h_{i}}(\omega) b_{i}\left(\omega+\theta h_{i}\right),
$$

and thus $a_{\theta h_{i}}$ can be recovered from $b_{i}$ through

$$
a_{\theta h_{i}}(\omega)=\exp \int_{0}^{\theta} b_{i}\left(\omega+\vartheta h_{i}\right) d \vartheta
$$

Remark 4.7. (i) In relation to the further applications in Subsect. 7 below, it is important to note that the pointwise coercivity and growth assumptions on the one-particle potentials $V_{k}$ imply the corresponding properties for the operators $F_{k}^{V}$ w.r.t. the tangent space $H:=L_{\beta}^{2}$. Namely, from $\left(\mathbf{V}_{\mathbf{i}-\mathbf{v}}\right)$ we have uniformly for all $i=(k, n) \in \mathbb{Z}^{d+1}$ and $\omega \in \Omega_{-p}^{R}$ :

$$
\left.\left(F_{k}^{V}\left(\omega_{k}\right), \omega_{k}\right)_{H} \begin{array}{r}
K_{1}^{-1}\left[\kappa_{\infty}^{-1}\left|\left(F_{k}^{V}\left(\omega_{k}\right), \varphi_{n}\right)_{H}\right|+\kappa_{\infty}^{-2}\left|\left(\partial_{n} F_{k}^{V}\left(\omega_{k}\right), \varphi_{n}\right)_{H}\right|-L_{1} \beta\right], \\
K_{2}^{-1}\left[\kappa_{\infty}^{-1}\left|\left(\partial_{n} F_{k}^{V}\left(\omega_{k}\right), \omega_{k}\right)_{H}\right|-L_{2} \beta\right], \\
K_{1}^{-1}\left[\mid F_{k}^{V}\left(\left.\omega_{k}\right|_{L_{\beta}^{1}}-L_{1} \beta\right],\right. \\
K_{3}^{-1}\left[\left|\omega_{k}\right|_{L_{\beta}^{R}}^{R}-L_{3} \beta\right], \\
K_{4}^{-1}\left[\left|\omega_{k}\right|_{H}^{2}-L_{4} \beta\right]
\end{array}\right\}
$$

and

$$
\mid\left(\left.\partial_{\varphi_{n}} F_{k}^{V}\left(\omega_{k}\right)\right|_{L_{\beta}^{R^{\prime}}} \leq \kappa_{\infty}\left[K_{0}\left(\left|F_{k}^{V}\left(\omega_{k}\right)\right|_{L_{\beta}^{R^{\prime}}}+\left|\omega_{k}\right|_{L_{\beta}^{R}}^{R-1}\right)+L_{0}|\mathbf{1}|_{L_{\beta}^{R^{\prime}}}\right]\right.
$$


On the other hand, together with Young's inequality, (4.43) implies the following upper bound for the mappings $F_{k}^{W}$ :

$$
\begin{array}{r}
\max \left\{\left|F_{k}^{W}(\omega)\right|_{L_{\beta}^{R^{\prime}}},\left|\left(F_{k}^{W}(\omega), \omega_{k}\right)_{H}\right|, \kappa_{\infty}^{-1} \cdot\left|\partial_{h_{i}} F_{k}^{W}(\omega)\right|_{L_{\beta}^{R^{\prime}}}, \kappa_{\infty}^{-1} \cdot\left|\left(\partial_{h_{i}} F_{k}^{W}(\omega), \omega_{k}\right)_{H}\right|\right\} \\
\leq 2^{R}\left[\sum_{j \in \mathbb{Z}^{d}} \tilde{J}_{k, j}\left|\omega_{j}\right|_{L_{\beta}^{R}}^{R}+\|\mathbf{J}\|_{0}\left(\left|\omega_{k}\right|_{L_{\beta}^{R}}^{R}+\beta+1\right)\right] .
\end{array}
$$

These estimates will be essential, e.g., for the proof of Theorems 7.5 and 7.6 below.

(ii) Of course, one can also define the "generalized" logarithmic gradient of the measures $\mu \in \mathcal{G}_{t}$ as a measurable vector field $b:=\left(b_{k}\right)_{k \in \mathbb{Z}^{d}}$ with components

$$
\Omega_{-p}^{R} \ni \omega \rightarrow b_{k}(\omega):=\sum_{n \in \mathbb{Z}} b_{(k, n)}(\omega) \cdot \varphi_{n}=-A_{\beta} \omega_{k}-F_{k}^{V, W}(\omega) \in W_{\beta}^{2,-2} .
$$

But even though the (important for the sequel) coercivity estimate (4.47) for its nonlinear components $F_{k}^{V, W}$ holds, it cannot help us to control the coercivity properties of the logarithmic gradient $b$ as a whole w.r.t. the given tangent space $\mathcal{H}:=l^{2}\left(\mathbb{Z}^{d}\right) \otimes L_{\beta}^{2}$ (in contrast with the technique suggested for classical Gibbs measures $\mu_{c l}$ on $\mathcal{S}^{\prime}\left(\mathbb{Z}^{d}\right)$ in AKRT99, AKRT00). The reason is that, taken for every $k \in \mathbb{Z}^{d}$, the bilinear form $\left(A_{\beta} \omega_{k}, \omega_{k}\right)_{H}$ is defined only on a $\mu$-measure zero set of $\omega_{k} \in W_{\beta}^{2,1}$. Thus in the next Sects. 5 and 6 we separately have to do a "lattice analysis", analogous to the one for classical Gibbs states and depending on the coercivity properties in $\mathcal{H}$ of the Nemytskii operators $F_{k}^{V, W}$, as well as an additional "single spin space analysis", taking into account the spectral properties in $L_{\beta}^{2}$ of the elliptic operator $A_{\beta}$ in the linear part of the logarithmic derivatives (4.38).

4.4. Integration by parts (IbP) description of Euclidean Gibbs measures. Next we shall show that, if the interaction potentials are smooth (as they are in our case), the flow characterization (3.9) of $\mu \in \mathcal{G}_{t}$ is equivalent to their definition as differentiable measures satisfying (in the distributional sense by pairing both sides with proper test functions $f$ ) the integration by parts (for short, IbP) formulas

$$
\partial_{h_{i}} \mu(d \omega)=b_{i}(\omega) \mu(d \omega), \quad i \in \mathbb{Z}^{d+1},
$$

with the (given by (4.38)) logarithmic derivatives $b_{i}$ along basis vectors $h_{i} \in \operatorname{bas}(\mathcal{H})$.

So, we return to formula (4.23). The principal problem (remaining open until Theorem 7.6 below) is here that we do not know a priori whether $b_{i} \in L^{1}(\mu)$. This information is needed before a standard theory of differentiable measures (see, e.g., [Bo97]) can be applied. Thus, based on the previous discussion in Subsect. 4.2, we first have to find a large enough number of functions $f$ (which, in fact, will be $\mu$-a.e. differentiable according to Remark 4.10 (ii)), such that certainly $f b_{i} \in L^{\infty}(\mu)$ and passage to the limit in the both sides of (4.23) is correct.

Definition 4.8. Fixing basis vectors $h_{i}, i=(k, n) \in \mathbb{Z}^{d+1}$, we define (conventionally in this paper) a set of test functions

$$
C_{b}^{1, \pm}\left(\Omega_{-p}^{R} ; h_{i}\right):=\left\{\begin{array}{l|c}
f(\omega)=g(\omega) \chi_{2}\left(\left|\omega_{k}\right| C_{\beta},\|\omega\|_{-p, R}\right) \\
f: \Omega_{-p}^{R} \rightarrow \mathbb{R} \mid \begin{array}{c}
\operatorname{with} \forall g \in C^{1}\left(\Omega_{-p}^{R} ; h_{i}\right), \forall \chi_{2} \in C^{1}\left(\mathbb{R}^{2}\right) \\
\sup _{\Omega_{-p}^{R}}\left(|f|+\left|\partial_{h_{i}}^{+} f\right|+\left|\partial_{h_{i}}^{-} f\right|\right)<\infty
\end{array}
\end{array}\right\}
$$


Its subsets $C_{d e c}^{1, \pm}\left(\Omega_{-p}^{R} ; h_{i}\right) \supset C_{0}^{1, \pm}\left(\Omega_{-p}^{R} ; h_{i}\right)$ are defined below. Namely, let $C_{d e c}^{1, \pm}\left(\Omega_{-p}^{R} ; h_{i}\right)$ consist of those $f \in C_{b}^{1, \pm}\left(\Omega_{-p}^{R} ; h_{i}\right)$ which satisfy the extra decay condition

$$
\sup _{\omega \in \Omega_{-p}^{R}}\left|f(\omega)\left(1+\left|\omega_{k}\right|_{L_{\beta}^{R^{\prime}}}+\left|F_{k}(\omega)\right|_{L_{\beta}^{R^{\prime}}}\right)\right|<\infty .
$$

Respectively, $C_{0}^{1, \pm}\left(\Omega_{-p}^{R} ; h_{i}\right)$ consists of those (boundedly supported) functions $f \in$ $C_{b}^{1, \pm}\left(\Omega_{-p}^{R} ; h_{i}\right)$ which can be written in the form

$$
f(\omega)=g(\omega) \cdot \chi_{1}\left(\|\omega\|_{-p, R ; k}\right) \quad \forall g \in C_{b}^{1}\left(\Omega_{-p}^{R} ; h_{i}\right), \forall \chi_{1} \in C_{0}^{1}(\mathbb{R}) .
$$

Note that, by the chain rule and relation (4.26), for any $f \in C_{0}^{1, \pm}\left(\Omega_{-p}^{R} ; h_{i}\right)$ the derivatives $\partial_{h_{i}}^{+} f$ on the right and $\partial_{h_{i}}^{-} f$ on the left exist as globally bounded functions on $\Omega_{-p}^{R}$ (but not necessarily continuous, i.e., possibly $\partial_{h_{i}}^{+} f \notin C\left(\Omega_{-p}^{R}\right)$ ). If $\mu\left(\Omega_{-p}^{R}\right)=1$, then obviously every set $C_{0}^{1, \pm}\left(\Omega_{-p}^{R} ; h_{i}\right)$ is dense in all $L^{q}(\mu), q \in[1, \infty)$ (and also in $L^{\infty}(\mu)$ w.r.t. the $\mu$-a.e. convergence (4.35)) due to the corresponding density property of the sets $C_{0}^{1}\left(\Omega_{-p ; k}^{R} ; h_{i}\right) \subset C_{b}^{1}\left(\Omega_{-p}^{R}\right)$ (cf. Lemma $4.4(\mathrm{iii})$ ).

Having introduced such special classes of test functions, now we are able to prove some (in fact, preliminary) version of the (IbP)-characterization for Euclidean Gibbs measures given by Proposition 4.9. It is inspired by the well-known fact (cf., e.g., [Sk84, Be85, DaS88) that every probability measure $\mu$ on a vector space $X$, which is differentiable along some direction $h \in X$ with corresponding logarithmic derivative $b_{h} \in L^{1}(\mu)$, is for sure also quasi-invariant w.r.t. all shifts $x \mapsto x+\theta h$. However, the new difficulty and the principal difference compared with the above-mentioned papers is that no assumptions on the global integrability of the logarithmic derivatives $b_{h_{i}}$ are imposed here. Moreover, the test functions $f$, for this reason chosen to have bounded support, are not better than partially differentiable on the left and right. Instead, we shall crucially use the proper approximation procedure (cf. Lemma 4.4) and the observation from Lemma 4.1 that $a_{\theta h_{i}}, b_{h_{i}}$ are continuous locally bounded functions on $\Omega_{-p}^{R}$.

Proposition 4.9 ((IbP)-Characterization of Tempered Gibbs Measures). Denote by $\mathcal{M}_{t}^{b}$ the set of all probability measures $\mu$ on $(\Omega, \mathcal{B}(\Omega))$ which satisfy the temperedness condition (3.64) with some fixed $p=p(\mu)>d$ and for any $i \in \mathbb{Z}^{d+1}$ the (IbP)-formula

$$
\int_{\Omega} \partial_{h_{i}}^{ \pm} f(\omega) d \mu(\omega)=-\int_{\Omega} f(\omega) b_{i}(\omega) d \mu(\omega)
$$

for all functions $f \in C_{0}^{1, \pm}\left(\Omega_{-p}^{R} ; h_{i}\right)$. Then

$$
\mathcal{M}_{t}^{b}=\mathcal{M}_{t}^{a}=\mathcal{G}_{t} .
$$

Proof. (i) $\mathcal{M}_{t}^{a} \subseteq \mathcal{M}_{t}^{b}$ : Consider arbitrary $\mu \in \mathcal{M}^{a}$ supported on some $\Omega_{-p}^{R}$ with $p>d$. Then (4.22) holds, in particular, on functions $f \in C_{0}^{1, \pm}\left(\Omega_{-p}^{R} ; h_{i}\right), i=(k, n) \in \mathbb{Z}^{d+1}$, with

$$
\operatorname{supp} f \subset B_{-p ; k}^{R}(\rho)=\left\{\omega \in \Omega_{-p}^{R} \mid\|\omega\|_{-p, R ; k} \leq \rho\right\}, 0<\rho=\rho_{f}<\infty .
$$

According to definition (4.56), for all such $f$,

$$
\sup _{\omega \in \Omega_{-p}^{R}} \sup _{|\theta| \leq 1}\left|\frac{f\left(\omega+\theta h_{i}\right)-f(\omega)}{\theta}\right| \leq \sup _{\omega \in \Omega_{-p}^{R}}\left|\partial_{i}^{ \pm} f\right|<\infty .
$$

On the other hand, from Lemma 4.1,

$$
\sup _{\omega \in B_{-p, k}^{R}(\rho)} \sup _{|\theta| \leq 1}\left|\frac{a_{\theta h_{i}}(\omega)-1}{\theta}\right|<\infty .
$$


Thus, by Lebesgue's dominated convergence theorem one can pass to the limit $\theta \rightarrow \pm 0$ on both sides of (4.22) and get the (IbP)-formula (4.57). This means the desired inclusion $\mu \in \mathcal{M}_{t}^{b}$.

(ii) $\mathcal{M}_{t}^{b} \subseteq \mathcal{M}_{t}^{a}$ : We claim that each $\mu \in \mathcal{M}_{t}^{b}$ is quasi-invariant w.r.t. the shifts $\omega \mapsto \omega+\theta h_{i}, \theta \in \mathbb{R}$, with the Radon-Nikodým derivatives

$$
\frac{d \mu\left(\omega+\theta h_{i}\right)}{d \mu(\omega)}:=\exp \int_{0}^{\theta} b_{i}\left(\omega+\vartheta h_{i}\right) d \vartheta:=a_{\theta h_{i}}(\omega)
$$

(the latter identity in (4.59) is due to Corollary 4.6). So, let $\mu\left(\Omega_{-p}^{R}\right)=1$ and let (4.57) hold for $i=(k, n) \in \mathbb{Z}^{d+1}$. Given any $f \in C_{0}^{1, \pm}\left(\Omega_{-p}^{R} ; h_{i}\right)$, let us define a family of functions indexed by $\theta \in \mathbb{R}$ :

$$
f(\theta, \cdot) \in C_{0}^{1, \pm}\left(\Omega_{-p}^{R} ; h_{i}\right), \quad f(\theta, \omega):=f\left(\omega+\theta h_{i}\right) a_{\theta h_{i}}(\omega) .
$$

Denoting for convenience

$$
I_{f}(\theta):=\int_{\Omega} f(\theta, \omega) d \mu(\omega),
$$

one can check by a direct calculation that $\forall \theta \in \mathbb{R}$,

$$
\exists \frac{d^{ \pm}}{d \theta} I_{f}(\theta)=\int_{\Omega}\left[\partial_{h_{i}}^{ \pm} f\left(\omega+\theta h_{i}\right)+f\left(\omega+\theta h_{i}\right) b_{i}\left(\omega+\theta h_{i}\right)\right] a_{\theta h_{i}}(\omega) d \mu(\omega) .
$$

Substituting the exact expression (4.48) for $\partial_{h_{i}} a_{\theta h_{i}}(\omega)$ in (4.61) and then applying the (IbP)-formula (4.57) to the function $f(\theta, \cdot)$, we find that $\frac{d^{ \pm}}{d \theta} I_{f}(\theta)=0$. In view of the continuity of $\theta \rightarrow I_{f}(\theta)$, the latter yields $I_{f} \equiv$ const, i.e.,

$$
\int_{\Omega} f\left(\omega+\theta h_{i}\right) a_{\theta h_{i}}(\theta, \omega) d \mu(\omega)=\int_{\Omega} f(\omega) d \mu(\omega), \quad \theta \in \mathbb{R} .
$$

Herewith by Fatou's lemma and Lemma 4.4 (iii) we conclude that

$$
E_{\mu}\left(a_{\theta h_{i}}(\theta, \cdot)\right)=\sup \left\{E_{\mu} f\left(\cdot-\theta h_{i}\right) \mid f \in C_{0}^{1, \pm}\left(\Omega_{-p}^{R} ; h_{i}\right), 0 \leq f \leq 1\right\}=1,
$$

as well as that (4.62) holds for all $f \in L^{1}(\mu)$. Since (4.62) and (4.7) are equivalent, the converse inclusion $\mu \in \mathcal{M}_{a}^{t}$ is also proved.

Remark 4.10. The proof of Proposition 4.9 shows the following relations:

(i) The classes of measures $\mu \in \mathcal{M}_{t}$ which satisfy the flow, resp. integration by parts, descriptions coincide, i.e., $\mathcal{M}_{t}^{a}=\mathcal{M}_{t}^{b}$, as soon as $\forall i \in \mathbb{Z}^{d+1}$ there exists $\partial_{\theta} a_{\theta h_{i}} \in$ $C_{b, l o c}\left(\mathbb{R} \times \Omega_{t}\right)$ (and thus also $\left.\partial_{\theta} a_{\theta h_{i}}\right|_{\theta=0}=: b_{i} \in C_{b, l o c}\left(\Omega_{t}\right)$ ). To this end, along with the assumption $V_{k} \in C_{b, l o c}^{1}(\mathbb{R}), k \in \mathbb{Z}^{d}$, one could impose $(\mathbf{J})$ and $\left(\mathbf{W}_{\mathbf{i}, \mathbf{i i}}\right)$ only.

(ii) Let $\mu$ be a probability measure on $\Omega_{-p}^{R}$ such that the (IbP)-formula (4.57) holds for all $f \in C_{0}^{1, \pm}\left(\Omega_{-p}^{R} ; h_{i}\right)$ with fixed $i=(k, n) \in \mathbb{Z}^{d+1}$. Then it also holds for all $f \in C_{0}^{1, \pm}\left(\Omega_{-p^{\prime}}^{R^{\prime}} ; h_{i}\right)$ (even though $\left.C_{0}^{1, \pm}\left(\Omega_{-p^{\prime}}^{R^{\prime}} ; h_{i}\right) \mid \Omega_{-p}^{R} \nsubseteq \nsubseteq C_{0}^{1, \pm}\left(\Omega_{-p}^{R} ; h_{i}\right)\right)$ provided $\partial_{\theta} a_{\theta h_{i}} \in C_{b, l o c}\left(\mathbb{R} \times \Omega_{-p^{\prime} ; k}^{R^{\prime}}\right)$ and $p^{\prime} \geq p>d / 2, R^{\prime} \geq R$.

Corollary 4.11 (Differentiability $\mu$-a.e. of $\left.\left|\omega_{k}\right|_{C_{\beta}}\right)$. Let $\mu\left(\Omega_{-p}^{R}\right)=1$ with some $p>d$, and let the (IbP)-formula (4.56) in the fixed direction $h_{i} \in \operatorname{bas}(\mathcal{H}), i=(k, n) \in \mathbb{Z}^{d+1}$, hold for all functions from $C_{0}^{1, \pm}\left(\Omega_{-p}^{R} ; h_{i}\right)$. Then there exists a Borel set $\Delta \subset \Omega$ such that $\mu(\Delta)=1$, and for all $\omega \in \Delta$,

$$
\partial_{\varphi_{n}}^{+}\left|\omega_{k}\right|_{C_{\beta}}=\partial_{\varphi_{n}}^{-}\left|\omega_{k}\right|_{C_{\beta}} .
$$

Hence, for all $f \in C_{b}^{1, \pm}\left(\Omega_{-p}^{R} ; h_{i}\right)$ and $\omega \in \Delta$, there exists

$$
\partial_{h_{i}} f(\omega)=\partial_{h_{i}}^{ \pm} f(\omega)
$$


and in the formulation of Proposition 4.9 one can simply replace $\partial_{h_{i}}^{ \pm} f$ by $\partial_{h_{i}} f$.

Proof. By the (IbP)-formula (4.57), for all $g \in C_{0}^{1}\left(\Omega_{-p ; k}^{R} ; h_{i}\right)$,

$$
\int_{\Omega} g(\omega) \partial_{h_{i}}^{ \pm}\left|\omega_{k}\right|_{C_{\beta}} d \mu(\omega)=-\int_{\Omega}\left|\omega_{k}\right|_{C_{\beta}}\left[\partial_{h_{i}} g+g b_{i}\right](\omega) d \mu(\omega) .
$$

Since the set $C_{0}^{1}\left(\Omega_{-p ; k}^{R} ; h_{i}\right)$ is dense in $L^{1}(\mu)$ (cf. Lemma $4.4($ iii) $)$, this implies

$$
\mu\left(\left.\omega \in \Omega\left|\partial_{\varphi_{n}}^{+}\right| \omega_{k}\right|_{C_{\beta}}=\partial_{\varphi_{n}}^{-}\left|\omega_{k}\right|_{C_{\beta}}, \quad \forall(k, n) \in \mathbb{Z}^{d+1}\right)=1 .
$$

We note that the identity in (4.65), $\partial_{h_{i}}^{+} f=\partial_{h_{i}}^{-} f$ ( $\mu$-a.e.), might also be derived from a general result in [Ku82, Lemma 1.3] on the so-called stochastic $H$-Gâteaux differentiability of Lipschitz functions $f: B \rightarrow \mathbb{R}$ w.r.t. any positive quasi-invariant measure $\mu$ on an abstract Wiener space $B \supset H \supset B^{*}$.

So, based on Proposition 4.9, instead of Euclidean Gibbs measures $\mu \in \mathcal{G}$ initially defined as random fields on the lattice $\mathbb{Z}^{d}$, we can just study probability measures on $\Omega$ satisfying the (IbP)-formula (4.57) with the (prescribed by (4.38)) logarithmic derivatives $b_{i}, i \in \mathbb{Z}^{d+1}$. Let us stress that these $b_{i}$ only depend on the given potentials $V$ and $W$ and hence are the same for all $\mu \in \mathcal{G}$ associated with the heuristic Hamiltonian (3.1). Solutions $\mu \in \mathcal{M}^{b}$ to the (IbP)-formula (4.57) will also be called symmetrizing measures. For further important connections to reversible diffusion processes and Dirichlet operators in infinite dimensions we refer e.g. to AKR97a, AKR97b, AKRT01, BR01, BRW01 and to Subsect. 5.3 below.

4.5. Further discussion of the (IbP)-formula. Here we present some modifications of the (IbP)-formula (4.57) that will useful in applications. The domain $C_{0}^{1, \pm}\left(\Omega_{-p}^{R}, h_{i}\right)$, on which we have initially proved the (IbP)-characterization of Euclidean Gibbs measures in Proposition 4.9, was chosen for reasons of technical convenience only, and indeed it plays not more than an intermediate role. Among others, the subsequent statement will show that as soon as we have the (IbP)-formula (4.57) in a usual setting with $\partial_{h_{i}} f$ on some "minimal domain" consisting of $h_{i}$-differentiable functions with bounded support (e.g., $\left.C_{0}^{1}\left(\Omega_{-p, k}^{R} ; h_{i}\right)\right)$ or of smooth cylinder functions (e.g., $\mathcal{F} C_{b}^{\infty}\left(\Omega ; \mathbb{Z}^{d} \times S_{\beta}\right)$ or $\left.\mathcal{F} C_{b}^{\infty}(\Omega ; \mathcal{H})\right)$, then we can always extend it, by substituting $\partial_{h_{i}}^{ \pm} f$ for $\partial_{h_{i}} f$, to a "maximal domain" of definition on functions which are differentiable along $h_{i}$ at least on the right or left (e.g., $\left.C_{b}^{1, \pm}\left(\Omega_{-p}^{R}, h_{i}\right)\right)$. We recall that the spaces of test functions used here have already been introduced respectively in Subsections 4.2.1, 4.2.3 and by Definition 4.8.

Proposition 4.12 (Equivalent Domains for (IbP)-Formula). Let $\mu\left(\Omega_{-p}^{R}\right)=1$ with some $p>d$, and let us fix some direction $h_{i} \in \operatorname{bas}(\mathcal{H}), i=(k, n) \in \mathbb{Z}^{d+1}$. Then the following assertions hold:

(i) Suppose that the (IbP)-formula (4.57) is valid for functions from $C_{0}^{1, \pm}\left(\Omega_{-p}^{R}, h_{i}\right)$. Then it extends by continuity to $C_{d e c}^{1, \pm}\left(\Omega_{-p}^{R}, h_{i}\right)$ and, in particular, to $C_{0}^{1}\left(\Omega_{-p, k}^{R} ; h_{i}\right) \subset$ $C_{\text {dec }}^{1, \pm}\left(\Omega_{-p}^{R}, h_{i}\right)$. If, moreover, we know that $b_{i} \in L^{1}(\mu)$, then (4.57) further extends from $C_{0}^{1, \pm}\left(\Omega_{-p}^{R}, h_{i}\right)$ to $C_{b}^{1, \pm}\left(\Omega_{-p}^{R}, h_{i}\right)$, and in particular to all cylinder functions from $\mathcal{F} C_{b}^{\infty}\left(\Omega ; \mathbb{Z}^{d} \times S_{\beta}\right)$ or $\mathcal{F} C_{b}^{\infty}(\Omega ; \mathcal{H})$.

(ii) The inverse statement to (i) is also true. Suppose that the (IbP)-formula (4.57) is valid for functions from $C_{0}^{1}\left(\Omega_{-p, k}^{R} ; h_{i}\right)$. Then it extends by continuity to all $f \in$ $C_{0}^{1, \pm}\left(\Omega_{-p}^{R}, h_{i}\right)$. If $b_{i} \in L^{1}(\mu)$, then (4.57) extends from $\mathcal{F} C_{b}^{\infty}\left(\Omega ; \mathbb{Z}^{d} \times S_{\beta}\right)$, resp. $\mathcal{F} C_{b}^{\infty}(\Omega ; \mathcal{H})$, to $C_{b}^{1, \pm}\left(\Omega_{-p}^{R}, h_{i}\right)$. 
Proof. (i) For any $f \in C_{b}^{1, \pm}\left(\Omega_{-p}^{R}, h_{i}\right)\left(\supset C_{d e c}^{1, \pm}\left(\Omega_{-p}^{R}, h_{i}\right)\right)$, let us take its approximation by $\left\{f^{(K)}\right\}_{K \in \mathbb{N}} \subset C_{0}^{1, \pm}\left(\Omega_{-p}^{R}, h_{i}\right)$ of the form

$$
f^{(K)}(\omega):=f(\omega) \chi_{K}\left(\|\omega\|_{-p, R ; k}\right),
$$

where $\left\{\chi_{K}\right\}_{K \in \mathbb{N}} \subset C_{0}^{1}\left(\mathbb{R}_{+} \rightarrow[0,1]\right)$ is a cut-off sequence with the properties

$$
\begin{gathered}
\chi_{K}(s)=1 \text { for } s \in[0, K], \chi_{K}(s)=0 \text { for } s \in[K+1, \infty) \\
\text { and } \chi_{K+1}(s+1)=\chi_{K}(s) \text { for every } s \geq 0 .
\end{gathered}
$$

The statement follows by Lebesgue's dominated convergence theorem applied, as $K \rightarrow$ $\infty$, to both sides of (4.57) with $f^{(K)}$ replacing $f$.

(ii) By Lemma 4.4 (i) and Lebesgue's convergence theorem, (4.53) extends either from $\mathcal{F} C_{b}^{\infty}\left(\Omega ; \mathbb{Z}^{d} \times S_{\beta}\right)$ or $\mathcal{F} C_{b}^{\infty}(\Omega ; \mathcal{H})$ to the sets $C_{b}^{1}\left(\Omega_{-p}^{R} ; h_{i}\right) \supset C_{b}^{1}\left(\Omega_{-p ; k}^{R} ; h_{i}\right)$ provided $b_{i} \in L^{1}(\mu)$. On the other hand, by Lemma 4.4 (i), (ii) and again by Lebesgue's dominated convergence theorem, (4.57) extends from $C_{0}^{1}\left(\Omega_{-p ; k}^{R} ; h_{i}\right)$ to $C_{0}^{1, \pm}\left(\Omega_{-p}^{R}, h_{i}\right)$. Finally, the passage from $C_{0}^{1, \pm}\left(\Omega_{-p}^{R}, h_{i}\right)$ to $C_{b}^{1, \pm}\left(\Omega_{-p}^{R}, h_{i}\right)$ was just performed in the proof of (i).

Now we present equivalent versions of the (IbP)-formula (4.57) for smooth cylinder functions on $\Omega$. Respectively, it would be important to extend (4.57), when applied to the test functions $f \in \mathcal{F} C_{b}^{1}\left(\Omega ; \mathbb{Z}^{d} \times S_{\beta}\right)$, from the admissible single spin directions $\varphi \in \operatorname{lin}\left\{\varphi_{n}\right\}_{n \in \mathbb{Z}}$ to the Green function $\mathfrak{G}_{\tau}:=A_{\beta}^{-1} \delta_{\tau}, \tau \in S_{\beta}$, of the operator $A_{\beta}$ (for its definition and properties see (3.29)-(3.31)). Heuristically, this is possible for the following reason: even though $\varphi:=\mathfrak{G}_{\tau} \notin \mathcal{D}\left(A_{\beta}\right)$ and hence, in general, $d \mu(\omega+\theta h) \perp d \mu(\omega)$ for $\mu \in$ $\mathcal{M}_{t}^{b}, \theta \in \mathbb{R}$ and $h:=\varphi \otimes e_{k} \in \mathcal{H}$, we have absolute continuity for the corresponding spacetime projections of these measures. Technically, such an extension will be performed through some standard approximation procedure for $\mathfrak{G}_{\tau}$, which also will be needed below in the proof of all our Main Theorems I-III. We also have to assume here that $\mu \in \mathcal{M}_{t}^{b}$ possesses the following a priori integrability properties:

$\left(\mathbf{H}_{\mu}\right)$ For all $i:=(k, n) \in \mathbb{Z}^{d+1}:$

$$
\left|\omega_{k}\right|_{C_{\beta}},\left|F_{k}^{V, W}(\omega)\right|_{L_{\beta}^{1}} \in L^{1}(\mu) \text { and thus also } b_{i} \in L^{1}(\mu) .
$$

As will be shown later in Main Theorem II, Hypothesis $\left(\mathbf{H}_{\mu}\right)$ always hold under Assumptions (W), (J), ( V). Finally we note that local versions of the (IbP)-formula analogous to (4.71) are of common use in the literature on quantum models (see e.g. GJ81]).

Proposition 4.13 ("Local" (IbP)-Formula). Let the measure $\mu \in \mathcal{M}_{t}^{b}$ satisfy Hypothesis $\left(\boldsymbol{H}_{\mu}\right)$. Then the following (IbP)-formulas for cylinder functions from $\mathcal{F} C_{b}^{1}\left(\Omega ; \mathbb{Z}^{d} \times S_{\beta}\right)$, resp. $\mathcal{F} C_{b}^{1}(\Omega ; \mathcal{H})$, hold:

$$
\begin{gathered}
\text { (i) } \sum_{l=1}^{L} \delta_{k-k_{l}} \mathfrak{G}\left(\tau, \tau_{l}\right) \int_{\Omega} \partial_{l} f_{L}\left(\omega_{k}\left(\tau_{1}\right), \ldots, \omega_{k}\left(\tau_{L}\right)\right) d \mu(\omega) \\
=-\int_{\Omega} f_{L}\left(\omega_{k}\left(\tau_{1}\right), \ldots, \omega_{k}\left(\tau_{L}\right)\right)\left(\omega_{k}+A_{\beta}^{-1} F_{k}^{V, W}(\omega)\right)(\tau) d \mu(\omega) ; \\
\text { (ii) } \sum_{l=1}^{L} \delta_{k-k_{l}}\left(A_{\beta}^{-1} \varphi_{n_{l}}\right)(\tau) \int_{\Omega} \partial_{l} f_{L}\left(\left\langle\omega, h_{i_{1}}\right\rangle_{\mathcal{H}}, \ldots,\left\langle\omega, h_{i_{L}}\right\rangle_{\mathcal{H}}\right) d \mu(\omega) \\
=-\int_{\Omega} f_{L}\left(\left\langle\omega, h_{i_{1}}\right\rangle_{\mathcal{H}}, \ldots,\left\langle\omega, h_{i_{L}}\right\rangle_{\mathcal{H}}\right)\left(\omega_{k}+A_{\beta}^{-1} F_{k}^{V, W}(\omega)\right)(\tau) d \mu(\omega)
\end{gathered}
$$

for any $f_{L} \in C_{b}^{1}\left(\mathbb{R}^{L}\right), h \in\left[L_{\beta}^{2}\right]^{d}, n_{l} \in \mathbb{Z}, k, k_{l} \in \mathbb{Z}^{d}, \tau, \tau_{l} \in S_{\beta}, 1 \leq l \leq L$ and $L \in \mathbb{N}$. 
Proof. (i) At first, we shall exploit the assumptions $\left|F_{k}^{V, W}(\omega)\right|_{L_{\beta}^{1}},\left|\omega_{k}\right|_{L_{\beta}^{2}} \in L^{1}(\mu)$ from Hypothesis $\left(\mathbf{H}_{\mu}\right)$. According to Proposition 4.12, (4.57) is then equivalent to the (IbP)formula

$$
\begin{gathered}
\int_{\Omega} \partial_{h_{i}} f(\omega) d \mu(\omega)=\sum_{l=1}^{L} \delta_{k-k_{l}} \varphi_{n}\left(\tau_{l}\right) \int_{\Omega} \partial_{l} f_{L}\left(\omega_{k_{1}}\left(\tau_{1}\right), \ldots, \omega_{k_{L}}\left(\tau_{L}\right)\right) d \mu(\omega) \\
=-\int_{\Omega} f_{L}\left(\omega_{k_{1}}\left(\tau_{1}\right), \ldots, \omega_{k_{L}}\left(\tau_{L}\right)\right)\left[\left(A_{\beta} \varphi_{n}, \omega_{k}\right)_{H}+\left(F_{k}^{V, W}(\omega), \varphi_{n}\right)_{H}\right] d \mu(\omega)
\end{gathered}
$$

valid for all cylinder functions $f \in \mathcal{F} C_{b}^{1}\left(\Omega ; \mathbb{Z}^{d} \times S_{\beta}\right)$ of the form (4.30). Since $T_{\beta}:=$ $\operatorname{lin}\left\{\varphi_{n}\right\}_{n \in \mathbb{Z}}$ is an essential domain in $L_{\beta}^{2}$ for the selfadjoint operator $A_{\beta}$ with $\mathcal{D}\left(A_{\beta}\right)=$ $W_{\beta}^{2,2}$ (see Subsect. 3.3.1 above), (4.73) further extends by continuity to arbitrary $\varphi \in$ $\mathcal{D}\left(A_{\beta}\right)$ as

$$
\begin{aligned}
& \sum_{l=1}^{L} \delta_{k-k_{l}} \varphi\left(\tau_{l}\right) \int_{\Omega} \partial_{l} f_{L}\left(\omega_{k_{1}}\left(\tau_{1}\right), \ldots, \omega_{k_{L}}\left(\tau_{L}\right)\right) d \mu(\omega) \\
& \quad=-\int_{\Omega} f_{L}\left(\omega_{k_{1}}\left(\tau_{1}\right), \ldots, \omega_{k_{L}}\left(\tau_{L}\right)\right)\left[\left(A_{\beta} \varphi, \omega_{k}\right)_{H}+\left(F_{k}^{V, W}(\omega), \varphi\right)_{H}\right] d \mu(\omega) .
\end{aligned}
$$

Now, fixing $\tau \in S_{\beta}$, we would like to replace $\varphi$ in (4.74) by the Green function $\mathfrak{G}_{\tau}:=A_{\beta}^{-1} \delta_{\tau}$. With this purpose we construct the (so-called Yosida) approximation of $\mathfrak{G}_{\tau} \in W_{\beta}^{2,1} \subset C_{\beta}$ by

$$
\varphi_{\tau}^{(K)}:=\left(1+K^{-1} A_{\beta}\right)^{-1} \mathfrak{G}_{\tau} \in \mathcal{D}\left(A_{\beta}\right), \quad K \in \mathbb{N} .
$$

Since $A_{\beta}$ generates a Markovian $C_{0}$-semigroup on $C_{\beta}$, its resolvent has the following properties:

$$
\begin{gathered}
\sup _{K \in \mathbb{N}}\left\|\left(1+K^{-1} A_{\beta}\right)^{-1}\right\|_{C_{\beta} \rightarrow C_{\beta}} \leq 1, \\
\lim _{K \rightarrow \infty}\left|\left(1+K^{-1} A_{\beta}\right)^{-1} v-v\right|_{C_{\beta}}=0, \text { for all } v \in C_{\beta} .
\end{gathered}
$$

Therefore, $\lim _{K \rightarrow \infty}\left|\varphi_{\tau}^{(K)}-\mathfrak{G}_{\tau}\right|_{C_{\beta}}=0$ and, moreover,

$$
\begin{gathered}
\left|\left(A_{\beta} \varphi_{\tau}^{(K)}, \omega_{k}\right)_{H}\right| \leq\left|\omega_{k}\right|_{C_{\beta}}, \\
\lim _{K \rightarrow \infty}\left(A_{\beta} \varphi_{\tau}^{(K)}, \omega_{k}\right)_{H}=\lim _{K \rightarrow \infty}\left(\delta_{\tau},\left(1+K^{-1} A_{\beta}\right)^{-1} \omega_{k}\right)_{H}=\omega_{k}(\tau) .
\end{gathered}
$$

On the other hand, $A_{\beta}^{-1} F_{k}^{V, W}: \Omega_{-p}^{R} \rightarrow C_{\beta}$ and hence

$$
\begin{gathered}
\lim _{K \rightarrow \infty}\left(F_{k}^{V, W}(\omega), \varphi_{\tau}^{(K)}\right)_{H}=\left(A_{\beta}^{-1} F_{k}^{V, W}(\omega)\right)(\tau), \\
\left|\left(F_{k}^{V, W}(\omega), \varphi_{\tau}^{(K)}\right)_{H}\right| \leq\left|F_{k}^{V, W}(\omega)\right|_{L_{\beta}^{1}}\left|\mathfrak{G}_{\tau}\right|_{C_{\beta}} .
\end{gathered}
$$

Finally, taking into account (4.76), (4.77) and the next assumption $\left|\omega_{k}\right|_{C_{\beta}} \in L^{1}(\mu)$ from Hypothesis $\left(\mathbf{H}_{\mu}\right)$, by Lebesgue's dominated convergence theorem, (4.74) extends to (4.71). 
(ii) The proof for the case of cylinder functions $f \in \mathcal{F} C_{b}^{1}\left(\Omega_{\beta} ; \mathcal{H}\right)$ of the form (4.28) is analogous. Namely, under the assumptions $\left|F_{k}^{V, W}(\omega)\right|_{L_{\beta}^{1}},\left|\omega_{k}\right|_{L_{\beta}^{2}} \in L^{1}(\mu)$, the (IbP)formula (4.57) is equivalent to

$$
\begin{aligned}
& \sum_{l=1}^{L}\left\langle\left(\mathbf{1} \otimes A_{\beta}^{-1}\right) h, h_{i_{L}}\right\rangle_{\mathcal{H}} \int_{\Omega} \partial_{l} f_{L}\left(\left\langle\omega, h_{i_{1}}\right\rangle_{\mathcal{H}}, \ldots,\left\langle\omega, h_{i_{L}}\right\rangle_{\mathcal{H}}\right) d \mu(\omega) \\
& \quad=-\int_{\Omega} f_{L}\left(\left\langle\omega, h_{i_{1}}\right\rangle_{\mathcal{H}}, \ldots,\left\langle\omega, h_{i_{L}}\right\rangle_{\mathcal{H}}\right)\left\langle\omega+\left(\mathbf{1} \otimes A_{\beta}^{-1}\right) F^{V, W}(\omega), h\right\rangle_{\mathcal{H}} d \mu(\omega)
\end{aligned}
$$

and, if $\left|\omega_{k}\right|_{C_{\beta}} \in L^{1}(\mu)$, it further extends by continuity to (4.72).

Remark 4.14. For the particular QLS models (2.2) and (2.21) with local interaction, the corresponding (IbP)-characterization (as well as the flow characterization, cf. Remark 4.3 ) is available for all (not necessarily tempered) $\mu \in \mathcal{G}$. So, as was shown in AKPR04, Proposition 2], the (IbP)-formula (4.57) holds (without any a priori information about global integrability of $\left.b_{i}\right)$ for all test functions $f \in C_{b}^{1}\left(\Omega ; h_{i}\right)$ satisfying the extra decay condition (4.55).

\section{Applications of the (IbP)-Formula}

5.1. (IbP)-formula for probability kernels of the local specification. As can immediately be seen from the definitions (3.41)-(3.44) and, for classical lattice systems, has already been mentioned in Ro77, the following flow description for the probability kernels $\pi_{\Lambda}(d \omega \mid \xi)$ of the local specification $\left(\pi_{\Lambda}\right)_{\Lambda \Subset \mathbb{Z}^{d}}$ is true.

Proposition 5.1. Measures $\pi_{\Lambda}(d \omega \mid \xi), \xi \in \Omega_{t}, \Lambda \Subset \mathbb{Z}^{d}$ (as well as the finite-volume Gibbs distributions $\left.\mu_{\Lambda}\left(d \omega_{\Lambda} \mid \xi_{\Lambda^{c}}\right)\right)$ are quasi-invariant w.r.t. shifts

$$
\omega \longmapsto \omega+\theta h_{i}, \text { for all } i=(k, n), k \in \Lambda \Subset \mathbb{Z}^{d}, n \in \mathbb{Z} \text {, and } \theta \in \mathbb{R},
$$

with the same Radon-Nikodým derivatives as those for the corresponding infinite-volume Gibbs measures $\mu \in \mathcal{G}_{t}$.

More precisely, Proposition 5.1 means that for every such admissible shift $\theta h_{i} \in \mathcal{H}$,

$$
\frac{d \pi_{\Lambda}\left(\omega+\theta h_{i} \mid \xi\right)}{d \pi_{\Lambda}(\omega \mid \xi)}=a_{\theta h_{i}}(\omega)>0, \text { for all } \omega \in \Omega_{t}\left(\pi_{\Lambda}(d \omega \mid \xi) \text {-a.e. }\right)
$$

or, equivalently, for all $f \in L^{1}\left(\Omega, \pi_{\Lambda}(d \omega \mid \xi)\right)$,

$$
\int_{\Omega} f(\omega) a_{\theta h_{i}}(\omega) \pi_{\Lambda}(d \omega \mid \xi)=\int_{\Omega} f\left(\omega-\theta h_{i}\right) \pi_{\beta, \Lambda}(d \omega \mid \xi) .
$$

A reasoning similar to that used in the proof of Propositions 4.9 and 4.12 (i) then gives the corresponding infinitesimal version of (5.1):

Proposition 5.2. Measures $\pi_{\Lambda}(d \omega \mid \xi), \xi \in \Omega_{t}, \Lambda \Subset \mathbb{Z}^{d}$ (as well as the finite-volume Gibbs distributions $\mu_{\Lambda}\left(d \omega_{\Lambda} \mid \xi_{\Lambda^{c}}\right)$ on $\left.\Omega_{\Lambda}\right)$ satisfy the $(\mathrm{IbP})$-formula

$$
\int_{\Omega} \partial_{h_{i}}^{ \pm} f(\omega) \pi_{\Lambda}(d \omega \mid \xi)=-\int_{\Omega} f(\omega) b_{i}(\omega) \pi_{\Lambda}(d \omega \mid \xi),
$$

for all $i=(k, n), k \in \Lambda \Subset \mathbb{Z}^{d}, n \in \mathbb{Z}$, and for all functions $f \in \bigcup_{p>d} C_{0}^{1, \pm}\left(\Omega_{-p}^{R} ; h_{i}\right)$.

We note that for the proof of the above Propositions 5.1 and 5.2, as well as of Proposition 5.3 below, it is essential that (as was checked in Lemmas 4.1 and 4.5),

$$
a_{\theta h_{i}}, b_{i} \in C_{b, l o c}\left(\Omega_{-p ; k}^{R}\right), \quad \text { for all } i=(k, n) \in \mathbb{Z}^{d+1}, \theta \in \mathbb{R} .
$$


Hence, if a sequence $\pi_{\Lambda^{(K)}}\left(d \omega \mid \xi^{(K)}\right)$, where $\xi^{(K)} \in \Omega_{-p}^{R}, \Lambda^{(K)} \nearrow \mathbb{Z}^{d}$ as $K \rightarrow \infty$, weakly converges on the Banach space $\Omega_{-p ; k}^{R}$ to some probability measure $\mu_{*}$, one can also pass to the limit on both sides of (5.1) and (5.2). So, for $\mu:=\mu_{*}$ we again have both the flow description (4.7) and the (IbP)-formula (4.57), which hold in any direction $h_{i}$, $i=(k, n) \in \mathbb{Z}^{d+1}$, for all functions $f \in C_{0}^{1}\left(\Omega_{-p ; k}^{R} ; h_{i}\right) \subset C_{0}^{1, \pm}\left(\Omega_{-p}^{R} ; h_{i}\right)$. Combining these properties of $\mu_{*}$ with Propositions 4.2, 4.9 and 4.12, we have thus proved the following:

Proposition 5.3 (Thermodynamic Limit Points are Gibbs). Fix any configuration space $\Omega_{-p}^{R}, p>d$, and consider a sequence of measures $\pi_{\Lambda^{(K)}}\left(d \omega \mid \xi^{(K)}\right), K \in \mathbb{N}$, where $\xi^{(K)} \in$ $\Omega_{-p}^{R}$ and $\Lambda^{(K)} \Subset \mathbb{Z}^{d}, \Lambda^{(K)} \nearrow \mathbb{Z}^{d}$ as $K \rightarrow \infty$. Then each of its accumulation points $\mu_{*}$ w.r.t. the topology of weak convergence of measures on the Polish space $\Omega_{-p}^{R}$, provided such exist, is Gibbs.

In this way, the alternative characterization of Euclidean Gibbs measures enables us to study the existence problem for $\mu \in \mathcal{G}_{t}$ just by showing some uniform estimates on measures $\pi_{\Lambda}(d \omega \mid \xi)$ which certainly imply their tightness.

5.2. Proof of Main Theorems I-III under additional Hypotheses (H) and $\left(\mathbf{H}_{l o c}\right)$. In this subsection we complete the proof of our Main Theorems I-III under the additional Hypotheses $(\mathbf{H})$ and $\left(\mathbf{H}_{l o c}\right)$ concerning the uniform integrability of $\left|\omega_{k}\right|_{L_{\beta}^{2}}$ and $\left|F_{k}(\omega)\right|_{L_{\beta}^{1}}$ w.r.t. tempered Gibbs measures $\mu \in \mathcal{G}_{t}$ and their local specifications $\left\{\pi_{\Lambda}\right\}_{\Lambda \Subset \mathbb{Z}^{d}}$. The verification of these crucial hypotheses (which is one of the hardest parts of this paper) we postpone to Subsect.7.3, after we have available the necessary techniques based on the coercivity properties of the logarithmic derivatives $b_{i}$ and developed in Sections 6 and 7 below. The respective Theorems 7.5 and 7.6 will confirm that both $(\mathbf{H})$ and $\left(\mathbf{H}_{l o c}\right)$ always hold for the system (3.1) as soon as the interaction satisfies Assumptions $(\mathbf{W}),(\mathbf{J})$ and $(\mathbf{V})$ with a proper relation between the parameters involved.

So, within this subsection we assume that

(H) For every $Q \geq 1$,

$$
I_{2 Q}(F):=\sup _{\mu \in \mathcal{G}_{t}} \sup _{k \in \mathbb{Z}^{d}} \int_{\Omega}\left[\left|\omega_{k}\right|_{L_{\beta}^{2}}+\left|F_{k}^{V, W}(\omega)\right|_{L_{\beta}^{1}}\right]^{2 Q} d \mu(\omega)<\infty .
$$

$\left(\mathbf{H}_{l o c}\right)$ For some fixed $Q>1$ and a boundary condition $\xi \in \Omega_{t}$ :

$$
I_{2 Q, \xi}(F):=\sup _{\Lambda \Subset \mathbb{Z}^{d}} \sup _{k \in \Lambda} \int_{\Omega}\left[\left|\omega_{k}\right|_{L_{\beta}^{2}}+\left|F_{k}^{V, W}(\omega)\right|_{L_{\beta}^{1}}\right]^{2 Q} \pi_{\Lambda}(d \omega \mid \xi)<\infty .
$$

Our next aim is to demonstrate that, taking into account the regularity properties of the Green function $\mathfrak{G}\left(\tau, \tau^{\prime}\right)$ of the operator $A_{\beta}$, from the (IbP)-formulas (4.57), resp. (5.2), and Hypotheses $(\mathbf{H})$, resp. $\left(\mathbf{H}_{l o c}\right)$, one can already get uniform estimates on Hölder loop spaces $C_{\beta}^{\alpha}$ for all tempered Gibbs measures $\mu \in \mathcal{G}_{t}$ as well as the existence of the latter. Recall that, according to our previous agreement, $\mathcal{G}_{t}$ denotes either $\mathcal{G}_{(e) t}^{R}$ for the particular QLS models I-III or respectively $\mathcal{G}_{(s) t}^{R}$ for the general model (3.1).

5.2.1. A priori estimates and support properties of Euclidean Gibbs measures on Hölder loops. The following result is the crucial step towards the proof of Main Theorem II:

Lemma 5.4 (Kolmogorov type moment estimates). For given $k \in \mathbb{Z}^{d}$, let a measure $\mu \in \mathcal{M}_{t}$ satisfy the (IbP)-formula (4.57) in all directions $h_{i}$ with $i=(k, n), n \in \mathbb{Z}$, and, moreover, obeys the integrability property

$$
\int_{\Omega}\left[\left|\omega_{k}\right|_{L_{\beta}^{2}}+\left|F_{k}^{V, W}(\omega)\right|_{L_{\beta}^{1}}\right]^{2 Q} d \mu(\omega) \leq I_{2 Q}
$$


with some fixed $Q \in \mathbb{N}$ and $I_{2 Q}:=I_{2 Q}\left(F_{k} ; \mu\right) \in(0, \infty)$. Then (5.3) implies the moment estimates

$$
\begin{gathered}
\int_{\Omega} \omega_{k}^{2 Q}(\tau) d \mu(\omega):=C_{2 Q}<\infty, \\
\int_{\Omega}\left[\omega_{k}(\tau)-\omega_{k}\left(\tau^{\prime}\right)\right]^{2 Q} d \mu(\omega) \leq \Delta C_{2 Q} \cdot \rho^{Q}\left(\tau, \tau^{\prime}\right)
\end{gathered}
$$

for all $\tau, \tau^{\prime} \in S_{\beta}$ and with the absolute (i.e., independent on $\mu$ and $k$ ) constants $C_{2 Q}$, $\Delta C_{2 Q} \in(0, \infty)$. Moreover, $C_{2 Q}$ and $\Delta C_{2 Q}$ themselves are linear functions of $I_{2 Q}$.

Proof. (i) Let us consider the following two families of test functions indexed by $\tau, \tau^{\prime} \in$ $S_{\beta}$ and $\varepsilon>0$ :

$$
\begin{aligned}
f(\omega) & :=f_{\tau, \tau^{\prime}, \varepsilon}(\omega):=\omega_{k}^{2 Q-1}(\tau) Z^{-1}\left(\omega_{k}\right), \\
g(\omega) & :=g_{\tau, \tau^{\prime}, \varepsilon}(\omega):=\left[\Delta \omega_{k}\right]^{2 Q-1} Z^{-1}\left(\omega_{k}\right),
\end{aligned}
$$

where we set for convenience

$$
Z\left(\omega_{k}\right):=Z_{\varepsilon}\left(\omega_{k}\right):=1+\varepsilon\left|\omega_{k}\right|_{C_{\beta}}^{2 Q}, \quad \Delta \omega_{k}:=\Delta \omega_{k}\left(\tau, \tau^{\prime}\right):=\omega_{k}(\tau)-\omega_{k}\left(\tau^{\prime}\right) .
$$

According to Definition 4.7, $f, g \in C_{b}^{1, \pm}\left(\Omega_{-p}^{R} ; h_{i}\right)$ for any $p>d / 2$ and $h_{i}:=e_{k} \otimes \varphi_{n}$, $n \in \mathbb{Z}$. Moreover, using (4.26) and (4.31), one can easily calculate the corresponding derivatives along vectors $h:=e_{k} \otimes \varphi$ with arbitrary $\varphi \in C_{\beta}$ :

$$
\begin{gathered}
\partial_{h}^{ \pm} f(\omega)=(2 Q-1) \omega_{k}^{2 Q-2}(\tau) \varphi(\tau) Z^{-1}\left(\omega_{k}\right)-2 \varepsilon Q \omega_{k}^{2 Q-1}(\tau)\left|\omega_{k}\right|_{C_{\beta}}^{2 Q-1} \partial_{\varphi}^{ \pm}\left|\omega_{k}\right|_{C_{\beta}} Z^{-2}\left(\omega_{k}\right), \\
\partial_{h}^{ \pm} g(\omega)=(2 Q-1)\left[\Delta \omega_{k}\left(\tau, \tau^{\prime}\right)\right]^{2 Q-2}\left[\varphi(\tau)-\varphi\left(\tau^{\prime}\right)\right] Z^{-1}\left(\omega_{k}\right) \\
-2 \varepsilon Q\left[\Delta \omega_{k}\right]^{2 Q-1}\left|\omega_{k}\right|_{C_{\beta}}^{2 Q-1} \partial_{\varphi}^{ \pm}\left|\omega_{k}\right|_{C_{\beta}} Z^{-2}\left(\omega_{k}\right)
\end{gathered}
$$

and then majorate them by

$$
\begin{gathered}
\left|\partial_{h}^{ \pm} f(\omega)\right| \leq 4 Q|\varphi|_{C_{\beta}} \omega_{k}^{2 Q-2}(\tau) Z^{-1}\left(\omega_{k}\right), \\
\left|\partial_{h}^{ \pm} g(\omega)\right| \leq 8 Q|\varphi|_{C_{\beta}}\left[\Delta \omega_{k}\right]^{2 Q-2} Z^{-1}\left(\omega_{k}\right) .
\end{gathered}
$$

Let $\mu \in \mathcal{G}_{t}=\mathcal{M}_{t}^{b}$ and $\mu\left(\Omega_{\beta}^{-p, R_{W}}\right)=1$ for some $p>d$. Since by (5.3) certainly $b_{i} \in L^{1}(\mu)$, Proposition 4.13 enables us to apply to $f, g$ the (IbP)-formula (4.57) in the directions $h:=e_{k} \otimes \varphi$ with $\varphi \in T_{\beta}:=\operatorname{lin}\left\{\varphi_{n}\right\}_{n \in \mathbb{Z}}$. Therefore, in view of (5.8) and (5.9), we get that

$$
\begin{aligned}
& \int_{\Omega} \omega_{k}^{2 Q-1}(\tau)\left(A_{\beta} \varphi, \omega_{k}\right)_{H} Z^{-1} d \mu \\
& \quad \leq|\varphi|_{C_{\beta}} \int_{\Omega}\left[4 Q \omega_{k}^{2 Q-2}(\tau)+\left|\omega_{k}(\tau)\right|^{2 Q-1}\left|F_{k}^{V, W}(\omega)\right|_{L_{\beta}^{1}}\right] Z^{-1} d \mu
\end{aligned}
$$

and

$$
\begin{aligned}
& \int_{\Omega}\left[\Delta \omega_{k}\right]^{2 Q-1}\left(A_{\beta} \varphi, \omega_{k}\right)_{H} Z^{-1} d \mu \\
& \quad \leq|\varphi|_{C_{\beta}} \int_{\Omega}\left[8 Q\left[\Delta \omega_{k}\right]^{2 Q-2}+\left|\Delta \omega_{k}\right|^{2 Q-1}\left|F_{k}^{V, W}(\omega)\right|_{L_{\beta}^{1}}\right] Z^{-1} d \mu .
\end{aligned}
$$

Moreover, due to the conditions $\left|\omega_{k}\right|_{L_{\beta}^{2}},\left|F_{k}^{V, W}(\omega)\right|_{L_{\beta}^{1}} \in L^{1}(\mu)$ imposed in $(\mathbf{H})$, both these inequalities extend by continuity to all $\varphi \in \mathcal{D}\left(A_{\beta}\right)$.

(ii) Now we proceed analogously to the proof of Proposition 4.13. Namely, using the Yosida approximation of the Green function $\mathfrak{G}_{\tau}=A_{\beta}^{-1} \delta_{\tau}$ by $\left(\varphi_{\tau}^{(K)}\right)_{K \in \mathbb{N}} \subset \mathcal{D}\left(A_{\beta}\right)$ with 
the properties (4.75)-(4.77), by Lebesgue's dominated convergence theorem we conclude from (5.10) written for $\varphi^{(K)}:=\varphi_{\tau}^{(K)}$ that

$$
\int_{\Omega} \omega_{k}^{2 Q}(\tau) Z^{-1} d \mu \leq\left|\mathfrak{G}_{\tau}\right|_{C_{\beta}} \int_{\Omega}\left[4 Q \omega_{k}^{2 Q-2}(\tau)+\left|\omega_{k}(\tau)\right|^{2 Q-1}\left|F_{k}^{V, W}(\omega)\right|_{L_{\beta}^{1}}\right] Z^{-1} d \mu
$$

Recall in this respect that by (3.30) and (3.31),

$$
\sup _{\tau \in S_{\beta}}\left|\mathfrak{G}_{\tau}\right|_{C_{\beta}} \leq \mathfrak{g}:=\left[2 a \sqrt{\mathfrak{m}}\left(1-e^{-\frac{a}{\sqrt{\mathfrak{m}}} \beta}\right)\right]^{-1} .
$$

Therefore, by Hölder's inequality applied to the RHS of (5.12),

$$
\left(\int_{\Omega} \omega_{k}^{2 Q}(\tau) Z^{-1} d \mu\right)^{1 / Q} \leq \mathfrak{g}\left[4 Q+\left(\int_{\Omega} \omega_{k}^{2 Q}(\tau) Z^{-1} d \mu\right)^{1 / 2 Q}\left(\int_{\Omega}\left|F_{k}^{V, W}\right|_{L_{\beta}^{1}}^{2 Q} d \mu\right)^{1 / 2 Q}\right]
$$

which obviously yields

$$
\int_{\Omega} \omega_{k}^{2 Q}(\tau) Z^{-1} d \mu \leq C_{2 Q}:=(4 \mathfrak{g})^{Q}\left[(4 Q)^{Q}+\mathfrak{g}^{Q} c_{2 Q}(F)\right]
$$

Letting $\varepsilon \searrow 0$ in (5.13), from Fatou's lemma we readily obtain the required estimate (5.4).

(iii) In a similar way, taking the approximation of $\mathfrak{G}_{\tau}-\mathfrak{G}_{\tau^{\prime}}$ by $\varphi_{\tau, \tau^{\prime}}^{(K)}:=\varphi_{\tau}^{(K)}-\varphi_{\tau^{\prime}}^{(K)}$, we conclude from (5.11) that

$$
\begin{aligned}
& \int_{\Omega}\left[\Delta \omega_{k}\right]^{2 Q} Z^{-1} d \mu \\
& \quad \leq\left|\mathfrak{G}_{\tau}-\mathfrak{G}_{\tau^{\prime}}\right|_{C_{\beta}} \int_{\Omega}\left[8 Q\left[\Delta \omega_{k}\right]^{Q-2}+\left|\Delta \omega_{k}\left(\tau, \tau^{\prime}\right)\right|^{Q-1}\left|F_{k}^{V, W}(\omega)\right|_{L_{\beta}^{1}}\right] Z^{-1} d \mu .
\end{aligned}
$$

Then again by (3.31) and Hölder's inequality,

$$
\begin{aligned}
& \left(\int_{\Omega}\left[\Delta \omega_{k}\left(\tau, \tau^{\prime}\right)\right]^{2 Q} Z^{-1} d \mu\right)^{1 / Q} \\
& \quad \leq \frac{a \mathfrak{g}}{\sqrt{\mathfrak{m}}} \rho\left(\tau, \tau^{\prime}\right)\left[8 Q+\left(\int_{\Omega}\left[\Delta \omega_{k}\left(\tau, \tau^{\prime}\right)\right]^{2 Q} Z^{-1} d \mu\right)^{1 / 2 Q}\left(\int_{\Omega}\left|F_{k}^{V, W}\right|_{L_{\beta}^{1}}^{2 Q} d \mu\right)^{1 / 2 Q}\right],
\end{aligned}
$$

and thus for all $\varepsilon>0$,

$$
\int_{\Omega}\left[\Delta \omega_{k}\left(\tau, \tau^{\prime}\right)\right]^{2 Q} Z^{-1} d \mu \leq \Delta C_{2 Q} \cdot \rho^{Q}\left(\tau, \tau^{\prime}\right)
$$

with the constant

$$
\Delta C_{2 Q}:=\left(4 \mathfrak{g} \frac{a}{\sqrt{\mathfrak{m}}}\right)^{Q}\left[(8 Q)^{Q}+\left(\mathfrak{g} \frac{a \beta}{\sqrt{\mathfrak{m}}}\right)^{Q} I_{2 Q}\right] .
$$

Letting $\varepsilon \searrow 0$ in (5.15), by Fatou's lemma we obtain the required estimate (5.5). Finally we note that, according to $(5.23),(5.25)$, both $C_{2 Q}$ and $\Delta C_{2 Q}$ are linear functions of $I_{2 Q}$ and in no other way depend on $k$ and $\mu$.

Remark 5.5. (i) Except for Hypothesis $(\mathbf{H})$ on the uniform integrability of $\left|F_{k}^{V, W}(\omega)\right|_{L_{\beta}^{1}}^{Q}$ w.r.t. $\mu \in \mathcal{G}_{t}$, no more assumptions on the interaction potentials (such as, e.g., $(\mathbf{V}),(\mathbf{J})$, (W) in Definition 3.2) are at all needed for the proof of Lemma 5.4. In fact, the result is completely determined by the regularity properties of the Green function $\mathfrak{G}_{\tau}$ of the elliptic operator $A_{\beta}$ in the Hilbert space $H:=L_{\beta}$. 
Having obtained for $\mu \in \mathcal{G}_{t}$ the estimates from Lemma 5.4, we are ready to prove our Main Theorem II previously announced in Subsect. 2.3.

Corollary 5.6. Let Hypothesis $(\mathbf{H})$ be fulfilled. Then the set $\mathcal{G}_{t}$ of all tempered Euclidean Gibbs measures has the following properties:

(i) (cf. Theorem II) The uniform a priori bound (2.18), i.e.,

$$
\sup _{\mu \in \mathcal{G}_{t}} \sup _{k \in \mathbb{Z}^{d}} \int_{\Omega}\left|\omega_{k}\right|_{C_{\beta}^{\alpha}}^{Q} d \mu(\omega) \leq C_{Q, \alpha}<\infty, \quad \forall Q \geq 1, \alpha \in\left[0, \frac{1}{2}\right),
$$

holds for all $\mu \in \mathcal{G}_{t}$, where by the definition $\mathcal{G}_{t}:=\mathcal{G}_{(s) t}^{R}$ for the general $Q L S$ model (3.1) and respectively $\mathcal{G}_{t}:=\mathcal{G}_{(e) t}^{R}\left(\supseteq \mathcal{G}_{(s) t}^{R}\right)$ for the particular QLS models I-III (with the pair interaction satisfying the stronger decay Assumption $\left.\left(\mathbf{J}_{\mathbf{0}}\right)(\mathrm{ii})\right)$.

(ii) (cf. Corollary after Theorem II) Every $\mu \in \mathcal{G}_{t}$ is supported by

$$
\Omega_{\text {supp }}:=\bigcap_{0 \leq \alpha<1 / 2, p>d} \mathcal{C}_{-p}^{\alpha} \subset \bigcap_{0 \leq \alpha<1 / 2}\left[C_{\beta}^{\alpha}\right]^{\mathbb{Z}^{d}} \subset \Omega,
$$

where, recalling the definition (3.13),

$$
\mathcal{C}_{-p}^{\alpha}:=l^{2}\left(\gamma_{-p} ; C_{\beta}^{\alpha}\right) \text { with }\|\omega\|_{-p, \alpha}^{2}:=\sum_{k \in \mathbb{Z}^{d}}(1+|k|)^{-2 p}\left|\omega_{k}\right|_{C_{\beta}^{\alpha}}^{2}<\infty .
$$

Moreover, the set $\mathcal{G}_{t}$ is compact w.r.t. the topology of weak convergence of measures on any Banach space $\mathcal{C}_{-p}^{\alpha}$ with $p>2 d$ and $0 \leq \alpha<1 / 2$. For the particular $Q L S$ models I, II (namely, those with local interaction), $\mathcal{G}_{t}$ is even compact w.r.t. the topology of weak convergence of measures on any of product spaces $\left[C_{\beta}^{\alpha}\right]^{\mathbb{Z}^{d}}$ with $0 \leq \alpha<1 / 2$ (these latter are considered as the Fréchet spaces equipped with the system of norms $\left.\left|\omega_{k}\right|_{C_{\beta}^{\alpha}}, k \in \mathbb{Z}^{d}\right)$.

Proof. (i) We employ a standard argument related to Kolmogorov's continuity criterion. More precisely, using inequality (3.d) in BY82 (which in turn is a consequence of the well-known Garsia-Rodemich-Rumsey lemma), one can deduce from (5.5) that

$$
\int_{\Omega} \sup _{\tau \neq \tau^{\prime} \in S_{\beta}}\left[\frac{\left|\omega_{k}(\tau)-\omega_{k}\left(\tau^{\prime}\right)\right|}{\rho^{\alpha}\left(\tau, \tau^{\prime}\right)}\right]^{2 Q} d \mu(\omega) \leq C_{2 Q, \alpha}^{\prime}<\infty
$$

for all $Q>1$ and $\alpha \in\left[0, \frac{1}{2}-\frac{1}{2 Q}\right)$. Here $C_{2 Q, \alpha}^{\prime}$ is some universal constant, which is the same for all measures on $\Omega$ satisfying (5.5) and can be calculated explicitly (cf. BY82]). Letting $Q \rightarrow \infty$, both (5.4) and (5.18) give us the required bound (2.18), namely that

$$
\sup _{\mu \in \mathcal{G}_{t}} \sup _{k \in \mathbb{Z}^{d}} \int_{\Omega}\left|\omega_{k}\right|_{C_{\beta}^{\alpha}}^{Q} d \mu(\omega) \leq C_{Q, \alpha}<\infty, \text { for all } Q \geq 1, \alpha \in\left[0, \frac{1}{2}\right) .
$$

(ii) The support property (5.17) follows immediately from (2.18) by the estimate

$$
\sup _{\mu \in \mathcal{G}_{t}} E_{\mu}\|\omega\|_{-p, \alpha} \leq\left(\sup _{\mu \in \mathcal{G}_{t}} \sup _{k \in \mathbb{Z}^{d}} E_{\mu}\left|\omega_{k}\right|_{C_{\beta}^{\alpha}}^{2}\right)^{1 / 2} \sum_{k \in \mathbb{Z}^{d}}(1+|k|)^{-2 p}<\infty .
$$

On the other hand, taking into account (3.4), it is easy to check that the embedding $\mathcal{C}_{-p^{\prime}}^{\alpha^{\prime}} \subset \mathcal{C}_{-p}^{\alpha}$ is compact as soon as $\alpha^{\prime}>\alpha$ and $p>p^{\prime}$. Thus by Prokhorov's criterion we conclude from (5.19) that the family $\mathcal{G}_{t}$ is tight w.r.t. the topology of weak convergence in all $\mathcal{C}_{-p}^{\alpha}$ with $p>d$, and hence in all $\left[C_{\beta}^{\alpha}\right]^{\mathbb{Z}^{d}}$ with $0 \leq \alpha<1 / 2$ as well. Finally, $\mathcal{G}_{t}$ is closed in the above topologies according to Remark 3.12 (iii). 
5.2.2. Existence of Euclidean Gibbs measures. Respectively applying Lemma 5.4 to the probability kernels $\pi_{\Lambda}(d \omega \mid \xi)$ of the local specification $\left(\pi_{\Lambda}\right)_{\Lambda \Subset \mathbb{Z}^{d}}$, one gets for them the following Kolmogorov type estimates:

Lemma 5.7. For given $\Lambda \subseteq \mathbb{Z}^{d}$ and boundary condition $\xi \in \Omega_{t}$, let the measure $\pi_{\Lambda}(d \omega \mid \xi)$ obey the integrability property

$$
\int_{\Omega}\left[\left|\omega_{k}\right|_{L_{\beta}^{2}}+\left|F_{k}^{V, W}(\omega)\right|_{L_{\beta}^{1}}\right]^{2 Q} \pi_{\Lambda}(d \omega \mid \xi) \leq I_{2 Q, k}, \quad k \in \Lambda,
$$

with some $Q \in \mathbb{N}$ and corresponding $I_{2 Q, k} \in(0, \infty)$. Then (5.20) implies the moment estimates for all $\tau, \tau^{\prime} \in S_{\beta}$,

$$
\begin{gathered}
\sup _{\tau \in S_{\beta}} \int_{\Omega} \omega_{k}^{2 Q}(\tau) \pi_{\Lambda}(d \omega \mid \xi) \leq H_{2 Q}\left(1+I_{2 Q, k}\right)=: C_{2 Q}, \\
\int_{\Omega}\left[\omega_{k}(\tau)-\omega_{k}\left(\tau^{\prime}\right)\right]^{2 Q} \pi_{\Lambda}(d \omega \mid \xi) \leq \Delta H_{2 Q}\left(1+I_{2 Q, k}\right) \cdot \rho^{Q}\left(\tau, \tau^{\prime}\right)=: \Delta C_{2 Q}
\end{gathered}
$$

with absolute (i.e., independent of $k, \Lambda$ and $\xi$ ) constants $H_{2 Q}, \Delta H_{2 Q} \in(0, \infty)$.

From Lemma 5.7 one standardly derives a priori bounds in the spin spaces $C_{\beta}^{\alpha}$ :

Corollary 5.8. Under the assumptions of Lemma 5.7, for all $\alpha \in\left[0, \frac{1}{2}-\frac{1}{2 Q}\right)$,

$$
\int_{\Omega}\left|\omega_{k}\right|_{C_{\beta}^{\alpha}}^{2 Q} \pi_{\Lambda}(d \omega \mid \xi) \leq H_{2 Q, \alpha}\left(1+I_{2 Q, k}\right)=: C_{2 Q, \alpha}<\infty
$$

holds with an absolute (i.e., independent of $k, \Lambda$ and $\xi)$ constant $H_{2 Q, \alpha} \in(0, \infty)$.

Proof. By arguments similar to those used in the proof of Corollary 5.6, the above estimates $(5.21),(5.22)$ yield that for any $\alpha \in\left[0, \frac{1}{2}-\frac{1}{2 Q}\right)$ :

$$
\int_{\Omega} \sup _{\tau \neq \tau^{\prime} \in S_{\beta}}\left[\frac{\left|\omega_{k}(\tau)-\omega_{k}\left(\tau^{\prime}\right)\right|}{\rho^{\alpha}\left(\tau, \tau^{\prime}\right)}\right]^{2 Q} \pi_{\Lambda}(d \omega \mid \xi) \leq C_{2 Q, \alpha}^{\prime}<\infty .
$$

An important point here is that (as was calculated in $\mathrm{BY} 82$ ) the constant $C_{2 Q, \alpha}^{\prime}$ in (5.24) linearly depends on $\Delta C_{2 Q}$ in the RHS in (5.22). Hence, in view of Lemma 5.7, the desired estimate (5.23) holds with the constant $C_{2 Q, \alpha}$ which linearly depends on $I_{2 Q}$ in the RHS in (5.20).

In turn, Corollary 5.8 now readily implies the existence of $\mu \in \mathcal{G}_{t}$ as was announced in our Main Theorem I in Subsect. 2.3.

Corollary 5.9. Let Hypothesis $\left(\mathbf{H}_{l o c}\right)$ be fulfilled for some $Q>1$ and a given boundary condition $\xi \in \Omega_{t}$ satisfying

$$
\xi \in\left[C_{\beta}^{\alpha}\right]^{\mathbb{Z}^{d}} \text { and } \sup _{k \in \mathbb{Z}^{d}}\left|\xi_{k}\right|_{C_{\beta}^{\alpha}}<\infty
$$

for some $\alpha:=\alpha(\xi)>0$. Then the following statements hold:

(i) (cf. a priori estimate (2.19) in Theorem III) For all $0 \leq \alpha^{\prime}<\min \left\{\alpha, \frac{1}{2}-\frac{1}{2 Q}\right\}$

$$
\sup _{\Lambda \Subset \mathbb{Z}^{d}} \sup _{k \in \mathbb{Z}^{d}} \int_{\Omega}\left|\omega_{k}\right|_{C_{\beta}^{\alpha^{\prime}}}^{2 Q} \pi_{\Lambda}(d \omega \mid \xi)<\infty
$$

and hence the family $\left\{\pi_{\Lambda}(d \omega \mid \xi)\right\}_{\Lambda \Subset \mathbb{Z}^{d}}$ is tight in all spaces $\mathcal{C}_{-p}^{\alpha^{\prime}}$ with $p>d$.

(ii) (Existence of $\mu \in \mathcal{G}_{t}$; cf. Theorem I) The set $\mathcal{G}_{t}$ is not empty as such containing each accumulation point as $\Lambda \nearrow \mathbb{Z}^{d}$ for $\left\{\pi_{\Lambda}(d \omega \mid \xi)\right\}_{\Lambda \Subset \mathbb{Z}^{d}}$. 
Proof. (i) (5.26) follows immediately from (5.24) due to the special choice (5.22) of the boundary condition $\xi$. On the other hand, by (3.4) and the definition (3.13) of the Banach spaces $\mathcal{C}_{-p}^{\alpha}:=l^{2}\left(\gamma_{-p} ; C_{\beta}^{\alpha}\right)$ it is easy to check that the embedding $\mathcal{C}_{-p^{\prime}}^{\alpha^{\prime}} \subset \mathcal{C}_{-p}^{\alpha}$ is compact as soon as $\alpha^{\prime}>\alpha$ and $p>p^{\prime}$. Thus by Prokhorov's criterion we conclude from (5.26) that the family of distributions $\pi_{\Lambda^{(K)}}(d \omega \mid \xi)$, where $\Lambda^{(K)} \nearrow \mathbb{Z}^{d}$ as $K \rightarrow \infty$, is tight in all $\mathcal{C}_{-p}^{\alpha}$ with $p>d$ and $\alpha \in\left[0, \frac{1}{2}-\frac{1}{2 Q}\right)$.

(ii) By (i) there exists a subsequence $\pi_{\Lambda^{\left(K_{L}\right)}}(d \omega \mid \xi), L \in \mathbb{N}$, which converges weakly to some probability measure $\mu_{*}$ on $\mathcal{C}_{-p}^{\alpha}$. Since $\mathcal{C}_{-p}^{\alpha}$ is continuously embedded into $\Omega_{-p}^{R}$, this subsequence converges to $\mu_{*}$ also weakly on all Polish spaces $\Omega_{-p}^{R}$ with $p>2 d$. This means by Proposition 5.3 that $\mu_{*} \in \mathcal{G}_{t}:=\mathcal{G}_{(s) t}^{R}$.

Next, we analyze in more detail the dependence on a boundary condition $\xi \in \Omega_{t}$ in the a priori estimates for $\left\{\pi_{\Lambda}(d \omega \mid \xi)\right\}_{\Lambda \Subset \mathbb{Z}^{d}}$.

Corollary 5.10. (i) Let $\xi \in \Omega_{t}:=\Omega_{(s) t}^{R}$ and hence (in the notation of Subsect. 3.2.3)

$$
\|\xi\|_{\mathcal{L}_{-p}^{R}}^{2}:=\sum_{k \in \mathbb{Z}^{d}}(1+|k|)^{-p}\left|\xi_{k}\right|_{L_{\beta}^{R}}^{2}<\infty \quad \text { for some } p=p(\xi)>d .
$$

For the general $Q L S$ model, (3.1) then holds for $Q \geq 1, p^{\prime}>p R Q+d$ and $\alpha \in\left[0, \frac{1}{2}-\frac{1}{Q}\right)$ :

$$
\sup _{\Lambda \Subset \mathbb{Z}^{d}} \sum_{k \in \Lambda}(1+|k|)^{-p^{\prime}} \int_{\Omega}\left|\omega_{k}\right|_{C_{\beta}^{\alpha}}^{Q} \pi_{\Lambda}(d \omega \mid \xi)<\infty .
$$

(ii) (cf. Estimate (2.20) in Theorem III) Let $\xi \in \Omega_{t}:=\Omega_{(e) t}^{R}$ and hence

$$
\|\xi\|_{\mathcal{L}_{-\delta}^{R}}^{2}:=\sum_{k \in \mathbb{Z}^{d}} \exp (-\delta|k|)\left|\xi_{k}\right|_{L_{\beta}^{R}}^{2}<\infty \quad \text { for all } \delta>0 .
$$

For the particular QLS models I, II (and also for the QLS model III satisfying additionally Assumption ( $\left.\boldsymbol{J}_{\mathbf{0}}\right)$ (ii)), (3.1) then holds for all $Q \geq 1, \delta>0$ and $\alpha \in\left[0, \frac{1}{2}\right)$ :

$$
\sup _{\Lambda \Subset \mathbb{Z}^{d}} \sum_{k \in \Lambda} \exp (-\delta|k|) \int_{\Omega}\left|\omega_{k}\right|_{C_{\beta}^{\alpha}}^{Q} \pi_{\Lambda}(d \omega \mid \xi)<\infty .
$$

Proof. The proof of both statements (i) and (ii) follows the same line. Namely, with regard to Corollary 5.8, it suffices to check that (5.27), resp. (5.29), implies that for all $Q \in \mathbf{N}$ and $k \in \mathbb{Z}^{d}$,

$$
\sup _{\Lambda \Subset \mathbb{Z}^{d} \backslash\{k\}} \int_{\Omega}\left[\left|\omega_{k}\right|_{L_{\beta}^{2}}+\left|F_{k}^{V, W}(\omega)\right|_{L_{\beta}^{1}}\right]^{Q} \pi_{\Lambda}(d \omega \mid \xi):=I_{Q, k}
$$

with a nonnegative sequence $I_{Q}=\left(I_{Q, k}\right)_{k \in \mathbb{Z}^{d}}$ such that for all $p^{\prime}>p R Q+d$,

$$
\sum_{k \in \mathbb{Z}^{d}}(1+|k|)^{-p^{\prime}} I_{Q, k}<\infty
$$

or respectively for all $\delta>0$,

$$
\sum_{k \in \Lambda} \exp (-\delta|k|) I_{Q, k}<\infty
$$

The verification of these key conjectures (together with Hypotheses $(\mathrm{H})$ and $\left(\mathrm{H}_{l o c}\right)$ ) will be performed in Subsect. 7.3. 
Remark 5.11. (i) For the translation invariant systems, by an obvious modification of the arguments used above one can construct the so-called periodic Euclidean Gibbs measures $\mu_{\text {per }} \in \mathcal{G}_{t}$. They are defined as accumulation points for the family $\mu_{\mathfrak{T}(\Lambda)}, \Lambda \Subset \mathbb{Z}^{d}$, of local Gibbs distributions with periodic boundary conditions and, hence, are certainly $\mathbb{Z}_{0}^{d}$-translation invariant (cf. the related discussion in Remark 3.10 (iv)). We emphasize that in doing so the crucial estimate

$$
\sup _{\Lambda \Subset \mathbb{Z}^{d}} \sup _{k \in \mathbb{Z}^{d}} \int_{\Omega_{\Lambda}}\left|\omega_{k}\right|_{C_{\beta}^{\alpha}}^{2 Q} \mu_{\mathfrak{T}(\Lambda)}\left(d \omega_{\Lambda}\right)<\infty
$$

holds, provided we assume the following Hypothesis to be satisfied:

$\left(\mathbf{H}_{\text {per }}\right)$ For some fixed $Q>1$,

$$
I_{2 Q, p e r}(F):=\sup _{\Lambda \Subset \mathbb{Z}^{d}} \sup _{k \in \Lambda} \int_{\Omega_{\Lambda}}\left[\left|\omega_{k}\right|_{L_{\beta}^{2}}+\left|F_{k}^{V, W}(\omega)\right|_{L_{\beta}^{1}}\right]^{2 Q} \mu_{\mathfrak{T}(\Lambda)}\left(d \omega_{\Lambda}\right)<\infty .
$$

(ii) (Existence of Superstable Gibbs States) As already mentioned in Remark 2.4 (ii), $\mathcal{G}_{t}:=\mathcal{G}_{(s) t}^{R}$ contains a class $\mathcal{G}_{(s s) t}$ of the so-called Ruelle type "superstable" Gibbs measures, which for the considered quantum lattice systems (in the particular case $R=2$ only) has been introduced in [PaY94] by the support condition

$$
\begin{gathered}
\mathcal{G}_{(s s) t}:=\left\{\mu \in \mathcal{G} \mid \mu\left(\Omega_{(s s) t}\right)=1\right\}, \\
\Omega_{(s s) t}:=\left\{\omega \in \Omega \mid \sup _{n \in \mathbb{N}}\left[(1+2 n)^{-d} \sum_{|k| \leq n}\left|\omega_{k}\right|_{L_{\beta}^{2}}^{2}\right]<\infty\right\} .
\end{gathered}
$$

But for any measure $\mu \in \mathcal{M}(\Omega)$, which is translation invariant and satisfies the a priori estimates (2.18), the support condition (even much stronger than (5.35)) holds for all $Q \geq 1$ and $\alpha \in[0,1 / 2)$, namely:

$$
\left.\sup _{n \in \mathbb{N}}\left\{(1+2 n)^{-d} \sum_{|k| \leq n}\left|\omega_{k}\right|_{C_{\beta}^{\alpha}}^{Q}\right\} \leq C_{Q, \alpha}(\omega)<\infty, \quad \text { for all } \omega \in \Omega \text { ( } \mu \text {-a.e. }\right) .
$$

The latter follows from the Birkhoff-Khinchin ergodic theorem (cf. e.g. [DeuS89]) applied to the stationary process $\omega_{k}, k \in \mathbb{Z}^{d}$, on the probability space $(\Omega, \mu)$. Together with (i) this means that we can refine the statement of Theorem I by claiming the existence of $\mu_{\text {per }} \in \mathcal{G}_{(s s) t}$ with the additional support property (5.36).

5.3. Comparison with the stochastic dynamics method. Here we would like to point out some advantages of our approach in comparison to the stochastic dynamics employed in AKRT01 to the quantum lattice models (2.1).

In that paper we have restricted ourselves to the case of harmonic pair interactions (described by a dynamical matrix $0 \leq \mathbf{D}:=\left(a_{k, j}\right)_{k, j \in \mathbb{Z}^{d}} \in \mathcal{L}\left(l^{2}\left(\mathbb{Z}^{d}\right)\right)$, see Remark 3.3 (iv)); in more generality the method could be applied to many-particle interactions of at most quadratic growth. Concerning the one-particle potentials $V_{k} \in C_{b, l o c}^{2}(\mathbb{R} \rightarrow \mathbb{R})$, the following semi-monotonicity

$$
\left(V_{k}^{\prime}\left(q_{1}\right)-V_{k}^{\prime}\left(q_{2}\right)\right)\left(q_{1}-q_{2}\right) \geq K_{5}^{-1}\left(q_{1}-q_{2}\right)^{2}-L_{5}
$$

and at most polynomial growth

$$
\left|V_{k}^{\prime}\left(q_{1}\right)\right| \leq K_{6}\left(1+\left|q_{1}\right|\right)^{R}
$$

conditions with some fixed $K_{5}, K_{6}, L_{5}>0$ and $R \geq 1$ are required to hold uniformly for all $k \in \mathbb{Z}^{d}$ and $q_{1}, q_{2} \in \mathbb{R}$. (Of course, our assumptions ( $\mathbf{V}_{\text {i-iv }}$ ) do not imply by themselves (5.63) and (5.64)). 
So, in AKRT01] we have firstly constructed a Markov process $x_{t}=\left(x_{k, t}\right)_{k \in \mathbb{Z}^{d}}, t \geq$ 0, which gives the unique (generalized) solution to the (so-called Langevin) stochastic evolution equation with a drift term being the logarithmic gradient $b=\left(b_{k}\right)_{k \in \mathbb{Z}^{d}}$ of the measures $\mu \in \mathcal{G}_{t}$. More precisely, $x_{t}, t \geq 0$, takes values in the Banach state space $X:=\mathcal{C}_{-p}=l^{2}\left(\mathbb{Z}^{d} \rightarrow C_{\beta} ; \gamma_{-p}\right) \subset \Omega$ (with large enough $p=p(R)>d$ ) and satisfies the following infinite system of stochastic partial differential equations (SPDE's):

$$
\left\{\begin{array}{c}
\frac{\partial}{\partial t} x_{k, t}=-\frac{1}{2}\left[A_{\beta} x_{k, t}+\sum_{j \in \mathbb{Z}^{d}} a_{k, j} x_{j, t}+V_{k}^{\prime}\left(x_{k, t}\right)\right]+\dot{w}_{k, t}, \\
k \in \mathbb{Z}^{d} \quad\left(t>0, \tau \in S_{\beta}\right) .
\end{array}\right.
$$

Here $A_{\beta}=-\mathfrak{m} \partial^{2} / \partial \tau^{2}+a^{2} \mathbf{1}$ is the selfadjoint operator in $L_{\beta}^{2}$ introduced in Subsect. 3.3.1 and $\dot{w}_{k, t}(\tau)$ is a Gaussian white noise on $\Omega \times[0, \infty)$ (heuristically, $E \dot{w}_{k_{1}, t_{1}}\left(\tau_{1}\right) \times \dot{w}_{k_{2}, t_{2}}\left(\tau_{2}\right)$ $=\delta_{k_{1}-k_{2}} \delta_{t_{1}-t_{2}} \delta_{\tau_{1}-\tau_{2}}$ ). In the trivial case when $\mathbf{D}=0$ and $V=0$, the solution of (5.39), starting with initial data $g_{0}:=\zeta \in \mathcal{C}_{-p}$, is explicitly given by the Ornstein-Uhlenbeck process $g_{t}=\left(g_{k, t}\right)_{k \in \mathbb{Z}^{d}}, t \geq 0$,

$$
g_{k, t}:=e^{-t A_{\beta} / 2} \zeta_{k}+\int_{0}^{t} e^{(t-s) A_{\beta} / 2} d w_{k, s}, \quad k \in \mathbb{Z}^{d}, t \geq 0 .
$$

Taking into account the regularity properties (3.31) of the Green function $\mathfrak{G}_{\tau}=A_{\beta}^{-1} \delta_{\tau}$, one can deduce from (5.40) that $g_{t}, t \geq 0$, possesses a continuous modification in the spaces of Hölder loops $\mathcal{C}_{-p}^{\alpha}, p>d, 0<\alpha<1 / 2$, and its polynomial moments are ultimately bounded, i.e., $\forall Q \geq 1$,

$$
\sup _{g_{0} \in \mathcal{C}_{-p}} \sup _{k \in \mathbb{Z}^{d}} \limsup _{t \rightarrow \infty} E\left|g_{k, t}\right|_{C_{\beta}^{\alpha}}^{Q}<\infty .
$$

Moreover, $g_{t}, t \geq 0$, is ergodic with the unique invariant (and also reversible) distribution $\gamma_{\text {inv }}(d \omega):=\prod_{k \in \mathbb{Z}^{d}} \gamma_{\beta}\left(d \omega_{k}\right)$; i.e., the corresponding laws $\mathcal{L}\left(g_{t}\right)$ converge weakly, as $t \rightarrow$ $\infty$, to $\gamma_{\text {inv }}$. So it is reasonable to compare the solution $x_{t}, t \geq 0$, of the nonlinear problem (5.39) with the Gaussian process $g_{t}, t \geq 0$. If their initial values coincide, i.e., $x_{0}=g_{0}:=\zeta$, then for the deviation process

$$
y_{k, t}=x_{k, t}-g_{k, t}=\int_{0}^{t} e^{(t-s) A_{\beta} / 2}\left(\sum_{j \in \mathbb{Z}^{d}} a_{k, j} x_{j, s}+V_{k}^{\prime}\left(x_{k, s}\right)\right) d s
$$

some helpful energy estimates hold. So, under the above assumptions (5.37) and (5.38), for all $Q \geq 1$ :

$$
\begin{array}{r}
\sup _{\substack{x_{0} \in \mathcal{C}-p \\
x_{0}=g_{0}}} \sup _{k \in \mathbb{Z}^{d}} \limsup _{t \rightarrow \infty} E\left\|y_{k, t}\right\|_{C_{\beta}}^{Q}<\infty, \\
\sup _{\substack{x_{0} \in \mathcal{C} \\
x_{0}=g_{0}}} \sup _{k \in \mathbb{Z}^{d}} \limsup _{t \rightarrow \infty} \frac{1}{t} \int_{t}^{2 t} E\left\|y_{k, s}\right\|_{W_{\beta}^{2,1}}^{Q} d s<\infty .
\end{array}
$$

Combined, (5.41) and (5.43) give us a crucial estimate for the process $x_{t}, t \geq 0$, that we shall use in the sequel:

$$
\sup _{x_{0} \in \mathcal{C}} \sup _{-p} \limsup _{k \in \mathbb{Z}^{d}} \frac{1}{t} \int_{t \rightarrow \infty}^{2 t} E\left\|x_{k, s}\right\|_{C_{\beta}^{\alpha}}^{Q} d s:=I_{Q, \alpha}^{\prime}<\infty .
$$

As was further shown in AKRT01,

$$
\left(\mathbb{P}_{t} f\right)(\omega):=E\left\{f\left(x_{t}\right) \mid x_{0}=\omega\right\}, \quad \omega \in \mathcal{C}_{\beta}^{-p},
$$

is a Feller transition semigroup $\mathbb{P}_{t}, t \geq 0$, in the space $C_{b}\left(\mathcal{C}_{-p}\right)$ of all bounded continuous functions $f: \mathcal{C}_{-p} \rightarrow \mathbb{R}$. Let $\mathcal{R}\left(\mathcal{C}_{-p}\right)$, resp. $\mathcal{I}\left(\mathcal{C}_{-p}\right)$, denote the family of all reversible, 
resp. invariant, distributions for the Markov process $x_{t}, t \geq 0$. Then the following basic relation between Gibbs and reversible distributions is true:

$$
\mu \in \mathcal{G} \quad \text { and } \quad \mu\left(\mathcal{C}_{-p}\right)=1 \Longleftrightarrow \mu \in \mathcal{R}\left(\mathcal{C}_{-p}\right) \subseteq \mathcal{I}\left(\mathcal{C}_{-p}\right)
$$

(for the proof of (5.45) involving the Ito stochastic calculus and (IbP)-formulas, cf. Fu91, KRZ96). Moreover, in our situation one can directly verify (cf. e.g. Fu91) that the finite volume Gibbs measures $\mu_{\Lambda}(d \omega \mid \xi), \Lambda \Subset \mathbb{Z}^{d}, \xi \in \mathcal{C}_{-p}$, are exactly the reversible distributions for the corresponding cut-off dynamics $x_{t}^{\Lambda}=\left(x_{k, t}^{\Lambda}\right)_{k \in \Lambda}$ in $C_{\beta}^{\Lambda}$,

$$
\left\{\begin{array}{c}
\frac{\partial}{\partial t} x_{k, t}^{\Lambda}=-\frac{1}{2}\left[A_{\beta} x_{k, t}^{\Lambda}+\sum_{j \in \Lambda} a_{k, j} x_{j, t}^{\Lambda}+V_{k}^{\prime}\left(x_{k, t}^{\Lambda}\right)+\sum_{j \in \Lambda^{c}} a_{k, j} \xi_{j, t}^{\Lambda}\right]+\dot{w}_{k, t}, \\
k \in \Lambda \quad\left(t>0, \tau \in S_{\beta}\right)
\end{array}\right.
$$

Let us fix some boundary and initial conditions $\xi, \zeta \in \mathcal{C}_{-p}$. An important point is that the analogues of (5.43) and (5.44) hold uniformly in $\Lambda \Subset \mathbb{Z}^{d}$ for the corresponding solutions $x_{t}^{\Lambda}, t \geq 0$, starting from $x_{k, 0}^{\Lambda}=\zeta_{k}, k \in \Lambda$, e.g.,

$$
\sup _{\Lambda \in \mathbb{Z}^{d}, k \in \Lambda} \sup _{t \geq 0} \frac{1}{t} \int_{t}^{2 t} E\left|x_{k, s}^{\Lambda}\right|_{C_{\beta}^{\alpha}}^{2} d s:=I_{Q, \alpha}^{\prime}(\xi, \zeta)<\infty .
$$

Thus, in order to get the required information on $\mu \in \mathcal{G}_{t} \subseteq \mathcal{I}\left(\mathcal{C}_{-p}\right)$, one could apply standard tools used in the theory of SDE's for the long-time analysis of diffusion processes. So, by the ergodic theorem for invariant distributions, (5.47) implies immediately that

$$
\sup _{\substack{\mu \in \mathcal{I}\left(\mathcal{C}_{-p}\right) \\ k \in \mathbb{Z}^{d}}} \int_{\Omega}\left|\omega_{k}\right|_{C_{\beta}^{\alpha}}^{Q} d \mu(\omega) \leq \sup _{\substack{x_{0} \in \mathcal{C}_{-p} \\ k \in \mathbb{Z}^{d}}} \limsup _{t \rightarrow \infty} E\left|x_{k, t}\right|_{C_{\beta}^{\alpha}}^{Q}=I_{Q, \alpha}^{\prime}<\infty,
$$

which confirms the corresponding result in Theorem II in this special situation. Moreover, the existence of invariant measures $\mu \in \mathcal{I}\left(\mathcal{C}_{-p}\right)$ is an easy consequence of $(5.47)$ and the usual Bogolyubov-Krylov argument. For the existence of $\mu \in \mathcal{G}=\mathcal{R}\left(\mathcal{C}_{-p}\right)$ stated in Theorem I, by Proposition 5.3 it suffices to prove the tightness in $\mathcal{C}_{-p}$ of the family $\left\{\pi_{\Lambda}(d \omega \mid 0)\right\}_{\Lambda \Subset \mathbb{Z}^{d}}$ of local Gibbs distributions with fixed boundary condition $\xi=0$. By Prokhorov's criterion this is a consequence of the uniform boundedness of their moments in $\mathcal{C}_{-p}^{\alpha}, 0<\alpha<1 / 2$,

$$
\sup _{\Lambda \Subset \mathbb{Z}^{d}, k \in \Lambda} \int_{\left(C_{\beta}\right)^{\Lambda}}\left|\omega_{k}\right|_{C_{\beta}^{\alpha}}^{2} d \mu_{\beta, \Lambda}\left(\omega_{\Lambda} \mid 0\right):=I_{Q, \alpha}^{\prime}(\xi, \zeta)<\infty
$$

(see also Subsect.5.5.2). Since the finite volume dynamics (5.46) are ergodic, (5.48) immediately follows from the estimates (5.47) above.

To summarize, a disadvantage of the stochastic dynamics method is (apart from the fact that a lot of advanced techniques are involved) that the assumptions on the solvability of the related infinite-dimensional evolution equations are usually more restrictive than those under which the (IbP)-formula (4.57) makes sense. It should also be emphasized that the ergodicity problem for the interacting stochastic systems with unbounded spins like (5.30) (except for the special cases of linear or strictly dissipative ones; cf. [DPZ96, AKRT01]) is extremely difficult itself. Thus in this paper, rather than discussing the processes and their invariant (reversible) distributions, we start directly from the definition of Gibbs measures as solutions to the (IbP)-formulas.

\section{Part II: Abstract Setting}

\section{Symmetrizing measures on Banach (E.G. LOOP) SPaCeS}

In order to clarify the concept and stress the key ideas, we now put the problem in a general framework of symmetrizing measures on Banach lattices. In this section we 
discuss in detail the case of symmetrizing measures $\mu \in \mathcal{M}^{b}(X)$ on a single Banach (in particular, loop) space $X$. Assuming that the logarithmic derivative $b$ of these measures has a linear component $A$ (being a positive selfadjoint operator in some tangent space $H \supseteq X$ ) and a nonlinear component $F$ (possessing certain coercivity properties w.r.t. $H)$, we investigate the interplay between the properties of the operators $A, F$ and the integrability and support properties of $\mu$. Apart from its origin in infinite-dimensional stochastic analysis, this setting may also be of independent interest because of its possible applications to the study of time-reversible distributions of stochastic evolution equations (in particular, SPDE's of reaction-diffusion type).

6.1. (IbP)-formula in a general setting. Having in mind the concrete properties of the one-particle Euclidean distributions from Subsect.3.3.1, one can formulate an abstract setting of the problem as follows:

Let us fix a tangent space $H$ as a separable Hilbert space with inner product $(\cdot, \cdot)_{H}$ and norm $|\cdot|_{H}$. Let

$$
X \subset B \subset H \subset B^{*}
$$

be a rigging of $H$ by a locally convex vector (e.g., Banach) space $X$ and by a reflexive Banach space $B$ and its dual $B^{*}$ with (at least outside zero) differentiable norms $|\cdot|_{B}$ and $|\cdot|_{B^{*}}$ respectively. The embeddings in (6.1) are dense and continuous, which implies the relation

$$
\iota^{-1}|\cdot|_{B^{*}} \leq|\cdot|_{H} \leq \iota|\cdot|_{B}
$$

with some finite constant $\iota>0$. The duality between $B$ and $B^{*}$ is given by the inner product in $H$ and will also be denoted by $(\cdot, \cdot)_{H}$.

Let $A$ be a positive selfadjoint operator in $H$ with inverse $A^{-1}$ of finite trace. The operator $A$ has a discrete spectrum $\left\{\lambda_{n}\right\}_{n \in \mathbb{N}}$ and a complete orthonormal system of eigenvectors $\left\{\varphi_{n}\right\}_{n \in \mathbb{N}}:=\operatorname{bas}(H)$,

$$
A \varphi_{n}=\lambda_{n} \varphi_{n}, \quad \operatorname{Tr}_{H} A^{-1}=\sum_{n \in \mathbb{N}} \lambda_{n}^{-1}<\infty .
$$

Let $\mathbb{P}_{K}, K \in \mathbb{N}$, be the finite-dimensional projections generated by the first $K$ vectors of bas $(H)$, i.e.,

$$
\mathbb{P}_{K} x:=\sum_{n=1}^{K}\left(x, \varphi_{n}\right)_{H} \varphi_{n}, \quad x \in H .
$$

The following is crucial: we assume that $\varphi_{n} \in X$ for all $n \in \mathbb{N}$, and $\left\{\varphi_{n}\right\}_{n \in \mathbb{N}}$ is a Schauder basis in $B$, so that there exist finite constants $\kappa$ and $\varsigma$ such that

$$
\sup _{n \in \mathbb{N}}\left\{\left|\varphi_{n}\right|_{B}\right\} \leq \kappa, \quad\left|\mathbb{P}_{K} x\right|_{B} \leq \varsigma|x|_{B} \quad \text { for all } x \in B, K \in \mathbb{N} .
$$

In accordance with the notation in Subsect. 4.2.1, $C_{b}^{1}(X ; \varphi)$, resp. $C_{b, l o c}^{1}(X ; \varphi)$, will be the spaces of all functions $f: X \rightarrow \mathbb{R}$ which are continuous and globally, resp. locally, bounded together with their partial derivative $\partial_{\varphi} f: X \rightarrow \mathbb{R}$ along the fixed direction $\varphi \in X$. Then we define

$$
C_{b}^{1}(X):=\bigcap_{\varphi \in X} C_{b}^{1}(X ; \varphi), \quad \text { resp. } \quad C_{b, l o c}^{1}(X):=\bigcap_{\varphi \in X} C_{b, l o c}^{1}(X ; \varphi)
$$

and their subspaces $\mathcal{F} C_{b}^{1}(X)$, resp. $\mathcal{F} C_{b, l o c}^{1}(X)$, of cylinder functions w.r.t. bas $(H)$. To shorten the notation, $\partial_{n} f:=\partial_{\varphi_{n}} f$ will stand for the derivatives along the basis vectors $\varphi_{n}, n \in \mathbb{N}$. 
Furthermore, let $F: X \rightarrow B^{*}$ be a nonlinear mapping which is continuous and locally bounded together with its partial derivatives in the directions $\varphi_{n} \in \operatorname{bas}(H)$, i.e., $F \in \bigcap_{n \in \mathbb{N}} C_{b, l o c}^{1}\left(X \rightarrow B^{*} ; \varphi\right)$. We define a measurable vector field $X \ni x \rightarrow b(x):=$ $\left\{b_{n}(x)\right\}_{n \in \mathbb{N}} \in \mathbb{R}^{\mathbb{N}}$ by

$$
b_{n}(x):=-\left(A \varphi_{n}, x\right)_{H}-\left(F(x), \varphi_{n}\right)_{H}, \quad n \in \mathbb{N}, x \in X .
$$

From the above assumptions,

$$
b_{n} \in C_{b, l o c}^{1}(X) \quad \text { and } \quad\left|b_{n}(x)\right| \leq \kappa\left(\lambda_{n}|x|_{B^{*}}+|F(x)|_{B^{*}}\right) .
$$

Let $\mathcal{M}^{b}(X)$ denote the family of all Borel probability measures $\mu$ on $X$ which satisfy the following (IbP)-formula:

$$
\int_{X} \partial_{n} f d \mu=-\int_{X} f b_{n} d \mu, \quad \forall n \in \mathbb{N},
$$

on the dense subset $C_{d e c}^{1}(X) \subset C_{b}^{1}(X)$ of all functions $f$ having the following decay property:

$$
|f(x)| \leq C\left(1+|x|_{B^{*}}+|F(x)|_{B^{*}}\right)^{-1}, x \in X,
$$

with some finite $C=C(f)>0$. Obviously, both integrals in (6.8) make sense for such $f$. We will say that $\mu \in \mathcal{M}^{b}(X)$ are symmetrizing measures in the sense that they have the given logarithmic derivative $b$.

The above setup in particular applies to the single loop spaces

$$
\begin{gathered}
X=C_{\beta}, H=L_{\beta}^{2} \text { and } B=L_{\beta}^{R}, B^{*}=L_{\beta}^{R}, \\
\text { with } \quad 2<R<\infty, 1<R^{\prime}=R(R-1)^{-1}<R<\infty,
\end{gathered}
$$

and the one-particle Euclidean measure $d \sigma_{\beta}(v)$ defined in Subsect.3.3.1. The (IbP)formula (6.8) for them on the domain $C_{d e c}^{1}(X)$ with

$$
b_{n}(x)=-\left(A_{\beta} \varphi_{n}, x\right)_{H}-\left(V_{k}^{\prime}(x), \varphi_{n}\right)_{H}, \quad x \in X,
$$

follows from the flow description (3.34) as a particular case of Propositions 4.8 and 4.11 (i).

6.2. A priori integrability estimates for symmetrizing measures. In this subsection we assume that $\mathcal{M}^{b}(X) \neq \emptyset$, and derive sufficient conditions implying integrability of certain functionals (logarithmic derivatives, polynomials, higher powers of norms, etc.) w.r.t. measures $\mu \in \mathcal{M}^{b}(X)$. It should be emphasized that to get these a priori estimates we do not need to use the fact that $F$ is of gradient type. Since the existence of Euclidean Gibbs measures readily follows from the corresponding uniform integrability estimates for their local specifications (see Subsect.5.2.2), here we do not discuss at all the problem whether there exists any $\mu \in \mathcal{M}^{b}(X)$ (which is, of course, not the case for arbitrary $b$ ). Nevertheless, we note that, developing in a proper way the ideas of AKRT00, Theorem 2.3] and [BR01, Theorem 5.1], it is also possible to give an abstract existence criterion for $\mu \in \mathcal{M}^{b}(X)$ based on their approximation by finite-dimensional measures $\mu_{n}, n \in \mathbb{N}$, on $\mathbb{R}^{n}$.

6.2.1. The coercivity functional and its integrability properties. In order to study the properties of $\mu \in \mathcal{M}^{b}(X)$, conventionally in this paper we introduce the following quantity related to the vector field $F$ (i.e., the nonlinear part of the logarithmic derivative $b$ defined by (6.6)): 
Definition 6.1. The functional

$$
L_{H}^{F}: X \rightarrow \mathbb{R}, \quad L_{H}^{F}(x):=(F(x), x)_{H},
$$

will be called the coercivity functional for the vector field $F: X \rightarrow B^{*}$ w.r.t. the Hilbert space $H$.

The first and already nontrivial step is to prove the integrability of $L_{H}^{F}$ with respect to $\mu$.

Theorem 6.2 (A Priori Integrability of the Coercivity Functional). Let the following assumptions on the vector field $F: X \rightarrow B^{*}$ hold uniformly for all $x \in X$ and $n \in \mathbb{N}$ :

(F) there exist $\mathcal{K}_{0}>0$ and $\mathcal{L}_{0} \geq 0: \quad\left|\partial_{n} F(x)\right|_{B^{*}} \leq \mathcal{K}_{0}\left(|F(x)|_{B^{*}}+|x|_{B}\right)+\mathcal{L}_{0} ;$ and respectively for its coercivity functional $L_{H}^{F}: X \rightarrow \mathbb{R}$;

$\left(\mathbf{L}_{1}\right)$ there exist $\mathcal{K}_{1}>0$ and $\mathcal{L}_{1} \geq 0$ :

$\left|\left(F(x), \varphi_{n}\right)_{H}\right|+\left|\left(\partial_{n} F(x), \varphi_{n}\right)_{H}\right|+\left|\left(x, \varphi_{n}\right)_{H}\right| \leq \mathcal{K}_{1} L_{H}^{F}(x)+\mathcal{L}_{1} ;$

$\left(\mathbf{L}_{2}\right)$ there exist $\mathcal{K}_{2}>0$ and $\mathcal{L}_{2} \geq 0: \quad\left|\left(\partial_{n} F(x), x\right)_{H}\right| \leq \mathcal{K}_{2} L_{H}^{F}(x)+\mathcal{L}_{2}$.

If the above parameters satisfy the following relation for some $Q \geq 1$,

$$
\Xi_{Q-1}:=\mathcal{K}_{1}\left[1+(Q-1) \mathcal{K}_{2}\right] \operatorname{Tr}_{H} A^{-1}<1
$$

(which can be achieved, keeping fixed the other parameters in (6.11), by taking $\operatorname{Tr}_{H} A^{-1}$ or $\mathcal{K}_{1}>0$ small enough), then

$$
\sup _{\mu \in \mathcal{M}^{b}(X)} \int_{X}\left|L_{H}^{F}(x)\right|^{Q} d \mu(x) \leq \mathcal{C}_{Q}^{\prime}<\infty .
$$

Proof. To start with, note that either $\left(\mathbf{L}_{1}\right)$ or $\left(\mathbf{L}_{2}\right)$ implies the global lower boundedness of $L_{H}^{F}$, i.e., that

$$
\inf _{x \in X} L_{H}^{F}(x) \geq-l:=-\min \left\{-\mathcal{L}_{1} \mathcal{K}_{1}^{-1},-\mathcal{L}_{2} \mathcal{K}_{2}^{-1}\right\}>-\infty .
$$

We would like to apply the (IbP)-formula (6.8) along the basis vectors $\varphi_{n}$ to the following family of test functions $f \in C_{d e c}^{1}(X)$ indexed by $n \in \mathbb{N}$ and $0<\varepsilon \leq 1$ :

$$
f(x):=f_{n, \varepsilon}(x):=[\tilde{L}(x)]^{Q-1}\left(F(x), \varphi_{n}\right)_{H} Z^{-1}(x), \quad x \in X .
$$

Here, for brevity, we have introduced the notation

$$
\begin{gathered}
\varepsilon \leq \tilde{L}(x):=\tilde{L}_{\varepsilon}(x):=L_{H}^{F}(x)+l+\varepsilon, \\
1 \leq Z(x):=Z_{\varepsilon}(x):=1+\varepsilon|F(x)|_{B^{*}}^{2 Q}+\varepsilon|x|_{B}^{2 Q} .
\end{gathered}
$$

Then elementary calculations give for all $x \in X$,

$$
\begin{aligned}
\partial_{n} f_{n, \varepsilon}(x)= & {[\tilde{L}(x)]^{Q-1}\left(\partial_{n} F(x), \varphi_{n}\right)_{H} Z^{-1}(x) } \\
& +(Q-1)[\tilde{L}(x)]^{Q-2}\left[\left(\partial_{n} F(x), x\right)_{H}+\left(F(x), \varphi_{n}\right)_{H}\right]\left(F(x), \varphi_{n}\right)_{H} Z^{-1}(x) \\
& -[\tilde{L}(x)]^{Q-1}\left(F(x), \varphi_{n}\right)_{H}\left(Z^{-2} \partial_{n} Z\right)(x) .
\end{aligned}
$$

To get the upper bound on the RHS in (6.16), let us first show that

$$
\sup _{n \in N} \sup _{x \in X}\left|\left(Z^{-1} \partial_{n} Z\right)(x)\right|<\infty .
$$

By the chain rule, for any $R>1$,

$$
\partial_{n}|F(x)|_{B^{*}}^{R}=\left\{\begin{array}{cc}
R|F(x)|_{B^{*}}^{R-1}\left(\partial_{n} F(x), \partial|F(x)|_{B^{*}}\right)_{H}, & F(x) \neq 0 \\
0, & F(x)=0
\end{array}\right.
$$


and

$$
\partial_{n}|x|_{B}^{R}=\left\{\begin{array}{cc}
R|x|_{B^{*}}^{R-1}\left(\partial|x|_{B}, \varphi_{n}\right)_{H}, & x \neq 0 \\
0, & x=0
\end{array}\right.
$$

which can be readily estimated for all $x \in X$ as

$$
\begin{gathered}
\left.\left.\left|\partial_{n}\right| F(x)\right|_{B^{*}} ^{2 Q}|\leq 2 Q| F(x)\right|_{B^{*}} ^{2 Q-1}\left|\partial_{n} F(x)\right|_{B^{*}} \\
\left.\left.\left|\partial_{n}\right| x\right|_{B} ^{2 Q}|\leq 2 Q| x\right|_{B} ^{2 Q-1}\left|\varphi_{n}\right|_{B}
\end{gathered}
$$

Herewith, by $(\mathbf{F}),(6.5)$ and Young's inequality

$$
a^{R-1} b \leq \frac{R-1}{R} a^{R}+\frac{1}{R} b^{R}, \quad \forall a, b \in \mathbb{R}_{+},
$$

we have that uniformly for all $x \in X$,

$$
\begin{aligned}
\left|\left(Z^{-1} \partial_{n} Z\right)(x)\right| & \leq 2 Q \varepsilon \frac{|F(x)|_{B^{*}}^{2 Q-1}\left|\partial_{n} F(x)\right|_{B^{*}}+\kappa|x|_{B}^{2 Q-1}}{1+\varepsilon|F(x)|_{B^{*}}^{2 Q}+\varepsilon|x|_{B}^{2 Q}} \\
& \leq \mathcal{Z}:=2 Q\left[2 \mathcal{K}_{0}+\varepsilon^{\frac{1}{2 Q}}\left(\mathcal{L}_{0}+\kappa\right)\right] .
\end{aligned}
$$

Then, substituting (6.21) into (6.16) and using both $\left(\mathbf{L}_{1}\right)$ and $\left(\mathbf{L}_{2}\right)$, we conclude that

$$
\partial_{n} f_{n, \varepsilon}(x) \leq\left\{C_{Q, 1}[\tilde{L}(x)]^{Q}+C_{Q, 2}[\tilde{L}(x)]^{Q-1}\left|\left(F(x), \varphi_{n}\right)_{H}\right|\right\} Z^{-1}(x)
$$

with the constants

$$
C_{Q, 1}:=\mathcal{K}_{1}\left[1+(Q-1) \mathcal{K}_{2}\right], \quad C_{Q, 2}:=C_{Q, \varepsilon, 2}:=\mathcal{Z}+(Q-1) \mathcal{K}_{1} .
$$

On the other hand, by the (IbP)-formula (6.8),

$$
\begin{gathered}
\int_{X}[\tilde{L}(x)]^{Q-1}\left\{\lambda_{n}\left(F(x), \varphi_{n}\right)_{H}\left(x, \varphi_{n}\right)_{H}+\left(F(x), \varphi_{n}\right)_{H}^{2}\right\} Z^{-1}(x) d \mu(x) \\
=\int_{X} \partial_{n} f_{n, \varepsilon}(x) d \mu(x),
\end{gathered}
$$

and hence, combining (6.22) and (6.23),

$$
\begin{aligned}
\int_{X}[ & \tilde{L}(x)]^{Q-1}\left(F(x), \varphi_{n}\right)_{H}\left(x, \varphi_{n}\right)_{H} Z^{-1}(x) d \mu(x) \\
& \leq \lambda_{n}^{-1} \int_{X}[\tilde{L}(x)]^{Q-1}\left\{C_{Q, 1} \tilde{L}(x)-\left[\left(F(x), \varphi_{n}\right)_{H}^{2}-C_{Q, 2}\left|\left(F(x), \varphi_{n}\right)_{H}\right|\right]\right\} Z^{-1}(x) d \mu \\
& \leq \lambda_{n}^{-1} \int_{X}\left\{C_{Q, 1}[\tilde{L}(x)]^{Q}+\frac{1}{4} C_{Q, 2}^{2}[\tilde{L}(x)]^{Q-1}\right\} Z^{-1}(x) d \mu(x) .
\end{aligned}
$$

Now let us take the sum of the inequalities (6.24) over all $n \in \mathbb{N}$, keeping in mind that $\sum_{n=1}^{\infty} \lambda_{n}^{-1}=\operatorname{Tr}_{H} A^{-1}<\infty$ and that (due to (6.5)) uniformly for all $K \in \mathbb{N}$,

$$
\left|\sum_{n=1}^{K}\left(F(x), \varphi_{n}\right)_{H}\left(x, \varphi_{n}\right)_{H}\right|=\left|\left(F(x), \mathbb{P}_{K} x\right)_{H}\right| \leq \varsigma \mid\left(\left.F(x)\right|_{B^{*}}|x|_{B} .\right.
$$

Then Lebesgue's dominated convergence theorem yields that

$\left(1-C_{Q, 1} \operatorname{Tr}_{H} A^{-1}\right) \int_{X}[\tilde{L}(x)]^{Q} Z^{-1}(x) d \mu(x) \leq\left(\frac{1}{4} C_{Q, 2}^{2}+l+\varepsilon\right) \int_{X}[\tilde{L}(x)]^{Q-1} Z^{-1}(x) d \mu(x)$. 
But according to (6.11) we have that $\Xi_{Q-1}:=C_{Q, 1} \operatorname{Tr}_{H} A^{-1}<1$, and thus Hölder's inequality together with (6.23) and (6.25) implies that

$$
\begin{aligned}
(1- & \left.\Xi_{Q-1}\right)\left\{\int_{X}[\tilde{L}(x)]^{Q} Z^{-1}(x) d \mu(x)\right\}^{\frac{1}{Q}} \\
& \leq\left\{Q\left[2 \mathcal{K}_{0}+\varepsilon^{\frac{1}{2 Q}}\left(\mathcal{L}_{0}+\kappa\right)\right]+\frac{1}{2}(Q-1) \mathcal{K}_{1}\right\}^{2}+l+\varepsilon .
\end{aligned}
$$

Finally, letting $\varepsilon \searrow 0$ in (6.26), from Fatou's lemma we obtain that

$$
\left(1-\Xi_{Q-1}\right)\left\{\int_{X}\left[L_{H}^{F}(x)+l\right]^{Q} d \mu(x)\right\}^{\frac{1}{Q}} \leq\left[2 Q \mathcal{K}_{0}+\frac{1}{2}(Q-1) \mathcal{L}_{1}\right]^{2}+l,
$$

and thus

$$
\left\{\int_{X}\left|L_{H}^{F}(x)\right|^{Q} d \mu(x)\right\}^{\frac{1}{Q}} \leq\left\{\left[2 Q \mathcal{K}_{0}+\frac{1}{2}(Q-1) \mathcal{K}_{1}\right]^{2}+2 l\right\}\left(1-\Xi_{Q-1}\right)^{-1}<\infty
$$

which implies the required uniform integrability (6.12).

6.2.2. Integrability of partial logarithmic derivatives and consequences. Here we consider some direct applications of Theorem 6.2 proved above. First, let us analyze in more detail its assumptions. Note that the partial logarithmic derivatives (6.5) can be obviously decomposed with arbitrary $a \in \mathbb{R}$ as

$$
\begin{gathered}
b_{n}(x):=-\left(\tilde{A} \varphi_{n}, x\right)_{H}-\left(\tilde{F}(x), \varphi_{n}\right)_{H}, \quad n \in \mathbb{N}, x \in X, \\
\text { where } \tilde{A}:=A+a^{2} \mathbf{1} \text { and } \tilde{F}(x):=F(x)-a^{2} x .
\end{gathered}
$$

Since $\operatorname{Tr}_{H} A^{-1}<\infty$, one gets $\operatorname{Tr}_{H} \tilde{A}^{-1}<\varepsilon$ for any given $\varepsilon>0$ by choosing a large enough $a^{2} \geq a^{2}(\varepsilon)>0$. On the other hand, as can easily be seen from the definitions (6.2), (6.5) and (6.28), $\tilde{F}$ also satisfies Assumption $(\mathbf{F})$ but with the constants $\tilde{\mathcal{K}}_{0}:=\mathcal{K}_{0}\left(1+a^{2} \iota^{2}\right)$ and $\tilde{\mathcal{L}}_{0}:=\mathcal{L}_{0}+a^{2} \kappa$. Moreover, let us suppose (having in mind the single loop case with Assumption $\left(\mathbf{V}_{\mathbf{i i i}}\right)$ on the one-particle potentials $V_{k}$, see Remark 6.1) that $L_{H}^{F}$ satisfies additionally the following growth condition at infinity:

$\left(\mathbf{L}_{3}\right)$ there exist $R \geq 2, \mathcal{K}_{3}>0$ and $\mathcal{L}_{3} \geq 0: \quad|x|_{B}^{R} \leq \mathcal{K}_{3} L_{H}^{F}(x)+\mathcal{L}_{3} ;$

which in turn, when $R>2$, implies the standard coercivity property:

$\left(\mathbf{L}_{4}\right)$ for all $\mathcal{K}_{4}>0$ and $\mathcal{L}_{4} \geq 0: \quad|x|_{H}^{2} \leq \mathcal{K}_{4} L_{H}^{F}(x)+\mathcal{L}_{4}$.

From $\left(\mathbf{L}_{4}\right)$ obviously for any $\delta_{1}, \delta_{2}>0$ and $0<\mathcal{K}_{4} \leq \delta_{1}\left(a^{2}+\delta_{2}\right)^{-1}$,

$$
\begin{aligned}
L_{H}^{\tilde{F}}(x):=(\tilde{F}(x), x)_{H} & =\left(1-\delta_{1}\right) L_{H}^{F}(x)+\left(\delta_{1} L_{H}^{F}(x)-a^{2}|x|_{H}^{2}\right) \\
& \geq\left(1-\delta_{1}\right) L_{H}^{F}(x)+\delta_{2}|x|_{H}^{2}-\delta_{1} \mathcal{L}_{4} \mathcal{K}_{4}^{-1},
\end{aligned}
$$

so that $L_{H}^{\tilde{F}}$ also satisfies Assumptions $\left(\mathbf{L}_{1}\right)$ and $\left(\mathbf{L}_{2}\right)$ with corresponding constants

$$
\tilde{\mathcal{K}}_{1}:=\mathcal{K}_{1}\left(1-\delta_{1}\right)^{-1} \text { and } \tilde{\mathcal{K}}_{2}:=\mathcal{K}_{2}\left(1-\delta_{1}\right)^{-1} .
$$

Thus, for any given $Q \geq 1$, by a proper decomposition (4.28) with $a^{2} \geq a^{2}\left(Q, \mathcal{K}_{1}, \mathcal{K}_{2}\right)>0$ we can always achieve that

$$
\tilde{\Xi}_{Q-1}:=\tilde{\mathcal{K}}_{1}\left[1+(Q-1) \tilde{\mathcal{K}}_{2}\right] \operatorname{Tr}_{H} \tilde{A}^{-1}<1 .
$$

All together, this yields the following modification of Theorem 6.2:

Theorem 6.2' (Refinement of Theorem 6.2). Along with $(\mathbf{F}),\left(\mathbf{L}_{1}\right)$ and $\left(\mathbf{L}_{2}\right)$, let also Assumption $\left(\mathbf{L}_{4}\right)$ hold. Then the coercivity functional $L_{H}^{F}$ possesses the integrability property (4.12) for all $Q \geq 1$. 
The next statement is readily apparent from Theorem 6.2 and its proof.

Corollary 6.3 (Integrability of Polynomials and Partial Logarithmic Derivatives). Let the assumptions of Theorem 6.2 hold and additionally suppose that

$$
\Xi_{Q-1}<1 \text { for some fixed } Q \geq 1 \text {. }
$$

Then

$$
\begin{gathered}
\left.\sup _{\mu \in \mathcal{M}^{b}(X)} \sup _{n \in \mathbb{N}} \int_{X}\left|\left(x, \varphi_{n}\right)_{H}\right|^{Q} d \mu(x)\right\}=: \mathcal{C}_{Q}<\infty, \\
\sup _{\mu \in \mathcal{M}^{b}(X)} \int_{X}\left|b_{n}(x)\right|^{Q} d \mu(x)=: \mathcal{C}_{n, Q}<\infty, \quad \forall n \in \mathbb{N},
\end{gathered}
$$

and hence the (IbP)-formula (6.8) can be extended to all functions $f \in C_{b, l o c}^{1}(X)$ satisfying the extra growth condition for all $n \in \mathbb{N}$ :

$$
\text { there exists } C=C(f, n)>0:|f|+\left|\partial_{n} f\right| \leq C\left(1+L_{H}^{F}\right)^{Q-1} \text {. }
$$

Proof. The integrability of $b_{n}^{Q}(x)$ and $\left(x, \varphi_{n}\right)_{H}^{Q}$ uniformly w.r.t. all $\mu \in \mathcal{M}^{b}(X)$ is evident from $\left(\mathbf{L}_{1}\right)$ and (6.12). In order to extend the (IbP)-formula (6.8) to functions satisfying the growth condition (6.31), we construct a proper approximation of any such $f$ by $f_{\varepsilon} \in C_{\text {dec }}^{1}(X), 0<\varepsilon \leq 1$. Namely, define $f_{\varepsilon}(x)=f(x) Z_{\varepsilon}^{-1}(x)$, where $Z_{\varepsilon}$ is given by (6.15). Using the upper bound (6.20) for $\left|Z_{\varepsilon}^{-1} \partial_{n} Z_{\varepsilon}\right|$, it is easy to check that

$$
f_{\varepsilon} \rightarrow f, \partial_{n} f_{\varepsilon} \rightarrow \partial_{n} f, \varepsilon \searrow 0, \text { and }\left|f_{\varepsilon}\right|+\left|\partial_{n} f_{\varepsilon}\right| \leq C_{f, n}\left(1+L_{H}^{F}\right)^{Q-1} \text {. }
$$

Then the validity of (6.8) for this $f$ can be shown in a standard way by Lebesgue's dominated convergence theorem applied to both sides of the identity $\int_{X} \partial_{n} f_{\varepsilon} d \mu=-\int_{X} f_{\varepsilon} b_{n} d \mu$ when $\varepsilon \searrow 0$.

Remark 6.4. It would be worthwhile to compare our method with a usual Lyapunov function approach to the study of invariant distributions of stochastic differential equations (for its rigorous implementation in infinite-dimensional spaces we refer to [BR01]). Namely, keeping fixed the assumptions of Subsect. 6.1, let us consider the SDE

$$
d x_{t}=-\frac{1}{2}\left[A x_{t}+F\left(x_{t}\right)\right] d t+d w_{t}, \quad t>0,
$$

where $w_{t}, t \geq 0$, is a standard Wiener process with the identity correlation operator in $H$. Let a diffusion process $x_{t}, t \geq 0$, taking values in a Polish space $X \subset H$, be a solution to $(6.32)$ and let $(\mathbb{H}, \mathcal{D}(\mathbb{H}))$ be a generator of its associated Feller transition semigroup $\mathbb{P}_{t}, t \geq 0$, in $C_{b}(X)$. Then all invariant measures $\mu$ for $x_{t}, t \geq 0$, are also infinitesimally invariant, that is, $\int_{X} \mathbb{H} u d \mu=0, \forall u \in \mathcal{D}(\mathbb{H})$ (cf. Subsect. 5.3). For this reason in BR01 one deals directly with measures $\mu$ on $(X, \mathcal{B}(X))$ (including all those satisfying the (IbP)-formulas (6.8)) defined as solutions of the equation

$$
\int \mathbb{H} u d \mu=-\sum_{n \in \mathbb{N}} \int\left(\partial_{n}+b_{n}\right) \partial_{n} u d \mu=0
$$

on certain classes of test functions $u$ from $\mathcal{D}(\mathbb{H})$. If there exists a Lyapunov function $\phi: X \rightarrow \mathbb{R}$ such that $\mathbb{H} \phi \leq-g+c$, then one readily gets the uniform estimate $\int g d \mu \leq c$ for all distributions $\mu$ solving (6.33). The latter would mean, in the proof of Theorem 6.2 , a special choice of $\left(f_{n}\right)_{n \in \mathbb{N}}$ being of a gradient type, i.e., $f_{n}=\partial_{n} \phi, \forall n \in \mathbb{N}$. But this is not the case for the test functions we have used in the proof and thus our method is not directly applicable to invariant measures. To this end, we mention that for general nonlinear drifts $F: X \rightarrow X^{*}$ (except the case of $F$ having at most linear growth, cf. [BR01, Sect.7]) by now there are no analytic proofs (i.e., without using the SDE (6.34)) of the existence and a priori estimates for the invariant measures $\mu$ as solutions of (6.33). 
6.2.3. Finiteness of polynomial (exponential) moments. In this subsection we derive sufficient conditions for the integrability of $|x|_{B}^{Q}$ for all $Q \geq 1$ (and, moreover, $\exp \lambda|x|_{B}$ for all $\lambda \in \mathbb{R})$ w.r.t. $\mu \in \mathcal{M}^{b}(X)$.

Theorem 6.5 (A Priori Polynomial Moment Estimate). Let Assumptions $(\mathbf{F}),\left(\mathbf{L}_{1}\right)$ and $\left(\mathbf{L}_{2}\right)$ of the Theorem 6.2 hold and let us suppose that

$$
\Xi_{0}:=\mathcal{K}_{1} \operatorname{Tr}_{H} A^{-1}<1 .
$$

If, in addition, the asymptotic growth assumption on the coercivity functional

$\left(\mathbf{L}_{B}\right) \lim _{x \in X,|x|_{B} \rightarrow \infty} L_{H}^{F}(x)=+\infty$

holds, then the following moment estimate is satisfied for all $Q \geq 1$ :

$$
\sup _{\mu \in \mathcal{M}^{b}(X)} \int_{X}|x|_{B}^{Q} d \mu(x) \leq \mathcal{C}_{Q}^{\prime \prime}<\infty .
$$

Moreover, in this case the (IbP)-formula (6.8) can be extended to all $f \in C_{b, l o c}^{1}(X)$ satisfying the following polynomial growth condition for every fixed $n \in \mathbb{N}$ with corresponding $Q:=Q(f, n) \geq 1$ and $C:=C(f, n)>0$ :

$$
|f(x)|+\left|\partial_{n} f(x)\right| \leq C\left(1+|x|_{B}\right)^{Q-1}, x \in X .
$$

Proof. First we note that $\Xi_{0}<1$ implies by Theorem 6.2 ,

$$
\sup _{\mu \in \mathcal{M}^{b}(X)} \int_{X}\left|L_{H}^{F}(x)\right| d \mu(x) \leq \mathcal{C}_{1}^{\prime}<\infty .
$$

Below we will also use the notation and the relevant estimates (6.17)-(6.27) from the proof of Theorem 6.2.

So, fix $Q>2$ and $n \in \mathbb{N}$, and consider the following family of test functions $g \in$ $C_{d e c}^{1}(X), 0<\varepsilon \leq 1$,

$$
g(x):=g_{n, \varepsilon}(x):=|x|_{B}^{Q-1}\left(F(x), \varphi_{n}\right)_{H} Z^{-1}(x), \quad x \in X,
$$

with the cut-off term $Z(x):=Z_{\varepsilon}(x)$ given by (6.15). Their partial derivatives $\partial_{n} g$ in the direction $\varphi_{n}$ can be estimated for all $x \in X$ as follows:

$$
\begin{aligned}
\partial_{n} g_{n, \varepsilon}(x) \leq|x|_{B}^{Q-2}\left\{|x|_{B}\left(\partial_{n} F(x), \varphi_{n}\right)_{H}+(Q-1)\left|\left(F(x), \varphi_{n}\right)_{H}\right| \cdot\left|\varphi_{n}\right|_{B}\right\} Z^{-1} \\
+\mathcal{Z}|x|_{B}^{Q-1}\left|\left(F(x), \varphi_{n}\right)_{H}\right| Z^{-1} .
\end{aligned}
$$

Thus, by $\left(\mathbf{L}_{1}\right)$ and the (IbP)-formula (6.8),

$$
\begin{aligned}
& \int_{X}|x|_{B}^{Q-1}\left(F(x), \varphi_{n}\right)_{H}\left(x, \varphi_{n}\right)_{H} Z^{-1}(x) d \mu(x) \\
& \leq \lambda_{n}^{-1} \int_{X}\left\{|x|_{B}^{Q-2}\left[|x|_{B}+(Q-1) \kappa\right]\left[\mathcal{K}_{1} L_{H}^{F}(x)+\mathcal{L}_{1}\right]\right. \\
&\left.\quad-|x|_{B}^{Q-1}\left[\left(F(x), \varphi_{n}\right)_{H}^{2}-\mathcal{Z}\left|\left(F(x), \varphi_{n}\right)_{H}\right|\right]\right\} Z^{-1}(x) d \mu(x)
\end{aligned}
$$

and herewith, summing over all $n \in \mathbb{N}$, we obtain

$$
\begin{aligned}
(1- & \left.\mathcal{K}_{1} \operatorname{Tr}_{H} A^{-1}\right) \int_{X}|x|_{B}^{Q-1} L_{H}^{F}(x) Z^{-1}(x) d \mu(x) \\
\leq & \operatorname{Tr}_{H} A^{-1}\left(\mathcal{L}_{1}+\frac{1}{4} \mathcal{Z}^{2}\right) \int_{X}|x|_{B}^{Q-1} Z^{-1}(x) d \mu(x) \\
& +\operatorname{Tr}_{H} A^{-1}(Q-1) \kappa \int_{X}|x|_{B}^{Q-2}\left[\mathcal{K}_{1} L_{H}^{F}(x)+\mathcal{L}_{1}\right] Z^{-1}(x) d \mu(x) .
\end{aligned}
$$


Then an elementary application of Young's inequality in the last line of (6.38) gives that for any fixed but small enough $\delta>0$ (more precisely, such that $\delta<\left(\operatorname{Tr}_{H} A^{-1}\right)^{-1}-\mathcal{K}_{1}$ ),

$$
\begin{aligned}
& \int_{X}|x|_{B}^{Q-1} L_{H}^{F}(x) Z^{-1}(x) d \mu(x) \\
& \quad \leq C_{Q, 1} \int_{X}|x|_{B}^{Q-1} Z^{-1}(x) d \mu(x)+C_{Q, 2} \int_{X}\left(\left|L_{H}^{F}(x)\right|+1\right) d \mu(x)
\end{aligned}
$$

with positive constants

$$
C_{Q, 1}:=\frac{\left(\mathcal{L}_{1}+\frac{1}{4} \mathcal{Z}^{2}+\delta\right) \operatorname{Tr}_{H} A^{-1}}{\left(1-\left(\mathcal{K}_{1}+\delta\right) \operatorname{Tr}_{H} A^{-1}\right)} \quad \text { and } \quad C_{Q, 2}:=C_{Q, 2}\left(\mathcal{K}_{1}, \mathcal{L}_{1}\right)
$$

Taking into account (6.36) and choosing any $\rho>0$ from Assumption $\left(\mathbf{L}_{B}\right)$ large enough so that

and hence obviously

$$
L_{H}^{F}(x) \geq C_{Q, 1}+1 \quad \text { when } \quad|x|_{B} \geq \rho,
$$

$$
|x|_{B}^{Q-1} L_{H}^{F}(x) \geq\left(C_{Q, 1}+1\right)|x|_{B}^{Q-1}-\left(C_{Q, 1}+1-l\right) \rho^{Q-1}, \quad \forall x \in X,
$$

we get that

$$
\sup _{0<\varepsilon \leq 1} \int_{X}|x|_{B}^{Q-1} Z_{\varepsilon}^{-1}(x) d \mu(x) \leq\left(C_{Q, 1}+1-l\right) \rho^{Q-1}+C_{Q, 2}\left(\mathcal{C}_{1}^{\prime}+1\right)<\infty .
$$

Finally, letting $\varepsilon \searrow 0$ in (6.41), from Fatou's lemma we obtain the required estimate (6.34).

Corollary 6.3' (Refinement of Corollary 6.3). Suppose that the partial logarithmic derivatives $b_{n}$ (or, even more, the coercivity functional $L_{H}^{F}$ ), have at most polynomial growth, i.e.,

$\left(\mathbf{P}_{B}\right)$ there exist $\mathcal{K}_{n}^{\prime}, \mathcal{L}_{n}^{\prime}, R_{n}^{\prime}>0$ (resp. $\left.\mathcal{K}^{\prime}, \mathcal{L}^{\prime}, R^{\prime}>0\right)$ such that for all $x \in X$,

$$
\left|b_{n}(x)\right| \leq \mathcal{K}_{n}^{\prime}|x|_{B}^{R_{n}^{\prime}}+\mathcal{L}_{n}^{\prime} \quad\left(\text { resp. }\left|L_{H}^{F}(x)\right| \leq \mathcal{L}^{\prime}|x|_{B}^{R^{\prime}}+\mathcal{L}^{\prime}\right) .
$$

Then under the assumptions of Theorem 6.5 for all $Q \geq 1$,

$$
\sup _{\mu \in \mathcal{M}^{b}(X)} \int_{X}\left|b_{n}(x)\right|^{Q} d \mu(x)<\infty \quad\left(\text { resp. } \sup _{\mu \in \mathcal{M}^{b}(X)} \int_{X}\left|L_{H}^{F}(x)\right|^{Q} d \mu(x)<\infty\right) .
$$

Suppose that the coercivity functional $L_{H}^{F}$ satisfies also Assumption $\left(\mathbf{L}_{3}\right)$ with some fixed $R>2$ and $\mathcal{K}_{3}, \mathcal{L}_{3}>0$, and thus surely $\left(\mathbf{L}_{B}\right)$. Let us use again the decomposition (6.28) of the logarithmic derivative $b$ with $a^{2}>0$ large enough. Then, as is readily seen from (6.29), $\left(\mathbf{L}_{1}\right)$ and $\left(\mathbf{L}_{B}\right)$ respectively imply

$$
\tilde{\Xi}_{0}:=\tilde{\mathcal{K}}_{1} \operatorname{Tr}_{H} \tilde{A}^{-1}<1 \text { and } \lim _{|x|_{B} \rightarrow \infty} L_{H}^{\tilde{F}}(x)=\infty .
$$

Thus, we get the following modification of Theorem 6.5:

Theorem 6.5' (Refinement of Theorem 6.5). Along with $(\mathbf{F}),\left(\mathbf{L}_{1}\right)\left(\mathbf{L}_{2}\right)$, let Assumption $\left(\mathbf{L}_{3}\right)$ hold with some fixed $R>2$. Then the moment estimate (6.36) is satisfied for all $Q \geq 1$.

Remark 6.6. (i) In order to get that

$$
\sup _{\mu \in \mathcal{M}^{b}(X)} \int_{X}|x|_{H}^{Q} d \mu(x)<\infty, \quad \text { for all } Q \geq 1
$$

it suffices to just replace Assumption $\left(\mathbf{L}_{B}\right)$ in the formulation of Theorem 6.5 by a weaker one, namely:

$$
\left(\mathbf{L}_{H}\right) \lim _{x \in X,|x|_{H} \rightarrow \infty} L_{H}^{F}(x)=\infty .
$$


(ii) In fact, under the assumptions of Theorem 6.5, an a priori exponential moment estimate holds:

$$
\sup _{\mu \in \mathcal{M}^{b}(X)} \int_{X} \exp \lambda|x|_{B} d \mu(x) \leq \mathcal{C}_{\lambda}^{\prime \prime}<\infty, \quad \text { for all } \lambda \in \mathbb{R} .
$$

The proof of estimate (6.43) is similar to that of (6.34), but with the family of test functions $g \in C_{b}^{1}(X \backslash\{0\})$, indexed by $n \in \mathbb{N}$ and $0<\varepsilon \leq 1$,

$$
g(x):=g_{n, \varepsilon}(x):=\frac{\left(F(x), \varphi_{n}\right)_{H} \exp \lambda|x|_{B}}{1+\varepsilon|F(x)|_{B^{*}}^{2}+\varepsilon \exp 2 \lambda|x|_{B}}, \quad x \in X .
$$

6.2.4. Support properties of symmetrizing measures. Below we study a relation between the support properties of measures $\mu \in \mathcal{M}^{b}(X)$ and spectral properties of the operator $A$ in $H$. For this purpose we introduce the scale of Hilbert spaces defined in terms of the powers of $A$ :

$$
H^{\alpha}:=\left\{\left.x \in H|| x\right|_{H^{\alpha}}:=\left[\sum_{n \in \mathbb{N}} \lambda_{n}^{\alpha}\left(x, \varphi_{n}\right)_{H}^{2}\right]^{1 / 2}<\infty\right\}, \alpha \geq 0 ; \quad H^{0}:=H .
$$

Theorem 6.7 (A Priori Moment Estimates for Sobolev Norms). Let the assumptions of Theorem 6.2 hold and suppose that

$$
\Xi_{2 Q-1}<1 \text { for some } Q \geq 1 \text {. }
$$

If, in addition, the following assumption on the linear part $A$ of the logarithmic derivative holds:

$\left(\mathbf{T}_{\alpha}\right) \operatorname{Tr}_{H} A^{\alpha-1}<\infty$ for some $\alpha \geq 0$, then $\mu\left(X \cap H^{\alpha}\right)=1$ and, moreover,

$$
\sup _{\mu \in \mathcal{M}^{b}(X)} \int_{X}|x|_{H^{\alpha}}^{Q+1} d \mu \leq \mathcal{C}_{Q, \alpha}<\infty .
$$

Proof. Let for instance $Q>2$ and let us apply the (IbP)-formula (6.8) along basis vectors $\varphi_{n}, n \in \mathbb{N}$, to the following cylinder test functions $g \in \mathcal{F} C^{1}(X)$ indexed by $K, n \in \mathbb{N}$, $K \geq n$,

$$
g(x):=g_{K, n}(x):=\left(x, \varphi_{n}\right)_{H}\left|P_{K} x\right|_{H^{\alpha}}^{Q-1}, \quad x \in X
$$

(which is allowed in this case by Corollary 6.3 ; if $Q \leq 2$ one obviously takes $\partial_{n}^{ \pm} g_{n}$ instead of $\left.\partial_{n} g_{n}\right)$. First, by virtue of (6.18),

$$
\partial_{n} g_{K, n}(x)=\left\{\begin{array}{cc}
\left|P_{K} x\right|_{H^{\alpha}}^{Q-1}+(Q-1)\left|P_{K} x\right|_{H^{\alpha}}^{Q-3}\left(P_{K} x, \varphi_{n}\right)_{H}\left(P_{K} x, \varphi_{n}\right)_{H^{\alpha}}, & P_{K} x \neq 0 \\
0, & P_{K} x=0,
\end{array}\right.
$$

and thus

$$
\left|\partial_{n} g_{K, n}(x)\right| \leq Q\left|P_{K} x\right|_{H^{\alpha}}^{Q-1}, \quad \forall x \in X .
$$

Hence the (IbP)-formula yields for $1 \leq n \leq K$,

$$
\int_{X}\left[\lambda_{n}\left(x, \varphi_{n}\right)_{H}^{2}+\left(F(x), \varphi_{n}\right)_{H}\left(x, \varphi_{n}\right)_{H}\right]\left|P_{K} x\right|_{H^{\alpha}}^{Q-1} d \mu(x) \leq Q \int_{X}\left|P_{K} x\right|_{H^{\alpha}}^{Q-1} d \mu(x),
$$

and herewith by Young's inequality,

$$
\begin{aligned}
& \lambda_{n} \int_{X}\left(x, \varphi_{n}\right)^{2}\left|P_{K} x\right|_{H^{\alpha}}^{Q-1} d \mu(x) \\
& \quad \leq \int_{X}\left[\left|P_{K} x\right|_{H^{\alpha}}^{Q}+Q\left|P_{K} x\right|_{H^{\alpha}}^{Q-1}\right] d \mu(x)+\sup _{n \in \mathbb{N}} \int_{X}\left|\left(F(x), \varphi_{n}\right)\left(x, \varphi_{n}\right)\right|^{Q} d \mu(x) .
\end{aligned}
$$


Summing over $1 \leq n \leq K$ and using $\left(\mathbf{T}_{\alpha}\right),(6.12)$ and (6.46), we conclude from (6.50) that for all $K \in \mathbb{N}$ and $0<\delta \leq 1$,

$$
\begin{aligned}
& \int_{X}\left|P_{K} x\right|_{H^{\alpha}}^{Q+1} d \mu(x) \\
& \leq \operatorname{Tr}_{H} A^{\alpha-1}\left\{\delta \int_{X}\left|P_{K} x\right|_{H^{\alpha}}^{Q+1} d \mu(x)+\int_{X}\left(\mathcal{K}_{1} L_{H}^{F}(x)+\mathcal{L}_{1}\right)^{2 Q} d \mu(x)+C(Q, \delta)\right\} \\
& \leq \operatorname{Tr}_{H} A^{\alpha-1}\left\{\delta \int_{X}\left|P_{K} x\right|_{H^{\alpha}}^{Q+1} d \mu(x)+C_{Q}\left(\mathcal{K}_{1}, \mathcal{L}_{1}, \mathcal{C}_{2 Q}^{\prime}, \delta\right)\right\} .
\end{aligned}
$$

Finally, choosing any $0<\delta<\left(\operatorname{Tr}_{H} A^{\alpha-1}\right)^{-1}$ and then letting $K \rightarrow \infty$ in (6.51), from Fatou's lemma we obtain the required estimate (6.47).

Using again the decomposition (6.28) of the logarithmic derivative $b$, we get the corresponding modification of Theorem 6.7 :

Theorem 6.7' (Refinement of Theorem 6.7). Along with $(\mathbf{F}),\left(\mathbf{L}_{1}\right)$ and $\left(\mathbf{L}_{2}\right)$, let Assumptions $\left(\mathbf{L}_{4}\right)$ and $\left(\mathbf{T}_{\alpha}\right)$ hold. Then for all $Q \geq 1$ the uniform integrability estimate (5.49) for the Sobolev norms $|\cdot|_{H^{\alpha}}$ holds.

\section{Symmetrizing measures on Banach (e.g. loop) lattices}

Having in mind applications to the Euclidean Gibbs measures on the "loop lattice" $\Omega:=\left[C_{\beta}\right]^{\mathbb{Z}^{d}}$, we further enrich the abstract setting of the previous section by adding a lattice structure. So, our aim here will be to develop an abstract framework for symmetrizing measures $\mu \in \mathcal{M}^{b}(\mathcal{X})$ on Banach lattices $\mathcal{X}:=X^{\mathbb{Z}^{d}}$. Such measures will be defined in Subsect. 7.1 as the solutions to the (IbP)-formula (7.16). The required a priori estimates on $\mu \in \mathcal{M}^{b}(\mathcal{X})$ will be formulated in Subsect.7.2 as Theorems 7.1 and 7.3. In Subsect. 7.3 we come back to the Euclidean Gibbs measures and lastly, on the basis of the abstract results obtained, verify the validity of Hypotheses $(\mathrm{H})$ and $\left(\mathrm{H}_{l o c}\right)$ for them. Recall that these hypotheses were crucial for the proof in Sect. 5 of our Main Theorems I-III describing the properties of $\mu \in \mathcal{G}_{t}$. Let us also emphasize that here we do not touch at all such problems as existence and (what is an especially difficult and completely open problem) uniqueness for $\mu \in \mathcal{M}^{b}(\mathcal{X})$, since in applications to the Euclidean Gibbs measures we prefer more standard methods for their investigation (cf. Subsect. 2.6.1).

\section{1. (IbP)-formula on Banach lattices.}

7.1.1. Support spaces for symmetrizing measures. In order to include the case of Gibbs measures on loop lattices (for their (IbP)-description see Subsect.4.4), one can modify the abstract setting of Subsect. 6.1 as follows:

As before, let

$$
X \subset B \subset H \subset B^{*}
$$

be a rigging of the Hilbert space $\left(H,(\cdot, \cdot)_{H}\right)$ by a locally convex space $X$ and by a reflexive Banach space $\left(B,|\cdot|_{B}\right)$ and its dual $\left(B^{*},|\cdot|_{B^{*}}\right)$ with the properties (6.1)-(6.4). We only recall that in $H$ we fix an orthonormal basis $\operatorname{bas}(H)=\left\{\varphi_{n}\right\}_{n \in \mathbb{N}}$, indexed by any countable set, $\mathbb{N}$ say, and consisting of the eigenvectors $\varphi_{n}$ of the selfadjoint operator $A>0$, i.e., $A \varphi_{n}=\lambda_{n} \varphi_{n}$, with $\operatorname{Tr}_{H} A^{-1}=\sum_{n \in \mathbb{N}} \lambda_{n}^{-1}<\infty$.

On the other hand, let us be given one more rigging

$$
E_{+} \subset E_{0} \subset E_{-}
$$


of the Hilbert space $\left(E_{0},(\cdot, \cdot)_{0}\right)$ by Hilbert spaces $\left(E_{+},(\cdot, \cdot)_{+}\right)$and its dual $\left(E_{-},(\cdot, \cdot)_{-}\right)$. Again, as in (7.1), all the spaces in (7.2) are separable and all the embeddings are dense and continuous. Without loss of generality, let

$$
|\cdot|_{-} \leq|\cdot|_{0} \leq|\cdot|_{+} .
$$

What is important, the operators $O^{-}: E_{0} \rightarrow E_{-}$and $O^{+}: E_{+} \rightarrow E_{0}$ are supposed to have finite Hilbert-Schmidt norm

$$
\left\|O^{ \pm}\right\|_{H S}:=\left\|O^{-}: E_{0} \rightarrow E_{-}\right\|_{H S}=\left\|O^{+}:=E_{+} \rightarrow E_{0}\right\|_{H S}<\infty .
$$

The duality between $E_{+}$and $E_{-}$is given by the scalar product $(\cdot, \cdot)_{0}$ in $E_{0}$, and by the Riesz representation theorem there is a canonical isometry $\mathbf{I}: E_{-} \rightarrow E_{+}$defined by

$$
\left(e, e^{\prime}\right)_{0}=\left(e^{\prime}, e\right)_{0}=\left(\mathbf{I} e, e^{\prime}\right)_{+}=\left(e, \mathbf{I}^{-1} e^{\prime}\right)_{-}, \quad e \in E_{-}, e^{\prime} \in E_{+} .
$$

Let $\left\{e_{k}\right\}_{k \in \mathbb{Z}},\left\{e_{k}^{+}\right\}_{k \in \mathbb{Z}}$ and $\left\{e_{k}^{-}\right\}_{k \in \mathbb{Z}}$ be orthonormal bases in $E_{0}, E_{+}$and $E_{-}$, respectively, indexed by any countable set, with abelian group structure, say $\mathbb{Z}$. Due to (7.3) and (7.4), they can always be taken so that

$$
\begin{gathered}
e_{k} \in E_{+} \text {and } e_{k}^{+}=\gamma_{k}^{-1 / 2} e_{k}, e_{k}^{-}=\gamma_{k}^{1 / 2} e_{k} \\
\gamma_{k} \geq 1, \quad \sum_{k \in \mathbb{Z}} \gamma_{k}^{-1}=\left\|O^{ \pm}\right\|_{H S}^{2} \leq 1
\end{gathered}
$$

with some weight sequence $\gamma=\left\{\gamma_{k}\right\}_{k \in \mathbb{Z}}$. The latter means that one has the natural isomorphism $e \leftrightarrow\left\{\left(e, e_{k}\right)_{0}\right\}_{k \in \mathbb{N}}$ between the spaces $E_{0}, E_{+}, E_{-}$and the coordinate spaces $l^{2}(\mathbb{Z}), l^{2}(\mathbb{Z} ; \gamma), l^{2}\left(\mathbb{Z} ; \gamma^{-1}\right)$ (cf. Subsect. 3.2.2) respectively. To make things technically easier, we impose one more specific assumption on the rigging (7.2)

$$
\gamma_{k-j}=\gamma_{j-k} \leq \gamma_{k} \gamma_{j} \quad \forall k, j \in \mathbb{Z},
$$

which in particular implies that the shift operator

$$
T_{j}\left\{e_{k}\right\}_{k \in \mathbb{Z}}:=\left\{e_{k+j}\right\}_{k \in \mathbb{Z}}, \quad\left\|T_{j}\right\|_{l^{2}}=1, \quad j \in \mathbb{Z},
$$

is also bounded in all $l^{q}\left(\mathbb{Z} ; \gamma^{-q}\right), q \geq 1$, and $\left\|T_{j}\right\|_{l^{q}\left(\gamma^{-q}\right)} \leq \gamma_{j}$. This obviously yields the inclusion

$$
l^{2}\left(\mathbb{Z} ; \gamma^{-1}\right) \subset l^{1}\left(\mathbb{Z} ; \gamma^{-1}\right) \subseteq l^{q}\left(\mathbb{Z} ;\left\{\gamma_{k-j}^{-q}\right\}_{k \in \mathbb{N}}\right), \quad q \geq 1, j \in \mathbb{Z},
$$

with the following relations between the corresponding norms for arbitrary vectors $e \in$ $l^{2}\left(\mathbb{Z} ; \gamma^{-1}\right)$ :

$$
\gamma_{j}^{-1}|e|_{l^{q}\left(\left\{\gamma_{k-j}^{-q}\right\}_{k \in \mathbb{N}}\right)} \leq|e|_{l^{q}\left(\gamma^{-q}\right)} \leq|e|_{l^{1}\left(\gamma^{-1}\right)} \leq|e|_{l^{2}\left(\gamma^{-1}\right)}|| O^{ \pm} \|_{H S} \leq|e|_{l^{2}\left(\gamma^{-1}\right)} .
$$

We stress once more that the riggings (7.1), resp. (7.2), include the cases of single loop spaces, resp. spaces of scalar sequences over $\mathbb{Z}^{d}$, in Subsect. 3.2.1, resp. Subsect. 3.2.2, as special cases.

Thereafter, we define (having in mind, in particular, the spaces of loop sequences from Subsect. 3.2.3):

- the tangent Hilbert space $\left(\mathcal{H},\langle\cdot, \cdot\rangle_{\mathcal{H}}\right)$,

$$
\begin{aligned}
\mathcal{H} & :=l^{2}(\mathbb{Z} \rightarrow H) \\
& :=\left\{x=\left(x_{k}\right)_{k \in \mathbb{Z}} \in H^{\mathbb{Z}} \mid\|x\|_{\mathcal{H}}:=\langle x, x\rangle_{\mathcal{H}}^{1 / 2}:=\left[\sum_{k \in \mathbb{Z}}\left|x_{k}\right|_{H}^{2}\right]^{1 / 2}<\infty\right\} \\
& \cong E_{0} \otimes H:=\left\{x:=\sum_{k \in \mathbb{Z}} e_{k} \otimes x_{k} \mid\|x\|_{E_{0} \otimes H}=:\|x\|_{0}<\infty\right\}
\end{aligned}
$$


- the reflexive Banach space with smooth norm $\left(\mathcal{B},\|\cdot\|_{\mathcal{B}}\right)$,

$$
\begin{aligned}
\mathcal{B} & :=l^{2}\left(\mathbb{Z} \rightarrow B ; \gamma^{-1}\right) \\
& :=\left\{x=\left(x_{k}\right)_{k \in \mathbb{Z}} \in B^{\mathbb{Z}} \mid\|x\|_{\mathcal{B}}:=\left[\sum_{k \in \mathbb{Z}} \gamma_{k}^{-1}\left|x_{k}\right|_{B}^{2}\right]^{1 / 2}<\infty\right\} \\
& \cong E_{-} \otimes B:=\left\{x:=\sum_{k \in \mathbb{Z}} e_{k} \otimes x_{k} \mid\|x\|_{E_{-} \otimes B}=:\|x\|_{\mathcal{B}}<\infty\right\} ;
\end{aligned}
$$

- the locally convex Polish space $\mathcal{X}:=\mathcal{B} \cap X^{\mathbb{Z}}$, as a support space for measures to be considered, with the metric

$$
\rho_{\mathcal{X}}\left(x, x^{\prime}\right):=\left[\sum_{k \in \mathbb{Z}} \gamma_{k}^{-1}\left(\left|x_{k}-x_{k}^{\prime}\right|_{B}^{2}+\frac{\left|x_{k}-x_{k}^{\prime}\right|_{X}^{2}}{1+\left|x_{k}-x_{k}^{\prime}\right|_{X}^{2}}\right)\right]^{1 / 2} .
$$

7.1.2. Smooth functions and measures. Again, as in Subsects. 4.2.1 and 6.1, we define the spaces $C_{b}^{1}(\mathcal{X} ; h)$, resp. $C_{b, l o c}^{1}(\mathcal{X} ; h)$, of all functions $f: \mathcal{X} \rightarrow \mathbb{R}$ which are continuous and globally, resp. locally, bounded together with their partial derivative $\partial_{h} f: \mathcal{X} \rightarrow \mathbb{R}$ along a given direction $h \in \mathcal{X}$. Analogously,

$$
C_{b}^{1}(\mathcal{X}):=\bigcap_{h \in \mathcal{X}} C_{b}^{1}(\mathcal{X} ; h), \quad \text { resp. } \quad C_{b, l o c}^{1}(\mathcal{X}):=\bigcap_{h \in \mathcal{X}} C_{b, l o c}^{1}(\mathcal{X} ; h) .
$$

We fix an orthonormal basis bas $(\mathcal{H}):=\left\{h_{i}\right\}_{i \in \mathbb{Z}^{d+1}}$ in $\mathcal{H}$,

$$
h_{i}:=\left\{\delta_{k, j} \varphi_{n}\right\}_{j \in \mathbb{N}} \cong e_{k} \otimes \varphi_{n}, \quad i=(k, n) \in \mathbb{Z} \times \mathbb{N} .
$$

The corresponding subspaces of cylinder functions will be denoted by $\mathcal{F} C_{b}^{1}(\mathcal{X})$, resp. $\mathcal{F} C_{b, l o c}^{1}(\mathcal{X})$. For brevity, $\partial_{i} f:=\partial_{(k, n)} f$ will denote the derivative along the basis vector $h_{i}=h_{(k, n)}$.

Given any $k \in \mathbb{Z}$, let

$$
F_{k}^{0}: X \rightarrow B^{*} \text { and } G_{k}: \mathcal{X}_{-} \rightarrow B^{*}
$$

be some nonlinear mappings which are continuous and locally bounded together with their partial derivatives in all directions $\varphi_{n} \in \operatorname{bas}(H)$, resp. $e_{k} \otimes \varphi_{n} \in \operatorname{bas}(\mathcal{H}), n \in \mathbb{N}$. With regard to (4.38), (4.39) and (6.6), we define a measurable vector field $\mathcal{X} \ni x \rightarrow b(x):=$ $\left\{b_{i}(x)\right\}_{i \in \mathbb{Z} \times \mathbb{N}} \in \mathbb{R}^{\mathbb{Z} \times \mathbb{N}}$ (the so-called logarithmic gradient) by

$$
\begin{gathered}
b_{i}(x)=b_{(k, n)}(x):=-\left(A \varphi_{n}, x_{k}\right)_{H}-\left(F_{k}(x), \varphi_{n}\right)_{H}, \\
F_{k}(x):=F_{k}^{0}\left(x_{k}\right)+G_{k}(x), \quad i=(k, n) \in \mathbb{Z} \times \mathbb{N} .
\end{gathered}
$$

Here, along with the operator $A$ as the linear component of $b$, it is convenient to separate its nonlinear diagonal components $F_{k}^{0}\left(x_{k}\right)$ and resp. nondiagonal ones $G_{k}(x)$. From assumptions (6.3)-(6.5) on the eigenvectors $\varphi_{n}, n \in \mathbb{N}$, of the operator $A$ it follows that

$$
b_{(k, n)} \in C_{b, l o c}^{1}(\mathcal{X}) \quad \text { and } \quad\left|b_{(k, n)}(x)\right| \leq \kappa\left(\lambda_{n}\left|x_{k}\right|_{B^{*}}+\left|F_{k}(x)\right|_{B^{*}}\right) .
$$

Let $\mathcal{M}^{b}(\mathcal{X})$ denote the family of all Borel probability measures $\mu$ on $\mathcal{X}$, which satisfy for any $i=(k, n) \in \mathbb{Z} \times \mathbb{N}$ the $(I b P)$-formula

$$
\int_{\mathcal{X}} \partial_{(k, n)} f(x) d \mu(x)=-\int_{\mathcal{X}} f(x) b_{(k, n)}(x) d \mu(x)
$$

on the corresponding dense subset $C_{d e c}^{1}\left(\mathcal{X} ; h_{i}\right) \subset C_{b}^{1}\left(\mathcal{X} ; h_{i}\right)$ of all functions $f$ possessing the additional decay property

$$
\sup _{x \in \mathcal{X}}\left\{|f(x)|\left(1+\left|x_{k}\right|_{B^{*}}+\left|F_{k}(x)\right|_{B^{*}}\right)\right\}<\infty .
$$


7.2. A priori integrability properties. We start with a priori integrability properties of $\mu \in \mathcal{M}^{b}(\mathcal{X})$ supposing that such for sure exist, i.e., $\mathcal{M}^{b}(\mathcal{X}) \neq \emptyset$.

7.2.1. Main theorem. Theorem 7.1 presented below is an extension both of Theorems 6.2 and 6.5 to the case of measures on Banach (e.g. loop) lattices. Having in mind concrete applications to quantum lattice systems such as (3.1), from the very beginning we impose here the polynomial boundedness of the nonlinear nondiagonal terms $G_{k}(x)$ and strong enough coercivity properties of the nonlinear diagonal terms $F_{k}^{0}\left(x_{k}\right)$ in the presentation $(7.14)$ for the logarithmic derivatives $b_{(k, n)}$ (see Assumptions $\left(\mathbb{G}_{1}\right),\left(\mathbb{G}_{2}\right)$ and resp. $\left(\mathbb{L}_{3}\right)$ below).

According to Definition 6.1, we introduce the coercivity functionals corresponding to the vector fields $F_{k}^{0}: X \rightarrow B^{*}, k \in \mathbb{Z}$, w.r.t. the tangent Hilbert space $\mathcal{H}$ by

$$
L_{k}:=L_{H}^{F_{k}^{0}}: X \rightarrow \mathbb{R}, \quad L_{H}^{k}\left(x_{k}\right):=\left(F_{k}^{0}\left(x_{k}\right), x_{k}\right)_{H}, \quad x_{k} \in X .
$$

Theorem 7.1 (A Priori Moment Estimates and Integrability of Coercivity Functionals).

Fix some $R \geq 1$ and a weight system $\gamma=\left\{\gamma_{k}\right\}_{k \in \mathbb{Z}}$ with the properties (7.5)-(7.9). Let $\mathcal{J}=\left\{\mathcal{J}_{k, j}\right\}_{k, j \in \mathbb{N}}$ be an infinite symmetric matrix with nonnegative entries $\mathcal{J}_{k, j}=\mathcal{J}_{j, k} \geq$ 0 such that

(J) || $\mathcal{J} \mid \|:=\sup _{k \in \mathbb{Z}} \sum_{j \in \mathbb{Z}} \mathcal{J}_{k, j} \gamma_{k-j}^{R}<\infty$.

Furthermore, let the following assumptions on the vector fields $F_{k}^{0}: X \rightarrow B^{*}$ and $G_{k}$ : $\mathcal{X}_{-} \rightarrow B^{*}$ hold uniformly for all $x \in \mathcal{X}$ and $i=(k, n) \in \mathbb{Z} \times \mathbb{N}$ :

$\left(\mathbb{F}_{0}\right) \exists \mathcal{K}_{0}>0$ and $\mathcal{L}_{0} \geq 0: \quad\left|\partial_{n} F_{k}^{0}\left(x_{k}\right)\right|_{B^{*}} \leq \mathcal{K}_{0}\left(\left|F_{k}^{0}\left(x_{k}\right)\right|_{B^{*}}+\left|x_{k}\right|_{B}^{R}\right)+\mathcal{L}_{0} ;$

$\left(\mathbb{G}_{1}\right) \exists \mathcal{M}_{1}>0$ and $\mathcal{N}_{1} \geq 0$ : $\left|\left(G_{k}(x), \varphi_{n}\right)_{H}\right|+\left|\left(\partial_{i} G_{k}(x), \varphi_{n}\right)_{H}\right| \leq \mathcal{M}_{1} \sum_{j \in \mathbb{Z}} \mathcal{J}_{k, j}\left|x_{j}\right|_{B}^{R}+\mathcal{N}_{1} ;$

$\left(\mathbb{G}_{2}\right) \exists \mathcal{M}_{2}>0$ and $\mathcal{N}_{2} \geq 0:$

$$
\left|G_{k}(x)\right|_{B^{*}}+\left|\left(G_{k}(x), x_{k}\right)_{H}\right|+\left|\left(\partial_{i} G_{k}(x), x_{k}\right)_{H}\right| \leq \mathcal{M}_{2} \sum_{j \in \mathbb{Z}} \mathcal{J}_{k, j}\left|x_{j}\right|_{B}^{R}+\mathcal{N}_{2} ;
$$

and respectively for the coercivity functionals $L_{k}: X \rightarrow \mathbb{R}:$

$\left(\mathbb{L}_{1}\right) \exists \mathcal{K}_{1}>0$ and $\mathcal{L}_{1} \geq 0$ :

$$
\left|\left(F_{k}^{0}\left(x_{k}\right), \varphi_{n}\right)_{H}\right|+\left|\left(\partial_{n} F_{k}^{0}\left(x_{k}\right), \varphi_{n}\right)_{H}\right| \leq \mathcal{K}_{1} L_{k}\left(x_{k}\right)+\mathcal{L}_{1}
$$

$\left(\mathbb{L}_{2}\right) \exists \mathcal{K}_{2}>0$ and $\mathcal{L}_{2} \geq 0: \quad\left|\left(\partial_{n} F_{k}^{0}\left(x_{k}\right), x_{k}\right)_{H}\right| \leq \mathcal{K}_{2} L_{k}\left(x_{k}\right)+\mathcal{L}_{2} ;$

$\left(\mathbb{L}_{3}\right) \exists \mathcal{K}_{3}>0$ and $\mathcal{L}_{3} \geq 0: \quad\left|x_{k}\right|_{B}^{R} \leq \mathcal{K}_{3} L_{k}\left(x_{k}\right)+\mathcal{L}_{3}$.

If, in addition, the following two relations between the parameters are satisfied:

$$
\begin{gathered}
\Xi_{0}:=\mathcal{K}_{1} \operatorname{Tr}_{H} A^{-1}<1, \\
\Theta_{0}:=\|\mathcal{J}\| \cdot \mathcal{K}_{3} \frac{\mathcal{M}_{2}+\mathcal{M}_{1} \operatorname{Tr}_{H} A^{-1}}{1-\mathcal{K}_{1} \operatorname{Tr}_{H} A^{-1}}<1,
\end{gathered}
$$

then

$$
\sup _{\mu \in \mathcal{M}^{b}(\mathcal{X})} \sup _{k \in \mathbb{Z}} \int_{\mathcal{X}}\left|L_{k}\left(x_{k}\right)\right| d \mu(x) \leq \mathcal{C}_{1}^{\prime}<\infty
$$

and for all $Q \geq 1$,

$$
\begin{gathered}
\sup _{\mu \in \mathcal{M}^{b}\left(\mathcal{X}_{-}\right)} \sup _{k \in \mathbb{Z}} \int_{\mathcal{X}_{-}}\left|x_{k}\right|_{B}^{Q} d \mu(x) \leq \mathcal{C}_{Q}^{\prime \prime}<\infty, \\
\sup _{\mu \in \mathcal{M}^{b}\left(\mathcal{X}_{-}\right)} \sup _{k \in \mathbb{Z}} \int_{\mathcal{X}_{-}}\left(\sum_{j \in \mathbb{Z}} \mathcal{J}_{k, j}\left|x_{j}\right|_{B}^{R}\right)^{Q} d \mu(x) \leq \mathcal{C}_{Q}^{\prime \prime \prime}<\infty .
\end{gathered}
$$

If, in addition, for some $Q \geq 1$,

$$
\Xi_{Q-1}:=\mathcal{K}_{1}\left[1+(Q-1) \mathcal{K}_{2}\right] \operatorname{Tr}_{H} A^{-1}<1,
$$


then

$$
\sup _{\mu \in \mathcal{M}^{b}\left(\mathcal{X}_{-}\right)} \sup _{k \in \mathbb{Z}} \int_{\mathcal{X}_{-}}\left|L_{k}\left(x_{k}\right)\right|^{Q} d \mu(x) \leq \mathcal{C}_{Q}^{\prime}<\infty .
$$

Proof. To get the estimates (7.21)-(7.23) which are uniform in $k \in \mathbb{Z}$, in view of (7.6)(7.9) we can endow the space $\mathcal{X}:=l^{2}\left(\mathbb{Z} \rightarrow B ; \gamma^{-1}\right) \cap X^{\mathbb{Z}} \subset l^{R}\left(\mathbb{Z} \rightarrow B ; \gamma^{-R}\right)$ with the family of (mutually equivalent) norms $\|\cdot\|_{R, k_{0}}, k_{0} \in \mathbb{Z}$,

$$
\begin{aligned}
\|x\|_{R, k_{0}}:=\left\|T_{k_{0}} x\right\|_{l^{R}\left(\gamma^{-R}\right)} & :=\left[\sum_{k \in \mathbb{Z}} \gamma_{k-k_{0}}^{-R}\left|x_{k}\right|_{B}^{R}\right]^{1 / R} \\
& \leq \gamma_{j}\|x\|_{l^{R}\left(\gamma^{-R}\right)} \leq \gamma_{j}\left\|O^{ \pm}\right\|_{H S}\|x\|_{\mathcal{B}} \leq \gamma_{j}\|x\|_{\mathcal{B}} .
\end{aligned}
$$

An important observation is that the matrix $\mathcal{J}$ is uniformly bounded in all the spaces $l^{1}\left(\mathbb{Z} ;\left\{\gamma_{k-k_{0}}^{-R}\right\}_{k \in \mathbb{Z}}\right)$, that is,

$$
\|\mathcal{J}\|_{k_{0}}:=\sup _{k \in \mathbb{Z}}\left\{\sum_{j \in \mathbb{Z}} \mathcal{J}_{k, j} \gamma_{k-k_{0}}^{-R} \gamma_{j-k_{0}}^{R}\right\} \leq \sup _{k \in \mathbb{Z}} \sum_{j \in \mathbb{Z}} \mathcal{J}_{k, j} \gamma_{k-j}^{R}=\|\mathcal{J}\|<\infty .
$$

To prove the theorem, we perform induction on $Q$ and proceed in several steps.

Step 1. As already mentioned in (7.13), Assumptions $\left(\mathbb{L}_{1}\right),\left(\mathbb{L}_{2}\right)$ imply the uniform lower boundedness of $L_{k}$, i.e., that

$$
\inf _{k \in \mathbb{Z}} \inf _{x_{k} \in X} L_{k}\left(x_{k}\right) \geq-l:=-\min \left\{\mathcal{L}_{1} \mathcal{K}_{1}^{-1}, \mathcal{L}_{2} \mathcal{K}_{2}^{-1}\right\}>-\infty
$$

For the sake of convenience we introduce the following functionals on $\mathcal{X}$ :

$$
\begin{gathered}
1 \leq \tilde{L}_{k}(x):=L_{k}\left(x_{k}\right)+\left(G_{k}(x), x_{k}\right)_{H}+\mathcal{M}_{2} \sum_{j \in \mathbb{Z}} \mathcal{J}_{k, j}\left|x_{j}\right|_{B}^{R}+\mathcal{N}_{2}+l+1, \\
1 \leq Z_{Q, k}(x):=Z_{Q, k, \varepsilon, \varepsilon^{\prime}}(x):=1+\varepsilon^{\prime}\left(\left|F_{k}^{0}\left(x_{k}\right)\right|_{B^{*}}^{2 Q}+\left|x_{k}\right|_{B}^{2 Q R}\right)+\varepsilon\|x\|_{-}^{2 Q R}, \\
Z_{Q}(x):=1+\varepsilon\|x\|_{-}^{2 Q R},
\end{gathered}
$$

where $Q \geq 1, k \in \mathbb{Z}$ and $0<\varepsilon, \varepsilon^{\prime} \leq 1$. Fix any basis vector $h_{i}, i=(k, n)$ and integrate by parts along the direction $h_{i}$ the following two families of test functions on $\mathcal{X}$ simultaneously:

$$
\begin{gathered}
f_{i}(x):=f_{Q, i, \varepsilon, \varepsilon^{\prime}}(x):=\left[\tilde{L}_{k}(x)\right]^{Q-1}\left(F_{k}(x), \varphi_{n}\right)_{H} Z_{Q, k, \varepsilon, \varepsilon^{\prime}}^{-1}(x), \\
g_{i}(x):=g_{Q^{\prime}, i, k_{0}, \varepsilon, \varepsilon^{\prime}}(x):=\|x\|_{R, k_{0}}^{R\left(Q^{\prime}-1\right)}\left(F_{k}(x), \varphi_{n}\right)_{H} Z_{Q^{\prime}, k, \varepsilon, \varepsilon^{\prime}}^{-1}(x),
\end{gathered}
$$

where $Q \geq 1, Q^{\prime}>2, k_{0} \in \mathbb{Z}$ and $0<\varepsilon, \varepsilon^{\prime} \leq 1$. Note that by construction $f_{i}, g_{i} \in C_{b}^{1}(\mathcal{X})$ satisfy the growth condition (7.17). Then elementary calculations (analogous to (6.16)(6.22)) show that for all $x \in \mathcal{X}$,

$$
\begin{aligned}
\partial_{i} f_{i}(x) \leq\left[\tilde{L}_{k}\right]^{Q-1}\left(\partial_{i} F_{k}, \varphi_{n}\right)_{H} Z_{Q, k}^{-1}+\left[\tilde{L}_{k}\right]^{Q-1}\left|\left(F_{k}, \varphi_{n}\right)_{H}\right| \cdot\left|Z_{Q, k}^{-2} \partial_{i} Z_{Q, k}\right| \\
+(Q-1)\left[\tilde{L}_{k}\right]^{Q-2}\left|\left(F_{k}, \varphi_{n}\right)_{H}\right| Z_{Q, k}^{-1}\left[\left(\partial_{i} F_{k}, x_{k}\right)_{H}+\left(F_{k}, \varphi_{n}\right)_{H}\right. \\
\left.+R \mathcal{M}_{2} \sum_{j \in \mathbb{Z}} \mathcal{J}_{k, j}\left|x_{j}\right|_{B}^{R-1}\left|\varphi_{n}\right|_{B}\right]
\end{aligned}
$$

and, respectively,

$$
\begin{aligned}
\partial_{i} g_{i}(x) \leq & \|x\|_{R, k_{0}}^{R\left(Q^{\prime}-1\right)}\left(\partial_{i} F_{k}, \varphi_{n}\right)_{H} Z_{Q^{\prime}, k}^{-1}+\|x\|_{R, k_{0}}^{R\left(Q^{\prime}-1\right)}\left|\left(F_{k}, \varphi_{n}\right)_{H}\right| \cdot\left|Z_{Q^{\prime}, k}^{-2} \partial_{i} Z_{Q^{\prime}, k}\right| \\
& +R\left(Q^{\prime}-1\right)\|x\|_{R, k_{0}}^{R\left(Q^{\prime}-1\right)-1}\left\|h_{i}\right\|_{R, k_{0}}\left|\left(F_{k}, \varphi_{n}\right)\right|_{H} Z_{Q^{\prime}, k}^{-1}
\end{aligned}
$$


with the uniform bound

$$
\begin{aligned}
\left|Z_{Q, k}^{-1} \partial_{i} Z_{Q, k}\right| & \leq 2 Q \frac{\varepsilon^{\prime}\left(\left|F_{k}^{0}\right|_{B^{*}}^{2 Q-1}\left|\partial_{n} F_{k}^{0}\right|_{B^{*}}+R\left|x_{k}\right|_{B}^{2 Q R-1}\left|\varphi_{n}\right|_{B}\right)+\varepsilon\left|\varphi_{n}\right|_{B} R\|x\|_{\mathcal{H}}^{2 Q R-1}}{1+\varepsilon^{\prime}\left(\left|F_{k}^{0}\right|_{B^{*}}^{2 Q}+\left|x_{k}\right|_{B}^{2 Q R}\right)+\varepsilon\|x\|_{\mathcal{H}}^{2 Q R}} \\
& \leq 2 Q\left[2 \mathcal{K}_{0}+\left(\varepsilon^{\prime}\right)^{\frac{1}{2 Q}}\left(\mathcal{L}_{0}+\kappa\right)+\varepsilon^{\frac{1}{2 Q R}} R \kappa\right] \\
& =: \mathcal{Z}_{Q, \varepsilon, \varepsilon^{\prime}}^{\prime}=: \mathcal{Z}_{Q}^{\prime},\left.\quad \mathcal{Z}_{Q, \varepsilon, \varepsilon^{\prime}}^{\prime}\right|_{\varepsilon^{\prime}=0}=: \mathcal{Z}_{Q} .
\end{aligned}
$$

Thus,

$$
\begin{aligned}
\partial_{i} f_{i} \leq & {\left[\tilde{L}_{k}\right]^{Q-2}\left[\tilde{L}_{k}+(Q-1) \hat{L}_{k, 2}\right] \Gamma_{k, 1} Z_{Q, k}^{-1} } \\
& +\left[\tilde{L}_{k}\right]^{Q-2}\left|\left(F_{k}, \varphi_{n}\right)_{H}\right| \cdot\left[\mathcal{Z}_{Q}^{\prime} \tilde{L}_{k}+(Q-1) \Gamma_{k, 1}\right] Z_{Q, k}^{-1}
\end{aligned}
$$

and, respectively,

$$
\begin{aligned}
\partial_{i} g_{i} \leq & \|x\|_{R, k_{0}}^{R\left(Q^{\prime}-1\right)-1}\left[\|x\|_{R, k_{0}}+R\left(Q^{\prime}-1\right) \gamma_{k-k_{0}}^{-2} \kappa\right] \Gamma_{k, 1} Z_{Q^{\prime}, k}^{-1} \\
& +\mathcal{Z}_{Q^{\prime}}^{\prime}\|x\|_{R, k_{0}}^{R\left(Q^{\prime}-1\right)} \cdot\left|\left(F_{k}, \varphi_{n}\right)_{H}\right| Z_{Q^{\prime}, k}^{-1},
\end{aligned}
$$

where we denote

$$
\begin{aligned}
& \Gamma_{k, 1}(x):=\mathcal{K}_{1} L_{k}\left(x_{k}\right)+\mathcal{M}_{1} \sum_{j \in \mathbb{Z}} \mathcal{J}_{k, j}\left|x_{j}\right|_{B}^{R}+\mathcal{L}_{1}+\mathcal{N}_{1}, \\
& \Gamma_{k, 2}(x):=\mathcal{K}_{2} L_{k}\left(x_{k}\right)+\mathcal{M}_{2}(1+\kappa R) \sum_{j \in \mathbb{Z}} \mathcal{J}_{k, j}\left|x_{j}\right|_{B}^{R}+\mathcal{L}_{2}+\mathcal{N}_{2} .
\end{aligned}
$$

On the other hand, by the (IbP)-formula (7.16),

$$
\begin{aligned}
& \int_{\mathcal{X}}\left[\tilde{L}_{k}(x)\right]^{Q-1}\left(F_{k}(x), \varphi_{n}\right)_{H}\left(x_{k}, \varphi_{n}\right)_{H} Z_{Q, k}^{-1}(x) d \mu(x) \\
& \quad=\lambda_{n}^{-1} \int_{\mathcal{X}}\left\{\partial_{i} f(x)-\left[\tilde{L}_{k}(x)\right]^{Q-1}\left(F_{k}(x), \varphi_{n}\right)_{H}^{2} Z_{Q, k}^{-1}(x)\right\} d \mu(x)
\end{aligned}
$$

and, respectively,

$$
\begin{aligned}
\int_{\mathcal{X}} \| & x \|_{R, k_{0}}^{R\left(Q^{\prime}-1\right)}\left(F_{k}(x), \varphi_{n}\right)_{H}\left(x_{k}, \varphi_{n}\right)_{H} Z_{Q^{\prime}, k}^{-1}(x) d \mu(x) \\
& =\lambda_{n}^{-1} \int_{\mathcal{X}}\left\{\partial_{i} g(x)-\|x\|_{R, k_{0}}^{R\left(Q^{\prime}-1\right)}\left(F_{k}(x), \varphi_{n}\right)_{H}^{2} Z_{Q^{\prime}, k}^{-1}(x)\right\} d \mu(x) .
\end{aligned}
$$

Taking the sum of the inequalities (7.38) and (7.39) each over $n \in \mathbb{N}$ and using the same arguments as in the proof of Theorem 6.2, one arrives at the estimates

$$
\begin{aligned}
\int_{\mathcal{X}}\left[\tilde{L}_{k}(x)\right]^{Q} Z_{Q, k}^{-1}(x) d \mu(x) \\
\leq \operatorname{Tr}_{H} A^{-1} \sup _{n \in \mathbb{N}} \int_{\mathcal{X}}\left\{\partial_{i} f(x)-\left[\tilde{L}_{k}(x)\right]^{Q-1}\left(F_{k}(x), \varphi_{n}\right)_{H}^{2} Z_{Q, k}^{-1}(x)\right\} d \mu(x) \\
\left.\quad+\int_{\mathcal{X}}\left[\tilde{L}_{k}(x)\right]^{Q-1}\left[\mathcal{M}_{2} \sum_{j \in \mathbb{Z}} \mathcal{J}_{k, j}\left|x_{j}\right|_{B}^{R}+\mathcal{N}_{2}+l+1\right)\right] Z_{Q, k}^{-1}(x) d \mu(x)
\end{aligned}
$$


and, respectively,

$$
\begin{aligned}
& \int_{\mathcal{X}}\|x\|_{R, k_{0}}^{R\left(Q^{\prime}-1\right)} L_{k}\left(x_{k}\right) Z_{Q^{\prime}, k}^{-1}(x) d \mu(x) \\
& \leq \operatorname{Tr}_{H} A^{-1} \sup _{n \in \mathbb{N}} \int_{\mathcal{X}}\left\{\partial_{i} g(x)-\|x\|_{R, k_{0}}^{R\left(Q^{\prime}-1\right)}\left(F_{k}(x), \varphi_{n}\right)_{H}^{2} Z_{Q^{\prime}, k}^{-1}(x)\right\} d \mu(x) \\
&+\int_{\mathcal{X}}\|x\|_{R, k_{0}}^{R\left(Q^{\prime}-1\right)}\left[\mathcal{M}_{2} \sum_{j \in \mathbb{N}} \mathcal{J}_{k, j}\left|x_{j}\right|_{B}^{R}+\mathcal{N}_{2}+l+1\right] Z_{Q^{\prime}, k}^{-1}(x) d \mu(x) .
\end{aligned}
$$

Step 2: $Q=1$. We have from (7.35) and (7.40) that more precisely

$$
\begin{aligned}
\int_{\mathcal{X}}[ & \left.L_{k}\left(x_{k}\right)+\left(G_{k}(x), x_{k}\right)_{H}\right] Z_{1, k}^{-1}(x) d \mu(x) \\
\leq & \operatorname{Tr}_{H} A^{-1} \int_{\mathcal{X}} \Gamma_{k, 1}(x) Z_{1, k}^{-1}(x) d \mu(x) \\
& \quad+\operatorname{Tr}_{H} A^{-1} \sup _{n \in \mathbb{N}} \int_{\mathcal{X}}\left\{\mathcal{Z}_{1}^{\prime}\left|\left(F_{k}(x), \varphi_{n}\right)_{H}\right|-\left(F_{k}(x), \varphi_{n}\right)_{H}^{2}\right\} Z_{1, k}^{-1}(x) d \mu(x)
\end{aligned}
$$

and furthermore due to $\left(\mathbb{G}_{2}\right),\left(\mathbb{L}_{1}\right)$ and $\left(\mathbb{L}_{3}\right)$,

$$
\begin{aligned}
\int_{\mathcal{X}}\left|x_{k}\right|_{B}^{R} Z_{1, k}^{-1}(x) d \mu(x)-\mathcal{L}_{3} \\
\leq \int_{\mathcal{X}} \mathcal{K}_{3} L_{k}\left(x_{k}\right) Z_{1, k}^{-1}(x) d \mu(x) \\
\leq \mathcal{K}_{3} \frac{\mathcal{M}_{2}+\mathcal{M}_{1} \operatorname{Tr}_{H} A^{-1}}{1-\Xi_{0}} \int_{\mathcal{X}}\left[\sum_{j \in \mathbb{Z}} \mathcal{J}_{k, j}\left|x_{j}\right|_{B}^{R}\right] Z_{1, k}^{-1}(x) d \mu(x) \\
\quad+\mathcal{K}_{3} \frac{\mathcal{N}_{2}+\left(\mathcal{L}_{1}+\mathcal{N}_{1}+\frac{1}{4}\left[\mathcal{Z}_{1}^{\prime}\right]^{2}\right) \operatorname{Tr}_{H} A^{-1}}{1-\Xi_{0}}
\end{aligned}
$$

Letting $\varepsilon^{\prime} \searrow 0$ in (7.43) and then taking the sum over $k \in \mathbb{Z}$ with the weights $\gamma_{k-k_{0}}^{-R}$, by (7.27) and Lebesgue's dominated convergence theorem we get that

$$
\begin{aligned}
& \int_{\mathcal{X}}\|x\|_{R, k_{0}}^{R}(x) Z_{1}^{-1} d \mu(x) \\
& \quad \leq\left(1-\Theta_{0}\right)^{-1}\left(1-\Xi_{0}\right)^{-1}\left\|O^{ \pm}\right\|_{H S}^{2} \mathcal{K}_{3}\left\{\mathcal{N}_{2}+\left(\mathcal{L}_{1}+\mathcal{N}_{1}+\frac{1}{4}\left[\mathcal{Z}_{1}\right]^{2}\right) \operatorname{Tr}_{H} A^{-1}+\mathcal{K}_{3}^{-1} \mathcal{L}_{3}\right\} .
\end{aligned}
$$

Note that in doing so we used that by $(7.5)$,

$$
\sum_{k \in \mathbb{Z}} \gamma_{k-k_{0}}^{-R} \leq \sum_{k \in \mathbb{Z}} \gamma_{k}^{-1}=\left\|O^{ \pm}\right\|_{H S}^{2}
$$

and respectively by $(7.20)$,

$$
0 \leq \Xi_{0}:=\mathcal{K}_{1} \operatorname{Tr}_{H} A^{-1}<1, \quad 0 \leq \Theta_{0}:=\|\mathcal{J}\| \cdot \mathcal{K}_{3} \frac{\mathcal{M}_{2}+\mathcal{M}_{1} \operatorname{Tr}_{H} A^{-1}}{1-\Xi_{0}}<1 .
$$

Herewith, letting $\varepsilon \searrow 0$, by Fatou's lemma we obtain that

$$
\sup _{k_{0} \in \mathbb{Z}} \int_{\mathcal{X}}\|x\|_{R, k_{0}}^{R}(x) d \mu(x) \leq \mathcal{C}_{R}<\infty
$$


with a constant $\mathcal{C}_{R}:=\mathcal{C}_{R}\left(\mathcal{K}_{0}, \mathcal{K}_{1}, \ldots, \mathcal{N}_{2}\right)$ which equals the RHS in (7.44) with $\mathcal{Z}_{1}:=$ $\mathcal{Z}_{1, \varepsilon=0}=4 \mathcal{K}_{0}$. Hence due to (7.26) and (7.27),

$$
\begin{gathered}
\sup _{\mu \in \mathcal{M}^{b}(\mathcal{X})} \sup _{k \in \mathbb{Z}} \int_{\mathcal{X}}\left|x_{k}\right|_{B}^{R} d \mu(x) \leq \mathcal{C}_{R}^{\prime \prime}:=\gamma_{0}^{R} \mathcal{C}_{R}<\infty, \\
\sup _{\mu \in \mathcal{M}^{b}(\mathcal{X})} \sup _{k \in \mathbb{Z}} \int_{\mathcal{X}} \sum_{j \in \mathbb{Z}} \mathcal{J}_{k, j}\left|x_{j}\right|_{B}^{R} d \mu(x) \leq \mathcal{C}_{1}^{\prime \prime \prime}:=\|\mathcal{J}\| \cdot \gamma_{0}^{R} \mathcal{C}_{R}<\infty .
\end{gathered}
$$

From (7.43) and (7.48) by Fatou's lemma we conclude in turn that also

$$
\sup _{\mu \in \mathcal{M}^{b}(\mathcal{X})} \sup _{k \in \mathbb{Z}} \int_{\mathcal{X}}\left|L_{k}\left(x_{k}\right)\right| d \mu(x) \leq \mathcal{C}_{1}^{\prime}<\infty .
$$

Step 3: $Q^{\prime}>2$. Now we return to the general case of $Q^{\prime}>2$. From (7.36), (7.41) and Young's inequality it readily follows that for every $k \in \mathbb{N}$ and $0<\delta, \varepsilon, \varepsilon^{\prime} \leq 1$,

$$
\begin{aligned}
\int_{\mathcal{X}}\left|x_{k}\right|_{B}^{R}\|x\|_{R, k_{0}}^{R\left(Q^{\prime}-1\right)} Z_{Q^{\prime}, k}^{-1}(x) d \mu(x)-\mathcal{L}_{3} \\
\leq \mathcal{K}_{3} \int_{\mathcal{X}} L_{k}\left(x_{k}\right)\|x\|_{R, k_{0}}^{R\left(Q^{\prime}-1\right)} Z_{Q^{\prime}, k}^{-1}(x) d \mu(x) \\
\leq \mathcal{K}_{3} \frac{\mathcal{M}_{2}+\mathcal{M}_{1} T r_{H} A^{-1}+\delta}{1-\Xi_{0}-\delta} \int_{\mathcal{X}}\|x\|_{R, k_{0}}^{R\left(Q^{\prime}-1\right)} \sum_{j \in \mathbb{Z}} \mathcal{J}_{k, j}\left|x_{j}\right|_{B}^{R} Z_{Q^{\prime}, k}^{-1}(x) d \mu(x) \\
\quad+C_{Q^{\prime}}^{\prime} \int_{\mathcal{X}}\left[\|x\|_{R, k_{0}}^{R\left(Q^{\prime}-1\right)}+L_{k}\left(x_{k}\right)+\sum_{j \in \mathbb{Z}} \mathcal{J}_{k, j}\left|x_{j}\right|_{B}^{R}+1\right] Z_{Q^{\prime}, k}^{-1}(x) d \mu(x) .
\end{aligned}
$$

with $C_{Q^{\prime}}^{\prime}:=C_{Q^{\prime}}\left(\mathcal{K}_{1}, \ldots, \mathcal{N}_{2} ; \delta, \mathcal{Z}_{Q^{\prime}}^{\prime}\right) \in(0, \infty)$ continuously depending, among the other parameters, on $Q^{\prime} \geq 2$. Letting $\varepsilon^{\prime} \searrow 0$ (and hence $Z_{Q^{\prime}, k} \searrow Z_{Q^{\prime}}$ ) in (7.50) and then summing over $k \in \mathbb{Z}$ with the weights $\gamma_{k-k_{0}}^{-R}$, by (7.27) and Lebesgue's convergence theorem we get that for all $Q^{\prime} \geq 2$,

$$
\begin{aligned}
& {\left[1-\|\mathcal{J}\| \cdot \mathcal{K}_{3} \frac{\mathcal{M}_{2}+\mathcal{M}_{1} \operatorname{Tr}_{H} A^{-1}+\delta}{1-\Xi_{0}-\delta}\right] \int_{\mathcal{X}}\|x\|_{R, k_{0}}^{R Q^{\prime}} Z_{Q^{\prime}}^{-1}(x) d \mu(x)} \\
& \quad \leq C_{Q^{\prime}}^{\prime \prime} \int_{\mathcal{X}}\left[\|x\|_{R, k_{0}}^{R\left(Q^{\prime}-1\right)}+1\right] Z_{Q^{\prime}}^{-1}(x) d \mu(x)
\end{aligned}
$$

with some new constant $C_{Q^{\prime}}^{\prime \prime}:=C_{Q^{\prime}}\left(\mathcal{K}_{1}, \ldots, \mathcal{N}_{2} ; \delta, \mathcal{Z}_{Q^{\prime}}^{\prime}\right) \in(0, \infty)$ (even though $\|x\|_{R, k_{0}}$ is not differentiable at $x=0$ and we cannot directly apply the (IbP)-formula (7.16) when $\left.Q^{\prime}=2\right)$. Note that in doing so we took into account the estimates (7.47)-(7.49) proved above. Suppose that we already know that

$$
\sup _{k_{0} \in \mathbb{Z}} \int_{\mathcal{X}}\|x\|_{R, k_{0}}^{R\left(Q^{\prime}-1\right)}(x) d \mu(x) \leq \mathcal{C}_{R\left(Q^{\prime}-1\right)}<\infty
$$

(as was the case for $Q^{\prime}=2$ in (7.46)). Fix $\delta>0$ small enough so that

$$
\Theta_{0}<\Theta_{\delta}:=\|\mathcal{J} \mid\| \cdot \mathcal{K}_{3} \frac{\mathcal{M}_{2}+\mathcal{M}_{1} \operatorname{Tr}_{H} A^{-1}+\delta}{1-\Xi_{0}-\delta}<1 .
$$

Then by Fatou's lemma, letting $\varepsilon \searrow 0$ (and thus $Z_{Q^{\prime}} \searrow 1$ ) in (7.51), we obtain that

$$
\sup _{k_{0} \in \mathbb{Z}} \int_{\mathcal{X}}\|x\|_{R, k_{0}}^{R Q^{\prime}} d \mu(x) \leq \mathcal{C}_{R, Q^{\prime}}<\infty
$$


with a proper constant $\mathcal{C}_{R, Q^{\prime}}:=\mathcal{C}_{R, Q^{\prime}}\left(\mathcal{K}_{0}, \mathcal{K}_{1}, \ldots, \mathcal{N}_{2}\right)$. So, by induction, the estimate (7.53) is valid for all $Q^{\prime} \geq 2$. Since

$$
\sum_{j \in \mathbb{Z}} \mathcal{J}_{k, j}\left|x_{j}\right|_{B}^{R} \leq\|\mid \mathcal{J}\| \cdot\|x\|_{R, k}^{R}
$$

(7.53) immediately implies the desired estimates (7.22) and (7.23).

Step 4: $Q>1$. A similar reasoning as in the proof of Theorem 6.2 shows by (7.35), (7.40) and Young's inequality that for every $k \in \mathbb{Z}$ and $0<\delta, \varepsilon, \varepsilon^{\prime} \leq 1$,

$$
\begin{gathered}
\left\{1-\mathcal{K}_{1}\left[1+(Q-1) \mathcal{K}_{2}\right] \operatorname{Tr}_{H} A^{-1}-\delta\right\} \int_{\mathcal{X}}\left[\tilde{L}_{k}\left(x_{k}\right)\right]^{Q} Z_{Q, k}^{-1}(x) d \mu(x) \\
\leq C_{Q} \int_{\mathcal{X}}\left[\tilde{L}_{k}\left(x_{k}\right)\right]^{Q-1}\left(\sum_{j \in \mathbb{Z}} \mathcal{J}_{k, j}\left|x_{j}\right|_{B}^{R}+1\right) Z_{Q, k}^{-1}(x) d \mu(x)
\end{gathered}
$$

with some constant $C_{Q}:=C_{Q}\left(\mathcal{K}_{1}, \ldots, \mathcal{N}_{2} ; \delta, \mathcal{Z}_{Q, \varepsilon, \varepsilon^{\prime}}^{\prime}\right) \in(0, \infty)$. If $1<Q \leq 2$ and thus $0<\left[\tilde{L}_{k}\left(x_{k}\right)\right]^{Q-2} \leq 1$, we continue the estimate (7.54) in a trivial way to

$$
\begin{aligned}
(1- & \left.\Xi_{Q-1}-\delta\right) \int_{\mathcal{X}}\left[\tilde{L}_{k}\left(x_{k}\right)\right]^{Q} Z_{Q, k}^{-1}(x) d \mu(x) \\
& \leq C_{Q}\left\{\int_{\mathcal{X}}\left(\sum_{j \in \mathbb{Z}} \mathcal{J}_{k, j}\left|x_{j}\right|_{B}^{R}\right) d \mu(x)+1\right\},
\end{aligned}
$$

or, if otherwise $Q>2$, then respectively by Hölder's inequality to

$$
\begin{aligned}
& \left(1-\Xi_{Q-1}-\delta\right)\left(\int_{\mathcal{X}}\left[\tilde{L}_{k}\right]^{Q} Z_{Q, k}^{-1} d \mu\right)^{\frac{1}{Q}} \\
& \quad \leq C_{Q}\left\{\left(\int_{\mathcal{X}}\left(\sum_{j \in \mathbb{Z}} \mathcal{J}_{k, j}\left|x_{j}\right|_{B}^{R}\right)^{Q} d \mu(x)\right)^{\frac{1}{Q}}+1\right\} .
\end{aligned}
$$

Fix $\delta>0$ small enough so that $\Xi_{Q-1}+\delta<1$. Then by Fatou's lemma, letting $\varepsilon, \varepsilon^{\prime} \searrow 0$ and hence $Z_{Q, k} \searrow 1$, we conclude from (7.23), (7.55) and (7.56) that

$$
\sup _{\mu \in \mathcal{M}^{b}(\mathcal{X})} \sup _{k \in \mathbb{Z}} \int_{\mathcal{X}}\left[\tilde{L}_{k}\left(x_{k}\right)\right]^{Q}(x) d \mu(x) \leq \tilde{C}_{Q}<\infty .
$$

Thus

$$
\sup _{\mu \in \mathcal{M}^{b}(\mathcal{X})} \sup _{k \in \mathbb{Z}} \int_{\mathcal{X}}\left|L_{k}\left(x_{k}\right)\right|^{Q} d \mu(x) \leq \mathcal{C}_{Q}^{\prime}<\infty,
$$

which completes the proof of Theorem 7.1. Finally we note that of course all the constants in the above estimates (i.e., $\left.\mathcal{C}_{Q}^{\prime}, \mathcal{C}_{Q}^{\prime \prime}, \mathcal{C}_{Q}^{\prime \prime \prime}\right)$ can be calculated explicitly when needed.

Moreover, let us suppose that the functionals $L_{k}$ satisfy additionally the standard coercivity property:

$\left(\mathbb{L}_{4}\right) \forall \mathcal{K}_{4}>0 \exists \mathcal{L}_{4} \geq 0: \quad\left|x_{k}\right|_{H}^{2} \leq \mathcal{K}_{4} L_{k}\left(x_{k}\right)+\mathcal{L}_{4}$ uniformly for all $x_{k} \in X$ and $k \in \mathbb{Z}$.

As in Subsect. 6.2, we write the decomposition of $b$ with arbitrary $a \in \mathbb{R}$ as

$$
\begin{gathered}
b_{i}(x)=b_{(k, n)}(x):=-\left(\tilde{A} \varphi_{n}, x_{k}\right)_{H}-\left(\tilde{F}_{k}^{0}\left(x_{k}\right), \varphi_{n}\right)_{H}-\left(G_{k}(x), \varphi_{n}\right)_{H}, \\
\text { where } \tilde{A}:=A+a^{2} \mathbf{1}, \quad \tilde{F}_{k}^{0}\left(x_{k}\right):=F_{k}^{0}\left(x_{k}\right)-a^{2} x_{k}, \\
i=(k, n) \in \mathbb{Z} \times \mathbb{N}, x \in \mathcal{X} .
\end{gathered}
$$


Then choosing $a^{2} \geq a^{2}(Q, \varepsilon)>0$ large enough, we can always achieve that $\operatorname{Tr}_{H} \tilde{A}^{-1}<\varepsilon$ and $\Xi_{Q-1}<\varepsilon$ for any given $\varepsilon>0$. Analogously, by (7.29) for any $\delta>0$ and $0<\mathcal{K}_{4}<$ $\delta a^{-2}$,

$$
\tilde{L}_{k}\left(x_{k}\right):=\left(\tilde{F}_{k}^{0}\left(x_{k}\right), x_{k}\right)_{H} \geq(1-\delta) L_{k}\left(x_{k}\right)-\delta \mathcal{L}_{4} \mathcal{K}_{4}^{-1}
$$

and thus all $\tilde{L}_{k}$ satisfy $\left(\mathbb{L}_{3}\right)$ with the same constant $\tilde{\mathcal{K}}_{3}=\mathcal{K}_{3}(1-\delta)^{-1}$. Therefore, instead of $\Theta_{0}<1$ in the formulation of Theorem 7.1, it suffices to assume that $\mathcal{K}_{3} \mathcal{M}_{2} \cdot\|\mathcal{J}\|<1$. Altogether this gives the following modification of Theorem 7.1.

Theorem 7.1' (Refinement of Theorem 7.1). Let Assumptions $(\mathbb{J}),\left(\mathbb{F}_{0}\right),\left(\mathbb{G}_{1,2}\right)$ and $\left(\mathbb{L}_{1-4}\right)$ hold. Then the moment estimates (7.22), (7.23) and (7.25) are satisfied for all $Q \geq 1$ provided

$$
\Theta_{0}^{\prime}:=\mathcal{K}_{3} \mathcal{M}_{2} \cdot\|\mathcal{J} \mid\|<1
$$

7.2.2. Integrability of logarithmic derivatives and Sobolev norms. The next statement gives a corresponding generalization of Corollary 6.3. It follows immediately from the proof of our main Theorem 7.1.

Corollary 7.2 (Integrability of Logarithmic Derivatives). (i) Suppose that under the assumptions of Theorem 7.1, additionally,

$$
\Theta_{0}<1 \text { and } \Xi_{Q-1}<1 \text { for some } Q \geq 1 \text {. }
$$

Then

$$
\sup _{\mu \in \mathcal{M}^{b}(\mathcal{X})} \sup _{k \in \mathbb{Z}} \int_{\mathcal{X}}\left|b_{(k, n)}\right|^{Q} d \mu \leq \mathcal{C}_{Q, n}<\infty, \quad n \in \mathbb{N},
$$

and hence the (IbP)-formula (7.16) can be extended to all $f \in C_{b, l o c}^{1}(\mathcal{X})$ satisfying the polynomial growth condition: for each $n \in \mathbb{N}$ there exist $C:=C(f, k, n)>0$ and $Q:=Q(f, k, n) \geq 1$ such that for all $x \in \mathcal{X}$,

$$
|f(x)|+\left|\partial_{(k, n)} f(x)\right| \leq C\left(1+|x|_{l^{R}\left(\mathbb{Z} \rightarrow B ; \gamma^{-1}\right)}\right)^{Q} .
$$

(ii) If, moreover, the partial logarithmic derivatives $b_{(k, n)}$ (or, even, the coercivity functionals $L_{k}$ ) have at most polynomial growth at infinity, i.e., $\exists \mathcal{K}_{n}^{\prime}, \mathcal{L}_{n}^{\prime}, R_{n}^{\prime}>0$ (resp. $\left.\mathcal{K}^{\prime}, \mathcal{L}^{\prime}, R^{\prime}>0\right)$ such that $\forall x \in \mathcal{X}$

$$
\begin{gathered}
\left|b_{(k, n)}(x)\right| \leq \mathcal{K}_{n}^{\prime}\left|x_{k}\right|_{B}^{R_{n}^{\prime}}+\mathcal{L}_{n}^{\prime}+\mathcal{M}_{1} \sum_{j \in \mathbb{Z}} \mathcal{J}_{k, j}\left|x_{j}\right|_{B}^{R}+\mathcal{N}_{1} \\
\left(\text { resp. }\left|L_{k}(x)\right| \leq \mathcal{K}^{\prime}\left|x_{k}\right|_{B}^{R^{\prime}}+\mathcal{L}^{\prime}+\mathcal{M}_{2} \sum_{j \in \mathbb{Z}} \mathcal{J}_{k, j}\left|x_{j}\right|_{B}^{R}+\mathcal{N}_{2}\right),
\end{gathered}
$$

then (7.57) (or, even stronger, the estimate

$$
\left.\sup _{\mu \in \mathcal{M}^{b}(\mathcal{X})} \sup _{k \in \mathbb{Z}} \int_{\mathcal{X}}\left|L_{k}\left(x_{k}\right)\right|^{Q} d \mu(x)<\infty\right)
$$

holds for all $Q \geq 1$.

The next two statements give corresponding generalizations of Theorems 6.7 and $6.7^{\prime}$.

Theorem 7.3 (A Priori Moment Estimates for Sobolev Norms). Suppose that under the assumptions of Theorem 7.1, additionally,

$$
\Theta_{0}<1 \text { and } \Xi_{2 Q-1}<1 \text { for some given } Q \geq 1 .
$$

Furthermore, assume that the linear part of the logarithmic derivative $b$ satisfies:

$\left(\mathbb{T}_{\alpha}\right) \operatorname{Tr}_{H} A^{\alpha-1}<\infty$ for some $\alpha \geq 0$. 
Then

$$
\mu\left(\mathcal{X} \cap l^{2}\left(\mathbb{Z} \rightarrow H^{\alpha} ; \gamma^{-1}\right)\right)=1
$$

and, moreover,

$$
\sup _{\mu \in \mathcal{M}^{b}(\mathcal{X})} \sup _{k \in \mathbb{Z}} \int_{\mathcal{X}_{-}}\left|x_{k}\right|_{H^{\alpha}}^{Q+1} d \mu \leq C_{Q+1, \alpha}<\infty .
$$

Proof. This proof is completely similar to the proof of Theorem 6.5. Due to Corollary 7.2 one can apply the (IbP)-formula (7.16) in the given direction $h_{i} \in \operatorname{bas}(\mathcal{H}), i=(k, n) \in$ $\mathbb{Z}^{d+1}$, to the following cylinder functions:

$$
\begin{gathered}
g_{K, n}(x):=g_{K, n, k}\left(x_{k}\right):=\left(x_{k}, \varphi_{n}\right)_{H}\left|P_{K} x_{k}\right|_{H^{\alpha}}^{Q-1}, \\
\left|g_{K, n}(x)\right| \leq\left|P_{K} x_{k}\right|_{H^{\alpha}}^{Q}, \quad\left|\partial_{(k, n)} g_{k, n}(x)\right| \leq Q\left|P_{K} x_{k}\right|_{H^{\alpha}}^{Q-1}, \quad x \in \mathcal{X}_{-},
\end{gathered}
$$

where $Q>2, k \in \mathbb{Z}$ and $K \geq n$. Thus, as already calculated in (6.50) and (6.51), we get

$$
\begin{aligned}
\lambda_{n} \int_{\mathcal{X}}\left(x_{k}, \varphi_{n}\right)^{2}\left|P_{K} x_{k}\right|_{H^{\alpha}}^{Q-1} d \mu(x) \\
\quad \leq \int_{\mathcal{X}}\left[\left|P_{K} x\right|_{H^{\alpha}}^{Q}+Q\left|P_{K} x\right|_{H^{\alpha}}^{Q-1}\right] d \mu(x) \\
\quad+\sup _{n \in \mathbb{N}} \int_{\mathcal{X}}\left|\left(F_{k}\left(x_{k}\right)+G_{k}(x), \varphi_{n}\right)_{H}\left(x_{k}, \varphi_{n}\right)_{H}\right|^{Q} d \mu(x) .
\end{aligned}
$$

Herewith, summing over $1 \leq n \leq K$ and using $\left(\mathbb{L}_{1}^{\prime}\right),\left(\mathbb{G}_{1}\right),(7.23)$ and (7.25), we conclude that uniformly for all $K \in \mathbb{N}$,

$$
\begin{aligned}
& \left(1-\delta \operatorname{Tr}_{H} A^{\alpha-1}\right) \int_{\mathcal{X}}\left|P_{K} x_{k}\right|_{H^{\alpha}}^{Q+1} d \mu(x) \\
& \quad \leq \operatorname{Tr}_{H} A^{\alpha-1}\left\{\int_{\mathcal{X}}\left(\mathcal{K}_{1} L_{H}^{F}(x)+\mathcal{M}_{1} \sum_{j \in \mathbb{Z}} \mathcal{J}_{k, j}\left|x_{j}\right|_{B}^{R}+\mathcal{L}_{1}+\mathcal{N}_{1}\right)^{2 Q} d \mu(x)+C(Q, \delta)\right\} \\
& \quad<\infty
\end{aligned}
$$

as soon as $0<\delta<\left(\operatorname{Tr}_{H} A^{\alpha-1}\right)^{-1} \leq 1$. Finally, letting $K \rightarrow \infty$ in (7.65), from Fatou's lemma we obtain the desired estimates (7.63) and (7.64).

Theorem 7.3' (Refinement of Theorem 7.3). Let Assumptions $(\mathbb{J}),\left(\mathbb{F}_{0}\right),\left(\mathbb{G}_{1,2}\right),\left(\mathbb{L}_{1-4}\right)$ and $\left(\mathbb{T}_{\alpha}\right)$ hold. Then the moment estimates $(7.61)$ for the Sobolev norms $|\cdot|_{H^{\alpha}}$ are satisfied for all $Q \geq 1$ provided in (7.58), $\Theta_{0}^{\prime}<1$.

7.3. Applications to Euclidean Gibbs states: Proof of Hypotheses (H) and $\left(\mathbf{H}_{1 \mathrm{oc}}\right)$. Here we come back to the Euclidean Gibbs measures and, on the basis of the abstract Theorems $7.1^{\prime}$ and $7.3^{\prime}$, verify for them Hypotheses $(\mathbf{H})$ and $\left(\mathbf{H}_{l o c}\right)$, which were left as the crucial steps for our proof of Main Theorems I-III in Subsect. 5.2.

Theorem 7.4 (cf. Hypothesis (H): A Priori Estimates for Tempered Gibbs Measures on Lebesgue and Sobolev Loops). (i) Let Assumptions $(\mathbf{W}),(\mathbf{J})$ and $(\mathbf{V})$ on the interaction potentials of the general QLS (3.1) hold with some fixed, but small enough (e.g., satisfying (7.74) below)

$$
0<K_{3}<K_{3}^{0}\left(R,\|\mathbf{J}\|_{0}\right) .
$$

Then every $\mu \in \mathcal{G}_{t}:=\mathcal{G}_{(s) t}^{R}$ such that

$$
\mu\left(\Omega_{-p}^{R}\right)=1 \quad \text { for some } p=p(\mu)>d
$$


is actually supported by the set

$$
\bigcap_{0 \leq \alpha<1 / 2, p>d} l^{2}\left(\mathbb{Z}^{d} \rightarrow W_{\beta}^{2, \alpha} ; \gamma_{-p}\right)
$$

(where $\gamma_{p}=\left\{\gamma_{p, k}\right\}_{k \in \mathbb{Z}^{d}}$ is the weight sequence with $\gamma_{p, k}:=(1+|k|)^{p}$ and $W_{\beta}^{2, \alpha}$ is the space of Sobolev loops with the norm $\left\|\omega_{k}\right\|_{W_{\beta}^{2, \alpha}}^{2}:=\left(A_{\beta}^{\alpha} \omega_{k}, \omega_{k}\right)_{L_{\beta}^{2}}$; cf. Subsect. 3.2). Moreover, for all $Q \geq 1$ and $\alpha \in[0,1 / 2)$,

$$
\begin{gathered}
\sup _{\mu \in \mathcal{G}_{t}} \sup _{k \in \mathbb{Z}^{d}} \int_{\Omega}\left|\omega_{k}\right|_{W_{\beta}^{2, \alpha}}^{Q} d \mu(\omega)<\infty, \\
\sup _{\mu \in \mathcal{G}_{t}} \sup _{k \in \mathbb{Z}^{d}} \int_{\Omega}\left|V_{k}^{\prime}\left(\omega_{k}\right) \cdot \omega_{k}\right|^{Q} d \mu(\omega)<\infty, \\
\sup _{\mu \in \mathcal{G}_{t}} \sup _{k \in \mathbb{Z}^{d}} \int_{\Omega}\left|F_{k}^{V, W}(\omega)\right|_{L_{\beta}^{1}}^{Q} d \mu(\omega)<\infty .
\end{gathered}
$$

(ii) For the particular QLS models I, II from Section 2 (and also for the model III satisfying additionally Assumption $\left.\left(\boldsymbol{J}_{\mathbf{0}}\right)\right)$ (ii)) a priori estimates (7.67) always hold even for all $\mu \in \mathcal{G}_{t}:=\mathcal{G}_{(e) t}^{R} \supseteq \mathcal{G}_{(s) t}^{R}$.

Proof. (i) Let $p>d$ and let us take any $\mu \in \mathcal{G}_{t}=\mathcal{M}_{t}^{b}$ supported by $\mathcal{X}:=\Omega_{-p}^{R}$. For this $\mu$ we check the validity of the assumptions of Theorems $7.1^{\prime}$ and $7.3^{\prime}$. Recall (cf. Subsect. 3.2) that in this concrete setup we have the following single loop spaces over $S_{\beta}$ :

$$
X:=C_{\beta}, \quad H:=L_{\beta}^{2}, \quad B:=L_{\beta}^{R}, \quad B^{*}:=L_{\beta}^{R^{\prime}}
$$

and the spaces of scalar sequences over $\mathbb{Z}^{d}$ :

$$
E_{0}:=l^{2}\left(\mathbb{Z}^{d}\right), \quad E_{ \pm}:=l^{2}\left(\mathbb{Z}^{d} ; \gamma_{ \pm p}\right) .
$$

Actually, instead of the initial norm $\|\cdot\|_{\mathcal{L}_{-p}^{R}}:=\|\cdot\|_{-p, R}$ given by (3.12), we shall at once endow $\mathcal{B}:=\mathcal{L}_{-p}^{R}:=l^{2}\left(\mathbb{Z}^{d} \rightarrow L_{\beta}^{R} ; \gamma_{-p}\right)$ with a continuous system of mutually equivalent norms given by

$$
\|\omega\|_{-p, R, \sigma}:=\left[\sum_{k \in \mathbb{Z}^{d}}(1+\sigma|k|)^{-p}\left|\omega_{k}\right|_{L_{\beta}^{R}}^{2}\right]^{1 / 2}, \quad 0<\sigma \leq 1 .
$$

Note that each of the corresponding weight sequences $\gamma_{p, \sigma}:=\left\{(1+\sigma|k|)^{p}\right\}_{k \in \mathbb{Z}^{d}}$ satisfies the required properties (7.5)-(7.9). Besides, as can be easily verified, for any fast decreasing (i.e., satisfying Assumption $(\mathbf{J})$ with $M=2$ ) matrix $\mathcal{J}=\left\{\mathcal{J}_{k, j}\right\}_{k, j \in \mathbb{Z}^{d}}$ we have:

$$
\begin{aligned}
& \|\mathcal{J} \mid\|_{\sigma}:=\sup _{k \in \mathbb{Z}^{d}} \sum_{j \in \mathbb{Z}^{d}} \mathcal{J}_{k, j} \gamma_{p, \sigma, k-j}^{2 R}<\infty \\
& \lim _{\sigma \rightarrow+0}\left|\|\mathcal{J}\|_{\sigma}=\|\mathcal{J} \mid\|_{0}:=\sup _{k \in \mathbb{Z}^{d}} \sum_{j \in \mathbb{Z}^{d}} \mathcal{J}_{k, j} .\right.
\end{aligned}
$$

Then, as described in Subsect. 4.3, the partial logarithmic derivatives $b_{i}=b_{(k, n)}$ of each $\mu \in \mathcal{G}_{t}$ have the form (7.14) with the selfadjoint linear operator (cf. Subsect. 2.3.1)

$$
A=A^{*}:=A_{\beta}>0 \text { such that } \operatorname{Tr}_{H} A^{\alpha-1}<\infty, \forall \alpha<1 / 2,
$$


and with the smooth nonlinear components (cf. Lemma 4.6)

$$
\begin{gathered}
F_{k}^{0}:=F_{k}^{V} \in \bigcap_{n \in \mathbb{N}} C_{b, l o c}^{1}\left(X \rightarrow B^{*} ; \varphi_{n}\right), \\
G_{k}=F_{k}^{W} \in \bigcap_{i \in \mathbb{Z} \times \mathbb{N}} C_{b, l o c}^{1}\left(\mathcal{X} \rightarrow B^{*} ; h_{i}\right) .
\end{gathered}
$$

By Proposition 4.12 (i), the (IbP)-formula (7.16) holds for all $f \in C_{b}^{1}(\mathcal{X})$ satisfying the extra decay condition (7.17).

Furthermore, note that the required Assumptions $\left(\mathbb{F}_{0}\right)$ on the vector fields $F_{k}^{0}$ and resp. $\left(\mathbb{L}_{1-4}\right)$ on their coercivity functionals $L_{k}$ have already been obtained in (4.50) and (4.51). In our case

$$
\mathcal{K}_{3}:=K_{3}, \mathcal{K}_{4}:=K_{4}
$$

and the constant $\mathcal{K}_{4}>0$ could be taken arbitrarily small since so is $K_{4}$ in the initial Assumption $\left(\mathbf{V}_{\mathbf{i v}}\right)$ on the self-interaction potentials $V_{k}$. Analogously, the estimate (4.52) implies $\left(\mathbb{G}_{1}\right)$ and $\left(\mathbb{G}_{2}\right)$ with

$$
\mathcal{M}_{2}:=3 \cdot 2^{R}
$$

and the fast decreasing (cf. (J) and Lemma 3.4 (i)) matrix

$$
\mathcal{J}=\left\{\mathcal{J}_{k, j}\right\}_{k, j \in \mathbb{Z}^{d}}, \quad \mathcal{J}_{k, j}:=\left\{\begin{array}{cc}
\tilde{J}_{k, j}, & k \neq j \\
\|J\|_{0}, & k=j
\end{array}, \quad\|\mathcal{J}\|_{0} \leq 2\|J\|_{0} .\right.
$$

So, we can apply Theorems $7.1^{\prime}$ and $7.3^{\prime}$, provided for some fixed $0<\sigma \leq 1$,

$$
\Theta_{\sigma}^{\prime}:=3 K_{3} 2^{R}\|\mathcal{J}\|_{\sigma}<1
$$

But, by the continuity property (7.71), the relation (7.72) always holds for $\sigma \in\left[0, \sigma_{0}\right)$ as soon as

$$
\Theta_{0}^{\prime}:=6 K_{3} 2^{R}\|\mathbf{J}\|_{0}<1 .
$$

In turn, (7.73) as well as (3.55) (the latter is sufficient for the well-definedness of $\mu \in \mathcal{G}_{t}$, cf. Lemma 3.6) can be achieved by choosing $K_{3}>0$ small enough such that at least

$$
K_{3}^{-1}>3 R 2^{R}\|\mathbf{J}\|_{0}
$$

Taking into account the upper bounds (4.50) and (4.52) on $\left|F_{k}^{V, W}(\omega)\right|_{L_{\beta}^{1}}$, all this together gives us the following estimates for all $Q \geq 1$ and $0 \leq \alpha<1 / 2$ :

$$
\begin{gathered}
\sup _{k \in \mathbb{Z}^{d}} \int_{\Omega}\left|\omega_{k}\right|_{W_{\beta}^{2, \alpha}}^{Q} d \mu(\omega) \leq \mathcal{C}_{Q, \alpha}(p), \\
\sup _{k \in \mathbb{Z}^{d}} \int_{\Omega}\left|V_{k}^{\prime}\left(\omega_{k}\right) \omega_{k}\right|^{Q} d \mu(\omega) \leq \mathcal{C}_{Q}^{\prime}(p), \\
\sup _{k \in \mathbb{Z}^{d}} \int_{\Omega}\left|F_{k}^{V, W}(\omega)\right|_{L_{\beta}^{1}}^{Q} d \mu(\omega) \leq \mathcal{C}_{Q}^{\prime \prime}(p),
\end{gathered}
$$

which are uniform for all $\mu \in \mathcal{M}^{b}\left(\Omega_{-p}^{R}\right)$. Moreover, the first estimate in (7.75) obviously implies that any $\mu \in \mathcal{G}_{t}=\mathcal{M}_{t}^{b}$ is in fact supported on every $\Omega_{-p}^{R}$ as long as $p>d$. Hence (7.75) implies (7.67), and furthermore by the embedding theorem (3.4) that

$$
\sup _{\mu \in \mathcal{G}_{t}} \sup _{k \in \mathbb{Z}^{d}} \int_{\Omega}\left|\omega_{k}\right|_{L_{\beta}^{R^{\prime}}}^{Q} d \mu(\omega)<\infty, \quad \forall Q, R^{\prime} \geq 1 .
$$

(ii) The proof is analogous to that of (i), but with the following obvious modification. Since any $\mu \in \mathcal{G}_{t}:=\mathcal{G}_{(e) t}^{R}$ is supported by $\Omega_{-\delta}^{R}, \forall \delta>0$, in the concrete setup of Theorems $7.1^{\prime}$ and $7.3^{\prime}$ one should put $\mathcal{B}:=\mathcal{L}_{-\delta}^{R}:=l^{2}\left(\mathbb{Z}^{d} \rightarrow L_{\beta}^{R} ; \gamma_{-\delta}\right)$, 
$\mathcal{X}:=\Omega_{-\delta}^{R}:=\Omega \cap \mathcal{L}_{-\delta}^{R}$ and respectively $\gamma_{\delta}:=\{\exp \delta|k|\}_{k \in \mathbb{Z}^{d}}$ (cf. definitions (3.18)(3.20)). Then it is easy to check that all conditions of these theorems are satisfied with finite $\|\mathcal{J}\|:=\sup _{k \in \mathbb{Z}} \sum_{j \in \mathbb{Z}} \mathcal{J}_{k, j} \gamma_{\delta, k-j}^{R}<\infty$ and with arbitrarily small $\mathcal{K}_{3}, \mathcal{K}_{4}>0$.

Theorem 7.5 (cf. Hypothesis $\left(\mathbf{H}_{l o c}\right)$ : Uniform Estimates for Local Gibbs Specifications on Lebesgue and Sobolev Loops). Fix any boundary condition

$$
\xi \in \Omega_{t} \quad \text { with } \sup _{k \in \mathbb{Z}^{d}}\left|\xi_{k}\right|_{L_{\beta}^{R}}<\infty .
$$

Then under the assumptions of Theorem 7.5 the following uniform estimates on the local specifications $\pi_{\Lambda}(d \omega \mid \xi)$ hold for all $Q \geq 1$ and $0 \leq \alpha<1 / 2$ :

$$
\begin{gathered}
\sup _{\Lambda \Subset \mathbb{Z}^{d}} \sup _{k \in \Lambda} \int_{\Omega}\left|\omega_{k}\right|_{W_{\beta}^{2, \alpha}}^{Q} \pi_{\Lambda}(d \omega \mid \xi) \leq \mathcal{C}_{Q, \alpha}(\xi)<\infty, \\
\sup _{\Lambda \Subset \mathbb{Z}^{d}} \sup _{k \in \Lambda} \int_{\Omega}\left|V_{k}^{\prime}\left(\omega_{k}\right) \omega_{k}\right|_{L_{\beta}^{1}}^{Q} \pi_{\Lambda}(d \omega \mid \xi) \leq \mathcal{C}_{Q}^{\prime}(\xi)<\infty, \\
\sup _{\Lambda \Subset \mathbb{Z}^{d}} \sup _{k \in \Lambda} \int_{\Omega}\left|F_{k}^{V, W}(\omega)\right|_{L_{\beta}^{1}}^{Q} \pi_{\Lambda}(d \omega \mid \xi) \leq \mathcal{C}_{Q}^{\prime \prime}(\xi)<\infty .
\end{gathered}
$$

Proof. Actually, as follows from Theorems 6.2 and 6.5 applied to the finite volume Gibbs distributions $\nu_{\Lambda}\left(d \omega_{\Lambda} \mid \xi_{\Lambda^{c}}\right)$, for any $\Lambda \Subset \mathbb{Z}^{d}$ and $\xi \in \Omega_{t}$ the corresponding integrals in (7.67) are finite. We keep the concrete setting (7.68)-(7.70) already used in the proof of Theorem 7.5 for the loop lattice $\mathcal{X}=\Omega_{-p}^{R}$ with some $p>d$. In order to get the required bounds uniformly for all $\Lambda \Subset \mathbb{Z}^{d}$, let us go step by step through the scheme of the proof of the Theorems 7.1 and 7.3. Namely, setting $\varepsilon=\varepsilon^{\prime}=0$, let us apply the (IbP)-formula (4.57) along the directions $h_{i}, i=(k, n)$, when $k \in \Lambda$, to the test functions $f_{i}, g_{i}$ given by $(7.30),(7.31)$. Note that, in doing so, all the estimates $(7.32)-(7.42)$ are still valid for any $k \in \Lambda, \xi \in \mathcal{L}_{-p}^{R}$ and $\mu=\pi_{\Lambda}(d \omega \mid \xi)$. Since $\omega_{\Lambda^{c}}=\xi_{\Lambda^{c}}\left(\pi_{\Lambda}(d \omega \mid \xi)\right.$-a.e. $)$, taking in both sides of (7.43) and (7.50) the weighted sums with $\left(1+\sigma\left|k-k_{0}\right|\right)^{-p R}$ Over $k \in \Lambda$ and then adding to them in the obvious way the constants $\left(1+\sigma\left|k-k_{0}\right|\right)^{-p R}\left|\xi_{k}\right|_{L_{\beta}^{R}}^{R}$ when $k \notin \Lambda$, we conclude that

(i) $\quad \int_{\Omega}\|\mid \omega\|_{k, \sigma}^{R Q} \pi_{\Lambda}(d \omega \mid \xi) \leq \mathcal{C}_{Q, \sigma}\left(1+\|\xi\|_{k, \sigma}^{R Q}\right)$,

$$
\text { (ii) } \quad \int_{\Omega}\left(\sum_{j \in \mathbb{Z}^{d}} \mathcal{J}_{k, j}\left|\omega_{j}\right|_{L_{\beta}^{R}}^{R}\right)^{Q} \pi_{\Lambda}(d \omega \mid \xi) \leq \mathcal{C}_{Q, \sigma}^{\prime}\left(1+\|\xi\|_{k, \sigma}^{R Q}\right),
$$

where for shorthand we denote

$$
\|\xi\|_{k, \sigma}:=\left[\sum_{j \in \mathbb{Z}^{d}}(1+\sigma|j-k|)^{-p R}\left|\xi_{j}\right|_{L_{\beta}^{R}}^{R}\right]^{1 / R} \leq C_{\sigma}(1+|k|)^{p}\|\xi\|_{\mathcal{L}_{-p}^{R}}<\infty .
$$

Note that we have again used the same trick as in the proof of Theorem 7.5 by fixing a small enough value of $\sigma>0$ such that $\Xi_{0}, \Theta_{\delta}^{\prime}<1$. As soon as (7.79) is proved, from here on we can just repeat all the subsequent arguments from the proofs of Theorems $7.1,7.3$, resp. Theorems $7.1^{\prime}, 7.3^{\prime}$. In the final analysis, taking into account the upper bounds (4.50) and (4.52) on $\left|F_{k}^{V, W}(\omega)\right|_{L_{\beta}^{1}}$, we get that

$$
\begin{aligned}
& \int_{\Omega}\left|\omega_{k}\right|_{W_{\beta}^{2, \alpha}}^{Q} \pi_{\Lambda}(d \omega \mid \xi) \leq \mathcal{C}_{Q, \alpha, \sigma}\left(1+\mid\|\xi\|_{k, \sigma}^{2 R Q}\right)<\infty, \\
& \int_{\Omega}\left|V_{k}^{\prime}\left(\omega_{k_{0}}\right) \omega_{k_{0}}\right|_{L_{\beta}^{1}}^{Q} \pi_{\Lambda}(d \omega \mid \xi) \leq \mathcal{C}_{Q, \sigma}^{\prime \prime}\left(1+\|\xi\|_{k, \sigma}^{R Q}\right)<\infty, \\
& \int_{\Omega}\left|F_{k}^{V, W}(\omega)\right|_{L_{\beta}^{1}}^{R} \pi_{\Lambda}(d \omega \mid \xi) \leq \mathcal{C}_{Q, \sigma}^{\prime \prime \prime}\left(1+\|\xi\|_{k, \sigma}^{R Q}\right)<\infty .
\end{aligned}
$$

Moreover, it is important that all the constants $\mathcal{C}_{Q, \sigma}, \ldots, \mathcal{C}_{R, Q, \sigma}^{\prime \prime \prime}$ in the RHS in (7.79) and (7.81) are universal, i.e., do not depend on $k_{0}, \Lambda$ and $\xi$. Assuming now that the 
components of the loop sequence $\xi \in \mathcal{L}_{-p}^{R}$ are uniformly bounded, i.e.,

$$
\sup _{k \in \mathbb{Z}^{d}}\left|\xi_{k}\right|_{L_{\beta}^{R}}<\infty \text { and hence } \sup _{k \in \mathbb{Z}^{d}}\|\xi\|_{k, \sigma}^{Q}<\infty
$$

we get the desired estimates (7.78), which are also uniform in $k \in \Lambda$ and $\Lambda \Subset \mathbb{Z}^{d}$.

Remark 7.6. (i) Now we are in a position to complete the proof of Corollary 5.10 and hence of our Main Theorem III. Having use of (3.9), for the proof of statement (i) in Corollary 5.10 it suffices to show that $\|\xi\|_{\mathcal{L}_{-p}^{R}}<\infty$ implies

$$
\sup _{\Lambda \subseteq \mathbb{Z}^{d}} \sup _{k \in \Lambda}\left\{(1+|k|)^{-p R Q} \int_{\Omega}\left[\left|\omega_{k}\right|_{L_{\beta}^{2}}+\left|F_{k}^{V, W}(\omega)\right|_{L_{\beta}^{1}}\right]^{Q} \pi_{\Lambda}(d \omega \mid \xi)\right\}<\infty .
$$

But (7.82) readily follows from (7.79) (i), (7.80) and (7.81) (iii). On the other hand, modifying the concrete setup for the particular QLS models I-III as was described in the proof of Theorem 7.4 (ii) above, in much the same way one can show that $\|\xi\|_{\mathcal{L}_{-\delta}^{R}}<\infty$ implies

$$
\sup _{\Lambda \Subset \mathbb{Z}^{d}} \sup _{k \in \Lambda}\left\{e^{-\delta R Q|k|} \int_{\Omega}\left[\left|\omega_{k}\right|_{L_{\beta}^{2}}+\left|F_{k}^{V, W}(\omega)\right|_{L_{\beta}^{1}}\right]^{Q} \pi_{\Lambda}(d \omega \mid \xi)\right\}<\infty .
$$

In turn, as was pointed out in the proof of statement (ii) in Corollary 5.10, (7.83) and (3.9) yield for all $Q \geq 1, \delta^{\prime}>\delta R Q$ and $\alpha \in\left[0, \frac{1}{2}-\frac{1}{Q}\right)$,

$$
\sup _{\Lambda \Subset \mathbb{Z}^{d}}\left\{\sum_{k \in \Lambda} e^{-\delta^{\prime}|k|} \int_{\Omega}\left|\omega_{k}\right|_{C_{\beta}^{\alpha}}^{Q} \pi_{\Lambda}(d \omega \mid \xi)\right\}<\infty .
$$

Taking in (7.84) arbitrarily small $\delta>0$, resp. large $Q>1$, we get the desired estimate (2.20) in Main Theorem III.

(ii) Similarly one also can verify another Hypothesis $\left(\mathbf{H}_{p e r}\right)$ from Subsect. 5.2.2, which (according to Remark 5.11 (i), (ii)) provides us an alternative way to show the existence of $\mu \in \mathcal{G}_{t}$.

\section{Appendix: EuClideAn apPRoACh AND RECONSTRUCTION THEOREM}

According to the standard algebraic approach (cf. BrRo81]), equilibrium states in quantum statistical mechanics are defined as those normal states over a $C^{*}$-algebra $\mathcal{A}$ of quasi-local observables which satisfy the so-called KMS (Kubo-Martin-Schwinger) condition w.r.t. the one-parameter group $\alpha_{t}, t \in \mathbb{R}$, of time evolution automorphisms on $\mathcal{A}$. This approach is especially applicable for spin models with finite-dimensional physical Hilbert spaces corresponding to every single particle. But, unfortunately, the quantum lattice systems considered in this paper do not fit principally into the framework of such an algebraic approach, since there is no infinite volume dynamics for such models. Thus we use the following scheme for constructing their equilibrium states:

We begin with finite volumes $\Lambda \Subset \mathbb{Z}^{d}$ and local Hamiltonians

$$
\begin{aligned}
\mathbb{H}_{\Lambda}:= & -\frac{1}{2 \mathfrak{m}} \sum_{k \in \Lambda} \frac{d^{2}}{d x_{k}^{2}}+\frac{a^{2}}{2} \sum_{k \in \Lambda} x_{k}^{2} \\
& +\sum_{k \in \Lambda} V_{k}\left(x_{k}\right)+\sum_{M=2}^{N} \sum_{\left\{k_{1}, \ldots, k_{M}\right\} \subset \Lambda} W_{\left\{k_{1}, \ldots, k_{M}\right\}}\left(x_{k_{1}}, \ldots, x_{k_{M}}\right),
\end{aligned}
$$

which are well defined as selfadjoint operators in $\mathcal{H}_{\Lambda}:=L_{2}\left(\mathbb{R}^{\Lambda}, \times_{k \in \Lambda} d x_{k}\right)$. Then on the corresponding algebras of bounded linear operators $\mathcal{A}_{\Lambda}=\mathcal{L}\left(\mathcal{H}_{\Lambda}\right)$ we have the local 
automorphism groups $\alpha_{t, \Lambda}(A)=e^{i t H_{\Lambda}} A e^{-i t H_{\Lambda}}$ (the so-called Heisenberg dynamics), and the local Gibbs states at fixed inverse temperature $\beta>0$,

$$
G_{\beta, \Lambda}(A):=\operatorname{Tr}\left(A e^{-\beta H_{\Lambda}}\right) / \operatorname{Tr}\left(e^{-\beta H_{\Lambda}}\right), \quad A \in \mathcal{A}_{\Lambda} .
$$

Starting from these finite volume objects, one would like to construct the infinite volume automorphism group $\alpha_{t}(A)=\lim _{\Lambda \nearrow \mathbb{Z}^{d}} \alpha_{t, \Lambda}(A)$ and the limit Gibbs states

$$
G_{\beta, \Lambda}(A)=\lim _{\Lambda \nearrow \mathbb{Z}^{d}} G_{\beta, \Lambda}(A), \quad A \in \mathcal{A}_{l o c}:=\bigcup_{\Lambda \Subset \mathbb{Z}^{d}} \mathcal{A}_{\Lambda} .
$$

However, it should be strongly emphasized that for the systems under consideration it is impossible to control the thermodynamic limit (8.3) using the operator technique alone.

In order to overcome this principal difficulty, in AH-K75] an approach to the study of Gibbs states for quantum lattice systems, using Euclidean (rigorously defined) path space integrals, was initiated. Conceptually this approach is analogous to the well-known Euclidean strategy in quantum field theory (see, e.g., Sim74, Fro77, GJ81]). More precisely, the Euclidean method transforms the problem of giving a proper meaning to a quantum Gibbs state $G_{\beta}$ of the lattice system (8.1) at inverse temperature $\beta>0$ into the problem of constructing some (classical) Gibbs measure $\mu$ on the temperature loop lattice $\Omega=\left[C_{\beta}\right]^{\mathbb{Z}^{d}}$ (already defined by $(2.5)$ ). Due to this fact, various probabilistic techniques become available for the description of equilibrium properties of quantum infinite-particle systems. Here we only briefly outline these deep relations between quantum statistical mechanics and stochastic processes according to the initial paper AH-K75] (see also AKKR02 for the extended and up-to-date presentation):

For any finite set of multiplication operators $\left\{A_{0}, \ldots, A_{n}\right\} \subset L^{\infty}\left(\mathbb{R}^{\Lambda}\right)$, the spectral properties of the local Hamiltonians $\mathbb{H}_{\Lambda}$ enable us to define the so-called Matsubara (or temperature Euclidean Green) functions

$$
\begin{gathered}
\Gamma_{A_{0}, \ldots, A_{n}}^{\beta, \Lambda}\left(\tau_{0}, \ldots, \tau_{n}\right):=\operatorname{Tr}\left(e^{-\left(\beta-\left(\tau_{n}-\tau_{0}\right)\right) H_{\Lambda}} A_{n} \cdots e^{-\left(\tau_{1}-\tau_{0}\right) H_{\Lambda}} A_{0}\right) / \operatorname{Tr}\left(e^{-\beta H_{\Lambda}}\right), \\
0 \leq \tau_{0} \leq \cdots \leq \tau_{n} \leq \beta
\end{gathered}
$$

which have analytic continuations to the complex domain $0<\operatorname{Re} z_{0}<\cdots<\operatorname{Re} z_{n}<$ $\beta$ with the uniform bound $\left|\Gamma_{A_{0}, \ldots, A_{n}}^{\beta, \Lambda}\left(z_{0}, \ldots, z_{n}\right)\right| \leq\left\|A_{0}\right\|_{L^{\infty}} \cdots\left\|A_{n}\right\|_{L^{\infty}}$. Since on the boundary

$$
\Gamma_{A_{0}, \ldots, A_{n}}^{\beta, \Lambda}\left(-i \tau_{0}, \ldots,-i \tau_{n}\right)=G_{\beta, \Lambda}\left(\alpha_{\tau_{0}, \Lambda}\left(A_{0}\right) \cdots \alpha_{\tau_{n}, \Lambda}\left(A_{n}\right)\right), \quad \tau_{0}, \ldots, \tau_{n} \in \mathbb{R},
$$

and since (by the Høegh-Krohn theorem AH-K75]) the algebra spanned by the operators $\alpha_{\tau, \Lambda}(A)$ is a state detemining set, the Matsubara functions (8.4) fully determine the Gibbs state $G_{\beta, \Lambda}$.

A crucial observation of the Euclidean method is that Green functions (8.4) may be obtained as the moments of certain probability measures. More precisely, let $\gamma_{\beta}$ be a Gaussian measure on $C_{\beta}$ with correlation operator $A_{\beta}^{-1}$ (cf. (3.32)). Then by the Feynman-Kac formula,

$$
\Gamma_{A_{0}, \ldots, A_{n}}^{\beta, \Lambda}\left(\tau_{0}, \ldots, \tau_{n}\right)=\int_{C_{\beta}^{\Lambda}} A_{0}\left(\omega_{\Lambda}\left(\tau_{0}\right)\right) \cdots A_{n}\left(\omega_{\Lambda}\left(\tau_{n}\right)\right) d \mu_{\Lambda}\left(\omega_{\Lambda}\right),
$$

where the probability measure $\mu_{\Lambda}$ is defined by

$$
d \mu_{\Lambda}\left(\omega_{\Lambda}\right):=\frac{1}{Z_{\Lambda}} \exp \left\{-I_{\Lambda}\left(\omega_{\Lambda}\right)\right\} \times_{k \in \Lambda} d \gamma_{\beta}\left(\omega_{k}\right),
$$


with $\omega_{\Lambda}:=\left(\omega_{k}\right)_{k \in \Lambda} \in C_{\beta}^{\Lambda}$ and

$$
\begin{aligned}
I_{\Lambda}\left(\omega_{\Lambda}\right):= & \sum_{k \in \Lambda} \int_{S_{\beta}} V_{k}\left(\omega_{k_{1}}(\tau)\right) d \tau \\
& +\sum_{M=2}^{N} \sum_{\left\{k_{1}, \ldots, k_{M}\right\} \subset \Lambda} \int_{S_{\beta}} W_{\left\{k_{1}, \ldots, k_{M}\right\}}\left(\omega_{k_{1}}(\tau), \ldots, \omega_{k_{m}}(\tau)\right) d \tau .
\end{aligned}
$$

Suppose that a sequence $\left\{\mu_{\Lambda^{(K)}}\right\}_{K \in \mathbb{N}}$ converges (in the local weak sense) to some measure $\mu_{\infty}$ on $\Omega$. As follows from (8.6), this implies the existence of the limit temperature Green functions

$$
\begin{gathered}
\Gamma_{A_{0}, \ldots, A_{n}}^{\beta}\left(\tau_{0}, \ldots, \tau_{n}\right)=\lim _{\Lambda^{(K)} \nearrow \mathbb{Z}^{d}} \Gamma_{A_{0}, \ldots, A_{n}}^{\beta, A_{n}^{(K)}}\left(\tau_{0}, \ldots, \tau_{n}\right), \\
\forall A_{0}, \ldots, A_{n} \subset L_{\infty}\left(\mathbb{R}^{\Lambda}\right), \quad \Lambda^{(K)} \Subset \mathbb{Z}^{d},
\end{gathered}
$$

which satisfy the desired properties, such as analyticity and the so-called reflection positivity on a semicircle. As was analyzed on the axiomatic level in [AH-K75, KL81, BF02, there exists a correspondence (analogous to the Osterwalder-Schrader reconstruction theorem in Euclidean field theory [Sim74, GJ81]), between equilibrium states, temperature Green functions and stochastic processes. Namely, from $\Gamma_{A_{0}, \ldots, A_{n}}^{\beta}\left(\tau_{0}, \ldots, \tau_{n}\right)$ it is possible to construct (up to unitary equivalence) a Hilbert space $\mathcal{H}^{\Gamma}$ with a selfadjoint Hamiltonian $H$, a representation $\pi$ of the algebra of observables $\mathcal{A}$ on $\mathcal{H}^{\Gamma}$ with a cyclic vector $\psi_{\beta}$, and the von Neumann algebra $\mathcal{B} \subset L\left(\mathcal{H}^{\Gamma}\right)$ generated by $\pi(\mathcal{A})$, such that the state $G_{\beta}(B):=\left(\psi_{\beta}, B \psi_{\beta}\right)_{\mathcal{H}^{\Gamma}}$ is a KMS state on $\mathcal{B}$ w.r.t. the dynamics $\alpha_{t}(B):=e^{i t H} B e^{-i t H}$, $B \in \mathcal{B}$. On the other hand, due to their properties the functions $\Gamma_{A_{0}, \ldots, A_{n}}^{\beta}\left(\tau_{0}, \ldots, \tau_{n}\right)$ uniquely determine a measure $\mu$ on $\Omega$ such that

$$
E_{\mu}\left(A_{0}\left(\omega\left(\tau_{0}\right)\right) \cdots A_{n}\left(\omega\left(\tau_{n}\right)\right)\right)=\Gamma_{A_{0}, \ldots, A_{n}}^{\beta}\left(\tau_{0}, \ldots, \tau_{n}\right) .
$$

In fact, the measures $\mu_{\Lambda}$ in (8.7) correspond to the Gibbs distributions in the finite volumes $\Lambda$ with the empty boundary conditions of a lattice system on $\mathbb{Z}^{d}$ with the single spin space $C_{\beta}$. Due to this observation, it would appear reasonable to extend a class of limiting states to all Gibbs measures $\mu$ on $\Omega$ with the given Euclidean action functional $\left(I_{\Lambda}\right)_{\Lambda \Subset \mathbb{Z}^{d}}$. In general, the set of all Gibbs measures $\mathcal{G}=\{\mu\}$ is larger than the set of all accumulation points $\mu_{\infty}$ of $\left\{\mu_{\Lambda}\right\}$ when $\Lambda \nearrow \mathbb{Z}^{d}$. Nevertheless, it is important to note that from any such Gibbs measure $\mu$ we are able to reconstruct a state $G_{\beta}$ with the Euclidean Green functions (8.9). For the above reasons the measures $\mu \in \mathcal{G}_{\beta}$ will be called Euclidean Gibbs states (in the temperature loop space representation) for the quantum lattice system (3.1). Thus the Euclidean approach provides us not only a constuctive way to verify the existence of limiting Gibbs states in the traditional scheme of [BrRo81, but also enables us to extend substantially a class of states for the considered quantum lattice systems.

Nevertheless, despite a lot of papers dealing with the reconstruction theorem on an abstract level or in some concrete models (see e.g. [AH-K75, Fro77, GJO94, BF02, etc.), its consistent and mathematically rigorous implementation in the case of temperature Gibbs states for quantum lattice systems seems not to have been performed in the existing literature. We consider this as a very important open problem of high current interest, which we shall work on in the future. 


\section{ACKNOWLEDGMENT}

Financial support by the DFG through the Schwerpunktprogramm "Interacting Stochastic Systems of High Complexity" (Research Projects AL 214/17-2 and RO 1195/5) and by the Lise-Meitner Habilitation Programm is gratefully acknowledged.

\section{REFERENCES}

[AH-K75] S. Albeverio and R. Høegh-Krohn, Homogeneous random fields and quantum statistical mechanics, J. Funct. Anal. 19 (1975), 241-272. MR0378150 (51:14319)

[AH-K77] S. Albeverio and R. Høegh-Krohn, Dirichlet forms and diffusion processes on rigged Hilbert spaces, Z. Wahrsch. verw. Gebiete 40 (1977), 1-57. MR0455133 (56:13373)

[AKKR01] S. Albeverio, Yu. G. Kondratiev, Yu. V. Kozitzky, and M. Röckner, Uniqueness of Gibbs states of quantum lattices in small mass regime. Ann. Inst. H. Poincaré Probab. Statist. 37 (2001), 43-69. MR1815773 (2001m:82007)

[AKKR02] S. Albeverio, Yu. G. Kondratiev, Yu. V. Kozitzky, and M. Röckner, Euclidean Gibbs states of quantum lattice systems, Rev. Math. Phys. 14 (2002), 1335-1401. MR.1945807 (2004c:82017)

[AKKR03] S. Albeverio, Yu. G. Kondratiev, Yu. V. Kozitzky, and M. Röckner, Small mass implies uniqueness of Gibbs states of a quantum crystal, Comm. Math. Phys. 241 (2003), 69-90. MR2013752 (2004k:82010)

[AKMR98] S. Albeverio, Yu. G. Kondratiev, R. A. Minlos, and A. L. Rebenko, Small mass behaviour in quantum lattice models, J. Stat. Phys. 92 (1998), 1153-1172. MR1657797 (99j:82008)

[AKPR01a] S. Albeverio, Yu. G. Kondratiev, T. Pasurek (Tsikalenko), and M. Röckner, Gibbs states on loop lattices: Existence and a priori estimates, C. R. Acad. Sci. Paris Sér. I Math., 333 (2001), 1005-1009. MR.1872463(2002j:82011)

[AKPR01b] S. Albeverio, Yu. G. Kondratiev, T. Pasurek (Tsikalenko), and M. Röckner, Euclidean Gibbs states of quantum crystals, Moscow Math. J. 1 (2001), 1-7. MR.1877595 (2002k:82009)

[AKPR04] S. Albeverio, Yu. G. Kondratiev, T. Pasurek, and M. Röckner, Euclidean Gibbs measures on loop spaces: Existence and a priori estimates, Ann. Probab. 32 (2004), 153-190. MR.2040779 (2005b:82007)

[AKR97a] S. Albeverio, Yu. G. Kondratiev, and M. Röckner, Ergodicity of $L^{2}$-semigroups and extremality of Gibbs states, J. Funct. Anal. 144 (1997), 394-423. MR1432591 (98d:60187)

[AKR97b] S. Albeverio, Yu. G. Kondratiev, and M. Röckner, Ergodicity of the stochastic dynamics of quasi-invariant measures and applications to Gibbs states, J. Funct. Anal. 149 (1997), 415-469. MR1472365 (98j:60104)

[AKRe98] S. Albeverio, A. Yu. Kondratiev, and A. L. Rebenko, Peierls argument and long-range order behaviour in quantum lattice systems with unbounded spins, J. Stat. Phys. 92 (1998), 1153-1172. MR:1657872 (99k:82008)

[AKRT97a] S. Albeverio, Yu. G. Kondratiev, M. Röckner, and T.V. Tsikalenko, Uniqueness of Gibbs states for quantum lattice systems, Prob. Theory Rel. Fields 108 (1997), 193-218. MR 1452556 (99d:82009)

[AKRT97b] S. Albeverio, Yu. G. Kondratiev, M. Röckner, and T. V. Tsikalenko, Dobrushin's uniqueness for quantum lattice systems with nonlocal interaction. Comm. Math. Phys. 189 (1997), 621-630. MR 1480036 (98k:82013)

[AKRT99] S. Albeverio, Yu. G. Kondratiev, M. Röckner, and T.V. Tsikalenko, A priori estimates and existence of Gibbs measures: A simplified proof; C. R. Acad. Sci. Paris Sér. I Math. 328 (1999), 1049-1054. MR1696205 (2000d:60159)

[AKRT00] S. Albeverio, Yu. G. Kondratiev, M. Röckner, and T. V. Tsikalenko, A priori estimates for symmetrizing measures and their applications to Gibbs states, J. Funct. Anal. 171 (2000), 366-400. MR.1745630 (2001e:60205)

[AKRT01] S. Albeverio, Yu. G. Kondratiev, M. Röckner, and T. V. Tsikalenko, Glauber dynamics for quantum lattice systems, Rev. Math. Phys. 13 (2001), 51-124. MR1813614 (2002a:82084)

[Be85] D. Bell, A quasi-invariance theorem for measures on Banach spaces, Trans. Amer. Math. Soc. 290 (1985), 851-855. MR0792833 (86j:46043)

[Bo97] V.I. Bogachev, Differential measures and the Malliavin calculus, J. Math. Sci. 87 (1997), 3577-3731. MR1613197(99h:60111)

[BH-K82] J. Bellissard and R. Høegh- Krohn, Compactness and the maximal Gibbs states for random Gibbs fields on a lattice, Comm. Math. Phys. 84 (1982), 297-327. MR0667405|(83m:82002) 
[BaK91] V. S. Barbulyak and Yu. G. Kondratiev, Functional integrals and quantum lattice systems, Rep. Nat. Acad. Sci Ukraine, 8 (1991), 38-40; 9 (1991), 31-34; 10 (1991), 19-21. MR:1151528 (93b:82007a); 1149667 (93b:82007b) 1153563 (93b:82007c)

[BeK93] Yu. M. Berezansky and Yu. G. Kondratiev, Spectral Methods in Infinite Dimensional Analysis, Kluwer Academic Publishers, Dordrecht-Boston-London (1995). MR.1340626 (96d:46001a); 1340627 (96d:46001b)

[BF02] L. Birke and J. Fröhlich, KMS, etc., Reviews in Math. Phys. 14 (2002), 829-871. MR 1932668 (2004c:81155)

[BH00] T. Bodineau and B. Helffer, Correlations, spectral gap and log-Sobolev inequalities for unbounded spin systems, Differential Equations and Mathematical Physics (Birmingham, AL, 1999), 51-66, AMS/IP Stud. Adv. Math.16 (2000), AMS, Providence. MR1764741 (2001h:82011)

[BR01] V.I. Bogachev and M. Röckner, Elliptic equations for measures on infinite-dimensional spaces and applications, Prob. Theory Rel. Fields 120 (2001), 445-496. MR.1853480 (2002i:35193)

[BRW01] V.I. Bogachev, M. Röckner, and F.-Y. Wang, Elliptic equations for invariant measures on finite and infinite dimensional manifolds, J. Math. Pures Appl. 80 (2001), 177-221. MR.1815741 (2001k:58074)

[BrRo81] O. Bratteli and D. W. Robinson, Operator Algebras and Quantum Statistical Mechanics, I, II, Springer, Berlin-Heidelberg-New York (1981). MR0545651 (81a:46070); 0611508 (82k:82013)

[BY82] M. T. Barlow and M. Yor, Semimartingale inequalities via the Garsia-Rodemich-Rumsey lemma, and applications to local times, J. Funct. Anal. 49 (1982), 198-229. MR0680660 (84f:60073)

[Ca67] H. Cartan, Calcul Différentiel. Formes Différentielles, Hermann, Paris (1967). MR.0223194(36:6243)

[COPP78] M. Cassandro, E. Olivieri, A. Pellegrinotti, and E. Presutti, Existence and uniqueness of DLR measures for unbounded spin systems, Z. Wahrsch. verw. Gebiete 41 (1978), 313-334. MR 0471115 (57:10854)

[Dei85] K. Deimling, Nonlinear Functional Analysis, Springer, Berlin-Heidelberg-New York (1985) MR0787404 (86j:47001)

[Deu87] J.-D. Deuschel, Infinite-dimensional diffusion processes as Gibbs measures on $C[0,1]^{\mathbb{Z}^{d}}$. Probab. Th. Rel. Fields 76 (1987), 325-340. MR0912658 (89m:60252)

[Do70] R. L. Dobrushin, Prescribing a system of random variables by conditional distributions, Theory Prob. Appl. 15 (1970), 458-486. MR0298716 (45:7765)

[DLP79] W. Driesler, L. Landau, and J. F. Perez, Estimates of critical length and critical temperatures for classical and quantum lattice systems, J. Stat. Phys. 20 (1979), 123-162. MR.0523638 (80d:82035)

[DPZ96] G. Da Prato and J. Zabczyk, Ergodicity for Infinite-Dimensional Systems, Cambridge University Press (1996). MR1417491 (97k:60165)

[DaS88] Yu. L. Daletskii and G. A. Sokhadze, Absolute continuity of smooth measures, Funct. Anal. Appl. 22 (1988), 149-150. MR0947616 (89f:28036)

[DeuS89] J.-D. Deuschel and D.W. Strook, Large Deviations, Academic Press, London (1989). MR0997938 (90h:60026)

[Ed82] R. Edwards, Fourier Series. A Modern Introduction, Springer, Berlin-Heidelberg-New York (1982). MR0667519 (83k:42001)

[Fo82] H. Fölmer, A covariance estimate for Gibbs measures, J. Funct. Anal. 46 (1982), 387-395. MR.0661878 (84d:60142)

[Fri82] J. Fritz, Stationary measures of stochastic gradient dynamics, infinite lattice models, $Z$. Wahrsch. verw. Gebiete 59 (1982), 479-490. MR0656511(83j:60108)

[Fro77] J. Fröhlich, Schwinger functions and their generating functionals, II, Advances in Math. 33 (1977), 119-180. MR0436831 (55:9768b)

[Fu91] T. Funaki, The reversible measures of multi-dimensional Ginzburg-Landau type continuum model, Osaka J. Math. 28 (1991), 462-494. MR1144469 (93d:60105)

[FM99] W. G. Faris and R. A. Minlos, A quantum crystal with multidimensional anharmonic oscillators, J. Stat. Physics 94 (1999), 365-387. MR 1675357(2000e:82006)

[Ga02] O. Garet, Harmonic oscillators on an Hilbert space: A Gibbsian approach, Potent. Anal. 17 (2002), 65-88. MR 1906409 (2003e:60218)

[Ge88] H.-O. Georgii, Gibbs Measures and Phase Transitions, Studies in Mathematics, 9, Walter de Gruyter, Berlin-New York (1988). MR0956646 (89k:82010) 
[GJ81] J. Glimm and A. Jaffe, Quantum Physics. A Functional Integral Point of View, Springer, Berlin-Heidelberg-New York (1981). MR.0628000 (83c:81001)

[GJO94] R. Gielerak, L. Jakobczyk, and R. Olkiewicz, Reconstuction of Kubo-Martin-Schwinger structure from Euclidean Green functions, J. Math. Phys. 35 (1994), 3726-3744. MR 1279335 (95d:82008)

[GJS75] J. Glimm, A. Jaffe, and T. Spencer, Phase transitions for $\phi_{2}^{4}$ quantum fields, Comm. Math. Phys. 45 (1975), 203-216. MR0391797 (52:12616)

[GK90] S. A. Globa and Yu. G. Kondratiev, The construction of Gibbs states of quantum lattice systems, Selecta Math. Sov. 9 (1990), 297-307. MR0951149 (89h:60168)

[Ha01] Y. Hariya, A new approach to constructing Gibbs measures on $C\left(\mathbb{R} ; \mathbb{R}^{d}\right)$-an application for hard-wall Gibbs measures on $C(\mathbb{R}, \mathbb{R})$, Preprint (2001).

[HM00] B. Helffer and A. Martinez, Phase transition in the semiclassical regime, Rev. Math. Phys. 41 (2000), 1429-1450. MR.1809456 (2001k:81067)

[HS76] R. Holley and D. Stroock, $L_{2}$-theory for the stochastic Ising model, Z. Wahrsch. verw. Gebiete 35 (1976), 87-101. MR0410985 (53:14725)

[Iw85] K. Iwata, Reversible measures of a $P(\varphi)_{1}$-time evolution. Proc. Taniguchi Symp. PMMP (1985), 195-209. MR0933824 (90e:60092)

[J-LM85] G. Jona-Lasinio and P. K. Mitter, On the stochastic quantization of field theory, Comm. Math. Phys. 101 (1985), 409-436. MR.0815192 (87j:81246)

[Ki95] A.I. Kirillov, On the reconstruction of measures from their logarithmic derivatives, Izvestiya RAN: Ser. Mat. 59 (1995), 121-138. MR1328557(96e:46059)

[Ku82] S. Kusuoka, Dirichlet forms and diffusion processes on Banach space, J. Fac. Science Univ. Tokyo, Sec. 1A, 29 (1982), 79-95. MR0657873(83h:60082)

[KL81] A. Klein and L. Landau, Stochastic processes associated with KMS states, J. Funct. Anal. 42 (1981), 368-428. MR0626451 (84m:82033)

[KRZ96] Yu. G. Kondratiev, S. Roelly, and H. Zessin, Stochastic dynamics for an infinite system of random closed strings: A Gibbsian point of view, Stoch. Proc. Appl. 61 (1996), 223-248. MR.1386174 (97c:60145)

[Le01] M. Ledoux, Logarithmic Sobolev inequalities for unbounded spin systems revisited, Séminaire de Probabilités, XXXV, 167-194, Lecture Notes Math. 1755 (2001). MR.1837286 (2002g:82001)

[LP76] J. L. Lebowitz and E. Presutti, Statistical mechanics of systems of unbounded spins, Comm. Math. Phys. 50 (1976), 195-218. MR0446251(56:4579)

[MaMi95] V. A. Malyshev and R. A. Minlos, Linear Infinite-particle Operators, Translations of Mathematical Monographs 143, Amer. Math. Soc., Providence, RI, 1995. MR 1317349 (96d:82038)

[MRZ00] R. A. Minlos, S. Roelly, and H. Zessin, Gibbs states on space-time, Potent. Anal. 13 (2000), 367-408. MR1804179 (2002b:60103)

[MVZ00] R. A. Minlos, A. Verbeure, and V. A. Zagrebnov, A quantum crystal model in the lightmass limit: Gibbs states, Rev. Math. Phys. 12 (2000), 981-1032. MR 1782692(2001i:82012)

[OS99] H. Osada and H. Spohn, Gibbs measures relative to Brownian motion, Ann. Prob. 27 (1999), 1183-1207. MR:1733145 (2001f:82024)

[Pa67] K. R. Parthasarathy, Probability Measures on Metric Spaces. Academic Press, New YorkLondon (1967). MR.0226684 (37:2271)

[Pr76] C. Preston, Random Fields, Lect. Notes Math., 534, Springer, Berlin-Heidelberg-New York (1976). MR0448630 (56:6936)

[PK87] L. A. Pastur and B. A. Khoruzhenko, Phase transitions in quantum models of rotators and ferroelectrics, Theor. Math. Phys. 73 (1987), 1094-1104. MR0939799 (89c:82017)

[PaY94] Y. M. Park and H. J. Yoo, A characterization of Gibbs states of lattice boson systems, $J$. Stat. Phys. 75 (1994), 215-239. MR.1273059 (95c:82005)

[PaY95] Y. M. Park and H. J. Yoo, Uniqueness and clustering properties of Gibbs states for classical and quantum unbounded spin systems, J. Stat. Phys. 80 (1995), 223-271. MR1340559 $(96 \mathrm{j}: 82009)$

[Ro75] G. Royer, Unicité de certaines mesures quasi-invariantes sur $C(\mathbb{R})$, Ann. Scient. Éc. Norm. Sup., 4e série, 8 (1975), 455-478. MR0390161 (52:10987)

[Ro77] G. Royer, Étude des champs Euclidiens sur un réseau $\mathbb{Z}^{\gamma}$, J. Math. Pures et Appl. 56 (1977), 455-478. MR0468935 (57:8737)

[Rue69] D. Ruelle, Statistical Mechanics. Rigorous Results, Benjamin, New York-Amsterdam (1969). MR0289084 (44:6279) 
[RS75a] M. Reed and B. Simon, Methods of Modern Mathematical Physics. I. Functional Analysis, Academic Press, New York, London, 1972. MR0493419 (58:12429a)

[RS75b] M. Reed and B. Simon, Methods of Modern Mathematical Physics. II. Fourier Analysis, self-adjointness, Academic Press, New York, London, 1975. MR0493420 (58:12429b)

[Sh86] S. Shlosman, The method of reflection positivity in the mathematical theory of phase transitions of the first kind, Uspekhi Math. Nauk No. 3(249) (1986), 69-111. MR0854240 (88i:82074)

[Sim74] B. Simon, The $P(\varphi)_{2}$ Euclidean Field Theory, Princeton University Press, Princeton-New York (1974). MR0489552 (58:8968)

[Sim79] B. Simon, Functional Integration and Quantum Physics, Academic Press, New YorkLondon (1979). MR0544188 (84m:81066)

[Sin82] Ya. Sinai, Theory of Phase Transitions. Rigorous Results, Pergamon Press, Oxford (1982). MR.0691854 (84k:82002)

[Sk84] A. V. Skorohod, Integration on Hilbert space, Springer, Berlin-Heidelberg-New York (1984).

[Yo01] N. Yoshida, The equivalence of the log-Sobolev inequality and a mixing condition for unbounded spin systems on the lattice, Ann. Inst. H. Poincaré Prob. Statist. 37 (2001), 223-243. MR:1819124 (2001m:82025)

Institut für Angewandte Mathematik, Universität Bonn, D-53155 Bonn, Germany; BiBoS Research Centre, Bielefeld, Germany; and Cerfim, locarno, Switzerland

E-mail address: albeverio@uni-bonn.de

Fakultät für Mathematik and BiBos Research Centre, Bielefeld Universität, D-33615 Bielefeld, Germany; and Institute of Mathematics, NASU, Kiev, Ukraine

E-mail address: kondrat@mathematik.uni-bielefeld.de

BiBos Research Centre, Bielefeld Universität, D-33615 Bielefeld, Germany

E-mail address: pasurek@physik.uni-bielefeld.de

Fakultät für Mathematik and BiBos Research Centre, Bielefeld Universität, D-33615 Bielefeld, Germany

E-mail address: roeckner@mathematik.uni-bielefeld.de

English original provided by the authors 